Universidade de São Paulo

Instituto de Física

\title{
Estrutura eletrônica e dinâmicas de spins aplicadas a multicamadas metálicas e sistemas nanoestruturados
}

Ivan de Paula Miranda

Orientadora: Prof ${ }^{\mathrm{a}}$. Dr ${ }^{\mathrm{a}}$. Helena Maria Petrilli

Co-orientadora: Prof ${ }^{\mathrm{a}}$. Dr ${ }^{\mathrm{a}}$. Angela Burlamaqui Klautau

Tese de doutorado apresentada ao Instituto de Física como requisito parcial para a obtenção do título de Doutor em Ciências

\section{Banca Examinadora:}

Prof $^{\mathrm{a}}$. Dr ${ }^{\mathrm{a}}$. Helena Maria Petrilli (orientadora) - IFUSP

Prof. Dr. Luis Gregório Godoy de Vasconcellos Dias da Silva - IFUSP

Prof. Dr. Roberto Bechara Muniz - UFF

Prof. Dr. Julio Criginski Cesar - LNLS

Prof. Dr. Rubem Luis Sommer - CBPF

São Paulo 
University of São Paulo

Physics Institute

\title{
Electronic structure and spin dynamics applied to metallic multilayers and nanostructured systems
}

\author{
Ivan de Paula Miranda
}

Supervisor: Prof. Dr. Helena Maria Petrilli

Co-supervisor: Prof. Dr. Angela Burlamaqui Klautau

Thesis submitted to the Physics Institute of the University of São Paulo in partial fulfillment of the requirements for the degree of Doctor of Science

\section{Examining Committee:}

Prof. Dr. Helena Maria Petrilli (supervisor) - IFUSP

Prof. Dr. Luis Gregório Godoy de Vasconcellos Dias da Silva - IFUSP

Prof. Dr. Roberto Bechara Muniz - UFF

Prof. Dr. Julio Criginski Cesar - LNLS

Prof. Dr. Rubem Luis Sommer - CBPF

São Paulo 
FICHA CATALOGRÁFICA

Preparada pelo Serviço de Biblioteca e Informação

do Instituto de Física da Universidade de São Paulo

Miranda, Ivan de Paula

Estrutura eletrônica e dinâmicas de spins aplicadas a multicamadas metálicas e sistemas nanoestruturados. São Paulo, 2021.

Tese (Doutorado) - Universidade de São Paulo. Instituto de Física. Depto. de Física dos Materiais e Mecânica.

Orientador: Profa. Dra ${ }^{a}$. Helena Maria Petrilli

Área de Concentração: Física da Matéria Condensada

Unitermos: 1. Física do Estado Sólido; 2. Magnetismo; 3. Fenômenos Magnéticos; 4. Física Estatística.

USP/IF/SBI-005/2021 


$$
\begin{array}{r}
\text { À meus pais, Nina e José, } \\
\text { e à minha esposa, Juliana, } \\
\text { com carinho. }
\end{array}
$$




\section{Agradecimentos}

Terminar uma tese de doutorado no contexto de uma pandemia não foi fácil. $O$ presente trabalho é fruto de uma ação conjunta, e de uma extensa rede de apoio. Sendo assim, agradeço:

à Deus pela paz nos momentos difíceis, e por guiar minha vida;

à Prof ${ }^{\mathrm{a}}$. Dr ${ }^{\mathrm{a}}$. Helena Maria Petrilli, que teve a coragem de me aceitar como aluno mesmo sabendo que eu estive fora da vida acadêmica por alguns anos, os quais me dediquei às atividades bancárias e educacionais. Como orientadora dedicada, pacientemente me ensinou como ser um pesquisador, e me mostrou que a Ciência não progride apenas com números, mas também com palavras;

à Prof ${ }^{\mathrm{a}}$. Dr ${ }^{\mathrm{a}}$. Ângela Burlamaqui Klautau Crispino, pela co-orientação, por sempre me receber da melhor forma em Belém, e por acreditar em minha carreira científica. Com certeza nossas discussões foram fundamentais para a construção do meu conhecimento;

ao Prof. Dr. Olle Eriksson, por me aceitar como aluno durante 6 meses na Universidade de Uppsala e confiar em meu trabalho, e aos Drs. Anders Bergman, Danny Thonig, Attila Szilva, e Manuel Pereiro, pelas discussões científicas, e por fazerem de minha estadia na Suécia a melhor possível;

à meus pais, Nina de Paula Brito de Miranda e José Augusto Brito de Miranda, por me ensinarem que com honestidade e companheirismo chega-se longe na vida. Serei eternamente grato pelo pão de cada dia, e pelo seu amor e dedicação imensos, em todos os momentos;

à minha esposa, Juliana Santos Oliveira, pelo carinho, companheirismo, e conselhos verdadeiros. Também por me incentivar na carreira científica, dando sempre o melhor de si por mim. Este trabalho não considero meu, mas nosso;

aos meus avós maternos, Maria Fomin de Paula e Sebastião de Paula, por me ensinarem 
a trabalhar duro para conseguir realizar os meus sonhos. Também por me educarem com sacrifício durante meus anos escolares;

à família da minha esposa, Raimundo Oliveira, Maria de Fátima, e Ricardo Oliveira, por cederem um espaço em sua casa para que eu e a Juliana pudéssemos morar, e por todo o carinho e dedicação que sempre apresentaram para conosco;

aos meus avós paternos (in memorian), Maria Conceição Bastos Brito de Miranda e José Benício Nunes de Miranda, pela generosidade e companhia às minhas estadias em Campinas ;

à minha família de Campinas/Cosmópolis/Rio de Janeiro, Therezinha, Cristina, Chico, Renata, Afonso, Raoni e Carlos Alberto, pelos agradáveis natais e momentos juntos;

aos colegas (eventuais ou não) do Departamento de Física dos Materiais, que muito me ajudaram nesta jornada e por tornarem o ambiente de trabalho agradável: Pamela Costa, Bruno Ipaves, Eduardo Suárez, Ana Valencia, Bruna Shinohara, Arles Rebaza, Filipe Dalmatti, Marcos Brown, Ricardo Igarashi, Michel Lacerda, Samuel Silva e Luiz Eleno;

aos amigos do Angström Laboratory, Rodrigo Carvalho, Ageo Andrade, José Silva, Josene Toldo, e Yocefu Hattori, pelas conversas, pelas risadas, e por me darem todo o suporte físico e emocional durante a minha estadia em Uppsala;

aos funcionários do Departamento, em especial à Sandra, à Rosana, e ao Luiz por toda ajuda administrativa e conversas de corredor;

à Prof ${ }^{\mathrm{a}}$. Dr ${ }^{\mathrm{a}}$. Lucy Vitória Credidio Assali, por toda a orientação em perovskitas, e por me transmitir uma outra visão sobre a Física do estado sólido e cálculos de estrutura eletrônica;

aos demais professores do Departamento de Física dos Materiais, pelo conhecimento transmitido, pela motivação, e por ajudarem também a tornar o ambiente de trabalho agradável;

aos amigos do IFUSP, Uiran, Stevan, Jullian, Evandro, Thiago, Nimay, Hugo, Lari, Daniel, Ari, Rodrigo, Guilherme, Felipe, Suigh, Vini, Ribolli, Marcelo e outros, por fazerem dos meus anos de graduação os mais divertidos e loucos que alguém poderia desejar;

aos meus amigos do Albert Sabin, Richard, Tiago Bardella, Rheda, Ivan, Caio, Guilherme Lopes, Flávio, Guilherme Vargas, Guilherme Nascimento, Renato, André Ericsson, André Filipe, Portella, Tiago Bardella, Toni, Kirita, Gabriel Arruda, e muitos outros pelos 
inesquecíveis anos de colégio;

aos professores do Albert Sabin, que muito me ensinaram, e formaram a base de tudo o que sei hoje;

ao antigo Laboratório de Computação Científica Avançada da USP (LCCA-USP), ao Centro Nacional de Processamento de Alto Desempenho em São Paulo (CENAPADUnicamp), ao National Supercomputer Centre (NSC-Suécia), e ao Laboratório Nacional de Computação Científica (LNCC-MCTI), e seus respectivos funcionários, pela disponibilização dos recursos computacionais necessários para a execução deste trabalho; e, finalmente,

às agências de fomento à pesquisa, FAPESP, CNPq, e ao INEO pelo indispensável apoio financeiro (dentro e fora do Brasil) e pela concessão de auxílios para conferências. O presente trabalho foi realizado com apoio da Coordenação de Aperfeiçoamento de Pessoal de Nível Superior - Brasil (CAPES) - Código de Financiamento 001. 
"The beauty of a living thing is not the atoms that go into it, but the way those atoms are put together"

\section{CARL SAGAN}




\section{Resumo}

Neste trabalho, apresentamos o estudo teórico de vários materiais magnéticos nanoestruturados. Para estes sistemas investigamos a estrutura eletrônica e propriedades magnéticas, via cálculos baseados na Teoria do Funcional da Densidade (DFT), e/ou dinâmica da magnetização no contexto da construção atomística de spins.

Para o magnetismo de superfície (ou sistemas de baixa dimensionalidade), apresentamos cálculos ab-initio da estrutura eletrônica de bicamadas de $\mathrm{Pd} / \mathrm{Fe}$ e $\mathrm{Pd} / \mathrm{Co}$ sobre um substrato de $\operatorname{Ir}(111)$, com o foco na investigação dos mecanismos microscópicos responsáveis pela formação de configurações magnéticas de spin não-colineares. Utilizando os momentos magnéticos e parâmetros de troca isotrópicos e anisotrópicos (DzyaloshinskiiMoriya) obtidos via cálculos de primeiros princípios, realizamos simulações da dinâmica de spins baseadas na aproximação atomística (modelo de Landau-Lifshitz-Gilbert) nestes e em outros sistemas de interesse, visando analisar os efeitos de temperatura e/ou da aplicação de campo magnético nas texturas magnéticas destes sistemas e comparar com dados experimentais, se existentes.

Adicionalmente, implementamos o cálculo do damping $(\alpha)$ no método de primeiros princípios no espaço-real Real Space-Linear $\mathbf{M}$ uffin $\mathbf{T}$ in $\mathbf{O}$ rbital-Atomic Sphere Approximation (RS-LMTO-ASA). As potencialidades desta metodologia foram exploradas no estudo da anisotropia de damping na superfície de $\mathrm{Fe}_{50} \mathrm{Co}_{50}(100)$ e da influência causada por defeitos $4 d$ e $5 d$ na multicamada $\mathrm{Pd} / \mathrm{Fe} / \mathrm{Ir}(111)$, de forma a ter uma descrição mais precisa deste parâmetro em sistemas com quebra de simetria como aglomerados embebidos em superfícies.

Esta é a primeira vez que o cálculo ab-initio de espaço-real para a interação de Dzyaloshinskii-Moriya é aplicado em conjunto com a dinâmica atomística de spins a fim de se estudar skyrmions a temperatura e campo finitos. Além disso, é inédita a investigação do damping em sistemas heterogêneos e/ou aglomerados contendo impurezas. 


\section{Abstract}

Here we present theoretical investigation of several nanostructured magnetic materials. The electronic structure and magnetic properties are obtained via first-principles calculations based on the Density Functional Theory (DFT), and/or magnetization dynamics in the context of the atomistic spins framework.

For the surface magnetism (or low dimension systems), we present ab-initio calculations of the electronic structure of the $\mathrm{Pd} / \mathrm{Fe}$ and $\mathrm{Pd} / \mathrm{Co}$ bilayers deposited on $\operatorname{Ir}(111)$, focusing on the investigation of the microscopic mechanisms which are responsible for the formation of noncollinear magnetic configurations. Using the magnetic moments, and isotropic and anisotropic (Dzyaloshinskii-Moriya) parameters obtained via first-principles calculations, we performed simulations of the spin dynamics based on the atomistic approximation (Landau-Lifshitz-Gilbert model) in these and others systems of interest, aiming to analyze the effects of temperature and/or the application of an external magnetic field in the magnetic textures of these systems and compare with experimental data, if available.

Additionally, we implemented the damping $(\alpha)$ calculation in the first-principles realspace method Real Space-Linear $\mathbf{M}$ uffin $\mathbf{T}$ in $\mathbf{O}$ rbital-Atomic $\mathbf{S}$ phere Approximation (RS-LMTO-ASA). The potential of this methodology was explored in the study of the damping anisotropy in the $\mathrm{Fe}_{50} \mathrm{CO}_{50}(100)$ surface and in the influence of $4 d$ and $5 d$ defects on the $\mathrm{Pd} / \mathrm{Fe} / \mathrm{Ir}(111)$ multilayer, to have a more precise description of this parameter in systems with lack of inversion symmetry, such as clusters embedded in surfaces.

This is the first time that a real-space ab initio calculation for the DzyaloshinskiiMoriya interaction is used together with atomistic spin dynamics simulations to study skyrmions subjected to finite temperature and external magnetic fields. Furthermore, the investigation of the damping parameter in heterogeneous systems and clusters containing impurities is new on literature. 


\section{Conteúdo}

1 Introdução 1

2 Tópicos em magnetismo $\quad 7$

2.1 A interação de troca . . . . . . . . . . . . . . . . 7

2.2 Ordenamentos magnéticos: colinear e não-colinear . . . . . . . . . . . . . 9

2.3 Magnetismo geometricamente frustrado . . . . . . . . . . . . . . . 11

2.4 Acoplamento spin-órbita . . . . . . . . . . . . . . . . . . . . 12

2.5 Interação de Dzyaloshinskii-Moriya $(\mathrm{DM}) \ldots \ldots \ldots$. . . . . . . . . 14

2.6 Skyrmions . . . . . . . . . . . . . . . . . . . . . 15

2.6.1 Carga topológica $q \ldots \ldots \ldots \ldots$

2.7 Energia de anisotropia magnética (MAE) . . . . . . . . . . . . 24

3 O método RS-LMTO-ASA $\quad 27$

3.1 Teoria do Funcional da Densidade e métodos aproximativos . . . . . . . . . 27

3.1 .1 Esquema de Kohn-Sham . . . . . . . . . . . . . . . . . . 29

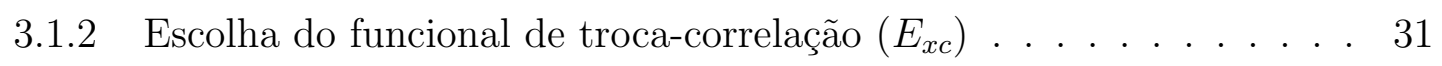

3.2 Formalismo LMTO-ASA no espaço direto (RS-LMTO-ASA) . . . . . . . . 32

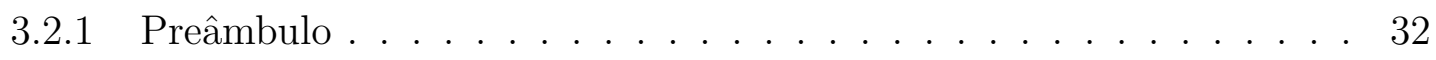


3.2 .2 Implementação do RS-LMTO-ASA . . . . . . . . . . . . . . . . . . 34

3.2.3 Processo autoconsistente para sistemas bidimensionais (superfícies) no RS-LMTO-ASA . . . . . . . . . . . . . . . . . . . . 39

3.2.4 Processo autoconsistente para defeitos em superfícies no RS-LMTO-

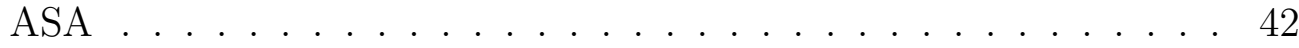

3.2.5 Cálculo da interação de troca $\left(\mathcal{J}_{i j}\right) \ldots \ldots$. . . . . . . . . . . 44

3.2.6 Cálculo da interação de Dzyaloshinskii-Moriya . . . . . . . . . . . . 47

4 Dinâmica atomística de spins $\quad 52$

4.1 Equação de movimento . . . . . . . . . . . . . . . . . . . . . . 52

4.2 Efeitos de temperatura: dinâmica de Langevin . . . . . . . . . . . . . . 55

4.3 Método de Monte Carlo (clássico) . . . . . . . . . . . . . . . . 57

$5 \quad$ Skyrmions em filmes ultrafinos $\quad 59$

$5.1 \mathrm{Pd} / \mathrm{Fe} / \mathrm{Ir}(111) \ldots \ldots \ldots \ldots \ldots \ldots \ldots$

5.1 .1 Preâmbulo . . . . . . . . . . . . . . . . . 59

5.1.2 Propriedades eletrônicas da bicamada de $\mathrm{Pd} / \mathrm{Fe} / \operatorname{Ir}(111) \ldots . . .60$

$5.2 \mathrm{Pd} / \mathrm{Co} / \operatorname{Ir}(111) \ldots \ldots \ldots \ldots \ldots \ldots \ldots$

5.3 Comparação entre as bicamadas . . . . . . . . . . . . . . . . . . 96

5.4 Influência de defeitos no sistema $\mathrm{Pd} / \mathrm{Fe} / \mathrm{Ir}(111) \ldots \ldots$. . . . . . . 106

5.5 Skyrmions em outras multicamadas . . . . . . . . . . . . . . . . 108

6 Damping no espaço-real $\quad 114$

6.1 Formalismo . . . . . . . . . . . . . . . . . . . . . . . 114

6.1.1 Damping de Gilbert para materiais com diferentes tipos atômicos 120

6.2 Primeiros testes em sistemas bulk . . . . . . . . . . . . . . . 122 


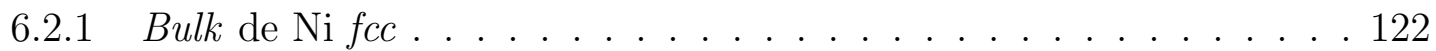

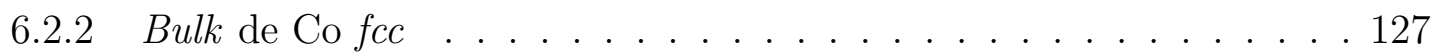

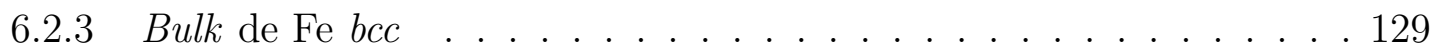

6.3 Ligas de $\mathrm{Fe}_{1-x} \mathrm{Co}_{x}$ e $\mathrm{Ni}_{1-x} \mathrm{Fe}_{x} \ldots \ldots \ldots \ldots$. . . . . . . . . . . . . . 132

6.4 Tabela de comparação . . . . . . . . . . . . . . . . . . 135

6.5 Cálculo do broadening $\left(\Lambda_{R S}\right) \ldots \ldots \ldots$. . . . . . . . . . . . . . . . . . . .

6.6 Damping nas superfícies de $\mathrm{Pd} / \mathrm{Fe}$ e $\mathrm{Pd} / \mathrm{Co}$ em $\operatorname{Ir}(111) \ldots . . . .140$

6.6.1 Influência de impurezas de $\operatorname{Ir}(5 d)$ e $\mathrm{Pd}(4 d)$ no damping local . . . 141

6.7 Anisotropia de damping: o caso da superfície de $\mathrm{Fe}_{50} \mathrm{Co}_{50}(100) \ldots . . . .145$

\section{Conclusões}

A Interação de DM como efeito do spin-órbita

B Formalismo LMTO-ASA

B.1 Orbitais lineares do tipo muffin-tin (LMTO) e o formalismo LMTO-ASA no espaço $\mathbf{k}$ 183

B.1.1 Matrizes Hamiltoniana $(\mathcal{H})$ e de overlap $(\mathbf{O}) \ldots . . . . . . . .184$

B.1.2 Potenciais do tipo muffin-tin . . . . . . . . . . . . . . . 185

B.1.3 Aproximação da esfera atômica (ASA) . . . . . . . . . . . . . . . 189

B.1.4 Obtenção da base canônica $\left\{\chi_{R L}^{0}\right\} \ldots$. . . . . . . . . . . 191

B.1.5 Formalismo LMTO-ASA na base genérica . . . . . . . . . . . . 198

B.1.6 Base tight-binding $(\mathrm{TB}) \ldots \ldots . \ldots . . \ldots . . \ldots 201$

B.1.7 Base ortogonal . . . . . . . . . . . . . . . . . 203

B.1.8 Representação ortogonal da matriz $\mathcal{H}$ em termos de parâmetros da base TB . . . . . . . . . . . . . . . . . . . . 205 
B.2 O Método de Recorrência _ . . . . . . . . . . . . . . . 207

C Formalismo LKAG perturbado

D Arquivo de entrada damping.in

F UppASD com defeitos: implementação

F.1 Repetição de impurezas no espaço . . . . . . . . . . . . . . . . . 221

F.2 Impureza isolada . . . . . . . . . . . . . . . . . . . . . 223 


\section{Capítulo 1}

\section{Introdução}

Conhecido desde a antiguidade, o fenômeno do magnetismo sempre acompanhou a evolução do conhecimento e o avanço tecnológico da humanidade. Desde Tales de Mileto ( 624 - 546 a.C.), filósofo e matemático grego que atribuiu a existência de uma alma, e, portanto, vida aos materiais magnéticos [1], até as pesquisas mais recentes, que tratam o magnetismo como uma propriedade naturalmente emergente da configuração eletrônica e das interações de origem quântica entre os elétrons, é certo que a investigação de materiais magnéticos nos levou a importantes progressos ao longo da história, apresentando aplicações em diversas áreas, tais como: em medicina [2-5], na catalização de reações químicas [6], na extração de petróleo [7], em nanotecnologia [8-11], e outras. No entanto, não há dúvidas que um dos principais interesses na aplicação do magnetismo, sobretudo a partir da segunda metade do século XX, seja em dispositivos de gravação e leitura digital, que desempenharam um papel decisivo no desenvolvimento dos computadores em geral, além de aparelhos de áudio e vídeo [12].

De modo simultâneo a esses avanços na utilização do magnetismo em dispositivos de gravação e leitura, ocorreu o aprimoramento das técnicas experimentais [13], capazes de manipular e caracterizar fisicamente estruturas inclusive de extensão atômica. Dentre essas técnicas, destacam-se: $(i)$ o microscópio de força atômica (AFM - Atomic Force Microscope), baseado na detecção de forças da ordem de $10^{-18} \mathrm{~N}$ [14]; (ii) o microscópio de corrente de tunelamento (STM - Scanning Tunneling $\mathbf{M}$ icroscope) [15], e sua variação com polarização de spin (SP-STM) [13]; (iii) a técnica de X-Ray Magnetic Circular Dichroism (XMCD) [16], que permite a obtenção experimental dos valores de momento magnético de spin e orbital, além da energia de anisotropia magnética do sistema, a partir das regras de soma [17]; entre outros. Ademais, não podemos deixar de mencionar a inerente evolução assistida pelas próprias técnicas de fabricação dos dispositivos, o que 
permitiu dobrar (ou até triplicar) sua capacidade de gravação a cada $\sim 2$ anos [18].

O princípio fundamental do armazenamento e da leitura de dados é que a informação pode ser decomposta em unidades binárias (ou bits), sendo que a criação ou aniquilação dos bits em um meio magnético depende, direta ou indiretamente, da utilização de materiais que apresentam magnetização espontânea - ou, no mínimo, controlável. A existência desta característica em sólidos regulares, ligas, ou estruturas de menor dimensão (mono/multicamadas e pequenos aglomerados atômicos) está relacionada, necessariamente, à presença de efeitos microscópicos na amostra. Por exemplo, o ferromagnetismo de sistemas metálicos, em especial os que incluem metais de transição, está sujeito a uma dualidade aparente no comportamento dos elétrons da camada $d$ [19]: por um lado, a natureza itinerante do estado fundamental desses elétrons pode ser descrita por teoria de bandas [20]; por outro, em temperaturas finitas, esses mesmos portadores de carga apresentam propriedades que inicialmente eram atribuídas apenas a sistemas com momento magnético localizado. O mesmo se aplica aos dispositivos eletrônicos tradicionais, nos quais toda a informação está baseada no transporte da carga eletrônica, e sua interpretação no processamento conjunto de inúmeras portas lógicas.

A relativamente recente possibilidade de utilizar o spin do elétron, ao invés de apenas a sua carga, como unidade fundamental de informação foi essencial ao desenvolvimento de novos dispositivos que combinam a microeletrônica usual com efeitos relacionados ao spin e seus graus de liberdade, ramo denominado spintrônica [21]. Um dos desafios atualmente existentes na spintrônica é o desenvolvimento de dispositivos de memória baseados no controle, por meio de correntes elétricas, da configuração magnética do material em escala nanométrica [22]. Essa solução mostra-se particularmente interessante por integrar vantagens como baixo custo, densa capacidade de gravação, não-volatilidade [23] e alta performance - características que não são observadas conjuntamente em dispositivos eletrônicos existentes no mercado, como os discos rígidos (HDDs) convencionais. Sendo assim, observou-se nos últimos anos um intenso esforço de pesquisa de estruturas magnéticas capazes de serem controladas no material sob essas condições, tais como: defeitos topológicos em forma de vórtice conhecidos como skyrmions [22, 24-27], ou paredes de domínio [28-31]. Ambas as propostas estão relacionadas ao fenômeno conhecido como magnetismo não-colinear.

Dentre as manifestações do magnetismo em escala nanométrica, um dos mais extraordinários é o surgimento de texturas não-colineares de spin, isto é, um ordenamento altamente correlacionado no qual os momentos de spin não se alinham a uma única direção no espaço - mas apresentam rotações ao longo de toda ou parte da extensão do material. 
A natureza desse tipo de configuração magnética em sistemas de estado sólido é bastante rica, e diferentes expressões do magnetismo não-colinear foram observadas graças ao esforço conjunto de pesquisas teóricas e experimentais: além das supracitadas, podemos ainda mencionar outras texturas complexas de spin [32-36], efeitos de paridade [37, 38], e demais configurações circulares em nanoestruturas magnéticas [39, 40].

Particularmente no contexto dos skyrmions, ao qual será direcionado nosso foco neste trabalho, a existência de propriedades topológicas confere a essas estruturas uma enorme robustez morfológica, o que os transforma em candidatos promissores para sua atuação como portadores de unidades de informação magnética [41]. Embora experimentalmente sua observação tenha ocorrido primeiro em materiais bulk, como nos cristais de MnSi (de estrutura cúbica do tipo B20) [42], as pesquisas atuais têm se concentrado majoritariamente na investigação de sistemas bidimensionais, isto é, de materiais organizados em mono ou multicamadas. Uma das principais razões para isso é que as recentes propostas de aplicação de skyrmions estão baseadas em dispositivos de gravação do tipo racetrack [27, 43-45] (veja a Figura 1.1, abaixo), que intrinsecamente seguem a simetria bidimensional.

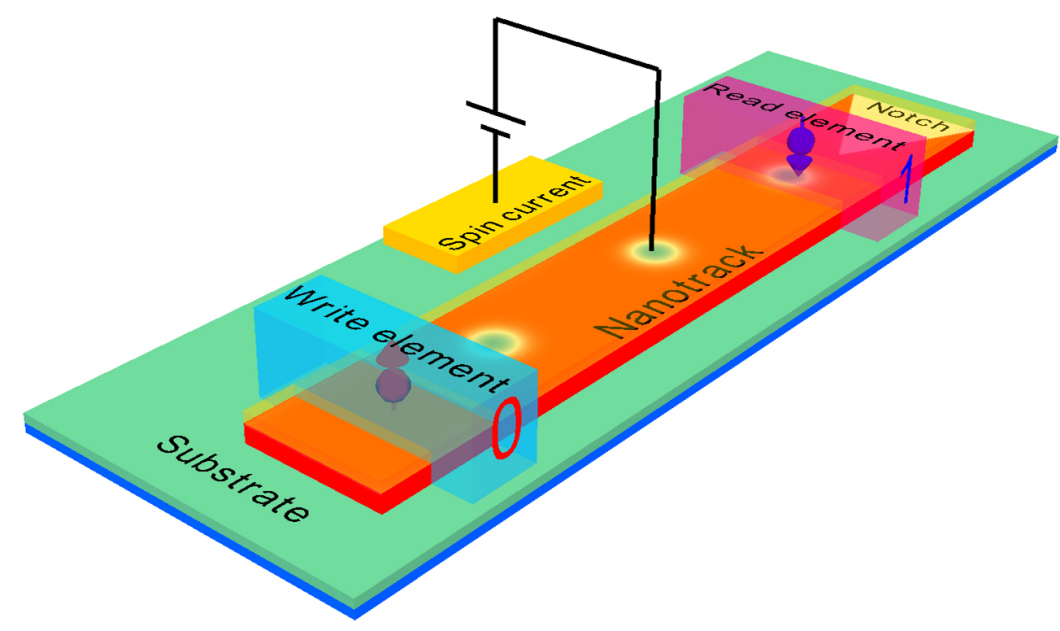

Figura 1.1: Proposta de um dispositivo de gravação e leitura magnética do tipo racetrack baseado em skyrmions. Aqui, a presença de cada defeito topológico está associada ao bit "1" de informação. Os skyrmions são movimentados pela ação de correntes elétricas aplicadas ao material, e interpretados pelo cabeçote de leitura do dispositivo (posicionado ao fim da faixa de deslocamento). Figura reproduzida da referência [27].

As primeiras evidências de skyrmions nessa classe de sistemas ocorreram em 2011, quando Heinze et al. [46] reportaram a obtenção de uma rede dessas estruturas topológicas como estado fundamental de uma monocamada de Fe depositada em $\operatorname{Ir}(111)$. A explicação dada pelos autores para a estabilidade dessa configuração magnética foi 
atribuída à atuação conjunta das interações de troca (no modelo de Heisenberg [47]), Dzyaloshinskii-Moriya (DM) [48, 49], e four-spin. O segundo termo (DM) se refere a uma interação quiral que emerge naturalmente em um ambiente com quebra de simetria de inversão espacial, e que é influenciada pelos efeitos do acoplamento spin-órbita [50]. Já o terceiro termo (four-spin) se refere a um acoplamento associado ao efeito do hopping eletrônico em 4 sítios adjacentes [51], e que confere uma simetria quadrada aos skyrmions emergentes no material.

Dois anos mais tarde, Romming et al. [52] propuseram incluir uma camada de $\mathrm{Pd}$ acima do Fe no sistema de Fe/Ir(111), justamente para possibilitar o controle do surgimento de skyrmions por meio da aplicação de um campo magnético externo, e por argumentarem que, devido à elevada polarizabilidade do $\mathrm{Pd}$, a bicamada de $\mathrm{Pd} / \mathrm{Fe}$ se comportaria como uma única entidade magnética. Esse trabalho foi especialmente importante, pois nele se demonstrou, de forma pioneira na literatura, que a criação/aniquilação de um único skyrmion é viável experimentalmente por meio da introdução de correntes locais ao sistema através de um microscópio de corrente de tunelamento com polarização de spin (SP-STM [13]). Assim, a bicamada de Pd/Fe/Ir(111) configura-se como um dos materiais conhecidos que apresentam uma fase de skyrmions, no qual o estado fundamental é caracterizado por uma configuração magnética em espiral (de spin) [53, 54]. Quando esse sistema é sujeito à ação de um campo magnético perpendicular à superfície (da ordem de $\sim 1-2 \mathrm{~T}[52,55]$, à baixas temperaturas), observa-se a emergência de uma rede de skyrmions.

Assim, um importante problema atualmente em aberto é o entendimento das origens microscópicas e das condições favoráveis para o surgimento do magnetismo não-colinear em um dado material, em especial para aquele que apresenta uma fase de skyrmions - e, devido às suas propriedades magnéticas, a bicamada de $\mathrm{Pd} / \mathrm{Fe} / \mathrm{Ir}(111)$ desponta como uma proposta bastante adequada para este fim. As explicações teóricas mais comuns presentes na literatura para a estabilização do ordenamento não-colinear em sistemas bidimensionais envolvem frustrações magnéticas [56], a existência de interações não-desprezíveis do tipo Dzyaloshinskii-Moriya na interface [57], e um correto balanço entre o preenchimento das bandas e o exchange splitting do material [58] - ou ainda, uma ação conjunta dessas três características, já que, em certo nível, pode-se dizer que estão inter-relacionadas. Embora já existam alguns trabalhos publicados que investiguem o comportamento magnético da bicamada de $\mathrm{Pd} / \mathrm{Fe} / \mathrm{Ir}(111)[52-55,59,60]$, pouco ainda foi explorado sobre as origens microscópicas do ordenamento não-colinear nesse sistema.

A fim de preencher essa lacuna, neste trabalho nós realizamos, em um primeiro mo- 
mento, a comparação entre as bicamadas de $\mathrm{Pd} / \mathrm{Fe} / \operatorname{Ir}(111)$ e $\mathrm{Pd} / \mathrm{Co} / \mathrm{Ir}(111)$. Esta última apresenta um estado fundamental ferromagnético [61], e suas propriedades magnéticas locais não foram completamente investigadas até o presente momento, via métodos teóricos. Além destas duas bicamadas, aproveitamos o método desenvolvido aqui para o estudo de outras multicamadas metálicas, que podem também apresentar propriedades interessantes (e com potencial aplicação tecnológica) e trazer novos insights sobre a origem dos skyrmions em superfícies magnéticas.

Por outro lado, um segundo foco deste trabalho será a investigação da dinâmica de magnetização nestes sistemas. Nesse sentido, o estado-da-arte no estudo teórico de sistemas magnéticos baseia-se na realização de cálculos ab-initio de estrutura eletrônica, e a posterior utilização de métodos para a investigação da dinâmica de spins [62]. Como é sabido na literatura, um modelo fenomenológico importante para este tipo de análise é a equação de Landau-Lifshitz-Gilbert [63]. No contexto deste modelo, destaca-se a consideração de um amortecimento (damping) no movimento dos spins, que tem atraído bastante atenção de uma parte da comunidade de física do estado sólido devido à sua recente possibilidade de cálculo via primeiros princípios [64-68]. O entendimento do damping é fundamental para futuras aplicações que procuram explorar o grau de liberdade do spin eletrônico, uma vez que este parâmetro pode afetar tanto a energia necessária quanto a velocidade que um dispositivo pode operar. Sendo assim, no presente trabalho de pesquisa, além da implementação do damping no espaço-real, pretendemos estudá-lo em sistemas variados, de maneira a trazer novas compreensões sobre os mecanismos que o conduzem nestas situações.

A presente tese está organizada da seguinte maneira. No Capítulo 2, resumimos os tópicos em nanomagnetismo mais relevantes para o entendimento dos resultados obtidos. Já no Capítulo 3, descrevemos o método autoconsistente de primeiros princípios no espaço direto utilizado na realização de nossos cálculos, o RS-LMTO-ASA, além da forma como são incluídas as interações de troca e de DM nesse contexto. Por sua vez, o formalismo do qual esse método é derivado, o LMTO-ASA, é desenvolvido em detalhes no Apêndice B, em termos das bases canônica, genérica, ortogonal e mais localizada (ou tight-binding). A parte de metodologia é encerrada com o capítulo 4, no qual descreve-se a dinâmica atomística de spins, método utilizado para se obter um diagrama preliminar de fases magnéticas para a bicamada de $\mathrm{Pd} / \mathrm{Fe} / \operatorname{Ir}(111)$. Nos Capítulos 5 e 6 , apresentamos nossos resultados. Usando o método RS-LMTO-ASA discutimos o papel de cada tipo de interação (de troca e DM) na configuração dos distintos estados fundamentais, além de sua natureza de longo alcance, e as principais diferenças que favorecem a existência de estados não-colineares estáveis e metaestáveis no $\mathrm{Pd} / \mathrm{Fe} / \operatorname{Ir}(111)$, obtendo também 
via Uppsala Atomistic Spin Dynamics (UppASD) um diagrama de fases com base no cálculo da carga topológica associada a cada par de campo magnético e temperatura $(B, T)$. Além disso, fazemos uma análise da influência de um defeito de Ir na multicamada $\mathrm{Pd} / \mathrm{Fe} / \mathrm{Ir}(111)$, bem como do comportamento magnético de outras multicamadas na presença de um campo magnético externo e temperaturas finitas. O amortecimento (damping), sua implementação, testes, e aplicação em sistemas e situações inéditas também são apresentados neste Capítulo. Finalmente, no Capítulo 7, apresentamos e discutimos as conclusões obtidas.

Além das pesquisas apresentadas na presente tese, foram publicados também trabalhos referentes a outros temas como resultado do período de doutoramento, que podem ser encontrados nas referências [69-71]. 


\section{Capítulo 2}

\section{Tópicos em magnetismo}

A interação entre spins eletrônicos é a base não só para o estabelecimento do ordenamento magnético em um material como também para toda teoria quântica do magnetismo. Ao que se sabe, os fenômenos de origem magnética são governados pelos elétrons existentes em um sistema, e a grande variedade de efeitos observada experimentalmente ou prevista pela teoria se deve ao enorme conjunto de arranjos espaciais e elementos que podem ser escolhidos ao se formar um sistema.

Portanto, a análise de sistemas que vão desde corpos extensos (bulk) até nanoestruturas pode passar por pelo menos um modelo de interação eletrônica apresentado neste capítulo. Em vista da investigação desenvolvida no presente trabalho, o entendimento dos tipos de ordenamento, da origem da anisotropia magnética, e dos modelos teóricos que são capazes de explicar determinados arranjos de spins, será útil para a discussão dos resultados obtidos.

\subsection{A interação de troca}

A ideia de uma interação entre átomos vizinhos $i$ e $j$ com spins eletrônicos $\mathbf{s}_{i}$ e $\mathbf{s}_{j}$ denominada interação de troca - surge naturalmente do tratamento quântico de elétrons como partículas indistinguíveis, e está relacionada tanto à superposição da função de onda $\psi$ desses elétrons quanto à necessidade de tornar $\psi$ uma função antissimétrica, de acordo com o princípio de Pauli.

Tomando a situação com dois elétrons apenas, a dependência da energia total do sistema com a orientação relativa dos spins pode ser introduzida por um termo escalar 
$\mathbf{s}_{1} \cdot \mathbf{s}_{2}$ na Hamiltoniana, multiplicada por constante de proporcionalidade $-\mathcal{J}_{12}$ (parâmetro de troca):

$$
\mathcal{H}_{\text {exc }}=-\mathcal{J}_{12}\left(\mathbf{s}_{1} \cdot \mathbf{s}_{2}\right)
$$

Em um sólido, a presença de átomos individuais e mais complexos que o hidrogênio favorece uma aproximação conceitual simples, mas poderosa: a definição da Hamiltoniana de Heisenberg (Eq. 2.1) a partir dos spins atômicos totais, isto é, a substituição $\mathbf{s} \rightarrow \mathbf{S}$ [72]. Obviamente, ao se aplicar essa ideia em um material finito, o termo de energia de troca deve conter todas as interações entre os átomos, tomadas dois a dois. Assim, aproveitando a notação estabelecida anteriormente:

$$
\mathcal{H}_{e x c}=-\sum_{i \neq j} \mathcal{J}_{i j}\left(\mathbf{S}_{i} \cdot \mathbf{S}_{j}\right)
$$

A consideração dos spins atômicos no modelo deixa-o mais próximo do esboço elaborado por Weiss [73], no qual o campo molecular origina-se das interações entre os momentos magnéticos dos átomos. Embora a substituição $\mathbf{s} \rightarrow \mathbf{S}$ facilite de forma considerável a resolução da Hamiltoniana da Eq. 2.1, ainda o elevado número de sítios não equivalentes no sólido cristalino (isto é, com interações assimétricas entre spins) impede a demonstração de uma solução única e geral deste modelo.

A Eq. 2.2 é amplamente utilizada na descrição de sistemas magnéticos, e em geral trata de forma satisfatória estruturas com momentos magnéticos localizados, para os quais a interação com vizinhos mais distantes pode ser desprezada $\left(\mathcal{J}_{i j} \sim 0\right.$, se $d_{i j}$ é muito grande) - em especial, os materiais isolantes [74]. É importante ressaltar que os momentos magnéticos dos metais de transição (MT), quando formam ligações metálicas, são basicamente diferentes dos momentos em átomos livres, e, por apresentarem estados eletrônicos não-localizados, neste caso seus momentos magnéticos não são dados por um número inteiro de elétrons com spins desemparelhados [75]. De fato, a elaboração de critérios rigorosos para a validade do modelo de Heisenberg para um dado sistema é uma tarefa extremamente difícil, e usualmente supõe-se que este modelo seja aplicável no mapeamento teórico das propriedades magnéticas. No caso de sistemas ferromagnéticos com elétrons itinerantes, entretanto, o alcance das interações de troca podem ser significativamente maiores, e a aproximação $\mathcal{J}_{i j} \sim 0$ não deve ser empregada considerando-se uma extensão maior de vizinhos [76]. Para um dado material, seguindo essa definição, o estado ferromagnético (FM) entre dois spins nos sítios $i$ e $j$ ocorre quando $\mathcal{J}_{i j}>0$, e o estado antiferromagnético (AFM) ocorre quando $\mathcal{J}_{i j}<0$. 
Neste trabalho, o comportamento magnético das bicamadas de $\mathrm{Pd} / \mathrm{Fe} / \mathrm{Ir}(111)$ e de $\mathrm{Pd} / \mathrm{Co} / \mathrm{Ir}(111)$ é discutido à luz do modelo clássico de Heisenberg, implementado na forma sugerida por Liechtenstein et al. [77], que dá origem à abordagem incluída no método RS-LMTO-ASA (descrito de forma detalhada na seção 3.2.5).

\subsection{Ordenamentos magnéticos: colinear e não-colinear}

A associação de dois ou mais constituintes atômicos induz o overlap entre as funções de onda, dando origem ao chamado magnetismo coletivo. Por outro lado, nos sólidos metálicos (como também em outros materiais) a aproximação de um grande número de átomos produz, por atuação do princípio de exclusão de Pauli, níveis de energia muito próximos uns dos outros, o que leva à formação das bandas de energia. Portanto, o comportamento magnético dos materiais em estado sólido não se torna mais tão previsível como o de um átomo livre, e está sujeito à tendência de hopping dos elétrons entre sítios vizinhos (hibridização de estados eletrônicos), à geometria local, e às competições entre as interações de troca, responsáveis pela magnetização espontânea de certas regiões da amostra. Assim, por exemplo, ainda que localmente os átomos possuam um momento magnético considerável, se a interação $\mathcal{J}_{i j}$ é suficientemente fraca, não haverá um ordenamento global de longo alcance no material. Em contrapartida, interações de Heisenberg intensas entre os constituintes pode gerar uma vasta gama de configurações magnéticas em nível microscópico.

(a)

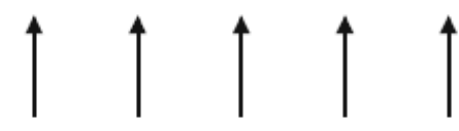

(c)

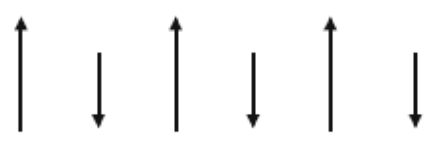

(b)

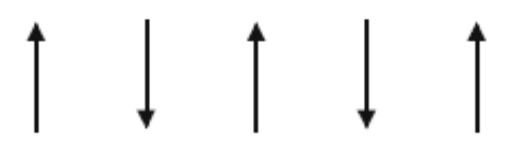

(d)
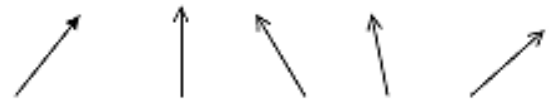

Figura 2.1: Tipos de ordenamento magnético: (a) ferromagnético (FM); $(b)$ antiferromagnético (AFM); $(c)$ ferrimagnético (FI); e $(d)$ não-colinear. As setas representam as direções dos momentos de spin atômicos.

Assim, denomina-se magnetismo colinear a situação na qual os momentos magnéticos 
de spin dos átomos se alinham ao longo de um único eixo global de quantização. A orientação paralela entre os spins de sítios vizinhos caracteriza o estado ferromagnético (FM), como pode ser visto na Fig. 2.1(a). O caso antiferromagnético (AFM), por sua vez, ocorre quando os momentos de spin alinham-se antiparalelamente (Fig. 2.1(b)), de forma que a magnetização total para grandes extensões no material seja nula. Por fim, existe também o ordenamento ferrimagnético (FI), no qual os momentos são antiparalelos, embora apresentem magnitudes distintas (Fig. 2.1(c)).

O critério de Stoner [78] é um modelo que qualitativamente estabelece que o material será ferromagnético se [19]

$$
\operatorname{In}\left(E_{F}\right)>1
$$

onde $I$ é o chamado parâmetro de Stoner (ou energia de exchange), uma quantidade que varia lentamente ao longo da tabela periódica, e $n\left(E_{F}\right)$ é a densidade de estados nãomagnética no nível de Fermi. O modelo de Stoner, do qual deriva a Eq. 2.3, é baseado na estatística de Fermi e trata as interações elétron-elétron na aproximação de campo médio. Levando em conta a susceptibilidade magnética dada por $\chi=\frac{2 \mu_{B}^{2} n\left(E_{F}\right)}{\left[1-\operatorname{In}\left(E_{F}\right)\right]}[19,79]$, e a magnetização induzida por um campo externo uniforme $\mathbf{H}$, com $\mathbf{M}=\chi \mathbf{H}$, nos materiais em que se observa o fenômeno de magnetização espontânea, a tendência ao alinhamento dos momentos de spin em qualquer situação é expressa pelo limite $\lim _{|\mathbf{H}| \rightarrow 0}|\mathbf{M}|>0$, ou seja, válido para um campo $\mathbf{H}$ arbitrariamente pequeno. Isto só ocorre se $\chi \rightarrow \infty$, ou $\operatorname{In}\left(E_{F}\right) \rightarrow$ 1, o que delimita o critério para o surgimento do estado FM espontâneo na teoria de Stoner. Modelos análogos podem ser apresentados para justificar o ordenamento AFM. De fato, outras teorias podem ser empregadas para descrever a preferência do sistema em questão por um ordenamento particular, como é o caso da competição entre os mecanismos de kinetic exchange [80] e double exchange [81, 82], que favorecem, respectivamente, os acoplamentos AFM e FM entre os sítios.

Caso não haja um eixo global de magnetização, então a configuração de interesse é classificada como não-colinear (Fig. 2.1(d)). Embora seja razoável atribuir as causas dos ordenamentos FM e AFM a teorias bem estabelecidas, a origem do comportamento nãocolinear ainda não é completamente compreendida. Uma das possibilidades, explorada no presente trabalho, é a presença de interações antissimétricas de Dzyaloshinskii-Moriya (DM) [48, 49] não-desprezíveis, mediadas pelo acoplamento spin-órbita (acoplamento spin-órbita). O termo de DM favorece a existência de estruturas magnéticas espacialmente rotacionadas, tendo como referência uma direção de rotação específica governada pelo vetor $\mathbf{D}_{i j}$ (seção 2.5). Em sistemas cristalinos com simetria de inversão, entre eles os materiais bulk mais simples, as interações antissimétricas são nulas ou relativamente in- 
significantes, uma vez que o espalhamento dos elétrons no processo de hopping, conduzido pelo acoplamento spin-órbita, é isotrópico [50]. Por outro lado, em sistemas cuja simetria de inversão é quebrada, a sequência de espalhamento de elétrons com spin polarizado entre os sítios, por exemplo, $i \rightarrow j \rightarrow i$ e $j \rightarrow i \rightarrow j$ torna-se não-comutativa, aumentando a relevância do termo de DM em relação à interação de troca proposta pelo modelo de Heisenberg (Eq. 2.2) - e podendo, inclusive, conduzir à estrutura final não-colinear dos momentos de spin. Outra possível causa da configuração não-colinear em sistemas de estado sólido é a presença de frustrações magnéticas com origem nas interações de troca, e que serão explicadas na próxima seção.

\subsection{Magnetismo geometricamente frustrado}

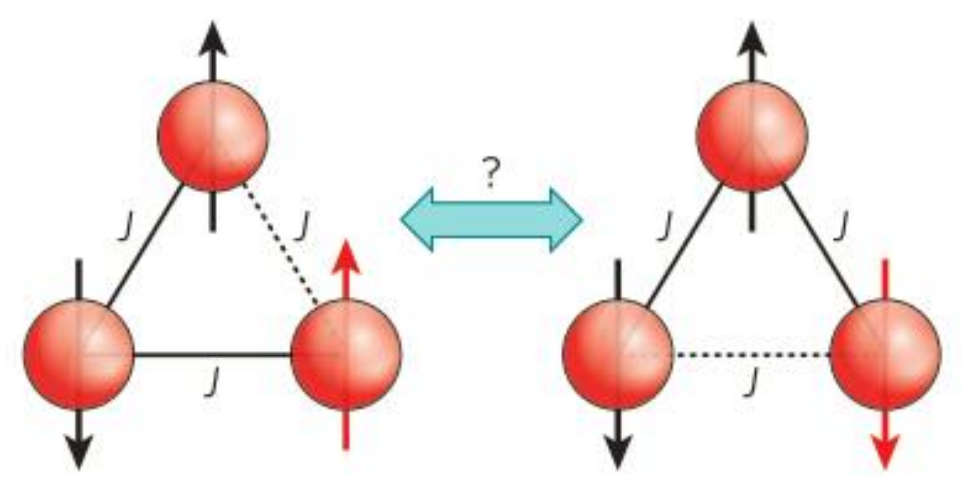

Figura 2.2: Representação esquemática de uma rede triangular AFM frustrada [83]. Se a interação de troca $J$ entre os momentos de spin é sempre negativa, não há alinhamento colinear trivial que resolva a Hamiltoniana de Heisenberg e leve à configuração de menor energia.

As explicações teóricas mais comuns para o ordenamento não-colinear espontâneo envolvem os efeitos de frustração geométrica dos spins (ou, simplesmente, frustração magnética) [37, 39, 58]. Este fenômeno está associado ao fato de, em determinadas situações, não existirem soluções para o Hamiltoniano de Heisenberg que satisfaçam a preferência pelo ordenamento colinear AFM entre todos os vizinhos mais próximos de um dado sítio - particularmente devido à configuração espacial do sistema. O caso clássico é o de três átomos dispostos nos vértices de um triângulo equilátero (Fig. 2.2). Se $\{1,2,3\}$ são os índices que representam as posições destes átomos, e $\mathcal{J}_{i j}<0$ para $\forall i, j \in\{1,2,3\}$, então os ângulos colineares triviais entre os momentos de $\operatorname{spin}\left(\vartheta_{i j}=\pi\right.$ e $\left.\vartheta_{i j}=0\right)$ não são os que minimizam a Hamiltoniana da Eq. 2.2, na sua representação clássica. Esta afirmação é demonstrada como se segue. Para fins de simplificação, suponha que $\mathcal{J}_{i j} \equiv J$, 
onde $J$ é uma constante negativa expressa em unidades de energia, e que os spins $X Y$ $\mathbf{S}_{i, j}$ dispostos nesta configuração, tenham magnitude $S$ fazendo um ângulo $\theta_{i}$ com o eixo $x$. Logo, a energia $E$ associada a este sistema será dada por [84]:

$$
\begin{aligned}
& E=J \sum_{i \neq j} \mathbf{S}_{i} \cdot \mathbf{S}_{j}=J\left(\mathbf{S}_{1} \cdot \mathbf{S}_{2}+\mathbf{S}_{2} \cdot \mathbf{S}_{3}+\mathbf{S}_{3} \cdot \mathbf{S}_{1}\right) \\
= & J S^{2}\left[\cos \left(\theta_{1}-\theta_{2}\right)+\cos \left(\theta_{2}-\theta_{3}\right)+\cos \left(\theta_{3}-\theta_{1}\right)\right]
\end{aligned}
$$

Portanto, a fim de encontrar os valores de $\theta_{1}, \theta_{2}$, e $\theta_{3}$ que minimizam a função $E \equiv$ $E\left(\theta_{1}, \theta_{2}, \theta_{3}\right)$ (Eq. 2.4), basta tomar sua primeira derivada em relação a cada ângulo $\theta_{i}$ $(i=1,2,3)$ e forçá-la ser zero, isto é:

$$
\left\{\begin{array}{l}
\frac{\partial E}{\partial \theta_{1}}=-J S^{2}\left[\operatorname{sen}\left(\theta_{1}-\theta_{2}\right)-\operatorname{sen}\left(\theta_{3}-\theta_{1}\right)\right]=0 \\
\frac{\partial E}{\partial \theta_{2}}=-J S^{2}\left[\operatorname{sen}\left(\theta_{2}-\theta_{3}\right)-\operatorname{sen}\left(\theta_{1}-\theta_{2}\right)\right]=0 \\
\frac{\partial E}{\partial \theta_{3}}=-J S^{2}\left[\operatorname{sen}\left(\theta_{3}-\theta_{1}\right)-\operatorname{sen}\left(\theta_{2}-\theta_{3}\right)\right]=0
\end{array}\right.
$$

As três relações da Eq. 2.5 são simultaneamente satisfeitas se $\left(\theta_{1}-\theta_{2}\right)=\left(\theta_{2}-\theta_{3}\right)=$ $\left(\theta_{3}-\theta_{1}\right) \Rightarrow \theta_{i}=\frac{2 \pi}{3}$ (ou $\left.\theta_{i}=\frac{4 \pi}{3}\right)$, levando, obviamente, a uma configuração magnética na qual não há um eixo global de magnetização, ou não-colinear. Outra maneira de demonstrar a não-colinearidade entre os momentos de spin dispostos em uma rede triangular equilátera é reescrever $J \sum_{i \neq j} \mathbf{S}_{i} \cdot \mathbf{S}_{j}=-\frac{3}{2} J S^{2}+\frac{J}{2}\left(\mathbf{S}_{1}+\mathbf{S}_{2}+\mathbf{S}_{3}\right)^{2}$, que é minimizada se $\left(\mathbf{S}_{1}+\mathbf{S}_{2}+\mathbf{S}_{3}\right)^{2} \equiv 0$ - produzindo a estrutura com os momentos de spin inclinados $120^{\circ}$ em relação ao eixo $x$ [84]. A frustração magnética também pode ser encontrada em outros sistemas simples, como em um rede linear com condições periódicas de contorno.

\subsection{Acoplamento spin-órbita}

Sob um ponto de vista clássico, o acoplamento spin-órbita pode ser entendido a partir da referência de um elétron em movimento com velocidade $\mathbf{v}$, sujeito a um campo elétrico externo E. Relativamente ao núcleo, o movimento da carga eletrônica leva à indução de um campo magnético $\mathbf{B} \sim \frac{1}{c^{2}} \mathbf{v} \times \mathbf{E}$, que se acopla ao momento intrínseco ao elétron, na forma $\mathbf{m} \cdot \mathbf{B}$, e produz um termo adicional na sua Hamiltoniana. Embora associado ao cenário clássico mencionado, o acoplamento spin-órbita é um efeito relativístico, e surge do tratamento da equação de Dirac. Assim, convém apresentar de maneira introdutória a derivação deste termo de correção, que será importante na discussão dos resultados não- 
colineares para os sistemas de $\mathrm{Pd} / \mathrm{Fe} / \operatorname{Ir}(111)$ e $\mathrm{Pd} / \mathrm{Co} / \operatorname{Ir}(111)$, no capítulo 5. Considere a equação de onda relativística proposta por Paul Dirac em 1928 [85] para um elétron $\left(\operatorname{spin} \frac{1}{2}\right.$, carga $-e$, e massa de repouso $m$ ) submetido a um potencial escalar $V(\mathbf{r})$ e a um potencial vetor $\mathbf{A}(\mathbf{r})$ :

$$
\mathcal{H} \psi(\mathbf{r})=i \hbar \frac{\partial}{\partial t} \psi(\mathbf{r})
$$

com

$$
\mathcal{H} \equiv-e V(\mathbf{r})+\beta m c^{2}+\boldsymbol{\alpha} \cdot[c \mathbf{p}+e \mathbf{A}(\mathbf{r})]
$$

Aqui $p$ representa o vetor momento, e $\boldsymbol{\alpha}$ um vetor de matrizes $4 \times 4$ construídas a partir das matrizes de Pauli $\boldsymbol{\sigma}, \boldsymbol{\alpha}=\left[\begin{array}{ll}0 & \boldsymbol{\sigma} \\ \boldsymbol{\sigma} & 0\end{array}\right]$, e $\beta=\left[\begin{array}{cc}\mathbb{1} & 0 \\ 0 & -\mathbb{1}\end{array}\right]$. As autofunções da Eq. 2.6 são os quadrivetores usualmente escritos como

$$
\psi(\mathbf{r})=\left[\begin{array}{l}
\varphi(\mathbf{r}) \\
\zeta(\mathbf{r})
\end{array}\right]
$$

em que os bivetores $\varphi(\mathbf{r})$ e $\zeta(\mathbf{r})$ são componentes da função de onda. Inserindo a Eq. $\mathbf{2 . 8}$ na Eq. 2.6 e utilizando a Eq. 2.7, obtêm-se duas equações diferenciais

$$
\begin{aligned}
& {\left[E-2 m c^{2}+e V(\mathbf{r})\right] \varphi(\mathbf{r})=\boldsymbol{\alpha} \cdot[c \mathbf{p}+e \mathbf{A}(\mathbf{r})] \zeta(\mathbf{r})} \\
& {\left[E+2 m c^{2}+e V(\mathbf{r})\right] \zeta(\mathbf{r})=\boldsymbol{\alpha} \cdot[c \mathbf{p}+e \mathbf{A}(\mathbf{r})] \varphi(\mathbf{r})}
\end{aligned}
$$

A substituição de uma expressão na outra permite elaborar a equação para a componente $\varphi(\mathbf{r})$, também chamada de equação de Pauli. Assumindo que $\mathbf{A}(\mathbf{r})=\mathbb{O}$, e supondo o limite de $v \ll c$, a equação de Pauli se reduz à relação de Schrödinger com um termo $\mathcal{H}_{S O}$ adicional na Hamiltoniana $(e=1, \hbar=1)$ :

$$
\mathcal{H}_{S O}=\frac{1}{4 m_{\mathrm{rel}}^{2} c^{2}} \boldsymbol{\sigma} \cdot(\nabla V(\mathbf{r}) \times \mathbf{p})
$$

em que a massa de repouso $m$ do elétron é substituída pela massa relativística $m_{\text {rel }}$. Se $\mathbf{m}_{l}$ é o momento orbital do elétron, então utilizando as transformações $\boldsymbol{\sigma} \cdot(\nabla V(\mathbf{r}) \times \mathbf{p})=$ $\frac{1}{r} \frac{d V(r)}{d r} \boldsymbol{\sigma} \cdot(\mathbf{r} \times \mathbf{p})=\frac{1}{r} \frac{d V(r)}{d r} \boldsymbol{\sigma} \cdot \mathbf{m}_{l}$ e $\mathbf{s} \equiv \frac{\boldsymbol{\sigma}}{2}$, a Eq. $\mathbf{2 . 1 0}$ resulta na expressão para o acoplamento spin-órbita:

$$
\mathcal{H}_{S O}=\frac{1}{2 m_{\mathrm{rel}}^{2} c^{2}} \frac{1}{r} \frac{d V(r)}{d r} \mathbf{m}_{l} \cdot \mathbf{s}
$$


Para elementos mais leves, $\mathbf{m}_{l}$ pode ser desprezível em comparação à contribuição do momento de spin. Já em elementos mais pesados, os efeitos relativísticos tornam-se gradativamente mais relevantes, aumentando a importância do acoplamento spin-órbita. Por sua vez, o coeficiente $\xi(r) \equiv \frac{1}{2 m_{\mathrm{rel}}^{2} c^{2}} \frac{1}{r} \frac{d V(r)}{d r}$ é denominado fator de strength (ou, simplesmente, constante de acoplamento spin-órbita). Nos cálculos autoconsistentes, o termo da Eq. 2.11 é adicionado à Hamiltoniana do problema em cada etapa variacional.

\subsection{Interação de Dzyaloshinskii-Moriya (DM)}

Na seção 2.1, introduziu-se o modelo de Heisenberg para o cálculo da interação de troca magnética entre os momentos localizados nos sítios genéricos $i$ e $j$. Uma importante propriedade desta interação é ser isotrópica devido à possibilidade de transformação $\mathbf{S}_{i} \cdot \mathbf{S}_{j}=\mathbf{S}_{j} \cdot \mathbf{S}_{i}$. Desta forma, por exemplo, as configurações magnéticas ( $\left.\downarrow\right)$ e $(\downarrow \uparrow)$ apresentam a mesma energia. Entretanto, Dzyaloshinskii [48] e Moriya [49] observaram que este acoplamento de troca pode conter, também, um termo anisotrópico. Baseado em argumentos de simetria e na teoria de Landau [86], Dzyaloshinskii previu uma correção no Hamiltoniano original da forma $\mathcal{H}^{\prime} \propto \mathbf{D}_{12} \cdot\left(\mathbf{S}_{1} \times \mathbf{S}_{2}\right)$, capaz de promover uma magnetização líquida não-nula em cristais ferromagnéticos com baixa simetria, tais como $\mathrm{MnCO}_{3}$ ou $\alpha-\mathrm{Fe}_{2} \mathrm{O}_{3}$. O modelo de Dzyaloshinskii, portanto, foi originalmente fenomenológico. Moriya, por sua vez, identificou o acoplamento spin-órbita como o mecanismo responsável por este termo extra antissimétrico, a partir da expansão da teoria de Anderson para o acoplamento de superexchange [87] visando incluir a interação spin-órbita. Assim, considerando todos os sítios $i$ e $j$ que compõem o material em questão, de modo similar à Eq. 2.2 pode-se escrever o termo $\mathcal{H}^{\prime} \equiv \mathcal{H}_{D M}$ como:

$$
\mathcal{H}_{D M}=\sum_{i \neq j} \mathbf{D}_{i j} \cdot\left(\mathbf{S}_{i} \times \mathbf{S}_{j}\right)
$$

intrinsecamente distinto à Hamiltoniana de Heisenberg, pois $\mathbf{S}_{i} \times \mathbf{S}_{j}=-\mathbf{S}_{j} \times \mathbf{S}_{i} . \mathrm{Na}$ equação acima, $\mathbf{D}_{i j}$ é denominado vetor de Dzyaloshinskii-Moriya. Sua relação com o acoplamento spin-órbita é brevemente explorada no Apêndice A.

A interação de DM está no âmago do processo de formação de texturas complexas de spin, essencialmente por desestabilizar os estados colineares e favorecer estruturas magnéticas construídas em torno de um eixo preferencial de rotação [88]. Entre elas, pode-se citar: ( $i$ ) espirais de spin [89, 90]; e (ii) skyrmions [26, 46, 60, 91] (seção 2.6), que serão amplamente explorados neste trabalho. Em cristais que apresentam simetria de 
inversão, tais como os materiais bulk mais simples, o acoplamento do tipo DM é suprimido, e o termo de exchange isotrópico é dominante [92]. Por outro lado, em superfícies ou outros sistemas cuja simetria de inversão é quebrada [93], é possível que sua intensidade se torne relativamente importante, em especial quando induzida por espécies atômicas com elevado acoplamento spin-órbita. Neste sentido, uma das propostas da literatura é a análise da razão $\frac{\mathcal{J}}{D}[91]$ - na qual $D \equiv\left|\mathbf{D}_{i j}\right|$ é a constante de Dzyaloshinskii - capaz de fornecer uma explicação qualitativa preliminar para a eventual configuração não-colinear verificada para o sistema.

\section{$2.6 \quad$ Skyrmions}

O conceito de skyrmion originou-se do trabalho de Tony Skyrme [94], na década de 1960. Partindo de suas investigações em teoria não-linear de campos para píons interagentes, Skyrme demonstrou que uma das soluções esse tipo de teoria englobava configurações quantizadas e topologicamente protegidas em meio a um campo contínuo, e que apresentavam um caráter tipo-partícula. Embora essa ideia tivesse se originado de uma tentativa de explicação para a existência de prótons e nêutrons, a solução de Skyrme foi posteriormente adaptada para diversas áreas da Física, envolvendo desde o estudo de partículas elementares até cristais líquidos, condensados de Bose-Einstein, e gases bidimensionais que exibem o efeito Hall quântico [95].

Em poucas palavras, no contexto do nanomagnetismo podemos definir os skyrmions como pequenos defeitos topológicos na textura (não-colinear) magnética do material, para os quais há um sentido único de rotação dos spins. Assim, é importante ressaltar que os skyrmions caracterizam-se como estruturas magnéticas locais. Uma ilustração representativa da estrutura de um skyrmion é apresentada na Figura 2.3. Na maioria dos sistemas de estado sólido, essa configuração de spins é determinada e estabilizada por interações anisotrópicas do tipo de DM, e sua competição com os acoplamentos de troca $\left(\mathcal{J}_{i j}\right)$ entre os diferentes sítios no material [96]. Portanto, embora existam outras espécies de texturas magnéticas não-colineares locais que podem ser estabilizadas sem o auxílio das interações de $\mathrm{DM}^{1}$, uma diferença fundamental desse tipo de estrutura é que não há uma quiralidade bem definida, base de muitas das propriedades topológicas dos skyrmions [98]. Assim, é usual classificar os skyrmions em duas categorias distintas: $(i)$ os do tipo de Néel (Fig. 2.4(a)); e (ii) os do tipo de Bloch (Fig. 2.4(b)), ambos com diferenças nas simetrias das interações entre spins, que resultam em sentidos distintos de rotação. Para cada uma

\footnotetext{
${ }^{1}$ Podem ser, por exemplo, estabilizadas apenas por interações dipolares, de mais longo alcance [97].
} 
dessas disposições dos spins no espaço associa-se uma quantização inteira denominada carga topológica, que será abordada de forma mais detalhada na seção 2.6.1.

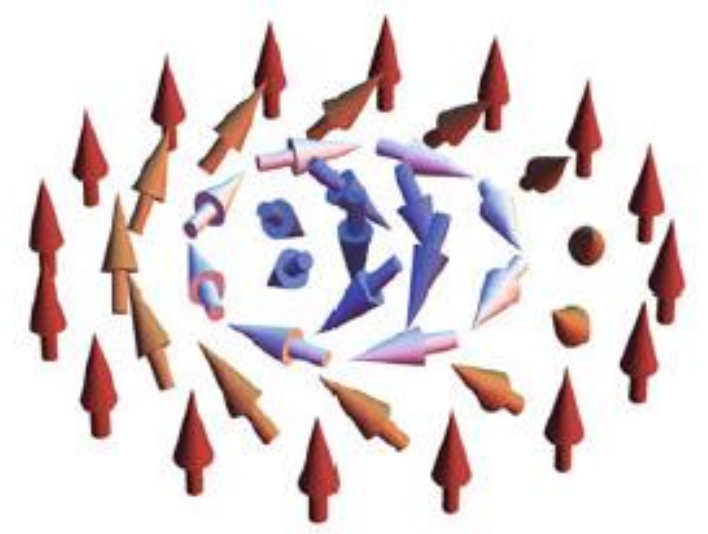

Figura 2.3: Estrutura de um skyrmion bidimensional, tal como aqueles encontrados em sistemas de estado sólido. As setas representam as direções locais dos spins nos sítios atômicos. Figura retirada da referência [99].
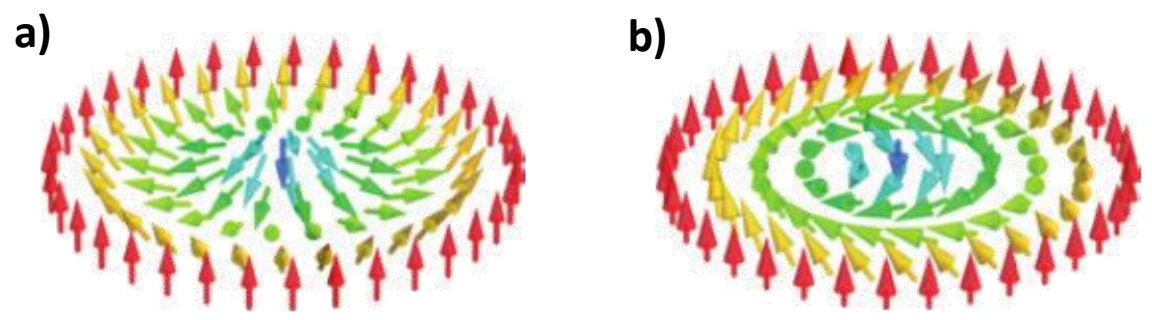

Figura 2.4: Configurações de skyrmions observadas em sistemas magnéticos, em dois tipos distintos: (a) de Néel, cuja rotação dos spins ocorre em um plano perpendicular à direção radial; e (b) de Bloch, cuja rotação dos spins ocorre no plano radial. Os skyrmions do tipo de Néel comumente surgem em sistemas nos quais as interações de DM ocorrem na interface entre dois materiais, isto é, organizados em multicamadas [100]. Figura adaptada da referência [95].

Em condições ideais, os skyrmions se manifestam em redes regulares de texturas circulares ou quase circulares, demonstrando uma curiosa semelhança com a estrutura de vórtices encontrada nos supercondutores do tipo-II, quando na presença de um campo magnético externo [101]. Embora sua existência estivesse inicialmente associada apenas à condições de não-equilíbrio no sistema - tais como a aplicação de campos magnéticos externos e defeitos topológicos -, Rössler et al. [57] mostraram pela primeira vez em 2006, utilizando um modelo fenomenológico, que skyrmions podem ocorrer espontaneamente como estado magnético fundamental em determinados materiais, desde que esses sistemas apresentem uma quebra espontânea de simetria de inversão. Em especial, essa quebra de simetria é intrínseca a sistemas com dimensionalidade reduzida, tais como filmes finos, monocamadas (multicamadas) e nanoestruturas [23]. Como previsto, skyrmions 
espontâneos foram observados experimentalmente para uma monocamada de Fe/Ir(111) $[46,102]$.

Mas o que tem chamado tanto a atenção de parte da comunidade científica da área de nanomagnetismo para essas estruturas com características topológicas? A resposta para essa pergunta reside no fato de que os skyrmions podem ser facilmente conduzidos pelo material com a aplicação de correntes elétricas com baixas densidades $\left(\sim 10^{6} \mathrm{~A} / \mathrm{m}^{2}\right)[25$, 26], cerca de $10^{5}$ vezes menos intensas do que as densidades de corrente típicas necessárias para se movimentar paredes de domínio [25, 28]. E é justamente na movimentação dos spins (ao invés da movimentação física do próprio material) que está a grande aposta da spintrônica para os novos dispositivos de gravação magnética: os atuais discos rígidos (HDDs), por exemplo, apresentam um custo econômico atrativo, mas uma severa limitação pelo fato de eles serem intrinsecamente lentos, com tempos de acesso típicos da ordem de milissegundos [28]. Essa movimentação de regiões limitadas de magnetização, ou de texturas complexas locais de spin como os skyrmions ocorre essencialmente por ação do efeito conhecido como Spin Transfer Torque (STT) [103]. Quando um elétron de condução passa através dessas estruturas magnéticas, seu momento de spin adapta-se constantemente à magnetização local conduzido pelas interações com os demais spins do ambiente (Figura 2.5), induzindo forças que atuam tanto na estrutura magnética quanto no próprio elétron; um termo adicional na equação de Landau-Lifshitz-Gilbert (LLG) foi incluído por Slonczewski em 1996 [104] para levar em conta o efeito de STT. Assim, por conservação de momento angular, a diferença entre as direções do momento de spin de um elétron incidente e da magnetização local gera um torque, sendo que ambas as direções sofrem uma rotação [103]. Isso torna possível movimentar um skyrmion por meio da aplicação de uma corrente elétrica no sistema sem dificuldade; por outro lado, o fato de os skyrmions exibirem propriedades topológicas favorece uma dinâmica sem que ocorra alterações em sua morfologia. Essa característica é particularmente interessante, pois torna viável o projeto e a construção de memórias do tipo racetrack baseadas em skyrmions, nas quais cada estrutura topológica pode representar um bit de informação [27, 105]. Ademais, a emergência de um campo magnético local (i.e. confinado) da ordem de $100 \mathrm{~T}$ [106] é uma propriedade fundamental dos skyrmions, e que torna possível a sua leitura. 


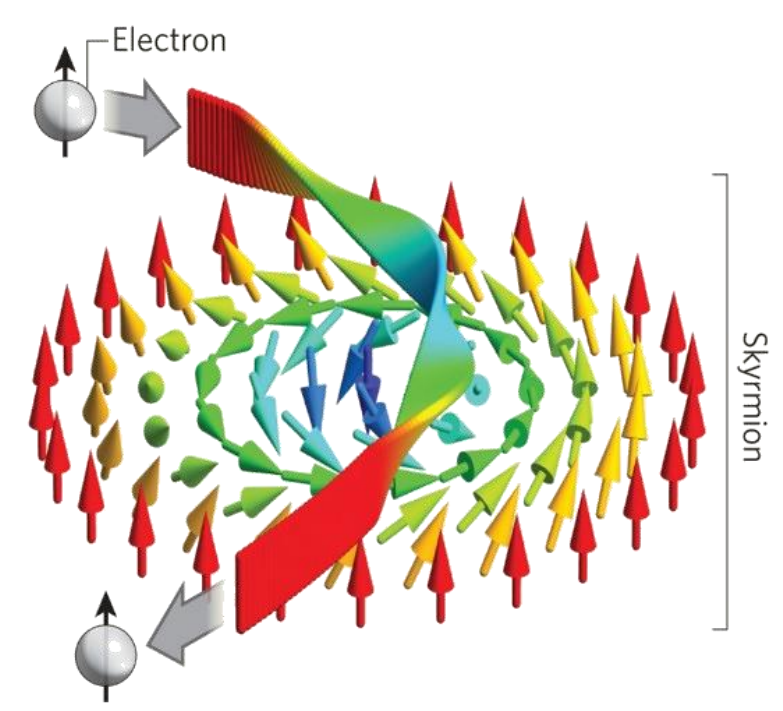

Figura 2.5: Variação do momento de spin de um elétron ao passar por um skyrmion. A direção do momento é representada por um código de cores, que vai do vermelho (orientação para cima) ao azul (orientação para baixo). Figura retirada da referência [107].

Experimentalmente, os skyrmions já foram obtidos tanto em sistemas do tipo bulk [42, 108] quanto em sistemas organizados em multi/monocamadas [52, 55, 96, 98, 109, 110]. Como já mencionado, S. Heinze et al. [46] observaram a formação de uma rede espontânea de skyrmions em uma monocamada de Fe depositada em $\operatorname{Ir}(111)$, atribuindo sua origem microscópica à atuação conjunta das interações de troca, DM, e four-spin ${ }^{1}$. A mesma rede de skyrmions serviu como suporte para a mediação de um acoplamento de longo alcance (da ordem de nanômetros) entre compostos orgânicos individuais depositados sobre a monocamada de Fe/Ir(111) [102]. Por outro lado, Romming et al. [52] obtiveram o importante controle local de criação e aniquilação de skyrmions introduzindo pequenas correntes elétricas através de uma ponta de STM com polarização de spin (SP-STM) [13]. Apesar de demonstrarem a possibilidade de gravação de informação utilizando skyrmions, a bicamada de $\mathrm{Pd} / \mathrm{Fe}$ depositada em $\operatorname{Ir}(111)$ exibe uma fase com essas estruturas magnéticas apenas à baixas temperaturas, da ordem de $T=4,2 \mathrm{~K}$. Nesse sentido, Wiesendanger [96] recentemente apresentou em seu trabalho um resumo dos principais sistemas bidimensionais nos quais skyrmions foram encontrados, sempre emergindo à baixas temperaturas, quando possuem pequenas dimensões (ideal), ou com um tamanho muito grande, quando estabilizados à temperatura ambiente.

Por fim, em relação à reprodutibilidade experimental dos skyrmions, Jiang et al. [110]

\footnotetext{
[51].

${ }^{1}$ Termo na Hamiltoniana de spin que representa o efeito do hopping eletrônico em 4 sítios adjacentes
} 
propuseram um método bastante eficaz. Partindo da geração de domínios magnéticos na forma de fitas na multicamada de $\mathrm{Ta}(5 \mathrm{~nm}) / \mathrm{Co}_{20} \mathrm{Fe}_{60} \mathrm{~B}_{20}(1,1 \mathrm{~nm}) / \mathrm{TaO}_{x}(3 \mathrm{~nm})$, e da aplicação de uma densidade de corrente não-homogênea provocada por uma constrição geométrica no material, os autores demonstraram ser possível formar skyrmions como resultado de forças efetivas (associado ao efeito Hall de spin [111]) nas bordas desses domínios. Assim, por serem topologicamente protegidos, os recém-formados skyrmions mantêm sua estrutura e são movimentados graças à própria densidade de corrente aplicada ao material. Esse método apresenta grande semelhança com a formação de bolhas a partir de filmes finos de sabão, e mostra-se muito mais simples quando comparado ao método de criação/aniquilação proposto por Romming et al. [52], dois anos antes.

As pesquisas mais recentes têm mostrado que, para materiais com anisotropia cúbica, há a possibilidade de existir duas diferentes fases de skyrmions para a mesma amostra, termodinamicamente desconexas [112].

Essa breve análise da literatura exemplifica a importância dos skyrmions no âmbito de pesquisa de fronteira em magnetismo e permite esboçar a lacuna existente na literatura sobre o conhecimento dos sistemas e condições ideais para a emergência desse tipo de estrutura. No presente trabalho, nosso foco será voltado, em um primeiro momento, à pesquisa de tais condições (tomando como base duas bicamadas modelo), e, em um segundo momento, à investigação de materiais com propriedades interessantes visando novas propostas para futuras aplicações em dispositivos de gravação magnética.

\subsubsection{Carga topológica $q$}

Os skyrmions na Física dos Materiais, tal como mencionado anteriormente, são definidos na forma de estruturas magnéticas complexas (não-triviais), conhecidas - em conjunto com outras entidades físicas, como os sólitons e as paredes de domínio - como defeitos topológicos, justamente porque sua estabilidade por ser compreendida a partir de argumentos de topologia. Assim, com a finalidade de estabelecer de forma mais concreta como os conceitos de topologia se inserem no contexto de materiais magnéticos, introduziremos a chamada carga topológica $q$, um número inteiro associado a cada skyrmion que pode ser interpretado como a quantidade de voltas que a direção de magnetização local dá ao redor de uma esfera de raio unitário (esfera de Bloch).

Certamente, a interpretação matemática de $q$ não é simples à primeira vista, mas podemos imaginar as seguintes situações. Um estado ferromagnético puro, dito trivial sob o ponto de vista de topologia, é caracterizado pelo alinhamento dos momentos magnéticos 
de spin a uma única direção no espaço. Neste caso, ao tentarmos "preencher" a esfera de Bloch com as diferentes orientações que os spins podem assumir ao longo de toda a extensão do material, permaneceremos em um único ponto da esfera. Dessa forma e pela definição, o estado ferromagnético é caracterizado pela carga topológica $q=0$, visto que a magnetização do sistema não foi suficiente para completar nenhuma volta na esfera de Bloch.

O mesmo pode ocorrer para texturas não-colineares de spin. Nesse sentido, suponha que um sistema apresenta a configuração magnética com os spins arranjados em uma espiral. Agora, ao contrário do que ocorre com o estado ferromagnético, os spins locais assumem diferentes direções ao longo da extensão do material, mas que não são suficientes para preencher um número inteiro de voltas na esfera de Bloch. Por esse motivo, considerase também a espiral de spin como um estado magnético trivial, caracterizado pela carga $q=0$. Por outro lado, os skyrmions, por se tratarem de estruturas nas quais há um senso único de rotação em todas as direções, apresentam $q \neq 0$ - e essa diferença permite classificar a carga topológica como uma boa quantidade para se caracterizar a presença de skyrmions em um sistema. Uma representação esquemática do preenchimento de spins pertencentes a um único skyrmion na esfera unitária é apresentada na Figura 2.6. Explicitamente, a carga $q$ pode ser definida como

$$
q=\frac{1}{4 \pi} \iint_{S}\left(\frac{\partial \hat{\mathbf{n}}}{\partial x} \times \frac{\partial \hat{\mathbf{n}}}{\partial y}\right) \cdot \hat{\mathbf{n}} d \mathbf{s}
$$

onde $\hat{\mathbf{n}}$ é um vetor unitário paralelo à direção local do spin e $d \mathbf{s}$ é o elemento de área. ${ }^{1}$

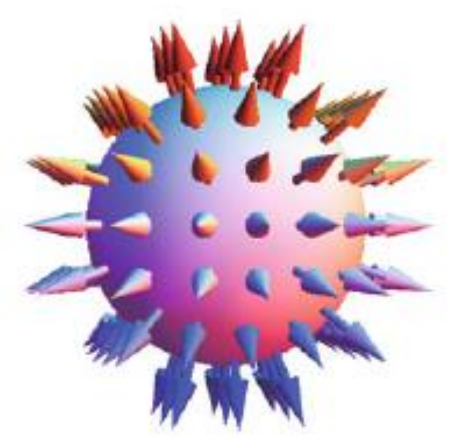

Figura 2.6: Representação de um skyrmion do tipo "hedge-hog" tal como proposto inicialmente por Tony Skyrme na década de 1960. Nele, a magnetização aponta para todas as direções na esfera unitária. A projeção dessa configuração em duas dimensões gera o skyrmion definido na Fig. 2.3. Figura reproduzida da referência [99].

\footnotetext{
${ }^{1}$ Note que essa definição é válida tanto para skyrmions que surgem em materiais bidimensionais como tridimensionais, sendo que, para estes últimos, deve-se considerar a sua projeção no plano.
} 
Embora tenhamos apresentado uma definição formal da carga topológica na Eq. 2.13, em um material real - sobretudo se estamos investigando pequenas regiões (da ordem de nanômetros ou menos) - a magnetização $\mathbf{m}(x, y, z)=\mathbf{m}(\mathbf{r})$ deve ser tratada como um campo vetorial discreto, no qual os spins localizam-se em posições bem definidas no espaço. Isto é, grosso modo, não é mais correto realizar o cálculo de $q$ pela sua definição para sistemas contínuos. Pensando nisto, B. Berg e M. Lüscher [113] resolveram esse impasse utilizando o seguinte raciocínio. Antes de prosseguir, porém, cabe destacar que os autores da referência [113] consideraram um sistema plano de spins configurado como uma rede quadrada (grupo pontual $\left.C_{4 h}\right)^{1}$; entretanto, conforme mostraremos mais adiante, a mesma dedução pode ser expandida para qualquer matriz de spins que preencha o espaço bidimensional por completo (redes de Bravais), porém com alguns cuidados.

Como no modelo contínuo (Eq. 2.13), a carga topológica total $Q$ de uma rede discreta de spins pode ser obtida pela soma de todas as densidades topológicas locais $q\left(x^{*}\right)$, isto é

$$
Q=\sum_{x^{*}} q\left(x^{*}\right)
$$

em que essa quantidade $q\left(x^{*}\right)$ está vinculada ao sítio $x^{*}$ e aos spins localizados em sua primeira vizinhança, como pode ser visto na Figura 2.7.

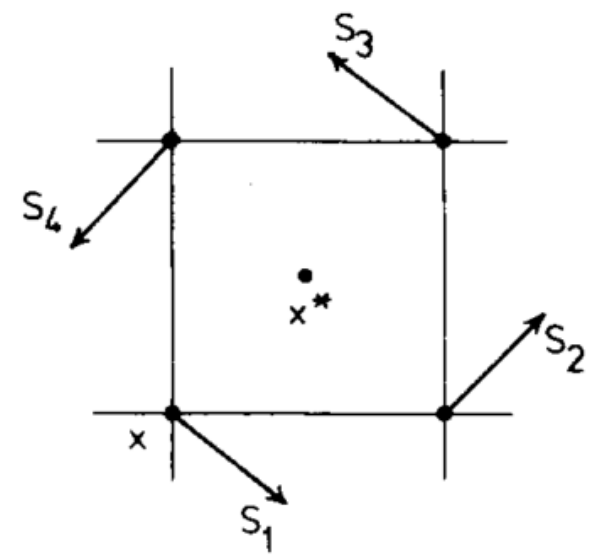

Figura 2.7: Representação do sítio $x^{*}$ para uma rede quadrada, localizando-se no centro da primeira vizinhança com spins $\mathbf{s}_{1}, \mathbf{s}_{2}, \mathbf{s}_{3}$, e $\mathbf{s}_{4}$. Figura reproduzida da referência [113].

Mais precisamente, para uma rede quadrada, $q\left(x^{*}\right)$ será uma função dos quatro spins

\footnotetext{
${ }^{1}$ Sendo nossos materiais de interesse bidimensionais, o desenvolvimento matemático nesta seção será realizado considerando-se uma rede plana de spins.
} 
$\mathbf{s}_{1}, \mathbf{s}_{2}, \mathbf{s}_{3}, \mathbf{s}_{4}$ primeiros vizinhos de $x^{*}$ :

$$
q\left(x^{*}\right)=\frac{1}{4 \pi}\left[(\sigma A)\left(\mathbf{s}_{1}, \mathbf{s}_{2}, \mathbf{s}_{3}\right)+(\sigma A)\left(\mathbf{s}_{1}, \mathbf{s}_{3}, \mathbf{s}_{4}\right)\right],
$$

onde $(\sigma A)\left(\mathbf{s}_{1}, \mathbf{s}_{2}, \mathbf{s}_{3}\right)$, por exemplo, denota a área do triângulo esférico com vértices $\mathbf{s}_{1}, \mathbf{s}_{2}$, e $\mathbf{s}_{3}$ (veja a Figura 2.8). Em outras palavras, a quantidade $4 \pi Q$ define a área total da superfície formada pela junção de todos os triângulos esféricos elementares com vértices $s_{1}\left(x^{*}\right), s_{2}\left(x^{*}\right), s_{3}\left(x^{*}\right)$, e $s_{1}\left(x^{*}\right), s_{3}\left(x^{*}\right), s_{4}\left(x^{*}\right)$, respectivamente.

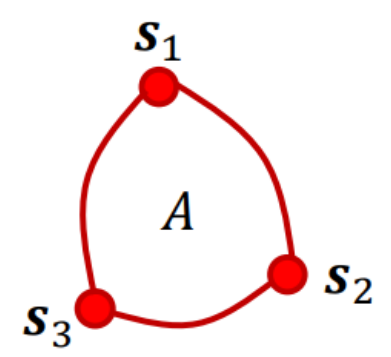

Figura 2.8: Representação de um triângulo esférico com área $A$ formado pelos spins $\mathbf{s}_{1}$, $\mathbf{s}_{2}$, e $\mathbf{s}_{3}$.

É justamente nesta definição que reside a possibilidade de expandirmos a dedução do cálculo de $q$ para qualquer rede de Bravais bidimensional. Para um polígono convexo de $n$ lados formado a partir da primeira vizinhança de spin do sítio $x^{*}$, sua área total pode ser expressa como uma soma de $(n-2)$ triângulos, bastando ordenar ciclicamente $\mathbf{s}_{1}, \mathbf{s}_{2}, \ldots, \mathbf{s}_{n}$ de tal maneira que as funções $(\sigma A)$ estejam associadas a triângulos distintos. Portanto, $q\left(x^{*}\right)$ (Eq. 2.15) será calculado como uma soma de $(n-2)$ termos, ao invés de apenas dois. Cabe ressaltar também que a carga topológica total $Q$ não é bem determinada para configurações exageradamente excêntricas de $\operatorname{spin}^{1}$, mas em geral caracteriza-se como um observável bem definido [113]. Logo, para o caso de uma rede hexagonal, pode-se generalizar a Eq. 2.15 para:

$$
q\left(x^{*}\right)=\frac{1}{4 \pi}\left[(\sigma A)\left(\mathbf{s}_{1}, \mathbf{s}_{2}, \mathbf{s}_{3}\right)+(\sigma A)\left(\mathbf{s}_{1}, \mathbf{s}_{3}, \mathbf{s}_{4}\right)+(\sigma A)\left(\mathbf{s}_{1}, \mathbf{s}_{4}, \mathbf{s}_{5}\right)+(\sigma A)\left(\mathbf{s}_{1}, \mathbf{s}_{5}, \mathbf{s}_{6}\right)\right] .
$$

Uma expressão explícita para a área $(\sigma A)\left(\mathbf{s}_{1}, \mathbf{s}_{2}, \mathbf{s}_{3}\right)$ não é difícil de se obter, para quaisquer que sejam os spins $\mathbf{s}_{1}, \mathbf{s}_{2}$, e $\mathbf{s}_{3}$. Sabe-se que a área $A$ de um triângulo esférico

\footnotetext{
${ }^{1}$ Como veremos, por exemplo, no caso da fase paramagnética da bicamada de $\mathrm{Pd} / \mathrm{Fe} / \mathrm{Ir}(111)$.
} 
com ângulos internos $\alpha_{1}, \alpha_{2}, \alpha_{3}$ (em uma esfera de raio unitário) é escrita diretamente a partir do excedente angular

$$
A=\alpha_{1}+\alpha_{2}+\alpha_{3}-\pi
$$

com orientação definida por $\sigma=\operatorname{sinal}\left[\mathbf{s}_{1} \cdot\left(\mathbf{s}_{2} \times \mathbf{s}_{3}\right)\right]= \pm 1$ (para $\left.A \neq 0,2 \pi\right)$. Longe dos arranjos não-triviais, temos que $\mathbf{s}_{1} \cdot\left(\mathbf{s}_{2} \times \mathbf{s}_{3}\right)=0$, o que estende a função $(\sigma A)\left(\mathbf{s}_{1}, \mathbf{s}_{2}, \mathbf{s}_{3}\right)$ para uma função analítica e real cujos valores de $A$ podem variar no intervalo $-2 \pi<$ $A<2 \pi$. Combinando, então, as relações derivadas para $\sigma$ e $A$ (Eq. 2.17), chegamos na expressão final que relaciona a área com os três spins $\mathbf{s}_{1}, \mathbf{s}_{2}$, e $\mathbf{s}_{3}$ de um triângulo esférico [113]:

$$
\exp \left(\frac{1}{2} i \sigma A\right)=\frac{1}{\rho}\left[1+\mathbf{s}_{1} \cdot \mathbf{s}_{2}+\mathbf{s}_{2} \cdot \mathbf{s}_{3}+\mathbf{s}_{3} \cdot \mathbf{s}_{1}+i \mathbf{s}_{1} \cdot\left(\mathbf{s}_{2} \times \mathbf{s}_{3}\right)\right]
$$

na qual $\rho=\sqrt{2\left(1+\mathbf{s}_{1} \cdot \mathbf{s}_{2}\right)\left(1+\mathbf{s}_{2} \cdot \mathbf{s}_{3}\right)\left(1+\mathbf{s}_{3} \cdot \mathbf{s}_{1}\right)}>0$. Logo, existindo uma relação explícita para $(\sigma A)\left(\mathbf{s}_{1}, \mathbf{s}_{2}, \mathbf{s}_{3}\right)$, temos naturalmente uma expressão para $q\left(x^{*}\right)$ e também para a carga total $Q$ do sistema, seguindo a Eq. 2.14. Conforme mencionado anteriormente, a fim de se obter $Q$ em uma rede não-quadrada, é suficiente estender a definição da Eq. 2.15 para levar em conta toda a primeira vizinhança formada pelos spins $\mathbf{s}_{1}, \mathbf{s}_{2}, \ldots, \mathbf{s}_{n}$.

Na prática, para o cálculo de $Q$ em uma rede de spins, podemos considerar $x^{*}$ como sendo cada sítio não-equivalente dessa rede, apenas tomando o cuidado de dividir o somatório final por um fator inteiro $m$ que representa quantas vezes a área determinada pela primeira vizinhança de $x^{*}$ foi contada repetidamente. Assim, para uma rede quadrada, e seguindo esta definição, $m=2$. Já para uma rede hexagonal, temos que $m=3$. Um programa em Python3 foi implementado para o cálculo de $Q$, e suporta tanto redes quadradas como hexagonais (a simetria dos sistemas de spins de interesse no presente trabalho). As direções locais dos spins obtidas de cada passo da simulação dinâmica tornam possível também calcular a carga topológica total $Q$ como uma função do tempo, isto é, $Q=Q(t)$. Assim, o desenvolvimento deste código em Python3, bem como a generalização da Eq. 2.15 para uma rede hexagonal são parte dos resultados obtidos no presente trabalho.

A partir da quantidade $q\left(x^{*}\right)$ podemos construir mapas de densidades de skyrmions, que mostram as regiões mais prováveis no sistema spins nas quais podem existir esse tipo de estrutura magnética. A Figura 2.9, abaixo, apresenta uma comparação entre um sistema de spins modelo (rede quadrada) e sua densidade calculada de skyrmions, 
utilizando a definição da Eq. 2.15. É importante notar que, para o caso da Fig. 2.9(a), obtivemos o valor $Q=-20$, indicando a existência de 20 skyrmions com carga topológica individual $q=-1$.
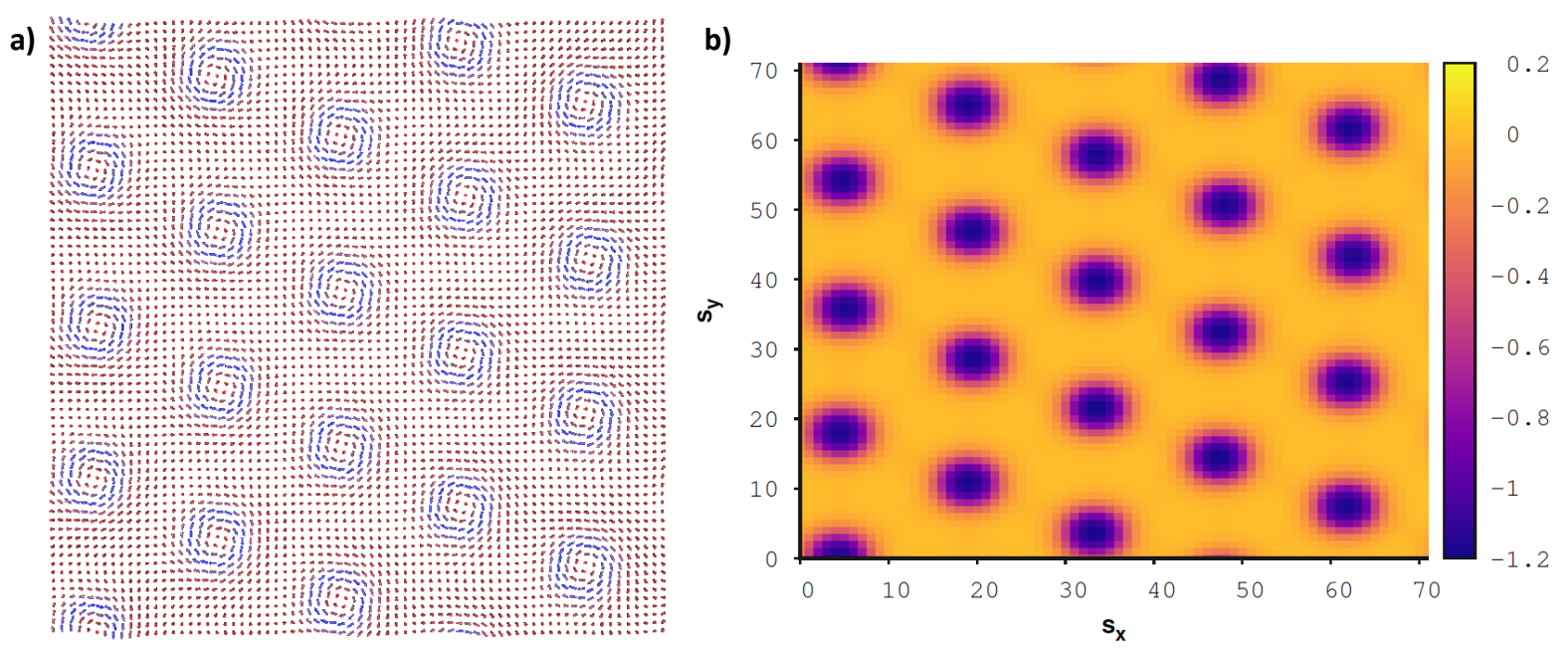

Figura 2.9: Comparação entre: (a) matriz quadrada composta por $72 \times 72$ spins, com parâmetros fictícios $\mathcal{J}=10,88 \mathrm{meV},|\mathbf{D}|=4,31 \mathrm{meV}$ (interações apenas entre primeiros vizinhos), e $B=55 \mathrm{~T}$ (aplicado perpendicularmente ao plano); e (b) densidade $q\left(x^{*}\right)$ calculada para todos os sítios do sistema utilizando a definição da Eq. 2.15.

\subsection{Energia de anisotropia magnética (MAE)}

Os cristais - e particularmente também os sistemas de menor dimensão - possuem eixos de fácil e de difícil magnetização. Isto é, ao longo de determinadas direções cristalográficas é necessário um menor ou maior campo B aplicado para que o sistema seja magnetizado. A Fig. 2.10 mostra este efeito para os cristais de Fe, Co, e Ni. Essa anisotropia magnética pode ter diversas origens: o formato da amostra, o stress no material, a segregação atômica, entre outras. Em todas as suas variações conhecidas (uniaxial, cúbica, e outros), o termo adicional de energia (MAE) vinculado à existência de eixos preferenciais de alinhamento dos momentos magnéticos depende exclusivamente do ângulo relativo ao eixo de fácil magnetização, e deve ser invariante com a troca de sinal dos cossenos diretores que o compõem. Logo, as potências ímpares destes cossenos não podem aparecer na expressão final. Além disso, as permutações entre os cossenos devem resultar na mesma energia de anisotropia [114]. 

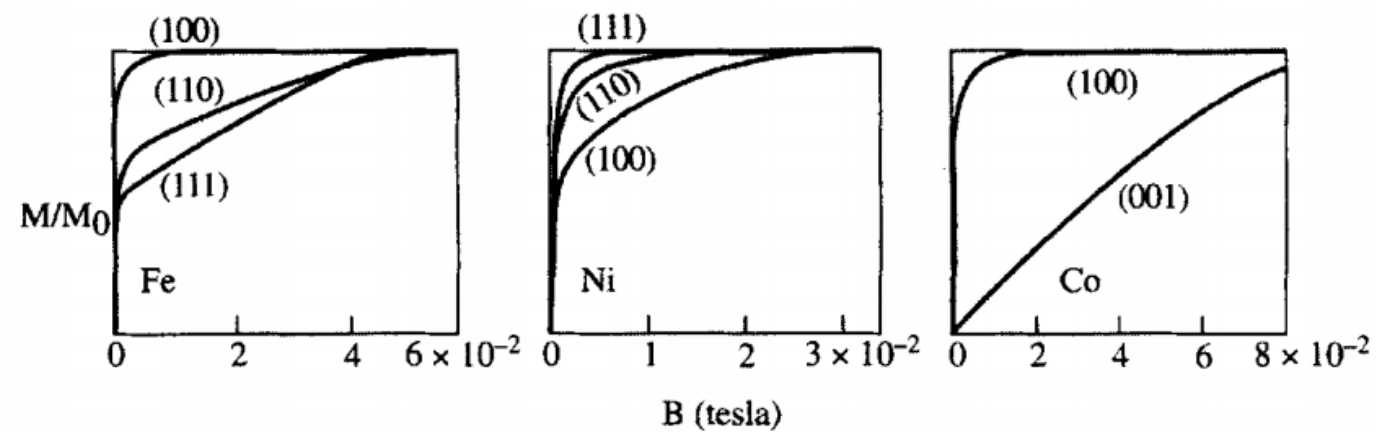

Figura 2.10: Magnetização relativa nos bulks de Fe (bcc), Co $(h c p)$ e Ni $(f c c)$ para campos externos aplicados em diferentes direções, demonstrando a existência da anisotropia magnética. Figura retirada da referência [114].

Em determinados materiais, supõe-se válida a aproximação de que a MAE é uma função apenas do ângulo $\theta$ entre a direção de magnetização e um dado eixo considerado; trata-se, portanto, da anisotropia uniaxial. Esta aproximação descreve bem sistemas cristalinos hcp, tais como o bulk de Co [114]. Nela, a energia total pode ser expressa da seguinte forma [115-117]:

$$
E(\theta) \equiv \sum_{n=0}^{\infty} K_{n} \operatorname{sen}^{2 n}(\theta) \sim K_{0}+K_{1} \operatorname{sen}^{2}(\theta)+K_{2} \operatorname{sen}^{4}(\theta)
$$

onde os coeficientes $K_{n}$ têm dimensão de energia. Note que os termos proporcionais a $\operatorname{sen}^{6}(\theta), \operatorname{sen}^{8}(\theta), \ldots$, são desprezíveis para ângulos $\theta$ pequenos e foram desconsiderados na Eq. 2.19.

A energia de anisotropia surge pelo fato de as funções de onda atômicas dos íons magnéticos, quando em um meio no qual há acoplamento spin-órbita, muitas vezes não apresentarem simetria esférica, tais como os orbitais $d$ nos metais de transição. Em decorrência disto, os orbitais tendem a se acomodar segundo o campo cristalino ao qual o sítio em questão está sujeito. Definem-se, assim, certas direções preferenciais ao alinhamento dos momentos magnéticos elementares. Localmente, os spins sentem a anisotropia por meio da interação spin-órbita (seção 2.4), que os acopla ao movimento orbital de cada elétron.

Em cálculos de primeiros princípios, a MAE pode ser obtida de três principais maneiras, a saber:

- pela diferença de energia total (E) para o mesmo sistema calculado em duas diferentes direções de magnetização $\left(\hat{\mathbf{n}}_{\mathbf{1}}\right.$ e $\hat{\mathbf{n}}_{\mathbf{2}}$, por exemplo): $E_{\mathrm{MAE}}=E_{\hat{\mathbf{n}}_{\mathbf{1}}}-E_{\hat{\mathbf{n}}_{\mathbf{2}}}$; 
- pelo teorema do torque [118], em que:

$$
E_{\mathrm{MAE}}=T\left(\theta=45^{\circ}\right)=\left.\sum_{\text {occ. }}\left\langle\psi_{i, \mathbf{k}}^{\mathrm{SO}}\left|\frac{\partial H_{\mathrm{SO}}}{\partial \theta}\right| \psi_{i, \mathbf{k}}^{\mathrm{SO}}\right\rangle\right|_{\theta=45^{\circ}}
$$

onde $H_{\mathrm{SO}}$ e $\psi^{\mathrm{SO}}$ denotam a Hamiltoniana e a função de onda do sistema considerandose o acoplamento spin-órbita; e

- a partir da proporcionalidade da MAE com os valores de momento orbital nas duas direções de magnetização calculadas ( $\hat{\mathbf{n}}_{\mathbf{1}}$ e $\hat{\mathbf{n}}_{\mathbf{2}}$, por exemplo) [119, 120]:

$$
E_{\mathrm{MAE}}=\frac{-\xi}{4 \mu_{B}} \Delta m_{L}=\frac{-\xi}{4 \mu_{B}}\left(m_{L_{\hat{\mathbf{n}}_{1}}}-m_{L_{\hat{\mathbf{n}}_{2}}}\right)
$$

onde $m_{L_{\hat{\mathbf{n}}_{1}}}$ e $m_{L_{\hat{\mathbf{n}}_{2}}}$ são as projeções do momento orbital para dois diferentes eixos globais de quantização $\hat{\mathbf{n}}_{\mathbf{1}}$ e $\hat{\mathbf{n}}_{\mathbf{2}}$, $\xi$ é a constante de acoplamento spin-órbita (em unidades de energia) ${ }^{1}$, e $E_{\mathrm{MAE}}$ é a própria energia de anisotropia magnética (MAE), ou seja, a barreira energética para se mudar de um estado a outro. Um dos principais pressupostos para se derivar essa relação é que os elementos diagonais de spin da matriz associada ao acoplamento spin-órbita devem dominar a contribuição à MAE $[121]$.

\footnotetext{
${ }^{1}$ Não se observa variação significativa de $\xi$ quando é modificado o eixo principal de magnetização.
} 


\section{Capítulo 3}

\section{O método RS-LMTO-ASA}

\subsection{Teoria do Funcional da Densidade e métodos apro- ximativos}

A mecânica quântica estabelece que o comportamento estacionário microscópico da matéria, no caso não-relativístico, pode ser obtido por meio da solução da equação independente do tempo de Schrödinger:

$$
\mathcal{H} \Psi(\mathbf{r}, \mathbf{R})=E \Psi(\mathbf{r}, \mathbf{R})
$$

onde $\Psi(\mathbf{r}, \mathbf{R})$ representa a função de onda sujeita às posições eletrônicas $\mathbf{r} \equiv\left(\mathbf{r}_{1}, \mathbf{r}_{2}, \ldots, \mathbf{r}_{N}\right)$ e nucleares $\mathbf{R} \equiv\left(\mathbf{R}_{1}, \mathbf{R}_{2}, \ldots, \mathbf{R}_{M}\right)$, $E$ simboliza a energia total, e $\mathcal{H}$ é um Hamiltoniano de muitos corpos, que concentra os termos cinético e potencial associados aos núcleos e elétrons de um sistema poliatômico. Definindo $i, j$ como os índices para os $N$ elétrons desse sistema, e $A, B$ os índices relativos aos $M$ núcleos, então a Hamiltoniana $\mathcal{H}$ pode 
ser descrita pela seguinte expressão (em unidades atômicas de Rydberg ${ }^{1}$, u.a.):

$$
\begin{aligned}
& \mathcal{H}=\underbrace{-\sum_{i=1}^{N} \frac{1}{2} \nabla_{i}^{2}}_{\begin{array}{c}
\text { Operador de } \\
\text { energia cinética } \\
\text { dos elétrons }
\end{array}}-\underbrace{-\sum_{A=1}^{M} \frac{1}{2 M_{A}} \nabla_{A}^{2}}_{\begin{array}{c}
\text { Operador de } \\
\text { energia cinética } \\
\text { dos núcleos }
\end{array}}-\underbrace{\sum_{i=1}^{N} \sum_{A=1}^{M} \frac{Z_{A}}{\left|\mathbf{r}_{i}-\mathbf{R}_{A}\right|}}_{\begin{array}{c}
\text { Termo de } \\
\text { interacão eletrostática } \\
\text { elétron-núcleo }
\end{array}} \\
& \underbrace{+\sum_{i=1}^{N} \sum_{j>i}^{N} \frac{1}{\left|\mathbf{r}_{i}-\mathbf{r}_{j}\right|}}_{\begin{array}{c}
\text { Termo de } \\
\text { interacáo eletrostática } \\
\text { elétron- létron }
\end{array}} \underbrace{\sum_{A=1}^{M} \sum_{B>A}^{M} \frac{Z_{A} Z_{B}}{\left|\mathbf{R}_{A}-\mathbf{R}_{B}\right|}}_{\begin{array}{c}
\text { Termo de } \\
\text { interaçáo eletrostática } \\
\text { núcleo-núcleo }
\end{array}}
\end{aligned}
$$

Em geral, o desenvolvimento exato da Eq. 3.1 não é factível, tornando-se necessária a utilização de abordagens aproximativas. A primeira, chamada de separação de BornOppenheimer [122] (ou adiabática), considera os núcleos fixos face ao rápido movimento eletrônico ( $-\sum_{A=1}^{M} \frac{1}{2 M_{A}} \nabla_{A}^{2}=\epsilon_{0}$, onde $\epsilon_{0}$ é uma constante com dimensão de energia). Essa aproximação permite desacoplar a equação de Schrödinger independente do tempo em equações específicas para os movimentos nuclear e eletrônico, sendo este último governado pelo potencial efetivo obtido a partir da solução do Hamiltoniano $\mathcal{H}_{\text {ele }}$, associado ao deslocamento dos elétrons

$$
\mathcal{H}_{\text {ele }} \Psi_{\text {ele }}(\mathbf{r}, \mathbf{R})=E_{\text {ele }}(\mathbf{R}) \Psi_{\text {ele }}(\mathbf{r}, \mathbf{R})
$$

na qual

$$
\mathcal{H}_{\text {ele }} \equiv-\sum_{i=1}^{N} \frac{1}{2} \nabla_{i}^{2}-\sum_{i=1}^{N} \sum_{A=1}^{M} \frac{Z_{A}}{\left|\mathbf{r}_{i}-\mathbf{R}_{A}\right|}+\sum_{i=1}^{N} \sum_{j>i}^{N} \frac{1}{\left|\mathbf{r}_{i}-\mathbf{r}_{j}\right|}=\hat{T}+\hat{V}+\hat{U}
$$

é definido a menos de uma constante (em u.a.).

Grande parte do desenvolvimento na área de cálculos de estrutura eletrônica se deve ao advento da Teoria do Funcional da Densidade (DFT: Density Functional Theory), introduzida a partir dos teoremas propostos por Hohenberg e Kohn (HK) [123], em 1964. Em termos gerais, os teoremas de HK estabelecem que, dada a densidade eletrônica $n_{0}(\mathbf{r})$, exata do estado fundamental para um sistema com $N$ elétrons, em princípio é possível obter a função de onda correspondente $\Psi_{0}\left(\mathbf{r}_{1}, \mathbf{r}_{2}, \ldots, \mathbf{r}_{N}\right)$ também do estado fundamental

${ }^{1} \hbar=2 m=\frac{e^{2}}{2}=1$. As unidades do Sistema Internacional (SI) são inconvenientes para tratar das escalas atômicas, uma vez que podem surgir problemas de truncamentos quando os cálculos computacionais lidam com números demasiadamente pequenos. 
deste sistema. Isto significa que $\Psi_{0}$ é um funcional único de $n_{0}\left(\Psi_{0} \Leftrightarrow n_{0}\right)$, e todos observáveis físicos deste estado não-excitado são, da mesma forma, dependentes de $n_{0}$.

Assim, para um potencial $V_{\text {ext }}$ externo ao sistema eletrônico, o funcional de energia total $\left(E\left[n_{0}\right]\right)$ pode ser escrito como uma soma dos funcionais cinético $(\hat{T})$ e potencial $(\hat{U}+\hat{V})$ :

$$
\begin{aligned}
E\left[n_{0}\right]=\hat{T}\left[n_{0}\right]+\hat{U}\left[n_{0}\right]+\hat{V}\left[n_{0}\right]=\hat{T}\left[n_{0}\right] & +\hat{U}\left[n_{0}\right]+\int V_{\text {ext }}(\mathbf{r}) n_{0}(\mathbf{r}) d \mathbf{r} \\
& =\hat{F}\left[n_{0}\right]+\int V_{\text {ext }}(\mathbf{r}) n_{0}(\mathbf{r}) d \mathbf{r}
\end{aligned}
$$

Um importante avanço na aplicabilidade da DFT baseou-se na ideia de W. Kohn e L. Sham (KS) [124], em 1965, de empregar um sistema de referência de elétrons nãointeragentes cujo Hamiltoniano é conduzido por um potencial local efetivo $V_{\text {eff }}(\mathbf{r})$, que inclui os efeitos do sistema de muitos corpos. Explicitamente, a estratégia de Kohn-Sham (KS) consiste em resolver a seguinte equação de Schrödinger monoeletrônica ${ }^{1}$ (em u.a.) [125]:

$$
\left[-\nabla^{2}+V_{\text {eff }}(\mathbf{r})\right] \psi_{j}(\mathbf{r})=E_{j} \psi_{j}(\mathbf{r})
$$

para uma dada autofunção $\psi_{j}(\mathbf{r})$ (orbital). É frequente na literatura a definição do Hamiltoniano de $\mathrm{KS}\left(\mathcal{H}_{K S}\right)$ com base na equação acima [126]:

$$
\mathcal{H}_{K S}=\left[-\nabla^{2}+V_{\text {eff }}(\mathbf{r})\right]
$$

Assim, a Eq. 3.6 reduz o problema inicial de múltiplas partículas a um formalismo de partícula única, capaz de descrever as propriedades do sistema multieletrônico investigado. A solução é, então, atingida por meio de um processo autoconsistente - denominado esquema de KS -, descrito na seção 3.1.1, a seguir. Uma das contribuições que compõem $V_{\text {eff }}(\mathbf{r})$ é o potencial $V_{x c}(\mathbf{r})$, definido a partir da derivada do funcional de troca e correlação $\left(E_{x c}\left[n_{0}\right]\right): V_{x c}(\mathbf{r})=\frac{\delta E_{x c}\left[n_{0}\right]}{\delta n_{0}}$.

\subsubsection{Esquema de Kohn-Sham}

O esquema de KS resolve a Eq. 3.6 por meio de um processo autoconsistente que leva à densidade eletrônica do estado fundamental para um gás de elétrons interagentes $\left(n_{0}\right)$ a partir da solução do problema de referência não-interagente.

\footnotetext{
${ }^{1}$ Este tipo de equação também é comumente referido pela literatura de tipo-Schrödinger.
} 


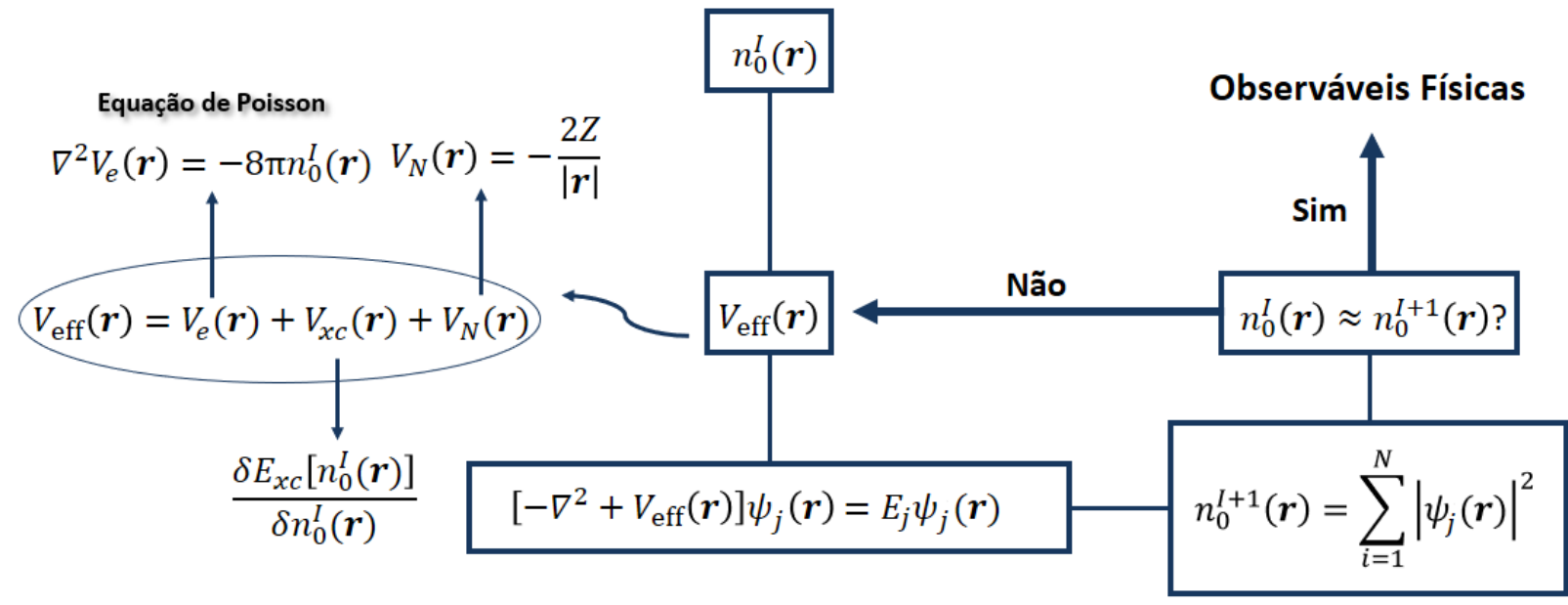

Figura 3.1: Ciclo de autoconsistência usual para o esquema de KS.

A Fig. 3.1 reproduz o ciclo de autoconsistência usual. Definida uma densidade inicial de carga $n_{0}^{I}(\mathbf{r})$, pode-se construir o potencial efetivo de Kohn-Sham $\left(V_{\text {eff }}(\mathbf{r})\right)$ pela soma das contribuições eletrônica $\left(V_{\mathrm{e}}(\mathbf{r})\right)$, de troca-correlação $\left(V_{x c}(\mathbf{r})\right)$, e relativa ao núcleo do átomo considerado $\left(V_{N}(\mathbf{r})\right)$, ou seja:

$$
V_{\text {eff }}(\mathbf{r}) \equiv V_{\text {eff }}\left[n_{0}^{I}\right]=V_{\mathrm{e}}\left[n_{0}^{I}\right]+V_{x c}\left[n_{0}^{I}\right]+V_{N}(\mathbf{r})
$$

onde $V_{\mathrm{e}}\left[n_{0}^{I}\right]$ é obtida como solução da equação de Poisson $\nabla^{2} V_{\text {eff }}\left[n_{0}^{I}\right]=-8 \pi n_{0}^{I}$ (r) (u.a.), $V_{x c}\left[n_{0}^{I}\right]$ depende do funcional de troca-correlação adequadamente escolhido, e $V_{N}(\mathbf{r})=-\frac{2 Z}{|\mathbf{r}|}$ (sendo $Z$ o número atômico). Com o orbital $\psi_{j}(\mathbf{r})$ previamente estabelecido, e com $V_{\text {eff }}(\mathbf{r})$ obtido pela Eq. 3.8 para a densidade $n_{0}^{I} \equiv n_{0}^{I}(\mathbf{r})$, torna-se, então, factível resolver a Eq. 3.6. Este procedimento gera uma nova densidade de carga $n_{0}^{I+1}$, baseada no somatório das funções $\left|\psi_{j}(\mathbf{r})\right|^{2}$ sobre os estados ocupados, isto é

$$
n_{0}^{I+1}(\mathbf{r})=\sum_{j}\left|\psi_{j}(\mathbf{r})\right|^{2}
$$

O processo iterativo segue até que $n_{0}^{I}$ (entrada) e $n_{0}^{I+1}$ (saída) difiram apenas de uma quantidade pré-estabelecida (critério de convergência), resultando na densidade eletrônica do estado fundamental do sistema. Por outro lado, caso esse critério não seja satisfeito, o ciclo autoconsistente continua com a construção de um novo potencial efetivo de KS a partir de $n_{0}^{I+1}$, ou seja, $V_{\text {eff }}\left[n_{0}^{I+1}\right]$. 


\subsubsection{Escolha do funcional de troca-correlação $\left(E_{x c}\right)$}

Dentre as abordagens disponíveis para resolver as equações de KS, uma possibilidade é tratar o potencial de troca-correlação segundo a denominada Aproximação da Densidade Local (LDA: Local Density Approximation), introduzida por Kohn e Sham na referência [124]. Trata-se da forma mais simples e historicamente relevante de $E_{x c}[n]$, a qual supõe que um sistema real não-homogêneo pode ser dividido em volumes infinitesimais com a mesma densidade eletrônica local $n(\mathbf{r})$, e reduz o sistema a um gás homogêneo de elétrons interagentes. Nesta aproximação, os termos de troca $\left(E_{x}^{\mathrm{LDA}}[n]\right)$ e correlação $\left(E_{c}^{\mathrm{LDA}}[n]\right)$ são obtidos separadamente:

$$
E_{x c}^{\mathrm{LDA}}[n]=E_{x}^{\mathrm{LDA}}[n]+E_{c}^{\mathrm{LDA}}[n]
$$

Embora se conheça a expressão exata para a energia de troca (ou exchange), em u.a.

$$
E_{x}^{\mathrm{LDA}}[n]=-\frac{3}{4} \sqrt[3]{\left(\frac{3}{\pi}\right)} \int n(\mathbf{r})^{4 / 3} d \mathbf{r}
$$

a energia de correlação é complexa e não pode ser determinada de modo analítico, nem mesmo para o caso do gás homogêneo de elétrons. Assim, o termo $E_{c}^{\mathrm{LDA}}[n]$ é parametrizado, e as expressões aproximadas podem ser obtidas aplicando-se teoria de perturbação [127, 128]. A LDA é uma aproximação bem sucedida para diversos sistemas de interesse, mesmo quando estes se desviam significativamente do conceito do gás homogêneo de elétrons, referência para a construção deste funcional. Uma das razões para este sucesso é a sua natureza sistemática de cancelamento de erros: enquanto, em geral, $E_{c}^{\mathrm{LDA}}[n]$ é subestimado, $E_{x}^{\mathrm{LDA}}[n]$ é superestimado, revertendo-se em bons resultados para $E_{x c}^{\mathrm{LDA}}[n]$. Isto não ocorre por acaso: para qualquer densidade $n \equiv n(\mathbf{r})$, deve-se satisfazer o vínculo $\int n_{x c}^{\mathrm{LDA}}\left(\mathbf{r}, \mathbf{r}^{\prime}\right) d \mathbf{r}^{\prime}=-1$ [129], exclusivamente possível se os erros integrados de $n_{x}^{\mathrm{LDA}}$ forem cancelados pelos erros integrados de $n_{c}^{\mathrm{LDA}}$.

Quando o sistema apresenta polarização de $\operatorname{spin}\left(n^{\uparrow}(\mathbf{r}) \neq n^{\downarrow}(\mathbf{r})\right)$, é conveniente utilizar a Aproximação da Densidade Local por Spin (LSDA: Local Spin Density A pproximation), para a qual uma das parametrizações disponíveis foi proposta por von Barth e Hedin [127], em 1972 - e que será utilizada neste trabalho como funcional de troca-correlação. Nela, a densidade eletrônica $n(\mathbf{r})$ é substituída por uma matriz densidade generalizada $\rho(\mathbf{r})$ que contém a informação a respeito dos spins dos elétrons, pois leva em conta a densidade de 
magnetização $\mathbf{m}(\mathbf{r})$ :

$$
\rho(\mathbf{r})=\frac{n(\mathbf{r})}{2} \mathbb{1}_{2 \times 2}+\frac{\mathbf{m}(\mathbf{r})}{2} \cdot \boldsymbol{\sigma}
$$

onde $\mathbb{1}_{2 \times 2}$ (ou simplesmente $\mathbb{1}$ ) simboliza a matriz unitária $2 \times 2$, e $\boldsymbol{\sigma}=\left(\sigma_{x}, \sigma_{y}, \sigma_{z}\right)$ são as matrizes de Pauli. Logo, o potencial efetivo de KS pode ser decomposto em duas partes: uma magnética, b, e outra não-magnética, $V_{\mathrm{NM}}$. O Hamiltoniano de KS (Eq. 3.7) é, então, reescrito no âmbito da LSDA da seguinte maneira [76]:

$$
\mathcal{H}_{K S}^{\mathrm{LSDA}}=\left[-\nabla^{2}+V_{\mathrm{NM}}(\mathbf{r})\right] \mathbb{1}+\mathbf{b} \cdot \boldsymbol{\sigma}
$$

\subsection{Formalismo LMTO-ASA no espaço direto (RS- LMTO-ASA)}

\subsubsection{Preâmbulo}

Além da escolha do funcional, a definição do método é de extrema importância para a simulação teórica de materiais, e deve ser pautada tanto nas propriedades que se deseja investigar quanto nas características organizacionais intrínsecas ao sistema, como a existência ou não de quebra de simetria de inversão. Isto porque a determinação do método implica diretamente na forma como são descritas as funções $\psi_{j}(\mathbf{r})$ nas equações de KS, sejam elas expandidas em uma base de ondas planas, por exemplo, ou em orbitais do tipo atômico. No presente trabalho, a investigação das propriedades eletrônicas e magnéticas das bicamadas de $\mathrm{Pd} / \mathrm{Fe} / \operatorname{Ir}(111)$ e $\mathrm{Pd} / \mathrm{Co} / \operatorname{Ir}(111)$ é desenvolvida com base no método linear de Andersen [130, 131], o Linear Muffin-Tin Orbital (LMTO). Nele, o potencial considerado é do tipo "muffin-tin", no qual, fora da região intersticial (constante), é descrito por uma função esfericamente simétrica; o sólido é, portanto, construído a partir da sobreposição de esferas centradas nos seus vários sítios. Já os autoestados da equação de KS (Eq. 3.6) são expandidos em torno de uma base de funções $\left\{\chi_{R L, i}\right\}$ independente da energia, construída a partir da combinação linear de soluções da equação tipo-Schrödinger e suas primeiras derivadas dentro das esferas muffin-tin para uma determinada energia $E_{\nu}$. Assim, recai-se em um problema de autovalores cujos autoestados podem ser bem descritos por poucas funções de base que apresentam estas características.

O método LMTO na Atomic Sphere Approximation (LMTO-ASA), em qualquer uma das bases apresentadas na seção B.1, permite resolver o problema de autovalores (Eq. B.2) no espaço recíproco sem dificuldade. Este esquema é mais apropriado para sistemas 
nos quais a simetria de inversão não é quebrada (ou seja, com periodicidade translacional). No entanto, as estruturas de interesse nesta dissertação são cadeias atômicas e aglomerados compactos finitos depositados em uma superfície, onde, pela quebra de simetria, é difícil a solução no espaço k. Assim, para a obtenção da estrutura eletrônica e propriedades magnéticas dos sistemas propostos, a utilização do método de primeiros princípios no espaço real (RS: Real Space) RS-LMTO-ASA [132-134] é bastante conveniente. Diferentemente do formalismo LMTO-ASA no espaço recíproco, no qual é fundamentado, o RS-LMTO-ASA calcula os autovalores da Eq. B.2 usando o Método de Recorrência de Haydock [135] em conjunto com o terminador de Beer-Pettifor [136] (seção B.2). Conforme apresentado pela Eq. B.2, o problema de autovalores é escrito como [137-140]:

$$
\left(\mathcal{H}-E_{\nu}\right) \mathbf{u}=\mathbb{O}
$$

e a função de onda $\Psi_{E}$ correspondente é dada por:

$$
\Psi_{E}=\sum_{R L}\left[\varphi_{R l}(r)+\left(E-E_{\nu}\right) \dot{\varphi}_{R l}(r)\right] Y_{L}(\hat{\mathbf{r}}) u_{R L}(E)
$$

Na Eq. 3.15, $\varphi_{R l}(r)$ e $\dot{\varphi}_{R l}(r)$ são, respectivamente, as soluções da equação tipoSchrödinger radial para uma única partícula, e sua primeira derivada em relação à energia, definidas na seção B.1.4.

A Hamiltoniana na base ortogonal pode ser expressa em termos dos assim chamados parâmetros tight-binding (TB) como se segue:

$$
\mathcal{H}=\overline{\mathbf{h}}(\mathbb{1}+\overline{\mathbf{o}} \overline{\mathbf{h}})^{-1}+E_{\nu} \sim E_{\nu}+\overline{\mathbf{h}}(\mathbb{1}-\overline{\mathbf{o}} \overline{\mathbf{h}}+\overline{\mathbf{o}} \overline{\mathbf{h}} \overline{\mathbf{h}}+\ldots) \Rightarrow \mathcal{H}^{(1)}=E_{\nu}+\overline{\mathbf{h}}
$$

Então, na aproximação de primeira ordem em $\overline{\mathbf{h}}$ e desprezando o termo $\overline{\mathbf{h}} \overline{\mathbf{h}}$ [proporcional a $\left.\left(E-E_{\nu}\right)^{2}\right]$, a Hamiltoniana $\mathcal{H}^{(1)}$ fica:

$$
\mathcal{H}^{(1)}=\overline{\mathbf{C}}+\bar{\Delta}^{1 / 2} \overline{\mathbf{S}} \bar{\Delta}^{1 / 2}
$$

Aqui, $\overline{\mathbf{C}}, \overline{\boldsymbol{\Delta}}^{1 / 2}$ são parâmetros de potencial e $\overline{\mathbf{S}}$ é a matriz de estrutura na base mais localizada (relativa às posições atômicas). Uma boa descrição das partes ocupadas das bandas $s, p$ e $d$ é atingida considerando-se apenas a Hamiltoniana de primeira ordem (Eq. B.87). Em geral, as mudanças na estrutura eletrônica causadas pela adição do termo de segunda ordem são pequenas [141], mas sua inclusão torna o processo autoconsistente muito mais custoso computacionalmente. 
Nesta seção, o método LMTO de espaço direto na aproximação da esfera atômica (ASA: Atomic Sphere Approximation) - o RS-LMTO-ASA [132-134] - será brevemente apresentado. Sua implementação computacional foi desenvolvida inicialmente na década de 80 pelo grupo da Prof ${ }^{a}$. Dr ${ }^{a}$. Sonia Frota-Pessôa, do Instituto de Física da Universidade de São Paulo, e tem sido desde então aperfeiçoada por outros grupos, em particular o da $\operatorname{Prof}^{a}$. Dr ${ }^{a}$. Ângela Burlamaqui Klautau (UFPA - Belém/PA) e o do Prof. Dr. Olle Eriksson (Universidade Uppsala - Suécia). Durante toda a descrição da metodologia serão apenas tratados os estados eletrônicos, imersos em um potencial associado aos núcleos (fixos) do material estudado, incluídos em $V_{\text {eff }}(\mathbf{r})$. Além disso, considerando o RS-LMTOASA uma abordagem que parte do formalismo de KS, o índice $K S$ do Hamiltoniano será ocultado nas equações a seguir (i.e. $\mathcal{H}_{K S}^{\mathrm{LSDA}} \equiv \mathcal{H}$ ).

\subsubsection{Implementação do RS-LMTO-ASA}

A implementação computacional do método de espaço direto RS-LMTO-ASA constituise de dois processos autoconsistentes entrelaçados, a parte geral e a parte atômica, respectivamente esquematizados pelas Figs. 3.2 e 3.3. Em termos gerais, a parte atômica encarrega-se da determinação do potencial e dos parâmetros $\left(C_{R l}, \Delta_{R l}, Q_{R l}\right)$ para cada sítio $R$ não-equivalente considerado no cálculo. Nesse sentido, define-se a equivalência entre dois sítios se os parâmetros de potencial associados a cada um deles são os mesmos, levando à ocupações eletrônicas e densidades locais de estados idênticos. Por outro lado, na parte geral, o problema de autovalores (Eq. 3.14) é resolvido por completo, utilizandose os parâmetros na base ortogonal encontrados ao longo da execução da parte atômica; com isso é obtida a estrutura eletrônica do sistema. A divisão do problema em duas partes é viável devido ao fato de os valores do parâmetro de mistura $\overline{\mathbf{Q}}$ serem constantes e independentes do potencial. Sendo assim, nas subseções a seguir, serão apresentados em mais detalhes ambos o processos que constituem o RS-LMTO-ASA. 


\section{Parte geral}

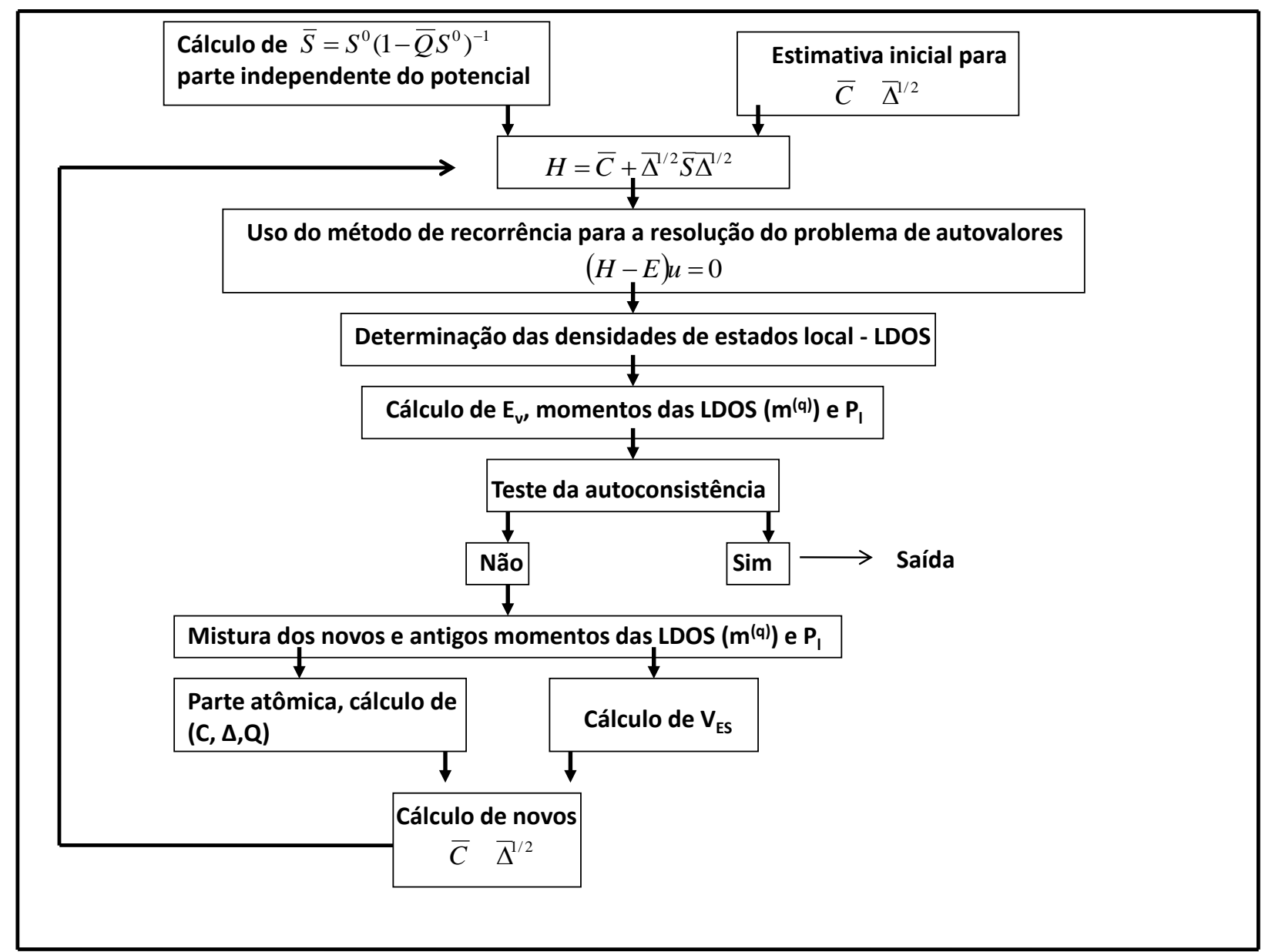

Figura 3.2: Fluxograma representativo da parte geral do método RS-LMTO-ASA [76].

Este processo inicia-se com duas etapas independentes: $(i)$ o cálculo da matriz de estrutura $\overline{\mathbf{S}}$, que depende apenas da organização espacial do material; e ( $i i)$ a obtenção dos parâmetros de potencial $\overline{\mathbf{C}}$ e $\bar{\Delta}^{1 / 2}$. A segunda etapa consiste na conversão dos parâmetros $\mathbf{C}, \boldsymbol{\Delta}^{1 / 2}$, e $\mathbf{Q}$, determinados na representação ortogonal durante a parte atômica, para a base TB, mediante a seguinte relação [131]:

$$
\frac{\Delta_{R l}^{1 / 2}}{\bar{\Delta}_{R l}^{1 / 2}}=1-\left(\bar{Q}_{R l}-Q_{R l}\right) \frac{\bar{C}_{R l}-E_{\nu}}{\bar{\Delta}_{R l}}=\frac{C_{R l}-E_{\nu}}{\bar{C}_{R l}-E_{\nu}}
$$

Esta expressão pode ser facilmente derivada das Eqs. B.89, B.90, B.76, e B.77, e sua dedução foi desenvolvida em detalhes na referência [79]. Note que, na Eq. 3.18, utiliza-se a notação sem barra estabelecida na seção B.1.7 para a base ortogonal. O parâmetro $\overline{\mathbf{Q}}$, independente da estrutura do material, relaciona a matriz $\mathbf{S}$ escrita na base ortogonal 
com a da base TB, por meio da expressão:

$$
\overline{\mathbf{S}}=\mathbf{S}(\mathbb{1}-\overline{\mathbf{Q}} \mathbf{S})^{-1}
$$

Os valores de $\mathbf{S}$ e $\mathbf{\mathbf { Q }}$ estão tabelados na literatura [130] e foram apresentados na seção B.1.6. Como a estrutura permanece invariante durante todo o processo autoconsistente, o parâmetro $\overline{\mathbf{S}}$, que possui dimensão $9 N \times 9 N$ (sendo $N$ o número de constituintes no sistema cristalino, geralmente da ordem de $10^{3}$ ), é obtida uma única vez. Entretanto, devido ao caráter local de $\overline{\mathbf{S}}$ pode-se considerar um aglomerado menor em torno de cada sítio, com cerca de 20 átomos [79], reduzindo o problema à inversão de uma matriz de dimensão $180 \times 180$. Calculada a constante de estrutura do material com o auxílio da Eq. 3.19, concomitantemente se faz uma estimativa inicial dos elementos de matriz $\bar{C}_{R l}$ e $\bar{\Delta}_{R l}$ em cada esfera não-equivalente, obtidas a partir de cálculos da parte atômica para os momentos $m_{R l}^{(q)}$ da LDOS (de ordem $q$ ) e do parâmetro $P_{l}$ apropriados; os valores $\bar{C}_{R l}$ e $\bar{\Delta}_{R l}^{1 / 2}$ se modificam a cada iteração. De posse destas quantidades, é possível construir a Hamiltoniana $\mathcal{H}^{(1)}$ na aproximação de primeira ordem em $\left(E-E_{\nu}\right)$, dada pela Eq. 3.17. Então, a partir do Método de Recorrência (seção B.2) são obtidos os novos autovalores e os momentos $m_{R l}^{(q)}$, a saber:

$$
m_{R l}^{(q)}=\int_{-\infty}^{E_{F}}\left(E-E_{\nu, R l}\right)^{q} N_{R L}(E) d E
$$

onde $q$ varia entre 0,1 e 2 e $m_{R l}^{(q)} \equiv m^{(q)}$ são relativos à energia escolhida $E_{\nu, R l} \equiv E_{\nu}$. Por definição, o momento de ordem zero $\left(m^{(0)}\right)$ representa a ocupação de cada orbital $s, p$ e $d$. Por sua vez, assumindo o valor de $E_{\nu}$ como a energia do centro de gravidade da banda ocupada, o momento de primeira ordem $\left(m^{(1)}\right)$ é, por construção, nulo. Na parte geral, também são obtidas as condições de contorno para cada esfera não-equivalente, definidas por meio dos parâmetros $P_{l}[133,138]$ :

$$
\begin{array}{r}
P_{l}=\frac{1}{2}-\frac{1}{\pi} \arctan \left(D_{\nu}\right)= \\
=\frac{1}{2}-\frac{1}{\pi} \arctan \left\{1+(2 l+1)\left[\frac{Q_{l}^{-1}}{2(2 l+1)} \frac{C_{l}-E_{\nu}}{C_{l}-E_{\nu}-\Delta Q_{l}^{-1}}-1\right]\right\}
\end{array}
$$

onde $0<P_{l} \leq 1$ e $D_{\nu}$ é a derivada logarítmica da solução da equação de KS impondo condições de continuidade no contorno da esfera [137]. Obtidos os momentos da LDOS $\left(m^{(0)}, m^{(1)}=0, m^{(2)}\right)$, o parâmetro $P_{l}$, e de posse da energia $E_{\nu}$, a parte atômica (subseção a seguir) é, então, calculada. A parte geral alcança a convergência quando não existirem variações significativas entre os valores novos e antigos dos momentos da LDOS e de $P_{l}$ 
(veja a Fig. 3.2).

\section{Parte atômica}

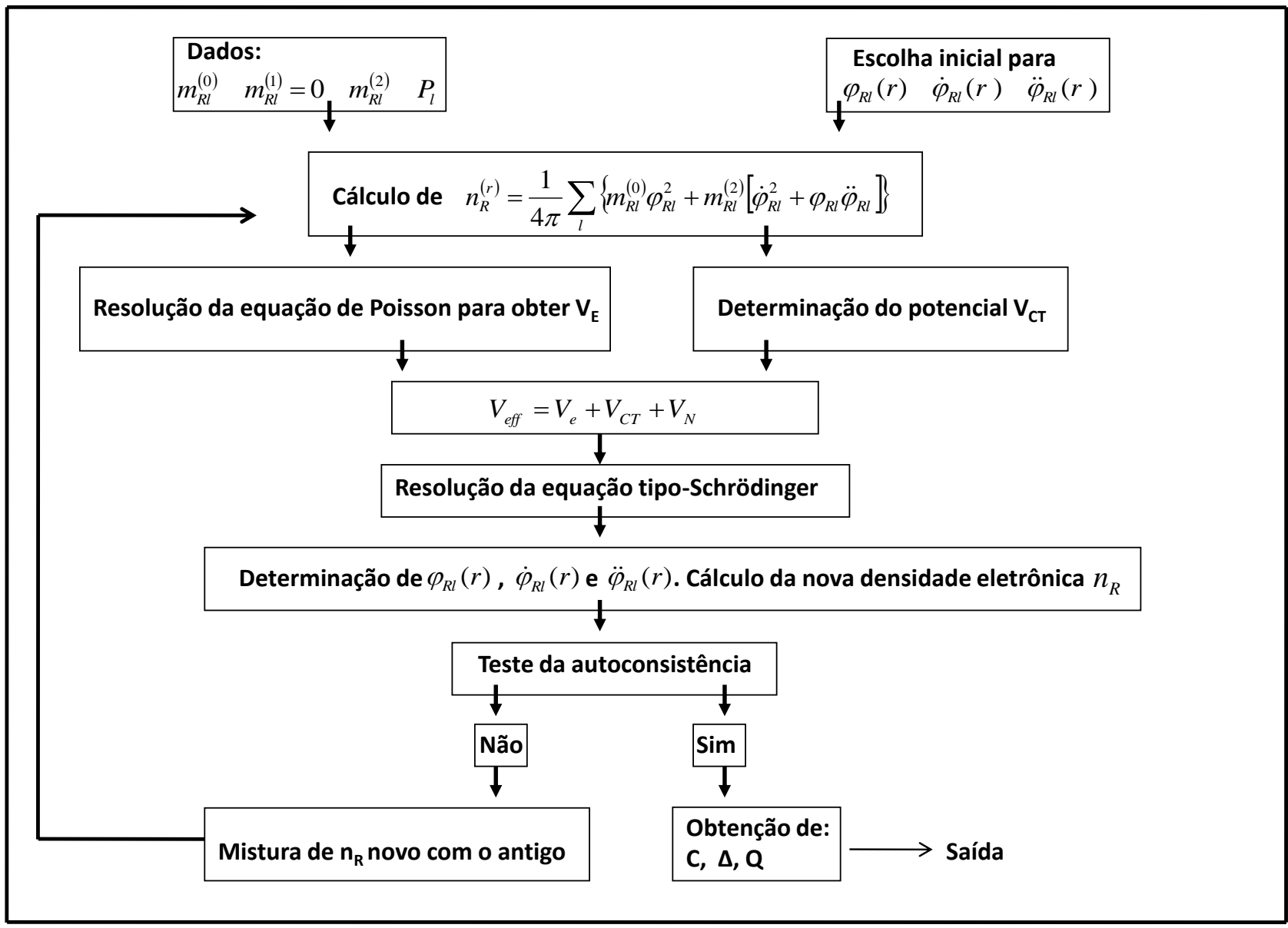

Figura 3.3: Fluxograma representativo da parte atômica do método RS-LMTO-ASA [76].

Na parte atômica, esquematizada pela Fig. 3.3, são obtidos os parâmetros de potencial em cada esfera não-equivalente, posteriormente utilizados no esquema da parte geral. Inicialmente é feito o cálculo da média esférica da densidade eletrônica para cada sítio $R$ considerado no cristal ou aglomerado, usando [131] $\left(m^{(1)}=0\right)$ :

$$
n(\mathbf{r})=\frac{1}{4 \pi} \sum_{l}\left[m_{R l}^{(0)} \varphi_{R l}^{2}+m_{R l}^{(2)}\left(\dot{\varphi}_{R l}+\varphi_{R l} \ddot{\varphi}_{R l}\right)\right]
$$

onde $m_{R l}^{(q)}$ são os momentos definidos pela Eq. 3.20, e os $\varphi_{R l}$ são as soluções das equações tipo-Schrödinger na região em que $|\mathbf{r}| \leq s$. A Eq. 3.22 é resolvida a partir de uma 
estimativa inicial para $\varphi_{R l}$ e usando valores fixados de $m_{R l}^{(0)}$ e $m_{R l}^{(2)}$, obtidos em cada iteração da parte geral.

Com a densidade eletrônica calculada, o potencial eletrostático $\left(V_{e}\right)$ também pode ser determinado através da solução da equação de Poisson. A este potencial é adicionado o termo de troca e correlação $\left(V_{X C}\right)$ (obtido através da LSDA) e também a contribuição nuclear $\left(V_{N}\right)$ do átomo em questão (com $Z$ prótons no núcleo) dado simplesmente por $V_{N}(r)=-\frac{2 Z}{r}$. Logo, o potencial total (ou efetivo) $V_{\text {eff }}$ na esfera em $\mathbf{R}$ constitui-se da soma:

$$
V_{\mathrm{eff}}=V_{e}+V_{X C}+V_{N}=V_{e}+V_{C T}[n(\mathbf{r})]+V_{N}[n(\mathbf{r})]
$$

Com o valor de $V_{\text {eff }}$ e as condições de contorno expressas em termos do parâmetro $P_{l}$ (Eq. 3.21) fixado no início do processo, resolve-se a equação tipo-Schrödinger radial a fim de se obter os novos orbitais calculados em torno da energia $E_{\nu}$. Neste ponto da autoconsistência, verifica-se se a diferença entre o novo e o antigo valor da densidade eletrônica é menor que uma quantidade pré-estabelecida (critério de convergência). A comparação positiva encerra a parte atômica, e as funções $\varphi_{R l}, \dot{\varphi}_{R l}$, e $P_{l}$ ficam determinadas. Enquanto esta diferença for maior que o critério escolhido, o processo continua com a mistura entre os dois valores de $n(\mathbf{r})$, conduzida pelo fator $\alpha(0 \leq \alpha \leq 1)$ por meio da seguinte substituição:

$$
n(\mathbf{r}) \rightarrow \alpha n_{\text {novo }}(\mathbf{r})+(1-\alpha) n_{\text {antigo }}(\mathbf{r})
$$

Tem-se, assim, uma nova estimativa para a densidade eletrônica. Atingida a convergência, encontram-se, então, os novos parâmetros de potencial na base ortogonal $\left(C_{R l}, \Delta_{R l}, Q_{R l}\right)$ para cada sítio não-equivalente do sistema. $C_{R l}$ e $\Delta_{R l}$ estão relacionados, respectivamente, ao centro e à largura da banda, enquanto $Q_{R l}$ é calculado de tal forma que $\mathbf{o} \equiv \mathbb{O}$, mencionados nas seções precedentes. Entretanto, cabe lembrar que, no Método de Recorrência (seção B.2), é conveniente empregar estes parâmetros em uma base mais localizada. Portanto, com o auxílio da Eq. 3.18, obtêm-se facilmente os mesmos parâmetros na base TB $\left(\bar{C}_{R l}, \bar{\Delta}_{R l}, \bar{Q}_{R l}\right)$, passo que é realizado no decorrer da parte geral.

Paralelamente a este processo, é calculado o potencial de Madelung $\left(V_{E S}\right)$, que corrige os efeitos provocados pela existência de carga nas esferas vizinhas e a contribuição eletrônica da esfera situada no próprio sítio. Isto porque, durante a execução da parte atômica, considera-se $V_{R}$ nulo na superfície de cada esfera em $\mathbf{R}$. Ou seja, de fato deslocam-se a energia escolhida $E_{\nu, R l}$ e os parâmetros $C_{R l}$ por uma quantidade expressa 
pelo termo eletrostático:

$$
V_{E S, i}=\sum_{j \neq i} \frac{2 \Delta Q_{j}}{\left|\mathbf{R}_{i}-\mathbf{R}_{j}\right|}+\frac{2 \Delta Q_{i}}{s}
$$

para sítios genéricos $j$ vizinhos do sítio $i$ de referência. Na Eq. 3.25, pela notação usual, $\left|\mathbf{R}_{i}-\mathbf{R}_{j}\right|$ é a distância entre as posições $i$ e $j, s$ é o raio de Wigner-Seitz do átomo, e $\Delta Q_{i}$ representa a transferência de carga encontrada no sítio $i$, cuja obtenção será detalhada nas próximas seções para o caso de perturbações locais.

\subsubsection{Processo autoconsistente para sistemas bidimensionais (su- perfícies) no RS-LMTO-ASA}

Conforme mencionado, o esquema do processo autoconsistente do RS-LMTO-ASA é o mesmo para qualquer sistema metálico que se deseja investigar, podendo ser aplicado para estruturas cristalinas [132-134], impurezas e defeitos locais em hospedeiros metálicos [139, 142], e outros sistemas como superfícies limpas e com nanoestruturas depositadas $[34,39,140,143-145]$. Entretanto, o potencial eletrostático $\left(V_{E S}\right)$ e o nível de Fermi $\left(E_{F}\right)$ devem ser determinados de acordo com o problema estudado.

No caso das superfícies metálicas, a região que representa o vácuo não é preenchida por funções que descrevem a carga encontrada fora da região definida pelas esferas de WignerSeitz (WS) [146]. Portanto, um artifício de cálculo amplamente utilizado no formalismo LMTO-ASA é a inclusão de um número finito de camadas de esferas vazias acima da superfície metálica (em geral, uma ou duas). Estas esferas possuem carga de valência nula $\left(Q_{\text {valência }}=0\right)$ e o mesmo raio de WS do material hospedeiro. De modo equivalente aos demais átomos, os parâmetros de potencial das esferas vazias são determinados de maneira autoconsistente. A presença de elétrons fora da superfície determina que a camada de esferas vazias seja carregada negativamente, ao passo que, devido ao princípio de conservação de carga, a camada atômica é carregada positivamente. Portanto, o sistema comporta-se como um capacitor de placas paralelas, modificando o potencial eletrostático em sítios distantes da superfície e deslocando o nível de Fermi até que a densidade de estados acomode o número reduzido de elétrons no metal [147].

A fim de evitar que o nível de Fermi seja deslocado a cada iteração, redefine-se a escala de energia, de forma que o potencial seja nulo em sítios distantes da superfície, dentro do material. Para tal, subtrai-se o valor de $V_{E S}$ de todas as camadas pelo potencial eletrostático obtido para o bulk $\left(V_{b u l k}\right)$. Assim, para o cálculo da superfície limpa, 
multicamadas, ou outros sistemas com simetria bidimensional, o valor de $E_{F}$ é fixado naquele encontrado para o cálculo do bulk. Por definição, a energia de Fermi do material volumétrico é obtida utilizando a seguinte condição:

$$
\sum_{R L} \int_{-\infty}^{E_{F}} N_{R L}(E) d E=Q_{\text {valência }}
$$

onde $Q_{\text {valência }}$ é a carga de valência, e $N_{R L}(E) d E$ é a LDOS para cada orbital $L=(l, m)$ e cada sítio $R$ na faixa de energia entre $E$ e $E+d E$. Com o nível de Fermi fixado, é possível determinar a transferência de carga em cada sítio de maneira autoconsistente, inclusive para as esferas vazias.

Para sistemas cristalinos, o potencial é obtido pela soma de Ewald [148]. Este cálculo é mais simples em comparação à situação com falta de simetria de inversão, pois o número de átomos não-equivalentes é conhecido a priori, e depende apenas dos constituintes e de suas posições na célula primitiva do metal que se deseja calcular. Para o caso de uma superfície, por outro lado, as transferências de carga $\left(\Delta Q_{\text {sup }}\right)$ induzem potenciais eletrostáticos diferentes em todas as camadas - aumentando a quantidade e a complexidade dos sítios não equivalentes. Em 1991, H. L. Skriver et al. [147] introduziram alterações na soma de Ewald tridimensional, obtendo uma técnica para o caso bidimensional. O cálculo de superfícies utilizando o método RS-LMTO-ASA inclui as transferências de carga na expansão obtida por Skriver, resultando, assim, tanto no potencial de Madelung (Eq. 3.25) quanto no valor de $V_{E S}$ em cada sítio do sistema. Como o valor de $\Delta Q_{\text {sup }}$ torna-se desprezível para as camadas mais internas, o processo autoconsistente apenas leva em consideração os planos próximos às esferas vazias, em quantidade definida pelo usuário nos arquivos de input. Para os demais sítios, os parâmetros de potencial, $\bar{\Delta}^{1 / 2}$ e $\overline{\mathbf{C}}$, calculados do material bulk são empregados na construção da matriz Hamiltoniana.

No formalismo RS-LMTO-ASA, a estrutura do sistema metálico semi-infinito é simulada por um aglomerado contendo alguns milhares de átomos, arranjados em vários planos atômicos paralelos ao plano cristalográfico desejado. A princípio, calcula-se de forma autoconsistente uma camada do metal $[\mathrm{MT}(S)]$ justaposta a uma camada de esferas vazias $(\mathrm{ESF}-1)$. Partindo das estimativas iniciais para os momentos da densidade de estados $m_{R l}^{(0)}, m_{R l}^{(1)}=0, m_{R l}^{(2)}$, e $P_{l}$ associados aos átomos pertencentes à estes dois planos, obtém-se, seguindo o esquema para a parte atômica, os parâmetros $C_{R l}, \Delta_{R l}$, e $Q_{R l}$ de cada sítio na base ortogonal, os quais podem ser relacionados aos parâmetros $\bar{\Delta}^{1 / 2}$ e $\overline{\mathbf{C}}$ na base TB. A consideração de apenas duas camadas não equivalentes implica que os átomos contidos nos demais planos apresentam os mesmos parâmetros de potencial 
obtidos para o material bulk. Dessa forma, assumindo a aproximação em primeira ordem, calcula-se a Hamiltoniana $\mathcal{H}^{(1)}$, e, a partir desta, o valor de $P_{l}$, a LDOS, e os respectivos novos momentos $m_{R l}^{(q)}$, tanto para ESF-1 quanto para $\operatorname{MT}(S)$. Por outro lado, com as transferências de carga em $\operatorname{ESF}-1, \operatorname{MT}(S)$ e na camada metálica adjacente, $\operatorname{MT}(S-1)$, obtém-se os potenciais eletrostáticos $V_{E S}$ nos vários planos considerados no cálculo. A Fig. 3.4 mostra um exemplo de esquema de planos utilizados na convergência de uma superfície $f c c(111)$.

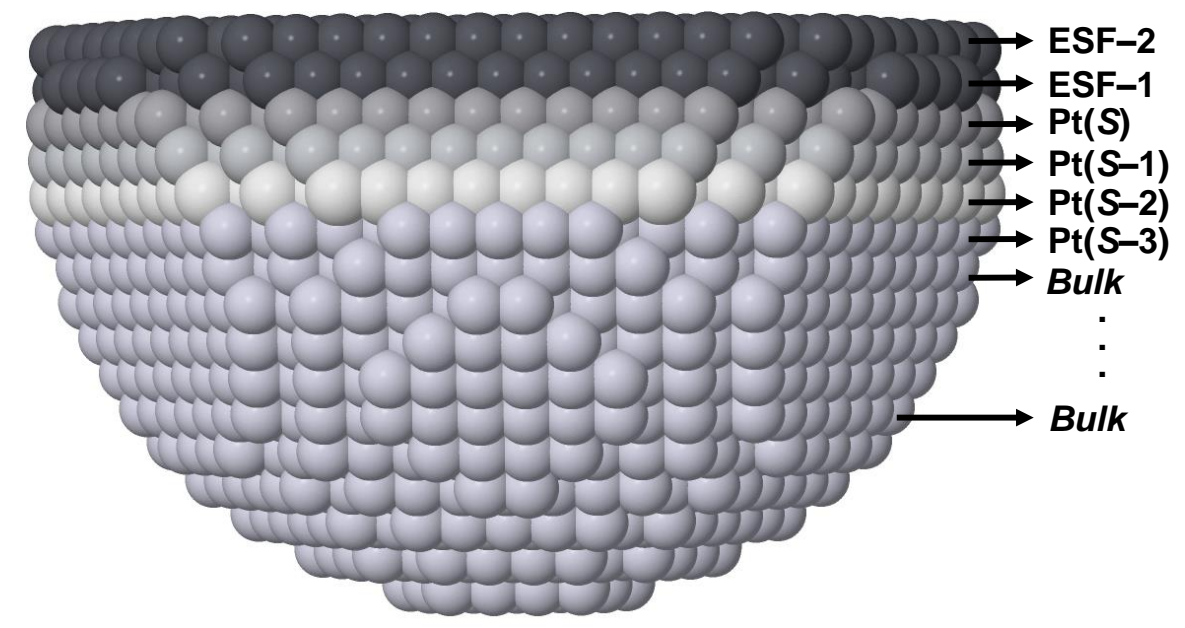

Figura 3.4: Representação esquemática de um cluster construído no espaço real com a identificação dos planos que constituem a superfície (de Pt) $f c c(111)$. Cada esfera retrata um átomo no cluster.

Obtida a autoconsistência, outra camada de esferas vazias (ESF-2) é incluída no cálculo. Dessa maneira, tornam-se três os átomos (ou sítios) não equivalentes considerados no processo: os pertencentes aos planos ESF-2, ESF-1 e $\mathrm{MT}(S)$. Os momentos $m_{R l}^{(q)}$ e o parâmetro $P_{l}$ de $\mathrm{ESF}-1$ e $\mathrm{MT}(S)$ são mantidos, enquanto, para ESF-2, estes valores partem novamente de estimativas iniciais ${ }^{1}$. Fixado e convergido o número de camadas de esferas vazias, resta a inclusão dos demais planos metálicos justapostos a $\operatorname{MT}(S)(\operatorname{MT}(S-1), \operatorname{MT}(S-2), \ldots)$, sucessivamente, até que os momentos $m_{R l}^{(q)}$ da LDOS e os parâmetros $P_{l}$ da última camada estejam de acordo com os obtidos para o material bulk. Esta conformidade envolve, essencialmente, a comparação efetiva entre as ocupações eletrônicas dos átomos da última camada e do bulk.

\footnotetext{
${ }^{1}$ Devido à sensibilidade na convergência camadas metálicas junto às esferas vazias, por serem estas artifícios de cálculo, é importante determinar pequenos valores para o parâmetro $\alpha$, que controla a mistura de $n(\mathbf{r})$, a fim de evitar uma elevada transferência de carga da superfície metálica (ou alta densidade eletrônica nas esferas vazias) - situação na qual dificilmente se atinge a autoconsistência.
} 


\subsubsection{Processo autoconsistente para defeitos em superfícies no RS-LMTO-ASA}

Após a execução do processo autoconsistente para o sistema bidimensional ideal (seção 3.2.3), os valores dos potenciais eletrostáticos, das transferências de carga e dos parâmetros de potencial são definidos para os átomos distantes do defeito introduzido na superfície, cuja presença não afeta significativamente as propriedades daqueles sítios. Considera-se que o nível de Fermi permanece inalterado, pois a inserção (ou adsorção) de defeitos na superfície metálica não muda de forma considerável o número de elétrons do sistema; toma-se, portanto, a energia de Fermi do material bulk. Finalmente, os parâmetros de corte $(L L)$ na recorrência são os mesmos utilizados na primeira etapa.

A presença de defeitos depositados ou impurezas embebidas em uma superfície perfeita causa perturbações locais, ou seja, em uma região determinada, tanto nas transferências de carga $\left(\Delta Q_{\text {local }}\right)$ como no potencial $\left(V_{E S_{\text {local }}}\right)$. Se $V_{E S_{\text {sup }}}$ e $\Delta Q_{\text {sup }}$ são os valores correspondentes obtidos para o sistema bidimensional sem defeitos, enquanto $V_{E S}$ e $\Delta Q$ são os parâmetros calculados para a superfície com impurezas introduzidas, estas quantidades estão relacionadas da seguinte maneira:

$$
\left\{\begin{array}{l}
\Delta Q=\Delta Q_{\text {sup }}+\Delta Q_{\text {local }} \\
V_{E S}=V_{E S_{\text {sup }}}+V_{E S_{\text {local }}}
\end{array}\right.
$$

posto que o princípio da superposição se aplica à energia potencial. Assim, suponha que para uma boa descrição do substrato livre foram necessárias duas camadas de esferas vazias (ESF-2 e ESF-1), quatro camadas metálicas não equivalentes ( $\mathrm{MT}(S), \operatorname{MT}(S-1)$, $\operatorname{MT}(S-2)$, e $\operatorname{MT}(S-3))$, e outras $n$ camadas com as características do material bulk. Considerando uma impureza substitucional sem relaxação da rede, por exemplo, a matriz constante de estrutura $\overline{\mathbf{S}}$ e o aglomerado representativo da superfície são os mesmos utilizados na primeira etapa. Por outro lado, caso se queira investigar um átomo adsorvido (adátomo) nesta superfície substitui-se, então, um único sítio aproximadamente central (no cluster) de ESF-1 pelo átomo do metal de transição desejado.

A construção da Hamiltoniana requer uma estimativa inicial para os parâmetros de potencial no sítio do defeito, ao passo que, para todos os outros átomos das diversas camadas metálicas, os valores de $\bar{\Delta}^{1 / 2}$ e $\overline{\mathbf{C}}$ são definidos naqueles obtidos para a superfície limpa. A partir do Método de Recorrência (seção B.2), calculam-se os valores de $N_{R L}(E)$, segundo os quais a transferência de carga total, $\Delta Q$, é determinada pela Eq. 3.26. Dessa forma, 
$\Delta Q_{\text {local }}$ é facilmente obtida pela Eq. 3.27, pois o valor de $\Delta Q_{\text {sup }}$ é conhecido a priori. O excesso de carga é distribuído entre os primeiros vizinhos em torno do defeito, obedecendo a lei da conservação das cargas no sistema. Por sua vez, o potencial eletrostático local, $V_{E S_{\text {local }}}$, pode ser determinado a partir da configuração de $\Delta Q_{\text {local }}$, e, usando a Eq. 3.27, encontra-se o valor de $V_{E S}$. De posse desta quantidade, calculam-se novos parâmetros de potencial, logo após submetidos à mistura com os valores antigos, para, então, reiniciar o esquema autoconsistente. O processo iterativo termina com a convergência nas ocupações - ou nos momentos da LDOS - dos(s) defeito(s).

Com o cálculo de primeiros princípios de sítio único devidamente realizado, convém incluir os primeiros vizinhos da impureza pertencentes à superfície no processo como átomos não equivalentes. Para os sítios vizinhos ao adátomo, inicialmente usam-se os parâmetros de potencial obtidos no caso da superfície livre, enquanto, para a própria impureza, os mesmos parâmetros são definidos naqueles encontrados pela etapa de sítio único como estimativa preliminar. De maneira análoga ao cálculo de sítio único, a carga excedente é, agora, distribuída entre os segundos vizinhos do adátomo. Com esta nova distribuição de carga, então, obtém-se os novos potenciais $V_{E S}$ relativos tanto à impureza quanto aos seus vizinhos mais próximos. Este processo dá origem a uma nova Hamiltoniana correspondente, cujos autovalores são determinados na parte geral do esquema autoconsistente (subseção 3.2.2), e assim sucessivamente, até que se atinja a convergência da impureza junto à primeira vizinhança.

A inclusão de vizinhos mais distantes termina quando o último conjunto considerado apresentar as mesmas ocupações - dentro de uma tolerância previamente definida - dos átomos metálicos na superfície livre. Nestes sítios, os valores de $\Delta Q_{\text {local }}$ e $V_{E S_{\text {local }}}$ devem ser desprezíveis, levando a $\Delta Q \sim \Delta Q_{\text {sup }}$ e $V_{E S} \sim V_{E S_{\text {sup }}}$ pela Eq. 3.27. A Fig. 3.5 apresenta de maneira ilustrativa o conjunto de primeiros e segundos vizinhos de um adátomo de $\mathrm{Fe}$ em uma superfície de Pt $f c c(111)$. Com frequência, a inclusão de primeiros e/ou segundos vizinhos é suficiente para que as perturbações locais induzidas pela presença da impureza sejam aceitavelmente pequenas. Embora aqui estejamos representando um adátomo, o processo é o mesmo para defeitos/impurezas embebidas na superfície. 


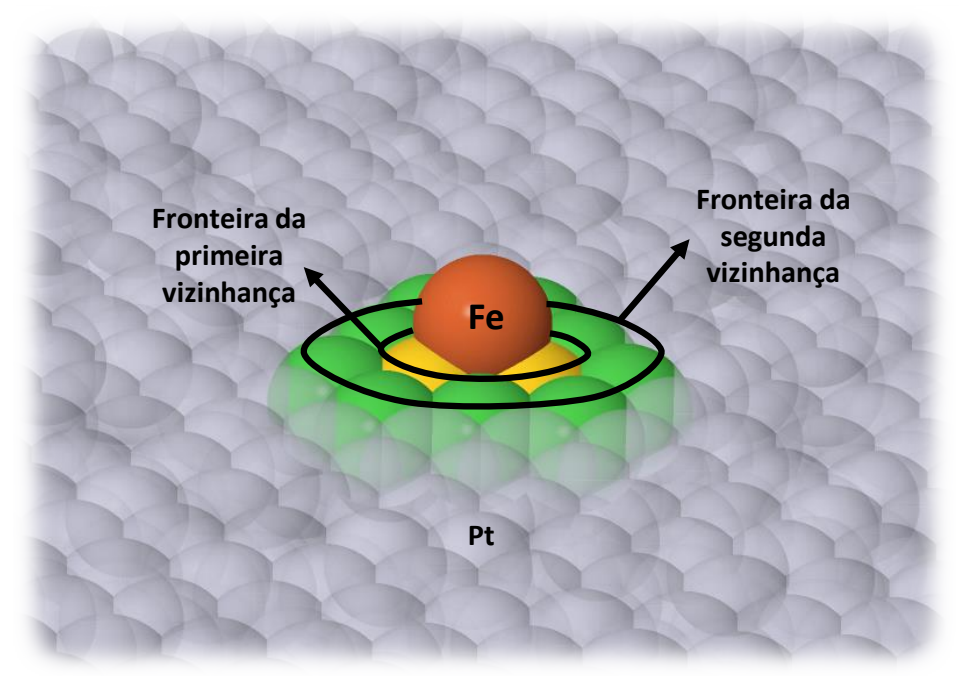

Figura 3.5: Representação esquemática de um adátomo de Fe depositado na superfície de $\operatorname{Pt}(111)$, em localização aproximadamente central do cluster. Os primeiros e segundos vizinhos da impureza em $\operatorname{Pt}(S)$ são evidenciados pelas cores amarela e verde das esferas, respectivamente. Os átomos de $\mathrm{Pt}$ são ilustrados pelas esferas cinzas translúcidas, enquanto o átomo de Fe é indicado pela esfera laranja, ambos sinalizados pelas legendas na figura.

\subsubsection{Cálculo da interação de troca $\left(\mathcal{J}_{i j}\right)$}

Oguchi et al. [149] apresentaram em 1983 um modo de calcular o parâmetro de troca baseado na diferença em energia de um determinado par de átomos quando em configurações FM e AFM, onde o parâmetro de Heisenberg é expresso como

$$
\mathcal{J}_{i j}=-\frac{\left(\Delta E_{F M}-\Delta E_{A F M}\right)}{2 S^{2}}
$$

sendo $S$ a magnitude do spin atômico ${ }^{1}$. A Eq. 3.28 é uma aplicação direta da Eq. 2.2, uma vez que os ângulos $\theta$ entre os spins valem $0 \mathrm{rad}$ e $\pi \mathrm{rad}$ para as configurações FM e AFM, respectivamente, de modo que $\Delta E_{F M}=-\mathcal{J}_{i j} S^{2}, \Delta E_{A F M}=\mathcal{J}_{i j} S^{2}$, e $\mathcal{H}_{\text {exc }}=$ $\left(\Delta E_{F M}-\Delta E_{A F M}\right)=-2 \mathcal{J}_{i j} S^{2}$.

Em 1987, Liechtenstein et al. [77] propuseram uma abordagem na qual a simples diferença entre as energias nas configurações FM e AFM de um par de átomos não é mais suficiente para descrever quantitativamente $\mathcal{J}_{i j}$ - como na Eq. 3.28. Ao invés disso, os parâmetros de troca são obtidos por pequenas rotações dos momentos magnéticos nos sítios atômicos a partir da configuração que caracteriza o estado fundamental do sistema.

\footnotetext{
${ }^{1}$ Se o material é formado por apenas um tipo de átomo, para sítios $i$ e $j$ tem-se, obviamente, a seguinte relação $\left|\mathbf{S}_{i}\right|=\left|\mathbf{S}_{j}\right|=S$, e pode-se assumir a existência de uma única magnitude de spin.
} 
É importante enfatizar, no entanto, que o método de Liechtenstein-Katsnelson-AntropovGubanov (LKAG) é deduzido a partir da forma clássica da Hamiltoniana definida pela Eq. 2.2. Assim, define-se $\mathcal{H}_{\text {exc }}$ segundo os versores $\mathbf{e}_{i}$ que apontam na direção dos momentos magnéticos de spin, produzindo $\mathcal{H}_{e x c}=-2 \sum_{i<j} \mathcal{J}_{i j} \mathbf{e}_{i} \cdot \mathbf{e}_{j}$. Uma variação angular $\theta$ do momento de spin em um determinado sítio $m$ (estado excitado) provoca, a priori, não apenas uma mudança local na energia, mas modifica as interações daquele spin com o restante da estrutura. Assim, a esta rotação deve-se somar todas as contribuições dos demais sítios da rede. Sabendo que $\mathbf{e}_{i} \cdot \mathbf{e}_{m} \equiv \cos \theta$, então:

$$
\delta E_{m}=2 \sum_{i} \mathcal{J}_{m i}(1-\cos \theta)
$$

onde $\mathcal{J}_{m i}$ representa o parâmetro de Heisenberg entre os sítios genéricos $m$ e $i$. Se o ângulo $\theta$ for suficientemente pequeno, então $(1-\cos \theta) \sim \frac{1}{2} \theta^{2}$ e a Eq. 3.29 pode ser reescrita como $\delta E_{m} \sim \sum_{i} \mathcal{J}_{m i} \theta^{2}$. Ou ainda, considerando $J_{m}=\sum_{i} \mathcal{J}_{m i}$ como uma constante efetiva de troca local no sítio $n, \delta E_{m} \sim J_{m} \theta^{2}$. É também necessário determinar a interação local $\mathcal{J}_{n i}$ entre o par de átomos nos sítios $n$ e $i$, obtida variando-se os momentos de spin destes átomos em direções opostas $\left( \pm \frac{\theta}{2}\right)$, e subtraindo, em seguida, suas energias de interação com o ambiente:

$$
\delta E_{\mathrm{par}}=\delta E_{n i}-\delta E_{n}-\delta E_{i} \sim \frac{1}{2} \mathcal{J}_{n i} \theta^{2}
$$

Logo, a conexão entre o cálculo dos parâmetros de troca $\mathcal{J}_{i j}$ e os métodos de primeiros princípios é feita a partir da determinação de variações na energia total do sistema devidas a pequenas perturbações na densidade de spin. Na referência [77], Liechtenstein et al. empregam o Teorema da Força Local (TFA) de Andersen [150, 151], que relaciona a alteração da energia total $\delta E$ com a soma das variações nas energias de partícula única $(\epsilon)$ para os estados ocupados no potencial fixo do estado fundamental. Em primeira ordem de perturbação nas densidades de carga e de spin, chega-se na seguinte expressão [77]

$$
\delta E=-\int_{-\infty}^{\varepsilon_{F}} \varepsilon[\delta n(\varepsilon)] d \varepsilon=-\int_{-\infty}^{\varepsilon_{F}}[\delta N(\varepsilon)] d \varepsilon
$$

onde $n(\varepsilon)=\frac{d N(\varepsilon)}{d \varepsilon}$ é a densidade de estados eletrônicos, $N(\varepsilon)$ é o número de estados, e $\varepsilon_{F}$ é a energia de Fermi. Portanto, a fim de calcular $\delta N(\varepsilon)$ na Eq. 3.31 correspondente a rotações dos momentos de spin nos sítios $1,2, \ldots, k$ do sistema, emprega-se a abordagem denominada espalhamento múltiplo (ou multiple scattering) [152]1․ Assim, de acordo

\footnotetext{
${ }^{1} \mathrm{~A}$ apresentação da equação de Liechtenstein et al. [77] tem caráter introdutório nesta monografia. Portanto, não será apresentada a abordagem multiple scattering, pois foge do escopo do texto. Uma descrição detalhada deste método pode ser encontrada nas referências [152, 153].
} 
com a fórmula de Lloyd [152]

$$
N(\varepsilon)=N_{0}(\varepsilon)+\frac{1}{\pi} \mathcal{I} m \operatorname{Tr} \ln \tau
$$

na qual $N_{0}(\varepsilon)$ é o número de estados no sistema sem perturbações na densidade de spin, e $\tau$ é o chamado scattering path operator [153], que compreende todos os eventos de espalhamento entre dois sítios específicos. A substituição da Eq. 3.32 na Eq. 3.31 e subsequentes desenvolvimentos teóricos considerando-se a perturbação do sistema pela rotação local dos spins resultam na fórmula de LKAG, que depende de sua função de Green.

No método RS-LMTO-ASA, o cálculo da interação de troca magnética segue uma abordagem bem similar à adotada por Liechtenstein et al., por meio da relação [154] ( $\varepsilon$ é a variável de energia):

$$
\mathcal{J}_{i j}=\frac{1}{4 \pi} \mathcal{I} m \operatorname{Tr} \int_{-\infty}^{\varepsilon_{F}} d \varepsilon\left[\delta_{i}(\varepsilon) G_{i j}^{\Uparrow}(\varepsilon) \delta_{j}(\varepsilon) G_{j i}^{\Downarrow}(\varepsilon)\right]
$$

Note que os parâmetros $\mathcal{J}_{i j}$ são descritos em termos das funções de Green $G_{i j}^{\sigma \sigma}$, cuja dimensão é expressa pelo inverso da energia, e caracteriza-se como o propagador para os elétrons com spin $\sigma$ entre os sítios $i$ e $j$. Por sua vez, $\delta_{i}(\varepsilon)$ é uma matriz diagonal dentro do conjunto dos orbitais ( $s, p$ e $d$ ), composta pelos elementos:

$$
\delta_{l i}=\frac{C_{l i}^{\downarrow} \Delta_{l i}^{\uparrow}-C_{l i}^{\uparrow} \Delta_{l i}^{\downarrow}+\left(\Delta_{l i}^{\downarrow}-\Delta_{l i}^{\uparrow}\right) E}{\left(\Delta_{l i}^{\downarrow} \Delta_{l i}^{\uparrow}\right)^{1 / 2}},
$$

onde $l$ são os números quânticos orbitais $(l=0,1$ e 2$)$ associados aos elétrons, e $\left\{C_{l i}^{\sigma}, \Delta_{l i}^{\sigma}\right\}$ são os elementos dos parâmetros de potencial do sítio $i$ na representação ortogonal (veja a seção B.1.7). A quantidade $\delta_{i}(E)$ é associada com o exchange splitting local no sítio $i$. Se $\Delta_{i}^{\downarrow}=\Delta_{i}^{\uparrow}$, a Eq. 3.34 torna-se independente da energia, e é reduzida a uma diferença entre os centros das bandas de energia dos spins $\uparrow$ e $\downarrow$ - condição frequentemente usada nas parametrizações TB.

Nos cálculos realizados no capítulo 5 , os resultados de $\mathcal{J}_{i j}$ (Eq. 3.33) foram utilizados na verificação qualitativa da estabilidade das configurações magnéticas investigadas. Com este objetivo, partiu-se sempre de um determinado ordenamento magnético entre dois átomos: FM ou AFM (veja a seção 2.2). Assim, caso o valor da interação de troca seja positivo $\left(\mathcal{J}_{i j}>0\right)$ para um estado inicialmente FM, esta configuração é estável; por outro lado, um valor negativo $\left(\mathcal{J}_{i j}<0\right)$ indica a instabilidade do alinhamento paralelo entre os 
momentos de spin nesta situação. Este mesmo procedimento pode ser usado para uma configuração inicial AFM. Ademais, nos casos em que os valores de $\mathcal{J}_{i j}$ são negativos tanto nas configurações FM quanto AFM, os resultados sugerem a possibilidade do ordenamento não-colinear como estado fundamental [155].

\subsubsection{Cálculo da interação de Dzyaloshinskii-Moriya}

No contexto do método RS-LMTO-ASA, o desenvolvimento de uma expressão formal para o cálculo das interações de DM seguiu um esquema semelhante ao realizado por Liechtenstein et al. [77] para os acoplamentos de troca $\left(\mathcal{J}_{i j}\right)$. A implementação deste tipo de cálculo no pacote computacional utilizado no presente trabalho é bastante recente, sendo esta uma das primeiras aplicações a sistemas metálicos "reais" organizados em multicamadas. Analogamente à referência [77], as propriedades eletrônicas do sistema são obtidas através da Teoria de Espalhamento Múltiplo (TEM), uma técnica bastante eficiente para tal finalidade. Isto porque o formalismo da TEM relaciona-se diretamente à função de Green do sistema em questão, podendo então ser aplicada a diversas classes de problemas, dentre elas as investigações em magnetismo, transporte, e espectroscopia. Assim, parte do desenvolvimento apresentado nesta seção está baseado nas referências $[156,157]$.

Em poucas palavras, podemos definir a Teoria do Espalhamento Múltiplo como aquela que trata de descrever a propagação de uma onda (no caso, um elétron) através de um conjunto de centros de espalhamento (no caso, os íons ou sítios atômicos do material). A equação fundamental para a TEM escalar relativística pode ser escrita da seguinte maneira [153]:

$$
\left(\tau_{i j}^{-1}\right)_{L \sigma, L^{\prime} \sigma^{\prime}}=p_{i L \sigma \sigma^{\prime}} \delta_{i j} \delta_{L L^{\prime}}-G_{i j, L L^{\prime}}^{0} \delta_{\sigma \sigma^{\prime}}
$$

onde $\tau_{i j}$ denota o chamado scattering path operator, e $\mathbf{p}_{i}=\mathbf{t}_{i}^{-1}$ é o inverso do chamado single site scattering operator. Fisicamente, a matriz $\mathbf{t}$ descreve o espalhamento eletrônico em um potencial de único sítio (p. e., de um único íon na rede), enquanto o operador $\tau_{i j}$ é aquele que descreve todos os eventos de espalhamento possíveis entre dois sítios $i$ e $j$ do sistema (distintos ou não). Na Eq. 3.35, empregamos as notações $L=(l, m)$ para o par de números quânticos de momento angular e magnético, $\sigma$ para o índice de spin, e $\mathbf{G}_{i j}^{0}$ para a função de Green relativa ao sistema não-interagente (isto é, com Hamiltoniana $\mathcal{H}^{0}$, e energia potencial nula). Como $\mathbf{G}_{i j}^{0}$ é calculada a partir da Hamiltoniana de partícula livre, então será independente de spin. Nas expressões a seguir, os termos em negrito 
representarão as quantidades dependentes tanto de spin como no orbital (gerando matrizes $18 \times 18$ na base reduzida de funções $s p d$ ), enquanto os termos sem o destaque em negrito são aqueles dependentes apenas do orbital, constituindo matrizes $9 \times 9$ na base de funções spd.

Considerando que os spins do sistema em questão podem assumir quaisquer direções dentro da esfera unitária (hipótese da não-colinearidade), então podemos separar a matriz t em duas principais contribuições: uma diagonal, independente de spin, e outra nãodiagonal, dependente de spin. Explicitamente:

$$
\mathbf{t}_{i}(\varepsilon)=t_{i}^{0}(\varepsilon) \mathbb{1}+t_{i}(\varepsilon) \mathbf{e}_{i} \cdot \boldsymbol{\sigma}
$$

onde $\varepsilon$ é a variável de energia, $\mathbf{e}_{i}$ é um vetor unitário na direção local do momento de spin no sítio $i, \boldsymbol{\sigma}$ representa o vetor formado pelas matrizes de Pauli, isto é, $\boldsymbol{\sigma} \equiv\left(\sigma_{x}, \sigma_{y}, \sigma_{z}\right)$, e $\mathbb{1}$ é a matriz unitária no espaço de spin. É importante notar que, com essa separação, $t_{i}^{0}$ denota a parte não-magnética da matriz $\mathbf{t}$, e, portanto, está associada à contribuição da carga. Estando os operadores $\mathbf{p}$ e $\mathbf{t}$ relacionados, podemos introduzir uma separação de contribuições análoga à Eq. 3.36 para $\mathbf{p}$, a saber:

$$
\mathbf{p}_{i}(\varepsilon)=p_{i}^{0}(\varepsilon) \mathbb{1}+p_{i}(\varepsilon) \mathbf{e}_{i} \cdot \boldsymbol{\sigma}
$$

Assim, como resultado de pequenas rotações $\delta \mathbf{e}_{i}$ no momento de spin do $i$-ésimo átomo (sem, no entanto, mudar a sua magnitude), teremos também uma variação local no operador $\mathbf{p}_{i}$, que pode ser expressa por

$$
\delta \mathbf{p}_{i}=p_{i} \delta \mathbf{e}_{i} \cdot \boldsymbol{\sigma}
$$

Finalmente, o scattering path operator terá também uma forma similar às matrizes $\mathbf{p}$ e t, podendo ser escrita como a soma de duas contribuições

$$
\tau_{i j}=T_{i j}^{0} \mathbb{1}+\mathbf{T}_{i j} \cdot \boldsymbol{\sigma}
$$

sendo $T_{i j}^{0}$ a componente de carga, e $\mathbf{T}_{i j}=\left(T_{i j}^{x}, T_{i j}^{y}, T_{i j}^{z}\right)$ a componente de spin do operador $\tau_{i j}$. Nota-se ainda que, no limite de uma configuração colinear, naturalmente $\mathbf{T}_{i j} \rightarrow\left(0,0, T_{i j}^{z}\right)$, e as componentes do scattering path operator para os canais majo- 
ritário e minoritário de spin serão consequentemente definidas como $T_{i j}^{\uparrow}=\left(T_{i j}^{0}+T_{i j}^{z}\right)$ e $T_{i j}^{\downarrow}=\left(T_{i j}^{0}-T_{i j}^{z}\right)$, respectivamente. Portanto, as quantidades necessárias para o cálculo da variação na energia total $\delta E_{i j}$ em um par fixo de momentos de spin nos sítios $i$ e $j$, quando esses momentos são ligeiramente desviados da sua configuração magnética do estado fundamental, já foram todas determinadas. É possível mostrar que essa variação na energia depende da densidade de estados $N(\varepsilon)$, e, de acordo com a fórmula de Lloyd [152], o valor de $N(\varepsilon)$ é matematicamente expresso em termos do scattering path operator. Realizando os mesmos procedimentos formais reportados na referência [77], e considerando uma rotação simultânea dos spins em ambos os sítios, finalmente escreve-se $\delta E_{i j}$ da seguinte maneira [158]:

$$
\delta E_{i j}=-\frac{1}{\pi} \int_{-\infty}^{\varepsilon_{F}} d \varepsilon \operatorname{Im} \operatorname{Tr}\left(\delta \mathbf{p}_{i} \tau_{i j} \delta \mathbf{p}_{j} \tau_{j i}\right)
$$

na qual $\varepsilon_{F}$ simboliza a energia de Fermi do sistema. A interpretação física dessa equação não é trivial, mas podemos compreender a variação da energia total $\delta E_{i j}$ como uma quantidade dependente dos espalhamentos eletrônicos tanto no próprio sítio quanto entre sítios distintos da rede no material. Uma representação do espalhamento eletrônico entre dois sítios $i$ e $j$ associado à rotação simultânea dos momentos de spin em ângulos $\theta_{i}$ e $\theta_{j}$ é apresentada na Fig. 3.6, a seguir.

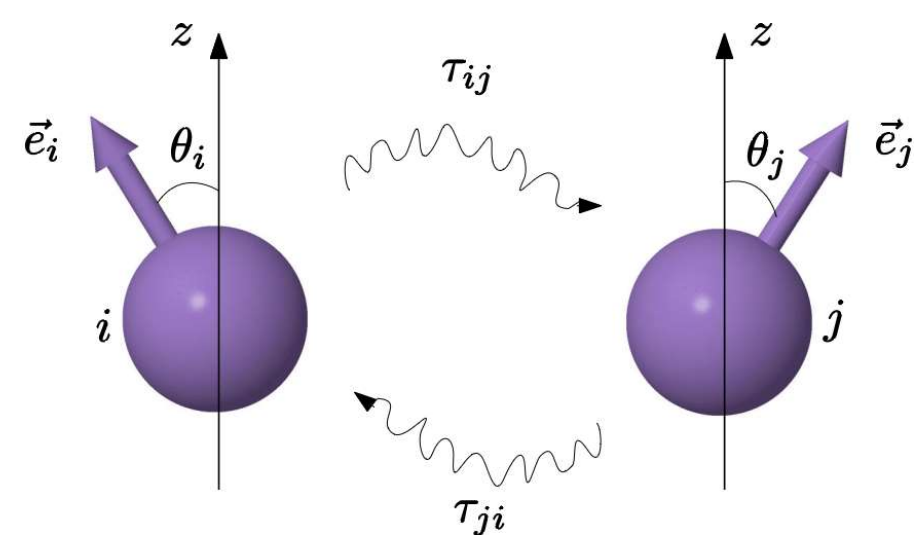

Figura 3.6: Interação eletrônica entre dois sítios $i$ e $j$ no sistema, com momentos locais de direções $\mathbf{e}_{i}$ e $\mathbf{e}_{j}$. A rotação em relação ao estado colinear (em relação ao eixo $z$ ) está associada aos espalhamentos eletrônicos entre os sítios, cujos eventos possíveis são quantificados pelos operadores $\tau_{i j}$ e $\tau_{j i}$. Figura gentilmente fornecida por Ramon Cardias.

Considerando, entretanto, os efeitos do acoplamento spin-órbita no formalismo apresentado acima (veja o Apêndice $\mathbf{C}$ para mais detalhes) finalmente encontramos (após 
alguns cancelamentos) que

$$
\delta E_{i j}^{\prime}=-2\left(A_{i j}^{00}-\sum_{\mu=x, y, z} A_{i j}^{\mu \mu}\right) \delta \mathbf{e}_{i} \cdot \delta \mathbf{e}_{j}-4 \sum_{\mu, \nu=x, y, z} \delta e_{i}^{\mu} A_{i j}^{\mu \nu} \delta e_{j}^{\nu}-2 \mathbf{D}_{i j} \cdot\left(\delta \mathbf{e}_{i} \times \delta \mathbf{e}_{j}\right)
$$

com o operador $A_{i j}$ e o vetor $\mathbf{D}_{i j}$ definidos pelos elementos $(\mu=x, y, z)$

$$
\begin{array}{r}
A_{i j}^{\alpha \beta}=\frac{1}{\pi} \int_{-\infty}^{\varepsilon_{F}} d \varepsilon \operatorname{Im} \operatorname{Tr}\left(p_{i} T_{i j}^{\alpha} p_{j} T_{j i}^{\beta}\right) \\
D_{i j}^{\mu}=\frac{1}{\pi} \int_{-\infty}^{\varepsilon_{F}} d \varepsilon \operatorname{Re} \operatorname{Tr}\left(p_{i} T_{i j}^{0} p_{j} T_{j i}^{\mu}-p_{j} T_{j i}^{0} p_{i} T_{i j}^{\mu}\right) .
\end{array}
$$

Observa-se que, por estruturar-se no produto escalar com o termo vetorial $\left(\delta \mathbf{e}_{i} \times \delta \mathbf{e}_{j}\right)$, o vetor $\mathbf{D}_{i j}$ (Eqs. 3.41 e 3.42) deve justamente representar o parâmetro de interação de DM. Além disso, a quantidade $\left(A_{i j}^{00}-\sum_{\mu=x, y, z} A_{i j}^{\mu \mu}\right)$, que se constitui como uma constante de proporcionalidade ao produto $\left(\delta \mathbf{e}_{i} \cdot \delta \mathbf{e}_{j}\right)$, deve ser interpretada fisicamente como o próprio parâmetro de interação de troca $\left(\mathcal{J}_{i j}\right)$, obtido para um sistema no qual foi assumida a hipótese da não-colinearidade entre os spins. Isso significa, de uma maneira simplificada, que o cálculo dos parâmetros de acoplamento magnético neste modelo dependem apenas da obtenção das matrizes $p$ e $T$ associadas ao sítios $i$ e $j$ de interesse no sistema.

Apesar de se constituir a linguagem natural para o método no espaço-k de KorringaKohn-Rostoker (KKR) [159, 160], a TEM não possui uma conexão direta (trivial) com o método LMTO, e, portanto, é necessário traduzir os elementos apresentados na Eq. 3.42 para o formalismo utilizado no presente trabalho. Uma das maneiras de fazê-lo foi reportada por Schilfgaarde [161] e Antropov [162], em 1999. Os autores consideraram que as matrizes $p$ e $T$, aqui com os índices orbitais suprimidos, podem ser fatoradas em termos dos parâmetros de potencial no formalismo LMTO da seguinte maneira (válido para a representação ortogonal):

$$
T=\Delta^{\frac{1}{2}} G \Delta^{\frac{1}{2}} ; p=\frac{C-\varepsilon}{\Delta}
$$

sendo $G$ a função de Green e $\varepsilon$ a variável de energia. Fazendo essa substituição para cada elemento do operador $A_{i j}$ (Eq. 3.42), e, lembrando que no limite colinear podemos definir 
matrizes $T_{i j}^{\uparrow}$ e $T_{i j}^{\downarrow}$ para os canais majoritário e minoritário de spin, além do fato de que, nesse regime magnético, o termo $\left(A_{i j}^{00}-\sum_{\mu=x, y, z} A_{i j}^{\mu \mu}\right)$ tende a $\left(A_{i j}^{00}-A_{i j}^{z z}\right)$, é possível retomar a expressão anteriormente apresentada para o cálculo de $\mathcal{J}_{i j}$ (seção 3.2.5) - e que foi reportada pela primeira vez no contexto do método RS-LMTO-ASA na referência $[154]$.

As primeiras publicações envolvendo o cálculo do vetor de DM para sistemas magnéticos via RS-LMTO-ASA ocorreram em 2020 [157, 163], para tratar de átomos de Cr e Mn depositados em $\mathrm{Au}(111)$ e $\mathrm{Ag}(111)$ e do magnetismo do bulk de $\mathrm{Mn}_{3} \mathrm{Sn}$. 


\section{Capítulo 4}

\section{Dinâmica atomística de spins}

Com a obtenção dos parâmetros de interação magnética via cálculos de primeiros princípios, em particular a interação isotrópica de Heisenberg e os acoplamentos anisotrópicos de DM, torna-se possível fazer uma conexão com a dinâmica de spins na aproximação atomística - tópico que será discutido neste capítulo. Essa conexão permite, com boa aproximação, investigar a resposta temporal da magnetização do sistema em análise, partindo da estrutura eletrônica calculada para seu estado fundamental. A equação principal que conduz a dinâmica de spins será derivada e discutida em detalhes, bem como o tamanho e a escala temporal adequadas para essas simulações.

Os cálculos apresentados neste capítulo foram realizados com a implementação computacional mais recente do pacote $\boldsymbol{U p p}$ sala Atomistic Spin Dynamics (UppASD) [164-166], desenvolvido na Divisão de Teoria dos Materiais da Universidade de Uppsala (Suécia).

\subsection{Equação de movimento}

A evolução temporal do $i$-ésimo momento magnético atômico $\left(\mathbf{m}_{i}\right)$ em um sistema é governada pela equação de movimento de Landau-Lifshitz-Gilbert (LLG) [63], a saber:

$$
\frac{d \mathbf{m}_{i}}{d t}=-\gamma_{L} \mathbf{m}_{i} \times\left(\mathbf{B}_{i}+\mathbf{B}_{i}^{s}\right)-\gamma_{L} \frac{\alpha}{m_{i}} \mathbf{m}_{i} \times\left[\mathbf{m}_{i} \times\left(\mathbf{B}_{i}+\mathbf{B}_{i}^{s}\right)\right]
$$

onde $\mathbf{B}_{i}=\mathbf{B}_{i}(t)$ é o campo magnético efetivo sentido pelo $i$-ésimo átomo da rede, e $\mathbf{B}_{i}^{s}$ representa um campo magnético estocástico. Além desses termos, a Eq. 4.1 também leva em conta a constante (isotrópica) de damping de Gilbert $(\alpha)$, e a razão giromagnética 
eletrônica $\gamma$, cujo valor está indiretamente incluído no parâmetro $\gamma_{L}$, definido como

$$
\gamma_{L}=\frac{\gamma}{\left(1+\alpha^{2}\right)}
$$

É importante notar que o parâmetro de damping de Gilbert, por fundamentalmente reger a magnitude do segundo termo da Eq. 4.1 (isto é, se $\alpha=0$, então $\frac{d \mathbf{m}_{i}}{d t}=-\gamma \mathbf{m}_{i} \times$ $\left(\mathbf{B}_{i}+\mathbf{B}_{i}^{s}\right)$ ), fisicamente permite que a energia magnética e o momento angular sejam dissipados da caixa de simulação. Dessa forma, seu valor controla a rapidez com que o sistema de spins pode atingir um eventual equilíbrio termodinâmico, e afeta diretamente a velocidade com a qual o material de interesse pode operar [167]. Um exemplo claro da relevância do valor de $\alpha$ para as simulações é encontrado nos sistemas que apresentam rápidas mudanças na magnetização (da ordem de 10 ps ou menos) [168], promissores para o uso em tecnologias de leitura/escrita de informação baseadas em materiais magnéticos. A Figura 4.1, extraída da Ref. [165], mostra a evolução do momento magnético m no tempo sob a ação de um campo magnético efetivo $\mathbf{B}$.

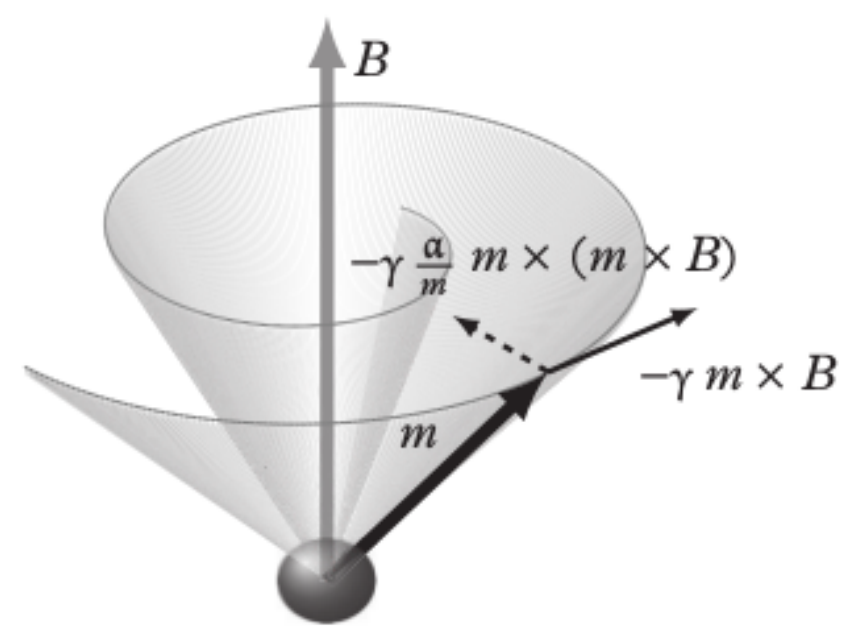

Figura 4.1: Representação do movimento amortecido de precessão realizado pelo momento magnético $\mathbf{m}$ no tempo, tal como expresso pela Eq. 4.1 [165]. A seta mais grossa indica a direção instantânea de $\mathbf{m}$, enquanto as setas mais finas indicam as componentes perpendicular e paralela ao movimento.

Um aspecto fundamental da dinâmica atomística de spins é a definição do campo magnético efetivo sentido pelo $i$-ésimo spin. Naturalmente, esse campo deve conter as interações experimentadas pelos momentos magnéticos dos átomos - e, claro, o efeito de um campo magnético externo aplicado ao sistema, se houver. Dentre as interações magnéticas, 
inevitavelmente a mais importante será a interação de troca $\mathcal{J}$. Entretanto, para alguns sistemas (sobretudo aqueles que apresentam quebra na simetria de inversão e elevado acoplamento spin-órbita [169]), o termo de Dzyaloshinskii-Moriya é não-desprezível, e precisa ser levado em conta no cálculo do campo magnético efetivo. Analogamente, demais termos como a anisotropia magnética, a interação dipolar (de longo alcance), o termo de interação four-spin [46], entre outros, também podem ser considerados na determinação do campo efetivo. Assim, a maneira mais geral e direta possível para determinar $\mathbf{B}_{i}$ em cada caso é expressá-lo em termos de uma Hamiltoniana efetiva de spin $(\mathcal{H})$. Matematicamente:

$$
\mathbf{B}_{i}=-\frac{\partial \mathcal{H}}{\partial \mathbf{m}_{i}}
$$

Para efeitos práticos, a Hamiltoniana de spin pode ser escrita como (desconsiderandose o termo de anisotropia)

$$
\mathcal{H}=\underbrace{-\sum_{i \neq j} \mathcal{J}_{i j}\left(\mathbf{m}_{i} \cdot \mathbf{m}_{j}\right)}_{\begin{array}{c}
\text { Termo de } \\
\text { interação de troca } \\
\text { (exchange) }
\end{array}}-\underbrace{\sum_{i \neq j} \mathbf{D}_{i j} \cdot\left(\mathbf{m}_{i} \times \mathbf{m}_{j}\right)}_{\begin{array}{c}
\text { Termo de } \\
\text { Dzyaloshinskii-Moriya } \\
\text { (anisotrópico) }
\end{array}}-\underbrace{-\sum_{i} \mathbf{B}_{\text {ext }} \cdot \mathbf{m}_{i}}_{\begin{array}{c}
\text { Termo de } \\
\text { interação Zeeman }
\end{array}},
$$

que está, pela Eq. 4.4, sujeito a um campo magnético externo $\mathbf{B}_{\text {ext. }}$. Resolvendo, portanto, a Eq. 4.3 para o $i$-ésimo átomo, teremos $3 N$ equações de movimento análogas à Eq. 4.1 para $N$ sítios não-equivalentes pertencentes ao sistema de interesse. Obviamente, mais termos de interação ainda podem ser adicionados à Hamiltoniana da Eq. 4.4 (tais como a anisotropia magnética), desde que sejam fornecidos os parâmetros de acoplamento correspondentes.

A forma explícita das equações de LLG para a Hamiltoniana de spin definida na Eq. 4.4 pode ser facilmente obtida. Pela expressão do campo efetivo local (Eq. 4.3), e considerando que $\mathbf{m}_{i, j}=\left(m_{i, j}^{x}, m_{i, j}^{y}, m_{i, j}^{z}\right), \mathbf{D}_{i j}=\left(D_{i j}^{x}, D_{i j}^{y}, D_{i j}^{z}\right)$, e $\mathbf{B}_{\text {ext }}=\left(B_{\text {ext }}^{x}, B_{\text {ext }}^{y}, B_{\text {ext }}^{z}\right)$, 
então as componentes do campo $\mathbf{B}_{i}$ devem obedecer as seguintes relações

$$
\begin{aligned}
& B_{i}^{x}=-\frac{\partial \mathcal{H}}{\partial m_{i}^{x}}=\sum_{i \neq j} \mathcal{J}_{i j} m_{j}^{x}+\sum_{i \neq j}\left(D_{i j}^{z} m_{j}^{y}-D_{i j}^{y} m_{j}^{z}\right)+B_{\mathrm{ext}}^{x} \\
& B_{i}^{y}=-\frac{\partial \mathcal{H}}{\partial m_{i}^{y}}=\sum_{i \neq j} \mathcal{J}_{i j} m_{j}^{y}+\sum_{i \neq j}\left(D_{i j}^{x} m_{j}^{z}-D_{i j}^{z} m_{j}^{x}\right)+B_{\mathrm{ext}}^{y} \\
& B_{i}^{z}=-\frac{\partial \mathcal{H}}{\partial m_{i}^{z}}=\sum_{i \neq j} \mathcal{J}_{i j} m_{j}^{z}+\sum_{i \neq j}\left(D_{i j}^{y} m_{j}^{x}-D_{i j}^{x} m_{j}^{y}\right)+B_{\mathrm{ext}}^{z}
\end{aligned}
$$

Sabendo que, na Eq. 4.1, sempre podemos considerar um campo magnético total dependente do tempo $\left(\mathbf{B}_{\text {tot }}\right)$ formado pelas contribuições efetiva $\left(\mathbf{B}_{i}\right)$ e estocástica $\left(\mathbf{B}_{i}^{s}\right)$, isto é, $\mathbf{B}_{\text {tot }}(t)=\mathbf{B}_{i}(t)+\mathbf{B}_{i}^{s}(t)$, então o momento magnético do $i$-ésimo átomo estará sujeito às seguintes equações de movimento:

$$
\begin{aligned}
\frac{d m_{i}^{x}}{d t} & =-\gamma_{L}\left(m_{i}^{z} B_{\mathrm{tot}}^{y}-m_{i}^{y} B_{\mathrm{tot}}^{z}\right)-\gamma_{L} \frac{\alpha}{m_{i}}\left[\left(\left(m_{i}^{x}\right)^{2}-m_{i}^{2}\right) B_{\mathrm{tot}}^{x}+m_{i}^{y} B_{\mathrm{tot}}^{y}+m_{i}^{z} B_{\mathrm{tot}}^{z}\right] \\
\frac{d m_{i}^{y}}{d t} & =-\gamma_{L}\left(m_{i}^{x} B_{\mathrm{tot}}^{z}-m_{i}^{z} B_{\mathrm{tot}}^{x}\right)-\gamma_{L} \frac{\alpha}{m_{i}}\left[m_{i}^{x} B_{\mathrm{tot}}^{x}+\left(\left(m_{i}^{y}\right)^{2}-m_{i}^{2}\right) B_{\mathrm{tot}}^{y}+m_{i}^{z} B_{\mathrm{tot}}^{z}\right] \\
\frac{d m_{i}^{z}}{d t} & =-\gamma_{L}\left(m_{i}^{y} B_{\mathrm{tot}}^{x}-m_{i}^{x} B_{\mathrm{tot}}^{y}\right)-\gamma_{L} \frac{\alpha}{m_{i}}\left[m_{i}^{x} B_{\mathrm{tot}}^{x}+m_{i}^{y} B_{\mathrm{tot}}^{y}+\left(\left(m_{i}^{z}\right)^{2}-m_{i}^{2}\right) B_{\mathrm{tot}}^{z}\right],
\end{aligned}
$$

nas quais se nota uma clara interdependência. Essas expressões podem ser resolvidas com o auxílio de métodos numéricos, constituindo o tempo uma variável discreta na evolução dinâmica do sistema. No caso específico do pacote computacional UppASD, o método utilizado para resolver as equações estocásticas definidas em $\mathbf{4 . 6}$ foi proposto por J. Mentink et al. [170], com o intervalo temporal típico de $\Delta t=10^{-16} \mathrm{~s}$.

\subsection{Efeitos de temperatura: dinâmica de Langevin}

$\mathrm{Na}$ seção anterior, explicamos brevemente o movimento de precessão do spin e a sua dissipação da caixa de simulação, controlado pelo fator de damping de Gilbert $(\alpha)$. Também mencionamos que ao campo magnético efetivo $\mathbf{B}_{i}$, ao qual o $i$-ésimo átomo da rede está sujeito, adicionamos um termo estocástico $\mathbf{B}_{i}^{s}$, embora não tenhamos mencionado qualquer propriedade deste campo. Assim, é importante esclarecer que o campo estocástico $\mathbf{B}_{i}^{s}$ é justamente o termo responsável por introduzir os efeitos de tempera- 
tura finita $(T>0)$ no sistema. A introdução deste campo flutuante na equação de LLG (Eq. 4.1) vem da necessidade de se levar em conta os efeitos da interação do momento local $\mathbf{m}_{i}$ com diversos outros graus de liberdade no sistema, tais como fônons, elétrons de condução, spins nucleares, entre outros, que causam flutuações na orientação do momento magnético ao longo do tempo [171]. De certa forma, esses graus de liberdade também estão relacionados ao amortecimento da precessão do spin, haja vista que os processos de dissipação (associado à constante de damping) e flutuação dos momentos magnéticos são ambos manifestações de um mesmo conjunto de interações do momento $\mathbf{m}_{i}$ com o seu ambiente. Portanto, por considerar o efeito de graus de liberdade ocultos por meio da introdução de um termo estocástico, dizemos que, neste trabalho, utilizamos a dinâmica de Langevin para encontrar as soluções dinâmicas da magnetização nos sistemas de interesse.

Os campos estocásticos flutuantes $\mathbf{B}_{i}^{s}$ são funções gaussianas modeladas como um "ruído branco". Supõe-se um formato gaussiano para essas funções, pois as flutuações na magnetização devem emergir de interações com um grande número de graus de liberdade, que apresentam essencialmente as mesmas propriedades estatísticas. Por outro lado, com a escolha do modelo de "ruído branco", essas funções estocásticas não são correlacionadas nem no tempo, nem no espaço, como veremos adiante. Isso pode ser matematicamente expresso pelos seguintes critérios [171]:

$$
\begin{array}{r}
\left\langle\mathbf{B}_{i}^{s}(t)\right\rangle=0 \\
\left\langle B_{i \mu}^{s}(t) B_{j \nu}^{s}\left(t^{\prime}\right)\right\rangle=2 D \delta_{\mu \nu} \delta\left(t-t^{\prime}\right) \\
D=\frac{\alpha}{\left(1+\alpha^{2}\right)} \frac{k_{B} T}{\mu_{B} m_{i}},
\end{array}
$$

onde $\langle\ldots\rangle$ denota a média sobre todas as diferentes realizações do campo flutuante. Assim, o primeiro critério apresentado na Eq. 4.7 mostra que a média temporal de $\mathbf{B}_{i}^{s}$ deve ser sempre nula. Já o delta de Dirac $\delta\left(t-t^{\prime}\right)$, presente na segunda condição, bem como o delta de Kronecker $\delta_{\mu \nu}(\operatorname{com}\{\mu, \nu\}$ representando as coordenadas espaciais $x, y, z)$, caracterizam o campo estocástico como não-correlacionado tanto no tempo quanto no espaço. A relação com a temperatura é formalmente expressa pela dependência da função de correlação $\left\langle B_{i \mu}^{s}(t) B_{j \nu}^{s}\left(t^{\prime}\right)\right\rangle$ com o parâmetro $D$, que, por sua vez, é diretamente proporcional a $T$. É também importante notar que $D$ é dependente do parâmetro de damping de Gilbert, $\alpha$, sendo que o vínculo entre $D$ e a dissipação de energia no movimento dos spins é estabelecido pelo Teorema de Flutuação-Dissipação, formulado por H. Nyquist [172]. 


\subsection{Método de Monte Carlo (clássico)}

No contexto da Mecânica Estatística, as propriedades de equilíbrio de um sistema são expressas em termos de médias sobre todas as configurações possíveis, regidas pela estatística de Boltzmann. Admite-se como princípio fundamental que a probabilidade $p$ de encontrar o sistema em um estado $s$ qualquer depende de $s$ apenas através de sua energia $E_{s}$, sendo então $p \propto e^{-\beta E_{s}}$, com $\beta=\frac{1}{k_{B} T}$. Para sistemas razoavelmente extensos, essas médias tornam-se impraticáveis de se resolver computacionalmente, sobretudo porque existe uma grande variedade de microestados nos quais o sistema pode se encontrar. Ainda que tentássemos substituir, por exemplo, as médias exatas por médias sobre um número muito menor de estados possíveis, aleatoriamente escolhidos, o cálculo dificilmente seria considerado aplicável em termos práticos. Isso porque, de maneira inevitável, muito do tempo de cálculo seria dedicado à geração de estados com energias elevadas e que pouco contribuiriam na média térmica final.

Assim, o método de Monte Carlo configura-se como uma série de algoritmos dedicados à procura do estado mais provável para o sistema de interesse, segundo determinadas condições fornecidas a priori; trata-se, portanto, de um conjunto de técnicas de amostragem desenvolvidos para os casos nos quais há a influência de variáveis não controladas, mas que podem ser descritas como processos aleatórios. Em particular, Metropolis et al. [173] demonstraram, em 1953, que as configurações possíveis de um dado sistema são mais facilmente encontradas recursivamente, partindo de um estado inicial qualquer. Nesse algoritmo, a alteração, ou não, da configuração atual depende de uma probabilidade de transição que leva em conta a diferença de energia $\Delta E$ entre os estados inicial e final em cada passo da recursão. Note que, nesse sentido, o sucesso do algoritmo de Metropolis é fortemente dependente da geração rápida e eficiente de números aleatórios. Assim, a seguir detalharemos explicitamente o algoritmo de geração de estados, considerando um sistema de spins.

A dinâmica estocástica inicia-se com a escolha de um estado qualquer para o sistema, e de um sítio $i$. Em seguida, calcula-se a diferença de energia $\Delta E=E_{\text {final }}-E_{\text {inicial }}$ resultante a rotação do spin em $i$, considerando que a energia do sistema é representada por uma Hamiltoniana de spin (Eq. 4.4). A esta diferença de energia associa-se um número aleatório $r_{0}\left(0<r_{0}<1\right)$, tal que, se $r_{0}<e^{-\beta \Delta E}$, o estado cujo spin foi rotacionado é assumido nova configuração para a rede de spins. Esse processo é repetido um número grande de vezes, escolhendo-se sempre posições $i^{\prime}$ aleatórias da rede. Cabe ressaltar que, caso a variação de energia seja negativa $(\Delta E<0)$, então, por definição $e^{-\beta \Delta E}>1>r_{0}$ 
e automaticamente a rotação do spin no sítio $i$ é aceita. A Figura 4.2, abaixo, mostra um esquema ilustrativo do esquema de Metropolis. Como o método de Monte Carlo (MC) será utilizado para encontrar a solução de um sistema de spins interagentes entre si via acoplamentos de troca (modelo de Heisenberg, $\mathcal{J}_{i j}$ ) e de DM, a princípio quaisquer rotações dentro da esfera unitária são permitidas.

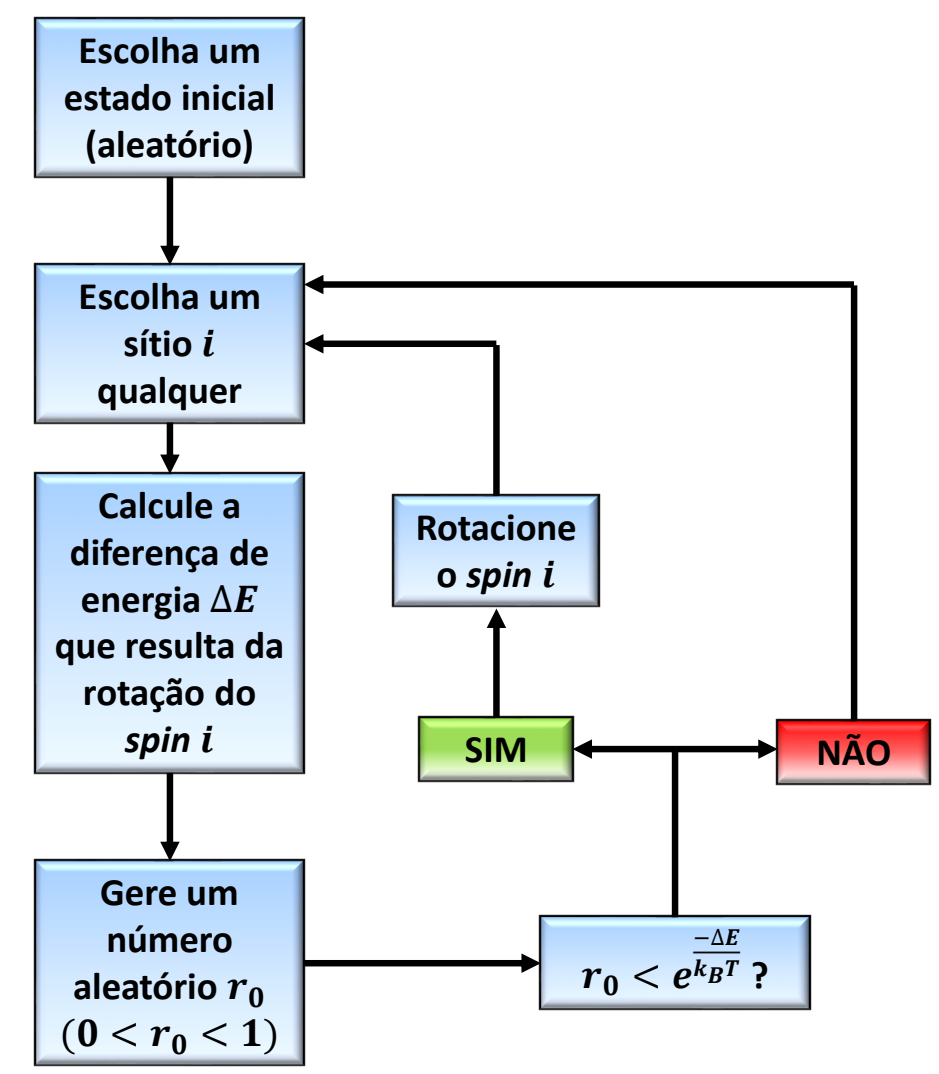

Figura 4.2: Esquema recursivo do algoritmo de Metropolis, aplicado a um sistema de spins. 


\section{Capítulo 5}

\section{Skyrmions em filmes ultrafinos}

\section{$5.1 \mathrm{Pd} / \mathrm{Fe} / \operatorname{Ir}(111)$}

\subsubsection{Preâmbulo}

O sistema organizado em multicamadas de $\mathrm{Pd} / \mathrm{Fe} / \mathrm{Ir}(111)$ foi um dos primeiros - após a observação experimental de skyrmions em cristais contendo metais de transição, tais como o MnSi com simetria cúbica do tipo B20 [42, 174, 175] - a serem reportadas, em baixas temperaturas, estruturas ordenadas (redes) de skyrmions sob a aplicação de um campo magnético externo $[52,55,59,96]$. Experimentalmente, utilizando um microscópio de corrente de tunelamento com spin polarizado (SP-STM) [13], observou-se a formação de uma rede de skyrmions em um campo magnético $\mathbf{B}$ com magnitude entre $1 \mathrm{~T}$ e 2 $\mathrm{T}$, aplicado perpendicularmente ao plano da superfície, à $T=4,2 \mathrm{~K}$ [52]. É relevante notar que os skyrmions observados possuem um tamanho característico de cerca de $3 \mathrm{~nm}$ (embora seu tamanho dependa de $\mathbf{B}[55]$ ), pequenos o suficiente para que possam ser simulados a partir de cálculos de primeiros princípios.

Simultaneamente às pesquisas de caráter experimental, foram conduzidas e publicadas investigações teóricas sobre as propriedades magnéticas do sistema $\mathrm{Pd} / \mathrm{Fe} / \mathrm{Ir}(111)$. Simon et al. [54] encontraram, por exemplo, a existência de fortes interações do tipo DM entre Fe primeiros vizinhos, o que sugeriram ser, em conjunto com a quebra de simetria de inversão, uma das causas da emergência de skyrmions nesse tipo de sistema. Além disso, com o auxílio de simulações baseadas na dinâmica atomística de spins (LLG), determinaram que o comprimento de onda das espirais de spin (estado fundamental da 
multicamada $\mathrm{Pd} / \mathrm{Fe} / \mathrm{Ir}(111)$ ) comporta-se de maneira inversamente proporcional ao aumento da relação $\frac{D}{\mathcal{J}}$ entre primeiros vizinhos Fe-Fe; isso indica uma relação direta entre as interações do tipo DM e o surgimento de configurações não-colineares de spin. Cabe também citar que, pouco tempo depois, Rózsa et al. [53] determinaram o diagrama de fases do sistema $\mathrm{Pd} / \mathrm{Fe} / \mathrm{Ir}(111)$, sugerindo a presença de skyrmions com tempo finito de vida (meta-estáveis) à altas temperaturas $(T \gtrsim 100 \mathrm{~K})$.

Portanto, sendo a multicamada $\mathrm{Pd} / \mathrm{Fe} / \mathrm{Ir}(111)$ um sistema que conhecidamente apresenta uma fase magnética dominada por uma rede de skyrmions, iniciamos nosso estudo por esse sistema. A possibilidade de reproduzir certos resultados já publicados na literatura valida a metodologia aqui utilizada para, posteriormente, aplicarmos à sistemas semelhantes ainda não investigados. Por fim, vale a pena ressaltar que esta é a primeira vez que o método RS-LMTO-ASA é usado diretamente para estudar skyrmions em superfícies magnéticas.

Os cálculos aqui apresentados foram realizados com a implementação computacional de mesmo nome do método (RS-LMTO-ASA), utilizando os recursos computacionais de High Performance Computing da Universidade de São Paulo (HPC-USP, antigo LCCA), do Centro Nacional de Processamento de Alto Desempenho em São Paulo da Universidade Estadual de Campinas (CENAPAD-UNICAMP), do National Supercomputer Centre (NSC-Suécia), e do Laboratório Nacional de Computação Científica (LNCC-MCTI), do qual utilizou-se o supercomputador Santos Dumont.

\subsubsection{Propriedades eletrônicas da bicamada de $\mathrm{Pd} / \mathrm{Fe} / \operatorname{Ir}(111)$}

Devidamente convergidos o nível de Fermi e os parâmetros de potencial do Ir bulk (veja o Apêndice E), partimos então para o modelamento da multicamada $\mathrm{Pd} / \mathrm{Fe} / \mathrm{Ir}(111)$. É importante ressaltar que, nesse sistema, consideramos que o Fe e o $\mathrm{Pd}$ acomodam-se idealmente na estrutura cristalina $f c c$ do Ir, com o mesmo parâmetro de rede de $3,84 \AA$. A superfície foi, então, simulada por um cluster no espaço real contendo $\sim 16.000$ átomos, formada por uma camada de esferas vazias, uma monocamada de Pd, uma monocamada de Fe, e 5 camadas não-equivalentes de Ir, nesta ordem; para as demais camadas de Ir, os parâmetros de potencial foram fixados naqueles obtidos para o bulk. O critério adotado para a quantidade de camadas não-equivalentes de Ir constituiu-se na comparação entre as ocupações eletrônicas para um átomo típico da última camada calculada com as obtidas para o Ir bulk. Caso essa diferença seja da ordem de $10^{-3}$ elétron para cada orbital $s$, $p$ ou $d$ da base local, então consideramos que a densidade eletrônica da superfície está 
convergida, sendo que as perturbações emergentes das camadas com elementos distintos (Fe e Pd) são progressivamente suprimidas com o aumento da distância. A Figura 5.1, a seguir, apresenta as LDOS de um átomo típico das camadas de $\mathrm{Pd}, \mathrm{Fe}, \mathrm{Ir}_{1}$ e $\mathrm{Ir}_{5}$, onde $\operatorname{Ir}_{n}$ simboliza a $n$-ésima camada de Ir após a monocamada de Fe, bem como uma comparação entre as LDOS totais obtidas para o $\operatorname{Ir}_{5}$ e para o Ir bulk. 

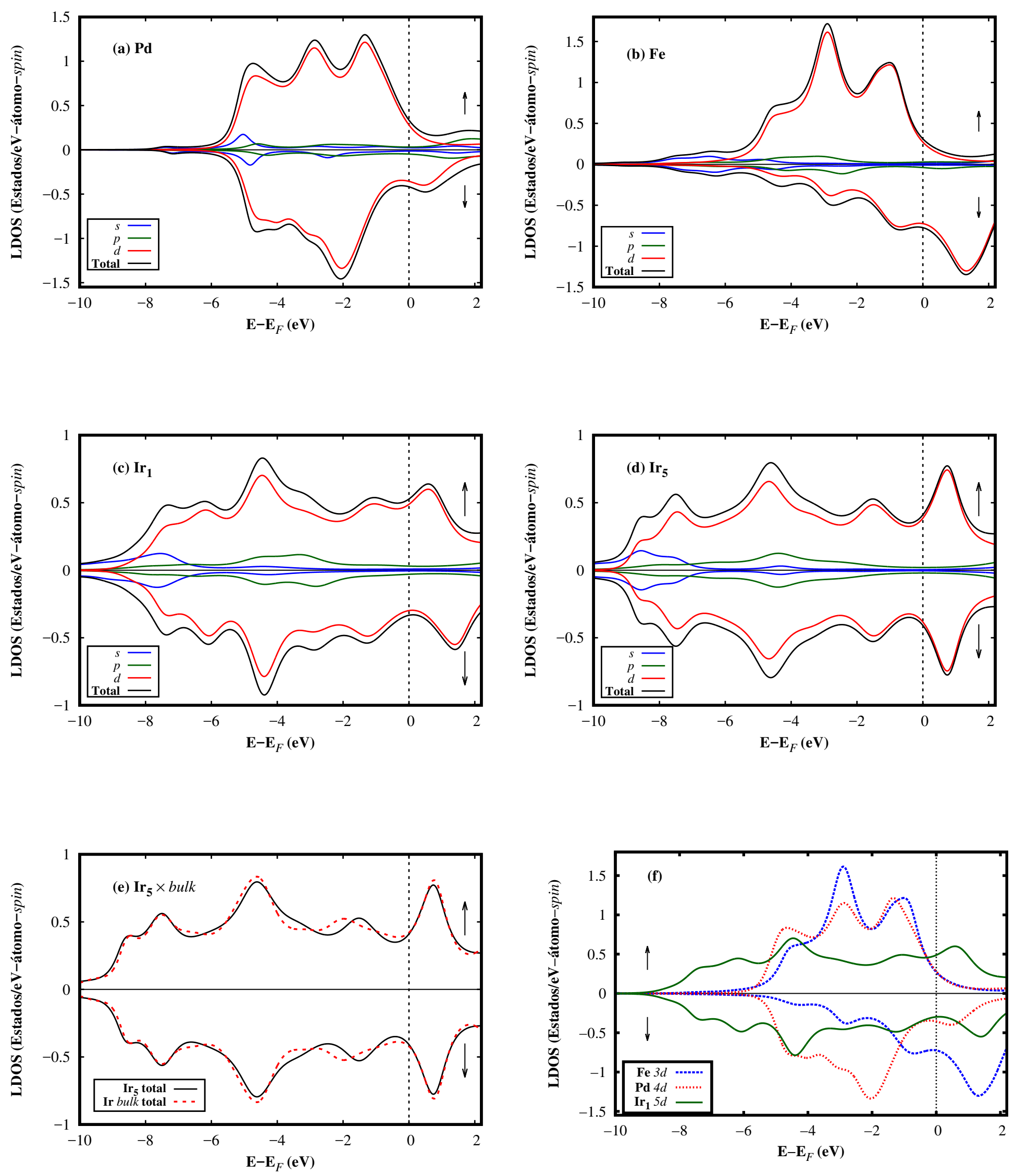

Figura 5.1: LDOS totais e projetadas nos obitais $s, p, d$ para a superfície de $\mathrm{Pd} / \mathrm{Fe} / \mathrm{Ir}(111)$, para um sítio na: (a) camada de $\mathrm{Pd} ;(b)$ camada de $\mathrm{Fe}$; $(c)$ primeira camada de Ir $\left(I r_{1}\right) ;(d)$ quinta camada de Ir $\left(I r_{5}\right) ;(e)$ comparação entre as LDOS totais da última camada de Ir não-equivalente e de um átomo típico do Ir bulk $(f c c)$; $(f)$ comparação entre as LDOS para os orbitais $d$ de átomos típicos das camadas de $\mathrm{Pd}, \mathrm{Fe}$, e $\mathrm{Ir}_{1}$. As setas $\uparrow \mathrm{e} \downarrow$ indicam as ocupações por spin nas bandas majoritária e minoritária, respectivamente. $E_{F}$ é a energia de Fermi do sistema $\left(=E_{F}\right.$ para o Ir bulk). 
Verifica-se que a contribuição dominante para as LDOS advém dos estados $d$ para todos os elementos (Fe, Pd, Ir), como é de se esperar para metais de transição. Por sua vez, as assimetrias entre as densidades de estado para as bandas majoritárias ( $\uparrow$ ) e minoritárias $(\downarrow)$ dos átomos típicos das camadas de $\mathrm{Fe}, \mathrm{Pd}$, e $\mathrm{Ir}_{1}$, claramente observadas nas Figs. 5.1(a)-(c), resultam em diferentes ocupações eletrônicas locais, e estão relacionadas à emergência de momentos magnéticos de spin locais não-nulos nessas camadas. A convergência da densidade eletrônica da superfície de $\mathrm{Pd} / \mathrm{Fe} / \mathrm{Ir}(111)$ é justificada pela Fig. 5.1(e), na qual se nota que as LDOS para a última camada não-equivalente de Ir (Fig. 5.1(d)) e para o Ir bulk apresentam apenas pequenas diferenças. Por fim, a Fig. 5.1(f) mostra que há uma grande faixa de energia na qual as LDOS do Pd e do Fe se sobrepõem, especialmente entre os estados majoritários de spin, indicando uma elevada hibridização entre os orbitais $3 d-4 d$ de ambos. Além disso, nota-se que os estados $5 d$ do Ir estendemse por um maior intervalo de energia. Embora o Ir apresente uma densidade local de estados não-desprezível no nível de Fermi (Fig. 5.1(f)), o que levaria, em princípio, a um maior produto de Stoner, a redistribuição de carga devido à associação à camada de Fe é pequena quando comparada à configuração eletrônica do Ir bulk $(f c c)$, gerando também um pequeno momento magnético de spin induzido nesse material. Como as bandas $s$ e $p$ não contêm uma parte expressiva da carga de valência para os sítios típicos nas camadas de Pd, Fe, e Ir, essas não foram consideradas na presente análise.

Os cálculos para diferentes configurações magnéticas colineares da superfície de $\mathrm{Pd} / \mathrm{Fe}$ depositado em $\operatorname{Ir}(111)$, incluindo o acoplamento spin-órbita na autoconsistência, indicam, em princípio, que o estado fundamental do sistema é atingido quando os spins do Fe estão todos alinhados à uma mesma direção de magnetização (estado ferromagnético, FM). Nessa configuração, os momentos magnéticos de $\operatorname{spin}\left(m_{S}\right)$ e orbitais $\left(m_{L}\right)$ obtidos para cada átomo típico do sistema $\mathrm{Pd} / \mathrm{Fe} / \mathrm{Ir}(111)$ estão resumidos na Tabela 5.1. É interessante observar que o valor de $m_{S}$ calculado para o Pd é relativamente grande, demonstrando a elevada polarizabilidade magnética desse material. Esse foi justamente o motivo pelo qual Romming et al. [52] decidiram incluir a camada de $\mathrm{Pd}$ no sistema de $\mathrm{Fe} / \mathrm{Ir}(111)$ : até então, skyrmions espontâneos haviam sido observados experimentalmente na monocamada de Fe/Ir(111) [46], porém o controle de criação/aniquilação dessas configurações topológicas só poderia ocorrer em um sistema cujo estado fundamental não fosse composto por uma rede de skyrmions, e sim os estados excitados. Assim, os autores da referência [52] incluíram o $\mathrm{Pd}$ no topo do Fe, pois, devido à sua elevada polarizabilidade magnética [176], argumentaram que a bicamada PdFe se comportaria como uma única entidade magnética. Por outro lado, os momentos induzidos no Ir são baixos e preferem um alinhamento antiparalelo à magnetização do Fe. Como esperado, encontramos que, quanto maior a distância entre as camadas de Fe e Ir, mais os momentos magnéticos de 
spin aproximam-se de zero. Entretanto, cabe ressaltar que, a partir da terceira camada de Ir, os momentos de spin tendem a ordenar-se paralelamente à $m_{S}^{\mathrm{Fe}}$, exibindo, portanto, uma oscilação do alinhamento entre os momentos de spin. Esse mesmo tipo de comportamento já havia sido observado para o sistema similares, como o de Fe/W(001) [177] por exemplo, e pode ser entendido como uma consequência da hibridização entre os estados $3 d-5 d$ e, em menor extensão, do efeito geométrico de se haver uma única interface no sistema. Para todos os tipos atômicos, os momentos magnéticos orbitais $\left(m_{L}\right)$ são bastante reduzidos, da ordem de $10^{-2} \mu_{B}$ /átomo (veja Tabela 5.1), ressaltando que as propriedades magnéticas e dinâmicas da multicamada $\mathrm{Pd} / \mathrm{Fe} / \mathrm{Ir}(111)$ são majoritariamente decorrentes dos spins.

Tabela 5.1: Momentos magnéticos de $\operatorname{spin}\left(m_{S}\right)$ e orbitais $\left(m_{L}\right)$ calculados para um átomo típico de cada camada do sistema $\mathrm{Pd} / \mathrm{Fe} / \mathrm{Ir}(111)$. Os valores são dados em $\mu_{B} /$ átomo.

\begin{tabular}{|c|c|c|}
\hline Camada & $\mathbf{m}_{\mathbf{S}}$ & $\mathbf{m}_{\mathbf{L}}$ \\
\hline $\mathbf{P d}$ & 0,31 & 0,02 \\
\hline $\mathbf{F e}$ & 2,69 & 0,09 \\
\hline $\mathbf{I r}_{1}$ & $-0,04$ & $-0,04$ \\
\hline $\mathbf{I r}_{2}$ & $-0,05$ & $<10^{-2}$ \\
\hline $\mathbf{I r}_{3}$ & $<10^{-2}$ & $<10^{-2}$ \\
\hline $\mathbf{I r}_{4}$ & $<10^{-2}$ & $<10^{-2}$ \\
\hline $\mathbf{I r}_{5}$ & $<10^{-2}$ & $<10^{-2}$ \\
\hline
\end{tabular}

O processo autoconsistente para a simulação de sistemas metálicos bidimensionais (superfícies) implementado no código RS-LMTO-ASA considera que, para cada camada não-equivalente incluída no cálculo, os átomos pertencentes àquela camada têm todos as mesmas propriedades eletrônicas locais, e as transferências de carga dependem apenas da interação entre cada camada. Isso significa que para a convergência da estrutura eletrônica do sistema $\mathrm{Pd} / \mathrm{Fe} / \mathrm{Ir}(111)$ houve a necessidade de se considerar 8 (oito) sítios atômicos distintos na autoconsistência (veja Tabela 5.1), levando também em conta um átomo com carga de valência nula (esfera vazia) para simular os efeitos de vácuo no material (veja a seção 3.2.3). Logo, as interações mistas (Fe-Pd, Ir-Pd, Fe-Ir) mais distantes que primeiros vizinhos, e os acoplamentos entre átomos de mesmo elemento (Fe-Fe, Pd-Pd, Ir-Ir) em princípio não poderiam ser considerados no cálculo inicial.

A fim de contornar esse problema, recalculamos ilhas de átomos da superfície como impurezas inseridas na própria multicamada $\mathrm{Pd} / \mathrm{Fe} / \mathrm{Ir}(111)$, de forma a considerar sua presença como perturbações locais ao hospedeiro [134], e obter as propriedades eletrônicas e magnéticas átomo-a-átomo. Para a construção de cada ilha, seguiu-se o critério de, a 
partir do átomo de Fe mais centralizado possível (para evitar efeitos de borda no aglomerado convergido), tomar o aglomerado formado pelos átomos tal que sua distância $d$ ao Fe central fosse menor ou igual a uma distância $d_{\text {máx }}$ previamente definida $\left(d \leq d_{\text {máx }}\right)$. Portanto, a ilha de primeiros vizinhos seguiu o vínculo $d \leq a \frac{\sqrt{2}}{2} \sim 2,72 \AA$. Já a ilha de segundos vizinhos foi formada pelos átomos da superfície tal que $d \leq a=3,84 \AA$, e assim por diante. Repetimos esse procedimentos e calculamos a estrutura eletrônica para ilhas até décimos-quartos vizinhos (no bulk), nos quais $d_{\text {máx }}=a \sqrt{6} \sim 9,41 \AA$. Uma representação esquemática das ilhas para primeiros vizinhos do átomo de Fe central (um cluster contendo 13 átomos) e para décimos-quartos vizinhos do mesmo sítio de referência (um cluster contendo 188 átomos não-equivalentes) pode ser visualizada na Figura 5.2, abaixo.

(a)

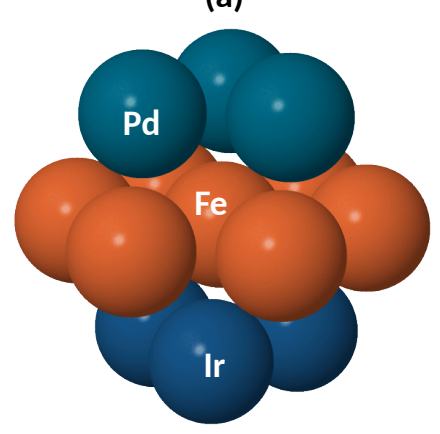

(b)

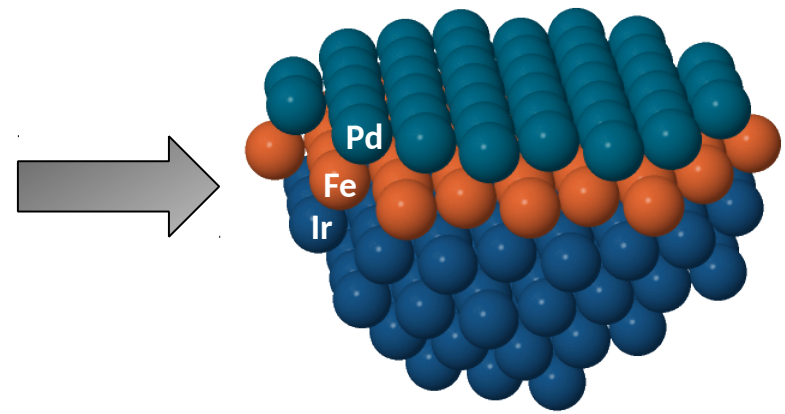

Figura 5.2: Representação esquemática das ilhas (aglomerados) de átomos recalculados autoconsistentemente como impurezas na multicamada $\mathrm{Pd} / \mathrm{Fe} / \operatorname{Ir}(111)$, considerando cada sítio como não-equivalente: $(a)$ primeiros vizinhos $\left(d_{\text {máx }}=2,72 \AA\right)$; e $(b)$ décimos-quartos vizinhos $\left(d_{\text {máx }}=9,41 \AA\right)$ do átomo de Fe central.

Na Figura 5.3 são apresentados os valores das interações de troca $\left(\mathcal{J}_{i j}\right)$ entre pares de átomos de Fe localizados em uma mesma camada para o cálculo considerando os spins alinhados a uma mesma direção de magnetização (cálculo colinear). Como se pode notar, os valores de $\mathcal{J}_{\mathrm{Fe}-\mathrm{Fe}}$, para a terceira e quarta vizinhanças, tornam-se negativos, indicando uma preferência pelo acoplamento antiferromagnético (AFM) entre os momentos de spin dos átomos de Fe. De fato, definindo a contribuição efetiva das interações de troca para um determinado sítio atômico $i$ como $\mathcal{J}_{i j}^{\text {eff }}=\sum_{j} \mathcal{J}_{i j}$, temos que $\mathcal{J}_{\mathrm{Fe}-\mathrm{Fe}}^{\text {eff }}=147,03 \mathrm{meV}$ para os acoplamentos com a primeira vizinhança de $\mathrm{Fe}$, e $\mathcal{J}_{\mathrm{Fe}-\mathrm{Fe}}^{\text {eff }}=-23,37 \mathrm{meV}$ para a terceira vizinhança, demonstrando uma contribuição não-desprezível dos valores de $\mathcal{J}_{i j}$ para interações com terceiros vizinhos Fe-Fe $(\sim 16 \%)$. Tratam-se, portanto, de interações de longo alcance, já que os terceiros vizinhos de Fe em uma mesma camada localizam-se $\mathrm{a} \sim 5,43 \AA$ A do sítio de referência. Por outro lado, a presença não-desprezível de valores de $\mathcal{J}_{\mathrm{Fe}-\mathrm{Fe}}$ negativos indica uma certa frustração magnética entre distintas camadas de 
vizinhos.

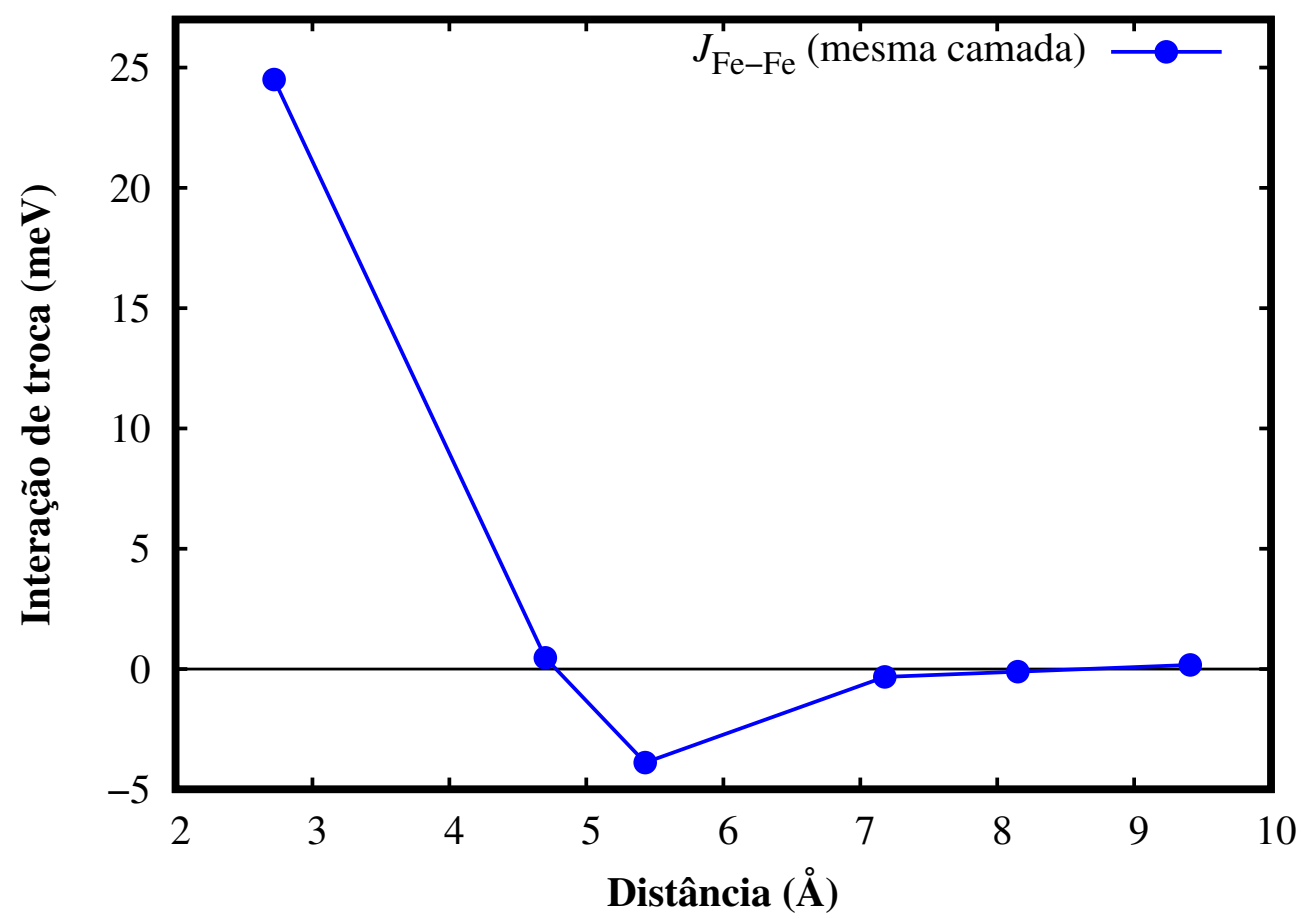

Figura 5.3: Valores das interações de troca entre pares de átomos Fe-Fe localizados na mesma camada $\left(\mathcal{J}_{\mathrm{Fe}-\mathrm{Fe}}\right)$, em função da distância entre os átomos do par.

Os resultados apresentados na Fig. 5.3 não levam em consideração possíveis relaxações entre as camadas do sistema $\mathrm{Pd} / \mathrm{Fe} / \operatorname{Ir}(111)$, e, portanto, os valores de $\mathcal{J}_{i j}$ obtidos são maiores do que os previamente reportados [54,60]. Entretanto, o forte caráter FM para as interações de primeiros vizinhos Fe-Fe, obtido nas referências [54, 60], também é observado para o sistema ideal, isto é, sem considerar a otimização estrutural. Realizamos também cálculos para a superfície de $\mathrm{Pd} / \mathrm{Fe} / \mathrm{Ir}(111)$, utilizando o método de pseudopotenciais Projector Augmented Wave (PAW) [178, 179], tal como implementado no pacote computacional Quantum ESPRESSO [180, 181], sugerem as seguintes relaxações para as camadas, à pressão nula: 3,04\% para o $\mathrm{Pd}$, em direção à camada adjacente de $\mathrm{Fe}$, abaixo; 2,31\% para o Fe, em direção à camada adjacente de Ir, abaixo; e 0,85\% para o Ir, em direção à camada de Fe, acima. As diferenças verificadas para as distâncias interatômicas em relação ao sistema ideal ocorreram unicamente no eixo- $z$, mantendo a simetria original $C_{3 h}$ de cada plano da superfície.

Considerando as interações de troca entre camadas (Fe-Pd, Fe-Ir, e Pd-Ir), vale a pena mencionar o acoplamento não-desprezível entre primeiros vizinhos de $\mathrm{Fe}-\mathrm{Pd}$, com $\mathcal{J}_{\mathrm{Fe}-\mathrm{Pd}}=3,00 \mathrm{meV}$, o que sugere uma forte interação entre essas duas camadas adjacentes. Esse resultado corrobora com o fato de haver uma significativa hibridização entre 
os orbitais $4 d-3 d$ dos átomos de $\mathrm{Pd}$ e Fe, respectivamente (veja a Fig. 5.1(f)). O restante dos valores encontrados é pequeno, e não devem exercer significativa influência nas propriedades magnéticas da multicamada $\mathrm{Pd} / \mathrm{Fe} / \mathrm{Ir}(111)$.

Tratando, agora, das interações anisotrópicas do tipo Dzyaloshinskii-Moriya (DM) $[48,49]$, as quais possuem um papel signficativo na formação de texturas de spin complexas em sistemas bidimensionais (não-colineares) [50], encontramos que as interações entre os pares Fe-Fe são as mais proeminentes para o sistema $\mathrm{Pd} / \mathrm{Fe} / \mathrm{Ir}(111)$. Esse resultado era de certa forma esperado, já que podemos entender a interação de DM como uma consequência do hopping de elétrons de condução que estão sujeitos a um espalhamento dependente do acoplamento spin-órbita, em um campo cristalino sem simetria de inversão [50]. Esse efeito é conhecido e foi bem descrito teoricamente por Smith [182] e Fert [183]. Nesse caso, a forte interação $\mathcal{J}_{\mathrm{Fe}-\mathrm{Fe}}$ entre primeiros vizinhos de Fe (Fig. 5.3) demonstra que os elétrons deste elemento são os que apresentam maior probabilidade de hopping entre orbitais de sítios adjacentes, e que, consequentemente, sofrerão maior influência do elevado acoplamento spin-órbita do Ir. Por outro lado, a presença de estados estendidos do Ir (orbitais $5 d$ ) favorece o surgimento de interações de mais longas distâncias [34], tal como foi verificado para as interações de troca (isotrópicas), e como se espera nas interações anisotrópicas de DM.

A Figura 5.4(a) mostra o valor médio do módulo do vetor de DM $\left(\left|\mathbf{D}_{\mathrm{Fe}-\mathrm{Fe}}\right|\right)$ entre pares de átomos Fe-Fe (mesma camada), em função da distância interatômica. Nota-se que, apesar de existir uma diminuição do valor de $\left|\mathbf{D}_{\mathrm{Fe}-\mathrm{Fe}}\right|$ com o aumento da distância, há ainda interações expressivas para grandes separações (da ordem de $20 \%$ do acoplamento entre primeiros vizinhos), sobretudo para a quinta vizinhança, a uma distância de 8,15 Å do átomo de referência. Em comparação com os resultados publicados na literatura, encontramos um valor de $\left|\mathbf{D}_{\mathrm{Fe}-\mathrm{Fe}}\right|=0,87 \mathrm{meV}$ entre primeiros vizinhos Fe-Fe, que está em bom acordo com estudos anteriores $\left(\left|\mathbf{D}_{\mathrm{Fe}-\mathrm{Fe}}\right|=0,82 \mathrm{meV}\right.$ para uma relaxação de $5 \%$ na camada de Fe em direção à superfície de $\operatorname{Ir}(111)$ [54] e $\left|\mathbf{D}_{\mathrm{Fe}-\mathrm{Fe}}\right|=1,0 \mathrm{meV}$ para o sistema $\mathrm{Pd} / \mathrm{Fe} / \mathrm{Ir}(111)$ ideal [60]), considerando as diferentes abordagens teóricas utilizadas. 

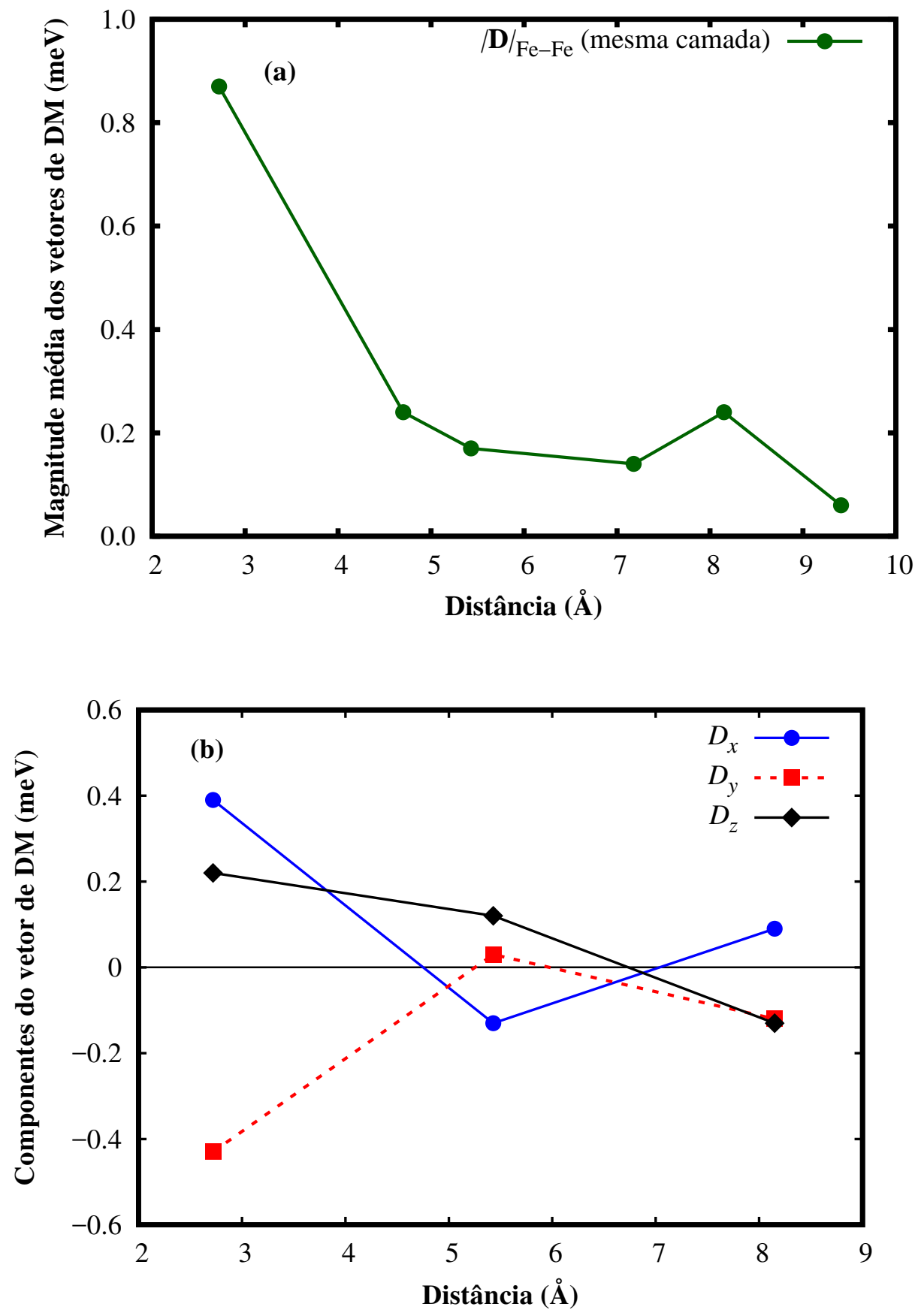

Figura 5.4: Resultados para: (a) valores médios das magnitudes $\left|\mathbf{D}_{\mathrm{Fe}-\mathrm{Fe}}\right|$ entre pares de átomos Fe-Fe, em função da distância interatômica; (b) componentes $D_{x}, D_{y}$, e $D_{z}$ para a interação Fe-Fe entre vizinhos, na direção [101], em função da distância interatômica.

Antes de prosseguirmos, porém, é importante recapitular que a convenção utilizada neste trabalho é a de que o termo do Hamiltoniano de spin para ambas as interações de troca e de DM já incluem em seus valores os momentos magnéticos, o restante da expressão depende apenas dos versores $\hat{\mathbf{e}}_{i, j}$ paralelos à direção local de magnetização no 
sítio $i, j[34,77]$ :

$$
\mathcal{H}_{\text {spin }}=-\sum_{(i, j)} \mathcal{J}_{i j} \hat{\mathbf{e}}_{i} \cdot \hat{\mathbf{e}}_{j}-\sum_{(i, j)} \mathbf{D}_{i j} \cdot\left[\hat{\mathbf{e}}_{i} \times \hat{\mathbf{e}}_{j}\right]
$$

Em outras palavras, as magnitudes dos momentos locais foram incorporadas às constantes de troca $\mathcal{J}_{i j}$ e aos módulos dos vetores $\left|\mathbf{D}_{i j}\right|$ que caracterizam as interações magnéticas entre os sítios atômicos. Assim, quando nos referimos ao valor de $\mathcal{J}_{i j}$ ou de $\left|\mathbf{D}_{i j}\right|$ estamos, de fato, especificando o próprio termo de energia da respectiva interação.

Por sua vez, a Figura 5.4(b) mostra a variação das componentes $D_{x}, D_{y}$ (ambas no plano) e $D_{z}$ (fora do plano) do vetor $\mathbf{D}_{\mathrm{Fe}-\mathrm{Fe}}=\left(D_{x}, D_{y}, D_{z}\right)$, considerando a direção [101], para diferentes distâncias interatômicas. Verifica-se que as compoentes no plano (especialmente $D_{x}$ ) exibem um comportamento oscilatório. O mesmo tipo de comportamento foi recentemente reportado por Khajetoorians et al. [184] para adátomos de Fe em Pt(111), e também por Simon et al. [54], para a multicamada $\mathrm{Pd} / \mathrm{Fe} / \mathrm{Ir}(111)$ considerando relaxações estruturais. Esse comportamento oscilatório, somado ao fato de que existe uma contribuição não-desprezível da quinta vizinhança para as interações de DM, demonstra a natureza de longo alcance dos acoplamentos anisotrópicos, mediados pelo hopping de elétrons através, principalmente, dos estados $5 d$ estendidos do Ir. O forte acoplamento de DM observado na Fig. $\mathbf{5 . 4}$ indica que há condições favoráveis para a emergência de estruturas complexas (não-colineares) de spin no estado fundamental - diferentemente do que se conclui com base apenas em cálculos colineares (muito embora seja possível verificar uma clara frustração magnética).

Com os valores calculados das interações de troca e de DM entre pares de átomos Fe-Fe (mesma camada), é possível obter também a relação $\frac{|\mathbf{D}|}{J}$ para primeiros vizinhos, que tem sido bastante discutida na literatura como de importância fundamental para a formação de skyrmions sob condições favoráveis [54, 91]. Para o sistema ideal de Pd/Fe/Ir(111) encontramos $\frac{|\mathbf{D}|}{J} \sim 0,036$, que está de acordo com a referência [54], dada a tendência encontrada pelos autores de aumento da quantidade $\frac{|\mathbf{D}|}{J}$ conforme a camada de Fe é aproximada da camada adjacente de Ir. O aumento dessa relação também está diretamente relacionado à diminuição do comprimento de onda $\lambda$ da espiral de spin que caracteriza o estado fundamental da multicamada $\mathrm{Pd} / \mathrm{Fe} / \mathrm{Ir}(111)$.

Uma recente investigação experimental sobre a multicamada $\mathrm{Pd} / \mathrm{Fe} / \mathrm{Ir}(111)$ [55] encontrou que a constante de interação $D$ de DM para esse sistema tem o valor $D=(3,9 \pm 0,2)$ $\mathrm{mJ} / \mathrm{m}^{2}$, expressa como uma densidade de energia. Uma sugestão para tentar compa- 
rar esse resultado aos cálculos de primeiros princípios desenvolvidos no presente trabalho é a seguinte. Cada átomo de Fe dentro da mesma camada está sujeito à interações anisotrópicas de DM com todos os seus átomos de Fe vizinhos (consideraremos os acoplamentos mistos Fe-Pd e Fe-Ir como desprezíveis). Portanto, é possível definir uma magnitude efetiva para os vetores de DM $D^{\text {eff }}$ tal que incorpore todas as contribuições das interações do tipo DM entre pares Fe-Fe: $D^{\text {eff }}=\sum_{i}\left|\mathbf{D}_{1 i}\right|$, onde "1" representa o sítio atômico de referência. Se examinarmos apenas as interações entre primeiros vizinhos (responsáveis pela maior contribuição à $D^{\mathrm{eff}}$ ), a vizinhança resultante tem uma forma hexagonal (simetria $C_{3 h}$ ), todos à mesma distância de $\sim 2,72 \AA$ do átomo de Fe central, como pode ser visto na Figura 5.5, a seguir. Assim, se obtém uma magnitude efetiva para os acoplamentos de $\mathrm{DM}$ de $D^{\mathrm{eff}}=5,21 \mathrm{meV}$. A lista completa das componentes de cada interação, seguindo a numeração definida na Fig. 5.5, é apresentada na Tabela 5.2. O acoplamento efetivo de DM resulta em uma densidade $D$ de

$$
D=\frac{D^{\mathrm{eff}}}{\left(\frac{3 l^{2} \sqrt{3}}{2}\right)}=\frac{5,21 \mathrm{meV}}{19,16 \AA^{2}} \sim 4,36 \mathrm{~mJ} / \mathrm{m}^{2}
$$

onde $l=a \frac{\sqrt{2}}{2}=2,72 \AA$. Assim, o valor obtido está em bom acordo com o resultado experimental (inclusive dentro de três incertezas), considerando todas as aproximações envolvidas nessa comparação. Vale a pena também destacar que esse método é similar ao realizado pelos próprios autores da referência [55] (veja o Material Suplementar dessa referência), para a obtenção, a partir de cálculos ab initio, a saturação de magnetização do material - na qual foi assumida uma distribuição uniforme do momento magnético local para a bicamada de PdFe (onde $m_{S}^{\text {total }}=m_{S}^{\mathrm{Fe}}+m_{S}^{\mathrm{Pd}}$ ).

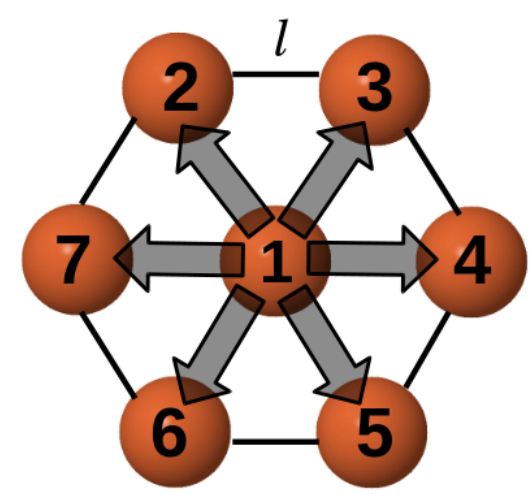

Figura 5.5: Estrutura de primeiros vizinhos de um átomo de Fe ("1") em uma mesma camada, todos a uma distância $l=a \frac{\sqrt{2}}{2}=2,72 \AA$ do átomo de referência. As esferas laranjas representam átomos de $\mathrm{Fe}$, e as setas as interações consideradas. 
Tabela 5.2: Componentes dos vetores de DM $\left(\mathbf{D}_{\mathrm{Fe}-\mathrm{Fe}}\right)$, expressos em meV, para as interações entre primeiros vizinhos de pares Fe-Fe. A numeração segue a definição da Fig. 5.5 .

\begin{tabular}{rrrr}
\hline$(i, j)$ & \multicolumn{1}{c}{$D_{x}$} & \multicolumn{1}{c}{$D_{y}$} & \multicolumn{1}{c}{$D_{z}$} \\
\hline \hline$(1,2)$ & $-0,48$ & $-0,48$ & 1,19 \\
$(1,3)$ & 0,39 & $-0,43$ & 0,22 \\
$(1,4)$ & 0,43 & $-0,39$ & $-0,22$ \\
$(1,5)$ & 0,48 & 0,48 & $-1,19$ \\
$(1,6)$ & $-0,39$ & 0,43 & $-0,22$ \\
$(1,7)$ & $-0,43$ & 0,39 & 0,22 \\
\hline
\end{tabular}

Uma inspeção minuciosa das componentes apresentadas na Tabela $\mathbf{5 . 2}$ nos permite verificar a equivalência entre os pares Fe-Fe opostos $(1,2) \leftrightarrow-(1,5),(1,3) \leftrightarrow-(1,6)$, e $(1,4) \leftrightarrow-(1,7)$ (numerações definidas na Fig. 5.5), tal como esperada para o grupo de simetria $C_{3 h}$ da monocamada de Fe. Resultados similares foram encontrados na referência [54]. É relevante notar que os vetores de DM, como pode ser observado, são quase paralelos ao plano da superfície. De fato, se calcularmos o ângulo médio entre os vetores $\mathbf{D}_{\mathrm{Fe}-\mathrm{Fe}}$ para primeiros vizinhos e o plano da superfície de $\operatorname{Ir}(111)$, definido aqui como $\bar{\phi}$, encontramos um pequeno valor de apenas $\bar{\phi} \sim 8^{\circ}$. Por outro lado, as interações com vizinhos de Fe mais distantes apresentam um comportamento completamente distinto. Ao invés de vetores $\mathbf{D}_{\mathrm{Fe}-\mathrm{Fe}}$ sempre quase paralelos ao plano, em alguns casos os vetores de DM encontramse mais próximos da direção perpendicular ao plano ([111]), com ângulos $\bar{\phi}$ que variam de $\bar{\phi} \sim 65^{\circ}$ (na segunda vizinhança) a $\bar{\phi} \sim 46^{\circ}$ (na sexta vizinhança), enquanto outros permanecem próximos ao plano de superfície. Entretanto, em todos os casos investigados se nota a equivalência esperada por simetria, tal qual verificada para as interações de primeiros vizinhos Fe-Fe. Uma representação esquemática dos vetores de DM (apenas as direções) obtidos via cálculos de primeiros princípios para primeiros e segundos vizinhos de um átomo de $\mathrm{Fe}$ na multicamada $\mathrm{Pd} / \mathrm{Fe} / \mathrm{Ir}(111)$ é apresentada na Figura 5.6. Vale a pena ressaltar que, embora os vetores $\mathbf{D}_{\mathrm{Fe}-\mathrm{Fe}}$ estejam relacionados à interações de pares, na Figura 5.6 eles estão representados no sítio do segundo átomo do par - assumindo o primeiro átomo como sendo, em todos os casos, o Fe central. 


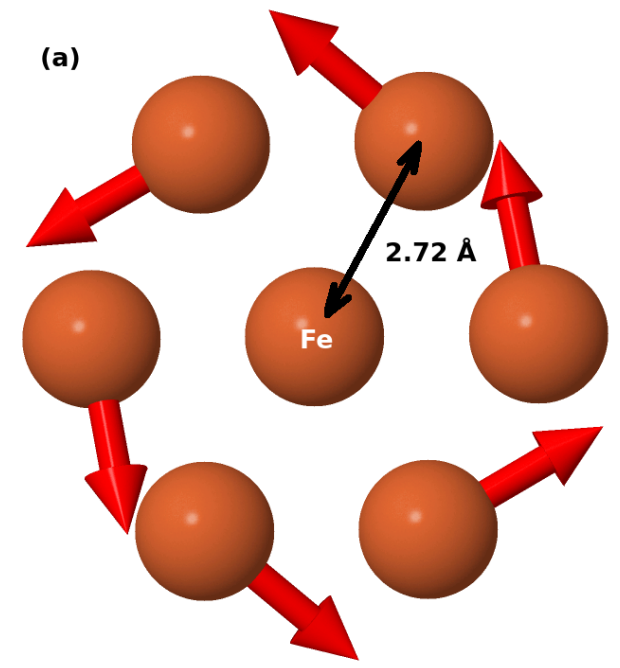

(b)

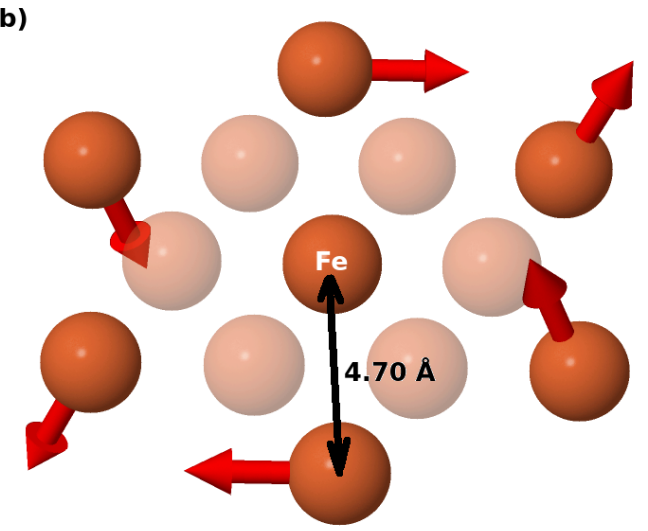

Figura 5.6: Direções calculadas dos vetores $\mathbf{D}_{\mathrm{Fe}-\mathrm{Fe}}$ (em vermelho) para interações de: (a) primeiros vizinhos; (b) segundos vizinhos com o átomo de Fe central (indicado). As esferas em laranja representam os átomos de Fe.

Note que, embora T. Moriya [49] tenha estabelecido algumas regras para se determinar a direção de $\mathbf{D}_{i j}$ com base em argumentos de simetria, A. Crèpieux e C. Lacroix [185] demonstraram minuciosamente que essas regras não são, de fato, suficientes para todo tipo de sistema - dentre eles, as superfícies do tipo $f c c(111)$. Assim, para o caso de spins $\mathbf{S}_{i}$ e $\mathbf{S}_{j}$ primeiros vizinhos, localizados em uma mesma camada de superfície $f c c(111)$, não é possível dizer, apenas pela simetria do sistema, que alguma das componentes de $\mathbf{D}_{i j}$ desaparece, sendo necessárias simulações caso-a-caso a fim de se determinar com certeza a direção do vetor de DM.

A competição entre as interações de troca, para as quais, dependendo da distância, há uma preferência pela configuração FM ou pelo alinhamento AFM dos momentos magnéticos de spin, além das fortes interações de DM encontradas (sobretudo para pares de primeiros vizinhos $\mathrm{Fe}-\mathrm{Fe}$ ), sugerem que a configuração magnética do estado fundamental para o sistema $\mathrm{Pd} / \mathrm{Fe} / \mathrm{Ir}(111)$ deve afastar-se do arranjo colinear (FM) obtido inicialmente, o que de fato ocorre. A fim de analisar essa possibilidade diretamente por métodos ab initio, realizamos cálculos que permitem a obtenção de arranjos nãocolineares de spin. Assim, considerando o maior aglomerado de átomos não-equivalentes (Fig. 5.2(b)), os resultados finais para as camadas de Pd e Fe, considerando o efeito do acoplamento spin-órbita no cálculo autoconsistente, são apresentados na Figura 5.7. 

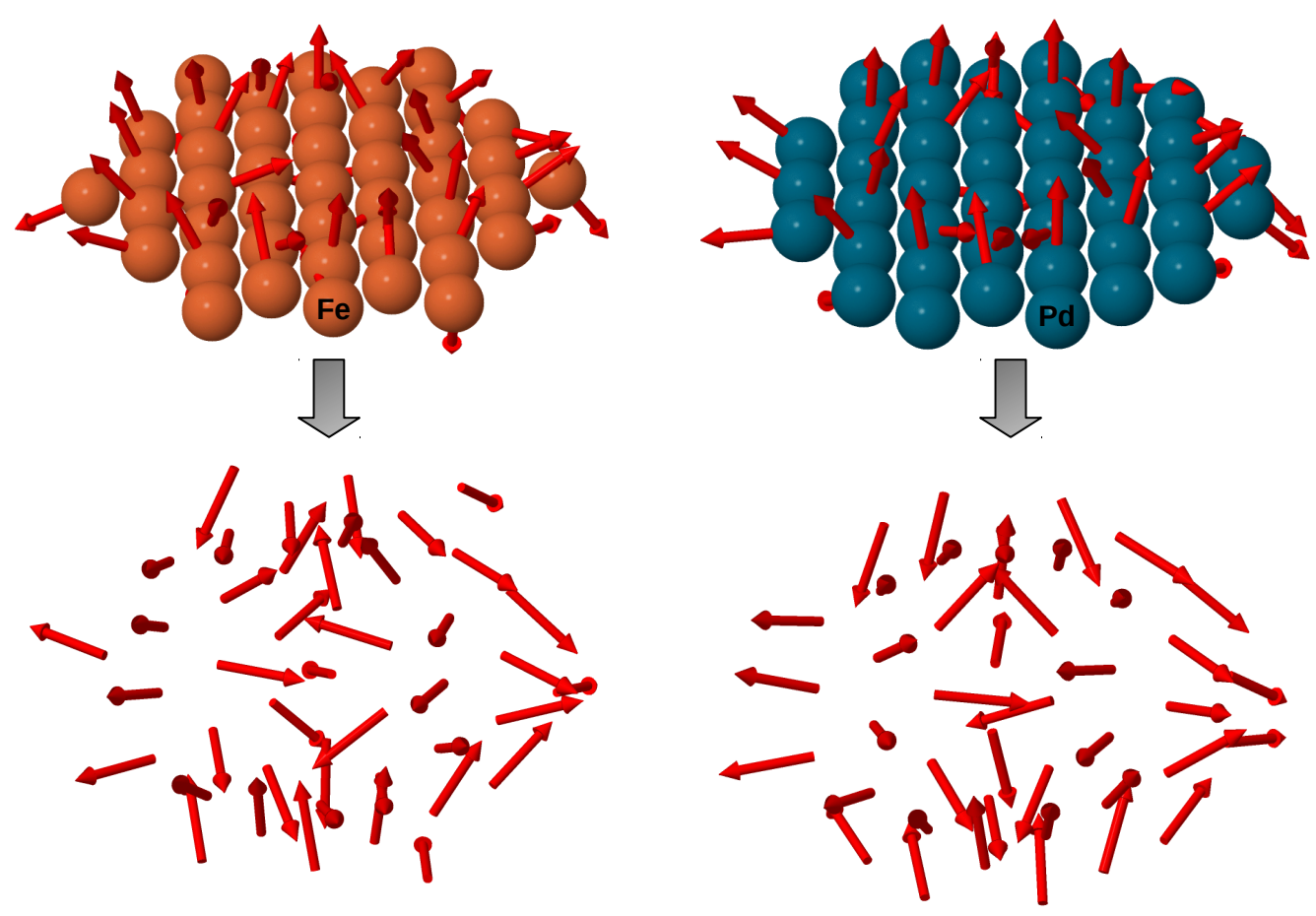

Figura 5.7: Direções obtidas para os momentos de spin (em vermelho) atômicos nas camadas de $\mathrm{Pd}$ e Fe, considerando o cálculo para o aglomerado de átomos não-equivalentes representado na Fig. 5.2(b). As esferas laranjas representam átomos de Fe, enquanto que as esferas azuis representam átomos de $\mathrm{Pd}$.

É interessante notar, por meio de simples inspeção visual, que a não-colinearidade dos spins na camada de Pd segue a tendência observada para a camada de Fe. A Tabela 5.3 contém os ângulos $\theta_{n}$ entre os $n$ momentos magnéticos de spin contidos em uma mesma vizinhança com relação ao átomo central, tanto para a camada de Fe quanto para a camada de Pd. A proximidade entre os valores encontrados atesta quantitativamente que, de fato, há grande semelhança entre as texturas de spin obtidas para ambas as camadas. Isso é um reflexo direto do forte acoplamento de troca entre as camadas de Fe-Pd, bem como da acentuada hibridização dos estados $4 d-3 d$ entre as duas espécies atômicas, que resulta na elevada polarizabilidade magnética do Pd. De um modo mais geral, esses resultados justificam entender a bicamada de $\mathrm{Pd} / \mathrm{Fe}$ como uma única entidade magnética. As direções dos momentos de spin para as camadas de Ir surtem pouco efeito para a descrição do sistema, haja vista sua pequena magnitude em relação aos momentos calculados para as camadas de Pd e Fe. 
Tabela 5.3: Ângulos $\theta_{n}$ calculados (em graus) entre o $n$-ésimo átomo da vizinhança com o átomo central, para as camadas de $\mathrm{Pd}$ e de $\mathrm{Fe}$ no sistema $\mathrm{Pd} / \mathrm{Fe} / \mathrm{Ir}(111)$. Para esse cálculo, foi considerado o aglomerado de átomos não equivalentes apresentado na Fig. $5.2(\mathrm{~b})$.

\begin{tabular}{cccccccc}
\hline Sistema & Vizinhança & $\theta_{1}$ & $\theta_{2}$ & $\theta_{3}$ & $\theta_{4}$ & $\theta_{5}$ & $\theta_{6}$ \\
\hline \hline \multirow{3}{*}{ Fe } & $1^{a}$ & $79^{\circ}$ & $63^{\circ}$ & $78^{\circ}$ & $63^{\circ}$ & $66^{\circ}$ & $62^{\circ}$ \\
& $2^{a}$ & $140^{\circ}$ & $156^{\circ}$ & $132^{\circ}$ & $133^{\circ}$ & $127^{\circ}$ & $128^{\circ}$ \\
& $3^{a}$ & $178^{\circ}$ & $153^{\circ}$ & $170^{\circ}$ & $163^{\circ}$ & $145^{\circ}$ & $152^{\circ}$ \\
\hline \hline \multirow{3}{*}{$\mathbf{P d}$} & $1^{a}$ & $86^{\circ}$ & $89^{\circ}$ & $78^{\circ}$ & $74^{\circ}$ & $70^{\circ}$ & $77^{\circ}$ \\
& $2^{a}$ & $145^{\circ}$ & $135^{\circ}$ & $116^{\circ}$ & $103^{\circ}$ & $121^{\circ}$ & $146^{\circ}$ \\
& $3^{a}$ & $172^{\circ}$ & $142^{\circ}$ & $117^{\circ}$ & $116^{\circ}$ & $116^{\circ}$ & $151^{\circ}$ \\
\hline
\end{tabular}

É conhecido na literatura [33] que, para pequenos clusters depositados em superfícies magnéticas, diferentes configurações não-colineares quase degeneradas podem ocorrer para o mesmo sistema, de maneira que o estado fundamental é caracterizado por flutuações entre essas configurações. Embora a multicamada $\mathrm{Pd} / \mathrm{Fe} / \mathrm{Ir}(111)$ investigada neste trabalho seja composta de um substrato não-magnético, verificamos a eventual existência (ou inexistência) de mínimos com energia muito próxima tomando diversos arranjos iniciais de momentos de spin para os cálculos não-colineares do aglomerado representado na Fig. 5.2(b), entre eles considerando, por exemplo: todos os momentos alinhados com o eixo- $z$; todos os momentos alinhados com o eixo perpendicular ao plano de superfície; e configurações iniciais aleatórias de spin. Como resultado obtivemos que, em todos os casos investigados, o estado não-colinear final apresentou diferenças desprezíveis, tanto em energia (da ordem de $10^{-1} \mathrm{meV} /$ átomo, certamente dentro do erro estimado para a aproximação ASA [186]) como na estrutura de spins, demonstrando ser essencialmente independente da escolha do arranjo inicial.

Embora seja visível a existência de uma configuração não-colinear de spins no estado fundamental da multicamada $\mathrm{Pd} / \mathrm{Fe} / \mathrm{Ir}(111)$, sua origem microscópica ainda não foi estudada em detalhes. Até agora, os resultados obtidos via cálculos de primeiros princípios mostraram que dois efeitos simultâneos podem ser responsáveis pela emergência de texturas complexas de spin no estado fundamental desse material, a saber: $(i)$ a significativa frustração magnética de origem nas interações de troca; e $(i i)$ o forte acoplamento de DM verificado para os pares de átomos Fe-Fe. De um modo mais geral, R. Lizárraga et al. [58] argumentam que o magnetismo não-colinear pode surgir em um material qualquer quando há um equilíbrio favorável entre o preenchimento e o exchange splitting das bandas majoritária e minoritária de spin. Assim, com a finalidade de isolar um desses efeitos, e lembrando que Moriya [49] descreve a interação anisotrópica entre spins como uma consequência do acoplamento spin-órbita, realizamos cálculos não-colineares sem considerar 
o efeito desse acoplamento na autoconsistência. Conforme explicado na referência [187], o fato de não considerarmos o termo de acoplamento spin-órbita acarreta na inexistência de um eixo preferencial de magnetização no sistema, e a convergência ocorre para cada momento magnético de spin em relação a todos os outros no aglomerado. Portanto, caso todos os spins estejam voltados para uma mesma direção, essa configuração permanecerá até que se atinja a convergência. Da mesma forma que nos cálculos não-colineares anteriores, diversos arranjos iniciais de spin foram analisados - a fim de minimizar o risco de não encontrarmos uma configuração magnética correspondente ao mínimo de energia global. Entre esses arranjos, consideramos as seguintes situações: ( $i$ ) todos os momentos de spin voltados para a direção perpendicular à superfície de $\operatorname{Ir}(111)$ ([111]); (ii) todos os momentos paralelos ao vetor [111], exceto o spin do átomo de Fe central, que foi deslocado para uma direção aleatória; e (iii) cada momento de spin voltado à direções aleatórias e diferentes entre si.

Para o caso $(i)$, no qual todos os momentos de spin estão inicialmente arranjados em uma configuração colinear, o resultado final (convergido) preservou a colinearidade do sistema. Por outro lado, tanto ao se alterar a direção apenas do momento de spin do Fe central (caso (ii)), quanto ao se iniciar o cálculo com um arranjo aleatório de spins (caso (iii)), a autoconsistência resultou em soluções não-colineares como estado fundamental. A diferença de energia entre as soluções não-colineares e colinear encontradas é relativamente pequena, da ordem de $\sim 5 \mathrm{meV} /$ átomo em favor do estado colinear. Em outras palavras, essa análise nos indica que a frustração magnética devido à competição FM-AFM entre as interações de troca não é suficiente, sozinha, para conduzir o sistema ao estado nãocolinear apresentado na Fig. 5.7, mas é significativamente grande para que as soluções não-colineares representem, neste cenário, mínimos locais metaestáveis. Nesse sentido, em sistemas similares, tais como a bicamada de $\mathrm{Pt}_{1-x} \mathrm{Ir}_{x} /$ Fe depositada em $\operatorname{Pd}(111)$, L. Rózsa et al. demonstraram recentemente [56] que as interações isotrópicas do tipo de Heisenberg, de fato, participam ativamente na criação de um estado fundamental caracterizado pela configuração em espiral de spin, e que a frustração magnética leva a forças atrativas entre skyrmions quando estes estão separados por pequenas distâncias.

Dessa forma, pode-se dizer que ambos os efeitos das interações de troca e de DM são importantes para a emergência do magnetismo não-colinear no sistema $\mathrm{Pd} / \mathrm{Fe} / \mathrm{Ir}(111)$.

Antes de prosseguirmos, porém, convém comentar um pouco sobre a anisotropia magnética dessa bicamada. Por se tratar essencialmente de um sistema bidimensional, assumimos aqui a existência de uma anisotropia uniaxial na qual, devido à simetria reduzida em uma das direções, o eixo de fácil magnetização deve direcionar-se paralela ou 
perpendicularmente ao plano de superfície. Dado que $\Delta m_{L}$ é definido como uma quantidade local, é numericamente viável obtê-la a partir de cálculos de primeiros princípios (tais como o RS-LMTO-ASA [120]), apresentando ainda a vantagem de se constituir um valor bem definido para o método adotado neste trabalho. A partir da Eq. 2.21, é direto verificar que o eixo de fácil magnetização do material será paralelo à direção de máximo momento orbital. Por outro lado, como é possível perceber pela Fig. 5.1(b), ao se calcular a energia de anisotropia da camada de Fe, nota-se que a banda majoritária de spins se encontra quase totalmente preenchida, de maneira que a maior contribuição à formação do momento orbital no Fe vem da banda minoritária de spins $(\sim 97 \%)$.

Com base em todas as considerações anteriores, e sobretudo na Eq. 2.21, calculamos as projeções do momento orbital de um átomo de Fe típico nos eixos globais de magnetização [111] (perpendicular à superfície) e [11̄0] (paralelo à superfície). Como consequência, obtivemos que $E_{\mathrm{MAE}}=\frac{-\xi_{\mathrm{Fe}}}{4 \mu_{B}}\left(m_{L_{[111]}}-m_{L_{[110]}}\right) \sim 0,1 \mathrm{meV}$ por átomo de $\mathrm{Fe}$ em favor do estado cuja magnetização é perpendicular ao plano de superfície, concordando com o reportado pela literatura [54, 60, 98] Apenas para registro, a constante de acoplamento spin-órbita do Fe, calculada na condição de uma monocamada no sistema $\mathrm{Pd} / \mathrm{Fe} / \mathrm{Ir}(111)$, é de $\xi_{\mathrm{Fe}}=107,7 \mathrm{meV}$, apenas ligeiramente menor quando comparada à constante obtida para o Fe bulk (bcc), de $\xi_{\mathrm{Fe}}=108,5 \mathrm{meV}$, utilizando o mesmo parâmetro de rede experimental de $2,87 \AA[188]$.

Com os parâmetros ${ }^{1}$ obtidos via cálculos de primeiros princípios, realizamos posteriormente simulações da dinâmica atomística de spins baseadas na equação de movimento de LLG (veja seção 4). Para esse fim, utilizamos a versão mais recente do código Uppsala Atomistic Spin Dynamics (UppASD) [164, 165]. Inicialmente, estabilizamos a configuração magnética à temperaturas finitas via método de Monte Carlo (MC) (seção 4.3), para em seguida realizarmos os cálculos de dinâmica. Em termos práticos, as excitações no sistema são geradas por rotações clássicas nos spins atômicos individuais, de tal forma que sua energia satisfaça a estatística de Boltzmann (via algoritmo de Metropolis-Hastings). No estágio de aplicação do método $\mathrm{MC}$, que chamaremos daqui em diante de fase inicial do sistema, a estabilização à uma temperatura final desejada $T_{\text {final }}>0$ ocorreu, em todos os casos investigados, em 6 (seis) etapas de temperaturas decrescentes igualmente espaçadas em porcentagem, respeitando o seguinte algoritmo. Em um primeiro momento, um teste foi realizado para se estimar a temperatura de Curie do sistema $\left(T_{C}^{\text {est }}\right)$, a qual para a monocamada de Fe no sistema $\mathrm{Pd} / \mathrm{Fe} / \mathrm{Ir}(111)$ foi estabelecida, por teoria de campo médio, em $T_{C}^{\text {est }}=950,8 \mathrm{~K}$. Assim, se $T_{\text {final }} \leq 70 \mathrm{~K}$, então a primeira temperatura de estabilização $\left(T_{1}\right)$ é tal que $T_{1}=200 \mathrm{~K}$ ou $T_{1}=0,9 \cdot T_{C}^{\text {est }}$, o que fosse menor. No caso

\footnotetext{
${ }^{1}$ Momentos magnéticos locais (de spin), interações de troca e de DM.
} 
de $70<T_{\text {final }} \leq 120 \mathrm{~K}$, adotou-se $T_{1}=300 \mathrm{~K}$ ou $T_{1}=0,9 \cdot T_{C}^{\text {est }}$, considerando o menor valor dentre as duas possibilidades. Por fim, para $T_{\text {final }}>120 \mathrm{~K}$, então $T_{1}=2,5 \cdot T_{\text {final }}$ ou $T_{1}=0,9 \cdot T_{C}^{\text {est }}$, o que fosse menor. Estabelecido o valor de $T_{1}$, as temperaturas subsequentes envolvidas na fase inicial foram obtidas pela relação $T_{n}=T_{1}\left(\frac{T_{\text {final }}}{T_{1}}\right)^{\frac{(n-1)}{5}}$, de forma que $T_{6} \equiv T_{\text {final }}$. Esse procedimento foi realizado até a temperatura final máxima de $T_{\text {final }}=400 \mathrm{~K}$. É relevante destacar que a descrição da fase inicial é de suma importância para a obtenção de uma boa simulação dinâmica da magnetização. Dependendo da fase inicial, para determinados valores de campo magnético externo a emergência de skyrmions é metaestável e pode coexistir com o estado de espiral de spin no material [54].

Sobre os cálculos de dinâmica de spins para esse sistema, é importante ressaltar que a simulação apenas da monocamada magnética (Fe) é suficiente para reproduzir os resultados observados experimentalmente [52]. De fato, sugerimos que a influência das demais camadas de $\mathrm{Pd}$ e Ir já está incorporada nos parâmetros $\mathcal{J}_{i j}$ e $\mathbf{D}_{i j}$ obtidos via primeiros princípios, haja vista que a estrutura eletrônica local do Fe foi convergida considerando-se as camadas metálicas adjacentes. Logo, calculada a fase inicial do sistema para diferentes temperaturas $\left(0<T_{\text {final }} \leq 400 \mathrm{~K}\right)$, realizamos a dinâmica de spins descrita pela equação de movimento de LLG (Eq. 4.1) [63, 164]. Em todas as simulações, escolhemos um tempo total de $13 \mathrm{ps}$, sempre considerando intervalos de tempo de $\Delta t=10^{-16} \mathrm{~s}$ para a solução numérica da equação diferencial. Após diversos testes, notamos que, para uma descrição magnética adequada do sistema, é necessário que a Hamiltoniana de spin inclua as interações de troca e de DM de mais longa distância. Por exemplo, ao se fazer um teste com os parâmetros até a terceira camada de vizinhos (isto é, com uma distância máxima de $\sim 5,43 \AA$ ), encontra-se uma espiral de spin como estado fundamental, mas (a menos de uma situação instável na qual o campo externo aplicado é próximo de 12 T), não se obtém uma fase caracterizada por uma rede de skyrmions - apenas anti-skyrmions isolados e que não condizem com a observação experimental. Já ao se diminuir o raio de interações, considerando os acoplamentos até a segunda camada de vizinhos ( $\mathrm{a} \sim 4,70 \AA$ do átomo de Fe central, típico da superfície), encontramos um estado fundamental FM, como era de se esperar. Isso porque, apesar de considerarmos a interação de DM entre pares de primeiros vizinhos Fe-Fe (que, por estar direcionada quase no plano da superfície de $\operatorname{Ir}(111)$ induz uma rotação dos momentos de spin para fora do plano [54]), o acoplamento AFM com a terceira camada de vizinhos Fe-Fe não é incluído e, portanto, trata-se de um sistema no qual não há frustração magnética. Ademais, os cálculos de dinâmica confirmam o que já havia sido demonstrado por primeiros princípios (Fig. 5.7): o magnetismo nãocolinear observado no $\mathrm{Pd}[52,59]$ é induzido pela camada de Fe. Ao realizarmos um teste considerando apenas a camada de Pd com suas respectivas interações, o estado fundamen- 
tal, independentemente da ação de um campo magnético externo, resultou em todos os cálculos incluindo acoplamento spin-órbita em uma configuração FM (já que $\mathcal{J}_{\mathrm{Pd}-\mathrm{Pd}}>0$ para pares de primeiros vizinhos). Assim, considerando todo o raio de interações calculadas (Figs. 5.3 e 5.4), e para $T_{\text {final }}=10^{-4} \mathrm{~K}\left(T_{\text {final }} \sim 0 \mathrm{~K}\right)$ e campo magnético externo nulo, o resultado foi compatível com o observado experimentalmente [52]: um estado fundamental caracterizado por uma espiral de spin (carga topológica total $Q=0$ ). A Figura 5.8, abaixo, mostra uma representação esquemática da configuração obtida para uma matriz hexagonal contendo $100 \times 100$ sítios atômicos, cada um associado à um único spin. O comprimento de onda médio calculado para que os spins retornem à sua direção original é de $\bar{\lambda} \sim 10,7 \mathrm{~nm}$ - um pouco maior que o observado experimentalmente, de $6-7$ $\mathrm{nm}[52]$.

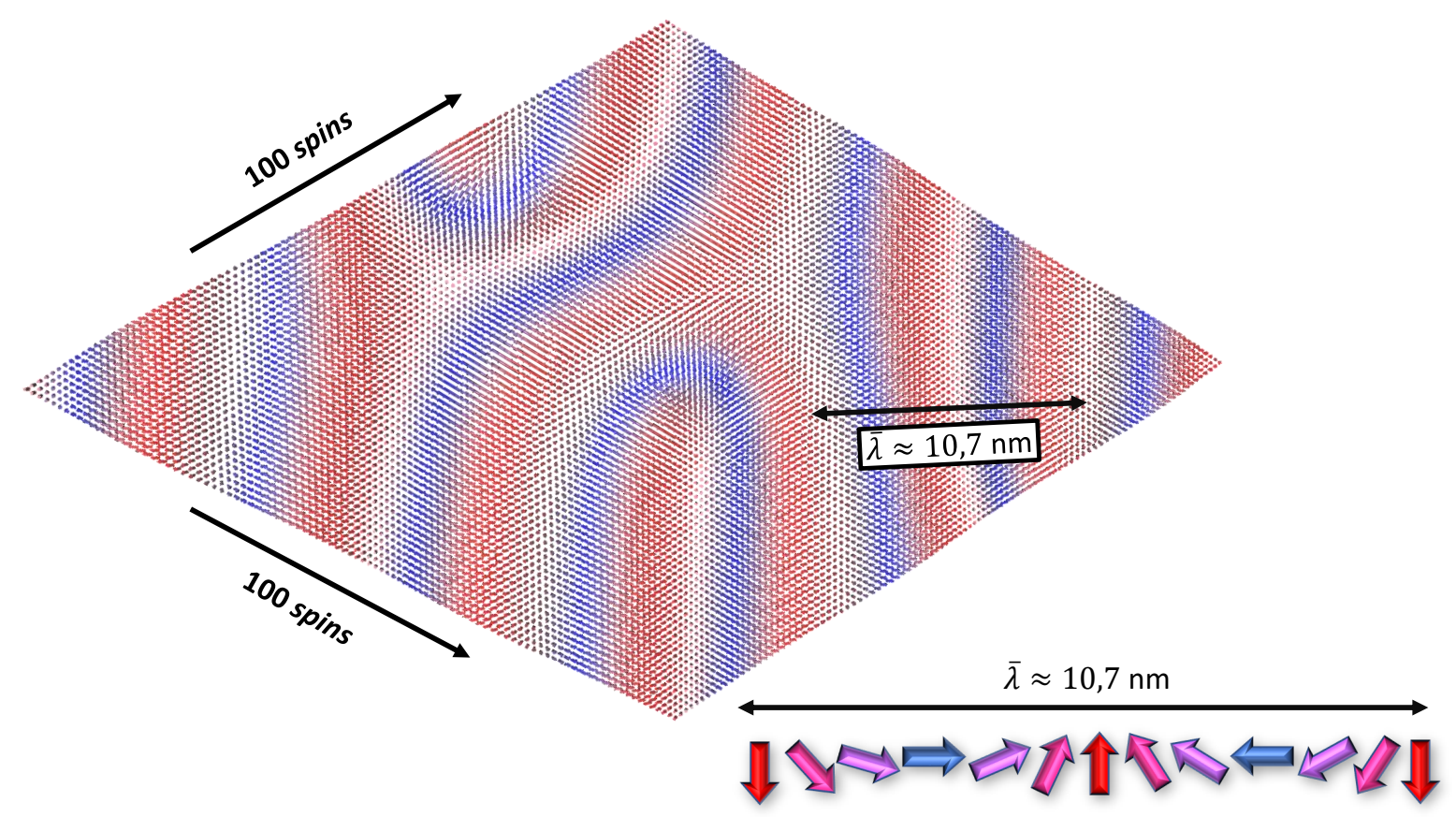

Figura 5.8: Resultado para a simulação de dinâmica atomística de spins no sistema $\mathrm{Pd} / \mathrm{Fe} / \mathrm{Ir}(111$ ) (aqui mostrada apenas a textura magnética da camada de $\mathrm{Fe}$ ) a $T \sim 0 \mathrm{~K}$ e campo magnético nulo, considerando-se uma matriz de $100 \times 100$ spins.

Por outro lado, para um campo $\mathbf{B}$ de magnitude $~ 1,6 \mathrm{~T}$, aplicado perpendicularmente à superfície de $\operatorname{Ir}(111)$, observamos a emergência de uma rede de skyrmions, como pode ser visto na Figura 5.9(a). Sob essas circunstâncias, favorece-se o surgimento de skyrmions com carga topológica $q=-1$ (calculado), nos quais o sentido único de rotação conduz gradualmente as direções dos momentos de spin para fora do plano (Fig. 5.9(a) inset), classificando-se como skyrmions do tipo Néel. Esses resultados estão de acordo com o reportado pela referência [53]. Nota-se, também que, devido às interações ligeiramente distintas (e com diferentes origens, como veremos mais adiante), os skyrmions apresentam 
uma certa assimetria, com uma maior extensão em um dos eixos. De fato, seu formato no plano é elíptico e o eixo maior possui um comprimento médio de $\sim 13,2 \mathrm{~nm}$, enquanto que o eixo menor apresenta um comprimento médio de $\sim 9,6 \mathrm{~nm}$. Essa rede de skyrmions é, então, progressivamente rarefeita com o aumento da intensidade do campo magnético, até que se atinja um valor crítico $B_{c}$, a partir do qual o sistema se torna monodomínio (com os spins paralelos a B). A partir das simulações realizadas neste trabalho, calculamos $B_{c} \sim 3$ T. Por sua vez, a Figura 5.9(b), para $B \sim 2,6 \mathrm{~T}$, retrata uma densidade menor de skyrmions na matriz de spins, devido ao aumento do campo magnético em relação à Fig. 5.9(a). 

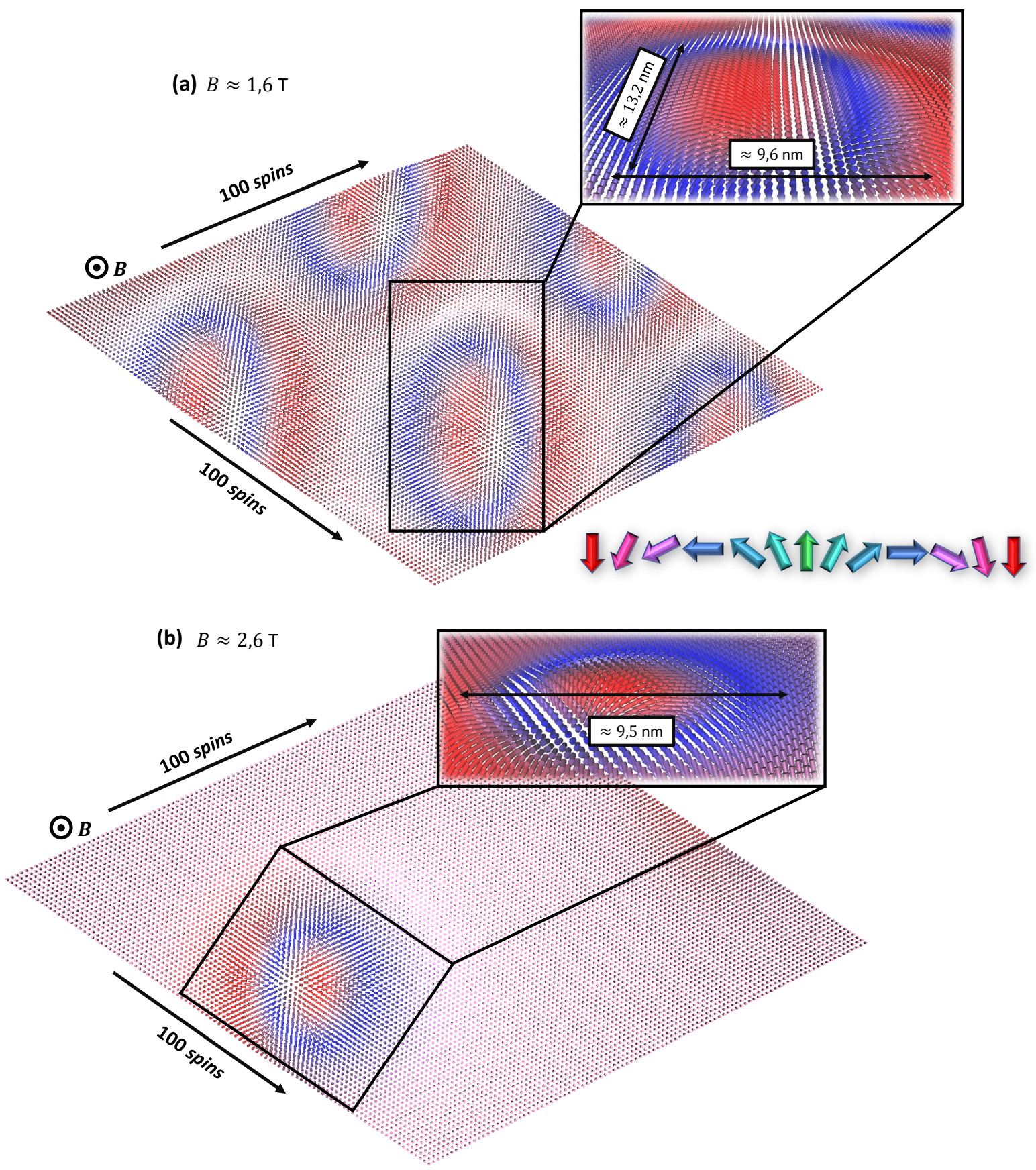

Figura 5.9: Resultado para a simulação de dinâmica atomística de spins no sistema $\mathrm{Pd} / \mathrm{Fe} / \mathrm{Ir}(111)$ (aqui mostrada apenas a textura magnética da camada de $\mathrm{Fe}$ ) a $T \sim 0 \mathrm{~K}$ e campos magnéticos $(a) B \sim 1,6 \mathrm{~T}$; e $(b) B \sim 2,6 \mathrm{~T}$ (ambos perpendiculares à superfície), considerando-se uma matriz de $100 \times 100$ spins.

É interessante verificar que, conforme o resultado apresentado na Fig. $\mathbf{5 . 9 ( b ) , ~ o ~}$ tamanho e o formato dos skyrmions são fortemente dependentes do campo magnético externo aplicado, um resultado análogo ao reportado pela referência [55]. Nesse caso específico, o aumento de $\sim 1 \mathrm{~T}$ na magnitude de $\mathbf{B}$ modificou o formato elíptico dos 
skyrmions para uma configuração essencialmente circular, com diâmetro de $\sim 9,5 \mathrm{~nm}$ (isto é, manteve-se o comprimento do eixo menor e reduziu-se em $\sim 28 \%$ o comprimento do eixo maior dos skyrmions sujeitos a $B \sim 1,6 \mathrm{~T}$ ), tal como mostra o inset da Fig. $5.9(\mathrm{~b})$.

A obtenção de uma rede de skyrmions nas simulações utilizando o pacote UppASD, quando o sistema é sujeito à um campo magnético externo de magnitude semelhante à observada experimentalmente [52], motivou a elaboração de um diagrama de fases (magnéticas) para a bicamada de $\mathrm{Pd} / \mathrm{Fe} / \mathrm{Ir}(111)$. Como mencionado anteriormente (seção 2.6.1), a carga topológica $q$ configura-se como uma boa quantidade para se caracterizar a presença de skyrmions em um determinado arranjo não-colinear de spins, além de sua quiralidade. Portanto, uma forma de construir o diagrama de fases é calcular $q$ para cada par $(B, T)$ de campo magnético externo aplicado e temperatura, visando obter as regiões nas quais $q \neq 0$. Por outro lado, é importante lembrar que, a partir de determinada temperatura, os skyrmions tornam-se instáveis, isto é, com duração finita, sobretudo pelo efeito das flutuações térmicas. Assim, é natural que a carga topológica seja calculada dinamicamente, ou seja, como uma quantidade dependente do tempo $(q=q(t))$, e o diagrama de fases seja construído com base na carga média $\bar{q}=\frac{\sum_{t=0}^{t_{t o t a l}} q(t)}{t_{\text {total }}} 1$ para cada par $(B, T)$. Ademais, cabe ressaltar que, como bem exemplifica a Fig. 5.9(a), nem sempre haverá apenas 1 (um) skyrmion na matriz de spins considerada, e, ao se calcular a carga topológica total do sistema, o resultado será proporcional ao número de skyrmions presentes naquela configuração magnética. Em outras palavras, podemos definir uma carga topológica total $Q=n q$ para cada arranjo de spins sujeito à um campo magnético $B$ e temperatura $T$, onde $n$ é número de skyrmions existentes no sistema. Portanto, a melhor quantidade para se caracterizar um dado arranjo de spins é a carga média $\bar{Q}=n \bar{q}^{2}$. Nesse sentido, um diagrama de fases sugerido para o sistema $\mathrm{Pd} / \mathrm{Fe} / \operatorname{Ir}(111)$ é apresentado na Figura 5.10, na qual se verifica a clara predominância de skyrmions com carga topológica $q=-1$ (já que $\bar{Q}$ assume, em geral, um valor negativo nas regiões em que é não-nulo).

\footnotetext{
${ }^{1}$ Aqui, $t_{\text {total }}$ representa o tempo total da dinâmica de spins (13 ps), e cada termo do somatório é a carga topológica $q$ calculada no tempo $t$ atual, que varia com intervalos definidos pelo passo da simulação $\left(\Delta t=10^{-16} \mathrm{~s}\right)$.

${ }^{2}$ De fato, essa definição é válida apenas para baixas temperaturas, nas quais o número de skyrmions no sistema não varia com o tempo. Para altas temperaturas (sobretudo na fase paramagnética), os skyrmions são extremamente instáveis e o número $n$ é dependente do tempo e entra no somatório para se calcular a carga média como uma função $n(t): \bar{Q}=\frac{\sum_{t=0}^{t_{\text {total }}} n(t) q(t)}{t_{\text {total }}}$.
} 


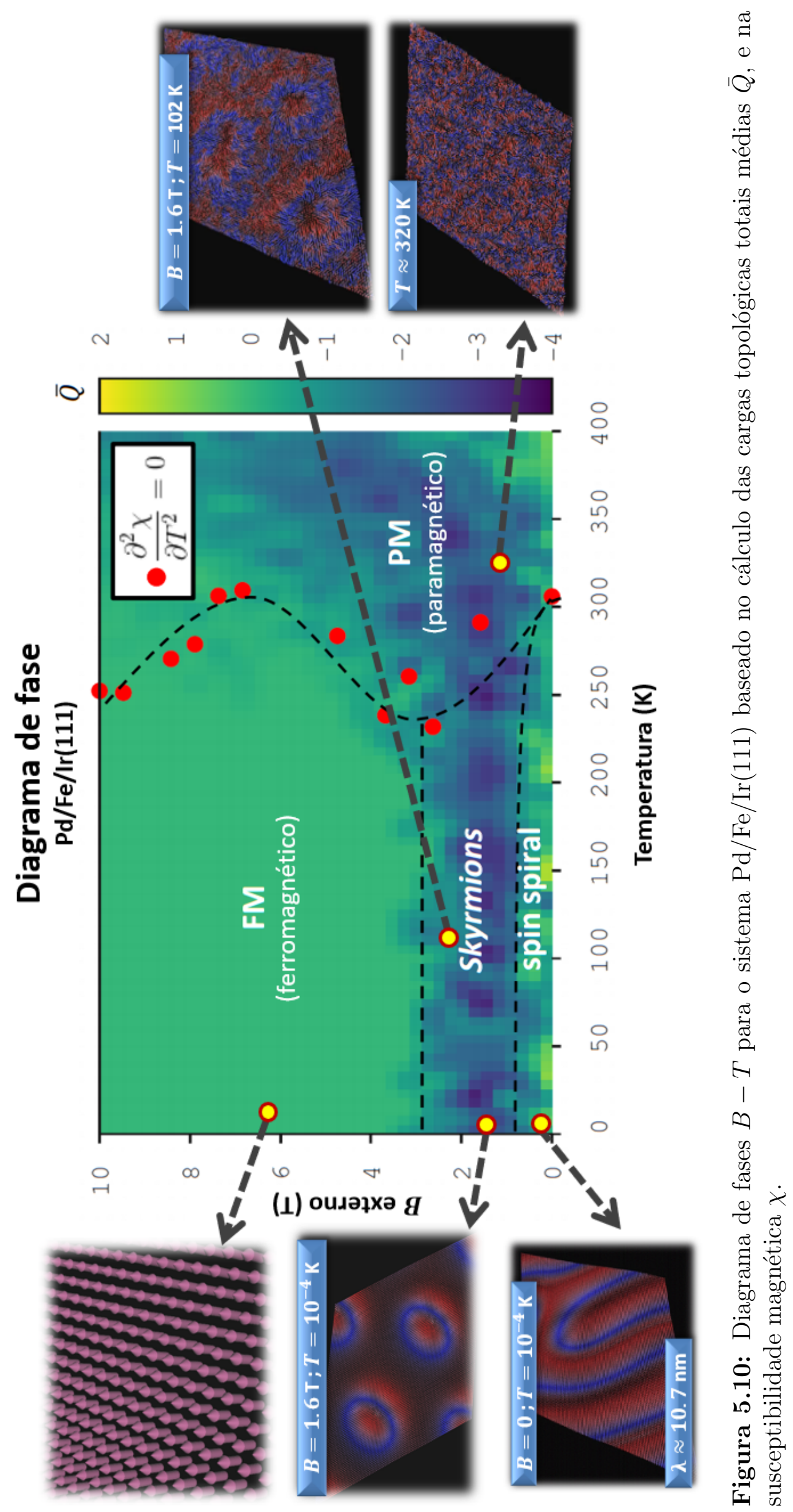


Os valores marcados em vermelho no gráfico da Fig. 5.10 são os pontos de inflexão das curvas de susceptibilidade magnética como função da temperatura $\chi(T)$, ou seja, o resultado de $\frac{\partial^{2} \chi(T)}{\partial T^{2}}=0$ para cada campo externo $B$ fixo. É sabido da física estatística que as transições de fase ocorrem quando existe uma singularidade na energia livre ou em suas derivadas, caracterizando um comportamento não-analítico das grandezas físicas associadas. Dentre as quantidades que podem sofrer uma ruptura no comportamento analítico devido à transição de fase está a susceptibilidade magnética. Essa, por sua vez, pode ser definida em termos da magnetização média por sítio $\langle m\rangle=\frac{1}{N} \sum_{i=1}^{N} m_{i}$, e da magnetização quadrática média $\left\langle m^{2}\right\rangle=\frac{1}{N} \sum_{i=1}^{N} m_{i}^{2}$ do sistema por

$$
\chi=\frac{1}{k_{B} T}\left(\left\langle m^{2}\right\rangle-\langle m\rangle^{2}\right) .
$$

Isto posto, os pontos de inflexão da função $\chi(T)$ para cada campo $B$ aplicado devem indicar a região na qual existe uma transição de fase magnética de segunda ordem no sistema. Essas funções foram obtidas tomando-se os valores de $\chi$ dependentes da temperatura para cada campo magnético (40 pontos no total), sendo posteriormente ajustada uma função quártica $\left(a x^{4}+b x^{3}+c x^{2}+d x+e\right)$ a cada conjunto de pontos.

Verifica-se, pelo diagrama da Fig. 5.10, que a bicamada de $\mathrm{Pd} / \mathrm{Fe} / \mathrm{Ir}(111)$ apresenta uma região de valores $(B, T)$ na qual a densidade de skyrmions é elevada, particularmente para campos magnéticos aplicados com magnitude de $1 \mathrm{~T} \lesssim B \lesssim 3 \mathrm{~T}$. Nessa região, tal como mostra a Fig. 5.9, a camada de Fe é caracterizada por uma rede de skyrmions, com estrutura aproximadamente regular e cada vez mais esparsa conforme se aumenta a intensidade do campo (veja também a referência [98]). Já para campos $B \gtrsim 3 \mathrm{~T}$, o material torna-se um monodomínio FM até temperaturas $T \sim 250 \mathrm{~K}$, sobretudo devido à saturação provocada pelo elevado valor de $B$ e relacionado à significativa importância do termo Zeeman na Hamiltoniana de spin sob essas condições. Uma região caracterizada majoritariamente por espirais de spin também foi identificada para $B \lesssim 1 \mathrm{~T}$, sendo que as soluções encontradas apresentam uma estrutura magnética semelhante à obtida para o estado fundamental do sistema (Fig. 5.8). Esses resultados estão de acordo com o reportado por L. Rózsa et al. [53].

As transições de fase de segunda ordem discutidas anteriormente e avaliadas de maneira quantitativa por meio da identidade $\frac{\partial^{2} \chi(T)}{\partial T^{2}}=0$ para cada campo $B$ constituem uma fronteira no diagrama a partir da qual calculou-se uma carga topológica média $\bar{Q} \neq 0$ em quase todo o intervalo de campos aplicados, até $B=10$ T. Nessa região, as estruturas magnéticas tipo-skyrmion são metaestáveis e, em muitos casos, apresentam cargas 
topológicas fracionárias - o que, portanto, condiz com a existência de uma fase paramagnética (PM), dominada pelos efeitos de flutuações térmicas. Entretanto, é interessante notar que, para temperaturas ligeiramente menores às calculadas para a transição à fase PM, encontramos skyrmions não-permanentes, isto é, com curto tempo de vida. Sendo os skyrmions entidades físicas com propriedades topológicas, a existência de estruturas temporárias pode ser facilmente verificada pela variação de $Q$ no tempo $(Q(t))$, como pode ser observado na Fig. 5.11 para um campo fixo $B=1,58 \mathrm{~T}$ (perpendicular à superfície de $\operatorname{Ir}(111))$. Ao contrário da fase PM, as estruturas magnéticas nessa região de transição apresentam apenas valores inteiros de $Q$ (Fig. 5.11(b)), embora estes não permaneçam constantes no tempo, como esperado. O curto tempo de vida, suficiente para ser registrado pelas simulações baseadas na dinâmica atomística de spins, permite que estabeleçamos uma estimativa fundamentada nas diferenças que a carga topológica total $Q$ do sistema assume ao longo do tempo. Para isso, é importante observar que, pela Fig. 5.11(b), o valor de $Q$ varia apenas uma unidade em módulo durante toda a simulação, o que fisicamente caracteriza a criação ou aniquilação de apenas um skyrmion na matriz de spins considerada. Mais especificamente, como a aplicação de um campo magnético perpendicular (out-of-plane) à superfície de $\operatorname{Ir}(111)$ tende a produzir skyrmions com carga topológica $q=-1$ na bicamada de $\mathrm{Pd} / \mathrm{Fe} / \mathrm{Ir}(111)$, o aumento de uma unidade no valor de $Q$ representa a aniquilação de um skyrmion no sistema, ao passo que a diminuição do valor de $Q$ em uma unidade representa a criação de mais uma dessas estruturas magnéticas.

A fim de calcular o tempo de vida, nós sugerimos compreender o gráfico da Fig. 5.11(b) como aquele que contém o ciclo de vida completo de apenas um skyrmion, tal como esquematizado na Fig. 5.12. Assim, é possível estabelecer o tempo de vida para um skyrmion $\left(\tau_{\mathrm{sk}}\right)$, à campo $B=1,58 \mathrm{~T}$ e temperatura $T=226 \mathrm{~K}$, como $\tau_{\text {sk }} \sim 7,9 \mathrm{ps}$. Esse tempo curto se deve, portanto, ao fato de analisarmos $\tau_{\text {sk }}$ próximo à temperatura de transição ao estado paramagnético. Devido à proposta de utilização dessas entidades físicas em novos dispositivos de gravação magnética, o estudo de sua estabilidade é algo fundamental e foi recentemente publicado na literatura [189], além de ter sido também investigado na Ref. [53]; entretanto, como não há uma regra geral, válida para todos os pares $(B, T)$ do diagrama de fases, não é possível fazer uma comparação direta com nossos resultados. Podemos inferir, contudo, que o tempo $\tau_{\text {sk }}$ reportado no presente trabalho certamente segue a tendência encontrada em [53]. Como comparação, é discutido nesses trabalhos que o tempo de vida de um skyrmion varia como uma função exponencial com a temperatura (antes, é claro, que se atinja a fase paramagnética), embora esse modelo tenha sido empregado apenas para a bicamada de $\mathrm{Pd} / \mathrm{Fe} / \mathrm{Ir}(111)$. Portanto, destacamos aqui uma lacuna no conhecimento do comportamento dinâmico dos skyrmions, e que se 
estabelece como um passo importante para uma real possibilidade de aplicação dessas estruturas magnéticas em novas tecnologias: entender quantitativamente o processo de criação/aniquilação de skyrmions para diferentes sistemas metálicos, devido ao efeito das flutuações térmicas. Por fim, cabe destacar que nossos cálculos levaram em conta um fator de damping de Gilbert de $\alpha=10^{-2}$ (adimensional). Essa informação é relevante, pois, como é sabido de trabalhos anteriores [167, 190], o damping afeta diretamente a velocidade com a qual o sistema pode operar, podendo influenciar no tempo de vida $\tau_{\text {sk }}$.

A variação temporal de $Q$ apresentada na Fig. 5.11(c), já no regime PM ( $T=308 \mathrm{~K})$, possibilita entender o porquê nessa região a carga topológica média $(\bar{Q})$ é diferente de zero, embora não se permita caracterizá-la como uma fase de skyrmions. Nota-se claramente que o valor de $Q$ flutua de maneira aleatória como função do tempo, de forma que, para intervalos suficientemente longos (i.e., muito maiores que os considerados nas presentes simulações), $\bar{Q}$ deve se aproximar de zero. Em outras palavras, a fase paramagnética sugerida no diagrama da Fig. $\mathbf{5 . 1 0}$ exibe valores não-nulos de $\bar{Q}$ (como seria esperado) pelo simples motivo de realizarmos simulações finitas, tanto no tempo quanto no tamanho da matriz de spins, sendo enviesada para valores de $\bar{Q}$ negativos. Essa mesma particularidade pode ser observada no diagrama de fases indicado na referência [53], embora os autores também classifiquem a região de altas temperaturas $(T \gtrsim 250 \mathrm{~K})$ como paramagnética. Outra característica observada nestas condições de campo magnético e temperatura que permitem a sua classificação como uma fase PM é o cálculo de valores de $\bar{Q}$ não-inteiros (por exemplo $\bar{Q}=-\frac{5}{3}$ ), o que fisicamente representaria nem a existência de skyrmions $(q= \pm 1)$, nem a existência de configurações magnéticas topologicamente triviais $(q=0)$. 

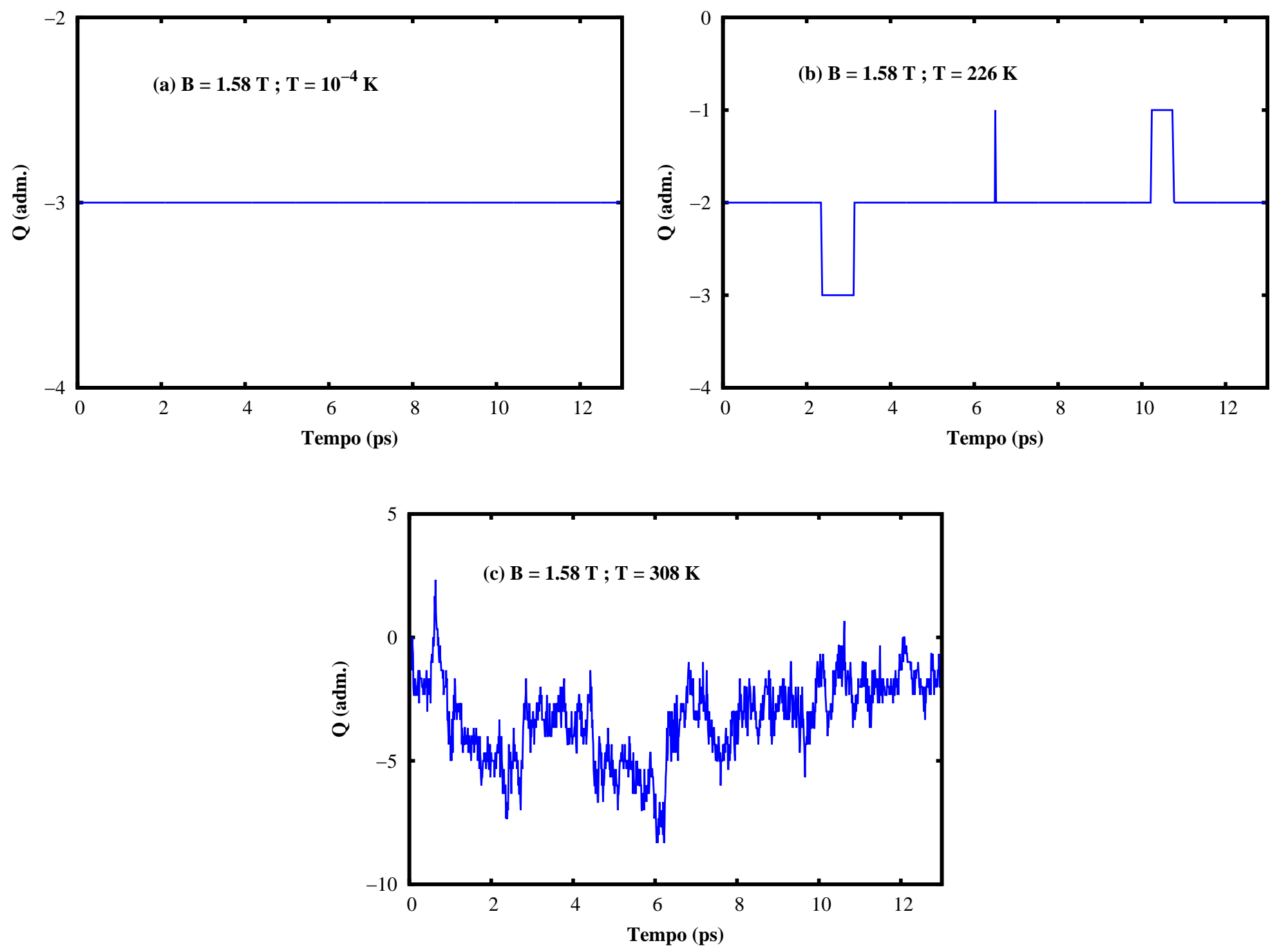

Figura 5.11: Carga topológica total $Q$ de um sistema de $100 \times 100$ spins com os parâmetros de interação da camada de Fe como função do tempo para diferentes temperaturas: (a) $10^{-4} \mathrm{~K}$; (b) $226 \mathrm{~K}$; e (c) $308 \mathrm{~K}$. 


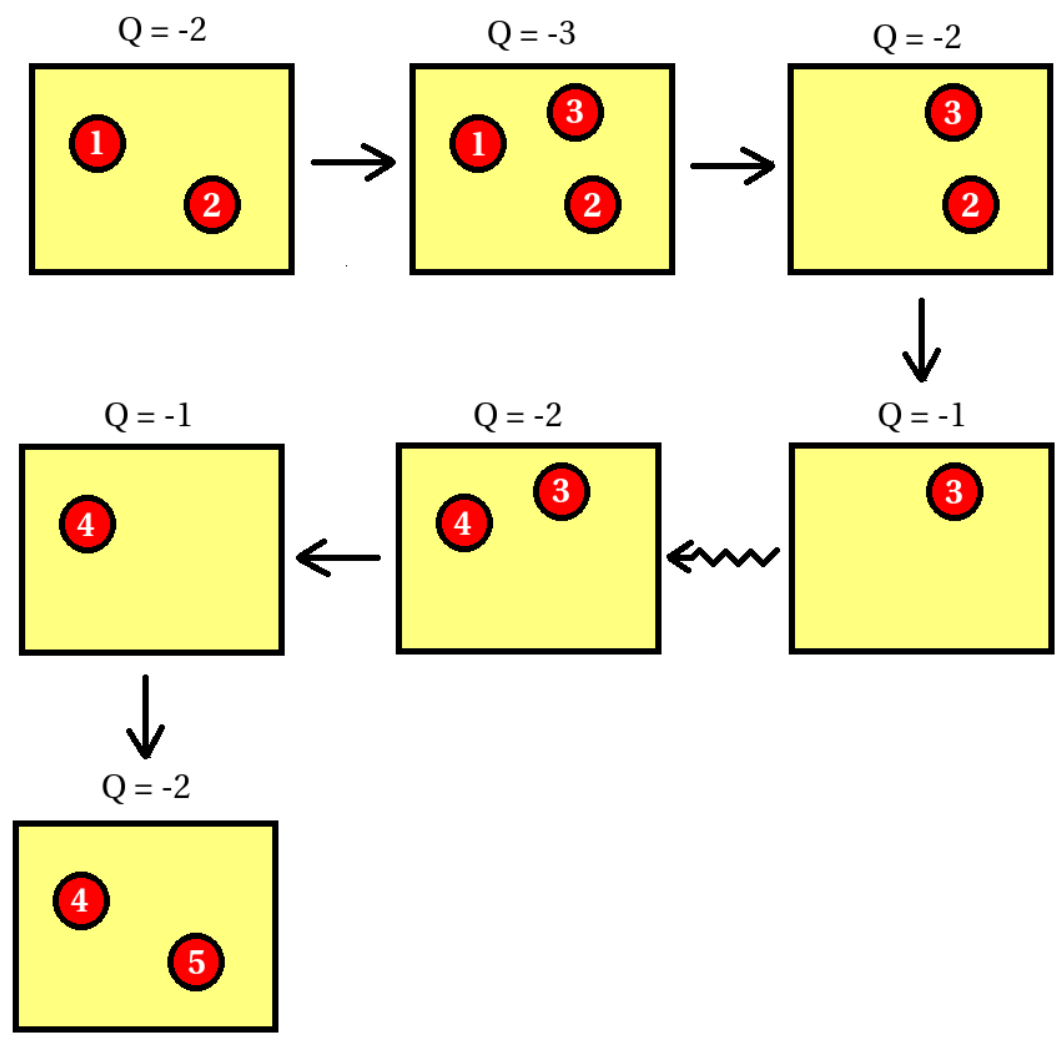

Figura 5.12: Esquema sugerido da evolução temporal do sistema de skyrmions cuja carga topológica total $Q$ é descrita pelo gráfico da Fig. 5.11(b). Cada retângulo em amarelo representa a matriz de spins e os círculos em vermelho, numerados, os skyrmions em ordem de criação/aniquilação. Por sua vez, a seta em zigue-zague representa a ocorrência de um rápido processo. Note que o único skyrmion cujo ciclo de vida foi completamente registrado é o identificado com o número "3".

\section{$5.2 \mathrm{Pd} / \mathrm{Co} / \operatorname{Ir}(111)$}

Como mencionado na seção 5.1, o propósito de se estudar a multicamada Pd/Co/Ir(111) advém da necessidade de se entender os efeitos físicos que são provocados pela mudança da camada magnética no sistema, de Fe para Co. Em outras palavras, pretende-se investigar as diferenças nas propriedades eletrônicas e magnéticas com o sistema $\mathrm{Pd} / \mathrm{Fe} / \mathrm{Ir}(111)$, quando um elétron de valência $3 d$ a mais é populado, em média, a cada orbital atômico $d$ na camada de $\mathrm{Fe}^{1}$. Até o presente momento, não há registros de investigações para a multicamada $\mathrm{Pd} / \mathrm{Co} / \mathrm{Ir}(111)$ na literatura, exceto o trabalho de L. V. Dzemiantsova et al. [61], que estabelece um estudo teórico e experimental das propriedades de átomos de Co sobre o sistema $\mathrm{Pd} / \mathrm{Co} / \mathrm{Ir}(111)$, tratando o $\mathrm{Pd}$ como um espaçador não-magnético. Ana-

\footnotetext{
${ }^{1}$ Essa interpretação está diretamente associada à distribuição eletrônica de valência de cada um desses elementos (Fe, Co) na condição de átomos isolados: $4 s^{2} 3 d^{6}$ para o $\mathrm{Fe}$, e $4 s^{2} 3 d^{7}$ para o Co.
} 
logamente à multicamada $\mathrm{Pd} / \mathrm{Fe} / \mathrm{Ir}(111)$, todos os resultados apresentados nesta seção - exceto para a análise do sistema a partir de cálculos não-colineares - foram realizados considerando todos os momentos de spin paralelos a uma única direção de magnetização (estado colinear), incluindo o termo de acoplamento spin-órbita na autoconsistência.

O cálculo da superfície de $\mathrm{Pd} / \mathrm{Co} / \mathrm{Ir}(111)$ teve como ponto de partida o mesmo sistema de Ir bulk previamente apresentado. Outrossim, as características de convergência da superfície foram análogas às descritas para o $\mathrm{Pd} / \mathrm{Fe} / \mathrm{Ir}(111)$, necessitando exatamente do mesmo número de camadas não-equivalentes de Ir, e também sendo simulada por um cluster no espaço real contendo $\sim 16.000$ átomos. Portanto, a Figura 5.13, a seguir, apresenta as LDOS de um átomo típico das camadas de $\mathrm{Pd}, \mathrm{Co}, \mathrm{Ir}_{1}$, e $\mathrm{Ir}_{5}$, bem como uma comparação entre as LDOS para os orbitais $d$ de átomos típicos das camadas de Co, Pd, e $\operatorname{Ir}_{1}$, e entre as LDOS totais obtidas para o $\operatorname{Ir}_{5}$ e para o Ir bulk. 

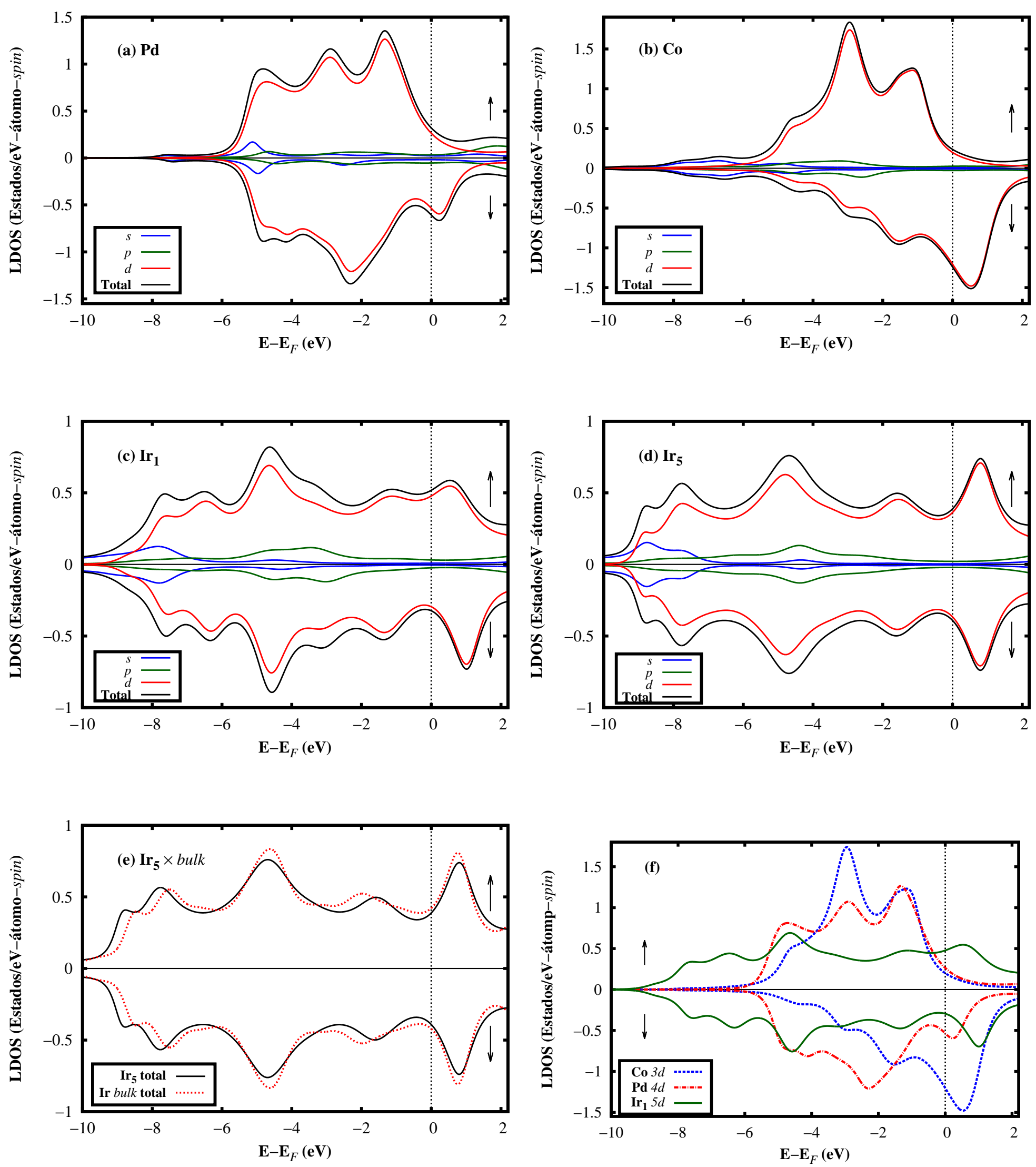

Figura 5.13: LDOS totais e projetadas nos obitais $s, p, d$ para a superfície de $\mathrm{Pd} / \mathrm{Co} / \mathrm{Ir}(111)$, considerando as seguintes situações: (a) camada de $\mathrm{Pd} ;(b)$ camada de Co; $(c)$ primeira camada de $\operatorname{Ir}\left(I r_{1}\right) ;(d)$ quinta camada de $\operatorname{Ir}\left(I r_{5}\right) ;(e)$ comparação entre as LDOS totais da última camada de Ir não-equivalente e de um átomo típico do Ir bulk $(f c c) ;(f)$ comparação entre as LDOS para os orbitais $d$ de átomos típicos das camadas de $\mathrm{Pd}, \mathrm{Co}, \mathrm{e} \mathrm{Ir}_{1}$. As setas $\uparrow \mathrm{e} \downarrow$ indicam as ocupações por spin nas bandas majoritária e minoritária, respectivamente. $E_{F}$ é a energia de Fermi do sistema $\left(=E_{F}\right.$ para o Ir bulk). 
É possível verificar que, novamente, a contribuição dominante para as LDOS advém dos estados $d$ de todos os elementos (Co, Pd, Ir) constituintes do sistema, como é de se esperar para metais de transição. A emergência de momentos magnéticos de spin não-nulos nas camadas de $\mathrm{Pd}$ e Ir estão relacionadas à existência de um exchange splitting entre as bandas majoritárias $(\uparrow)$ e minoritárias $(\downarrow)$ dos átomos típicos nessas camadas, claramente observado nas Figs. 5.13(a)-(c), resultando em ocupações eletrônicas locais que diferem da configuração dos sistemas bulk correspondentes. A convergência da densidade eletrônica da superfície de $\mathrm{Pd} / \mathrm{Co} / \mathrm{Ir}(111)$ é justificada pela Fig. 5.13(e), que evidencia apenas uma pequena diferença entre as LDOS da última camada não-equivalente incluída no cálculo $\left(\operatorname{Ir}_{5}\right)$ e do $\operatorname{Ir}$ bulk $(f c c)$. Finalmente, de maneira similar à bicamada de $\mathrm{Pd} / \mathrm{Fe} / \mathrm{Ir}(111)$, observa-se na Fig. 5.13(f) que há um significativo intervalo de energia no qual as LDOS dos Pd e do Co se sobrepõem, especialmente entre estados majoritários de spin, indicando uma elevada hibridização entre os orbitais $3 d-4 d$ de ambos. Ademais, novamente a comparação da Fig. 5.13(f) revela que os estados $5 d$ do Ir são mais estendidos em energia que os demais. Embora ainda se verifique uma elevada densidade de estados do Ir no nível de Fermi $\left(E=E_{F}\right)$, a pequena redistribuição de carga devido à associação com a camada de Co resulta em um valor $m_{S}^{\text {Ir }}$ reduzido para esse sistema. A Tabela 5.4, a seguir, lista os valores de $m_{S}$ e $m_{L}$ obtidos para cada átomo típico do sistema $\mathrm{Pd} / \mathrm{Co} / \mathrm{Ir}(111)$. Os resultados para as camadas de $\mathrm{Pd}, \mathrm{Co}$, e $\mathrm{Ir}_{1}$ apresentam boa concordância com os correspondentes reportados na referência [61].

Tabela 5.4: Momentos magnéticos de spin $\left(m_{S}\right)$ e orbitais $\left(m_{L}\right)$ calculados para um átomo típico de cada camada do sistema $\mathrm{Pd} / \mathrm{Co} / \mathrm{Ir}(111)$. Os valores são dados em $\mu_{B} /$ átomo.

\begin{tabular}{|c|c|c|}
\hline Camada & $\mathbf{m}_{\mathbf{S}}$ & $\mathbf{m}_{\mathbf{L}}$ \\
\hline $\mathbf{P d}$ & 0,28 & 0,03 \\
\hline $\mathbf{C o}$ & 1,80 & 0,10 \\
\hline $\mathbf{I r}_{1}$ & 0,07 & $-0,01$ \\
\hline $\mathbf{I r}_{2}$ & $-0,03$ & 0,02 \\
\hline $\mathbf{I r}_{3}$ & $<10^{-2}$ & $<10^{-2}$ \\
\hline $\mathbf{I r}_{4}$ & $<10^{-2}$ & $<10^{-2}$ \\
\hline $\mathbf{I r}_{5}$ & $<10^{-2}$ & $<10^{-2}$ \\
\hline
\end{tabular}

Nota-se que o momento de spin da primeira camada de $\operatorname{Ir}\left(\operatorname{Ir}_{1}\right)$, apesar de pequeno, alinha-se paralelamente à magnetização do Co. Conforme se aumenta a distância do Co, as camadas subjacentes de Ir apresentam um comportamento oscilatório quanto à direção de magnetização, ora alinhando-se paralelamente, ora ordenando-se de maneira antiparalela à $m_{S}^{\text {Co }}$. Esses momentos rapidamente decaem à zero com o aumento da distância à camada de Co, logo convergindo para a configuração eletrônica conhecida do Ir 
bulk $(f c c)$ - caracterizada pela ausência de exchange splitting entre as bandas majoritária e minoritária de spin.

Na Figura 5.14 são apresentados os valores médios das interações de troca $\left(\mathcal{J}_{i j}\right)$ entre pares de átomos Co-Co como função da distância de separação entre os constituintes de cada par. Nota-se que, diferentemente do calculado para a camada de Fe, os parâmetros de troca demonstram uma rápida redução para pequenos valores com o aumento da distância do par Co-Co, existindo valores negativos inexpressivos, tais como o observado para a interação com a quinta vizinhança (a $\sim 8,15 \AA$ de distância), de $\mathcal{J}_{\mathrm{Co}-\mathrm{Co}}=-0,39 \mathrm{meV}$ (ou $\sim 3 \%$ do valor médio de interação entre primeiros vizinhos, em termos absolutos). Nesse caso, a contribuição de troca efetiva é de $\mathcal{J}_{\mathrm{Co}-\mathrm{Co}}^{\text {eff }}=81,16 \mathrm{meV}$ para o acoplamento com a primeira vizinhança, e $\mathcal{J}_{\mathrm{Co}-\mathrm{Co}}^{\text {eff }}=-2,36 \mathrm{meV}$ para o acoplamento com a quinta vizinhança, o que corrobora com a conclusão anterior. Isso indica que não há tendência para frustração magnética entre os momentos de spins no sistema (pelo menos até a distância máxima de $\sim 9,41 \AA$ ). É também importante ressaltar que os primeiros vizinhos Co-Co são fortemente acoplados $\left(\mathcal{J}_{\mathrm{Co}-\mathrm{Co}}=13,53 \mathrm{meV}\right)$.

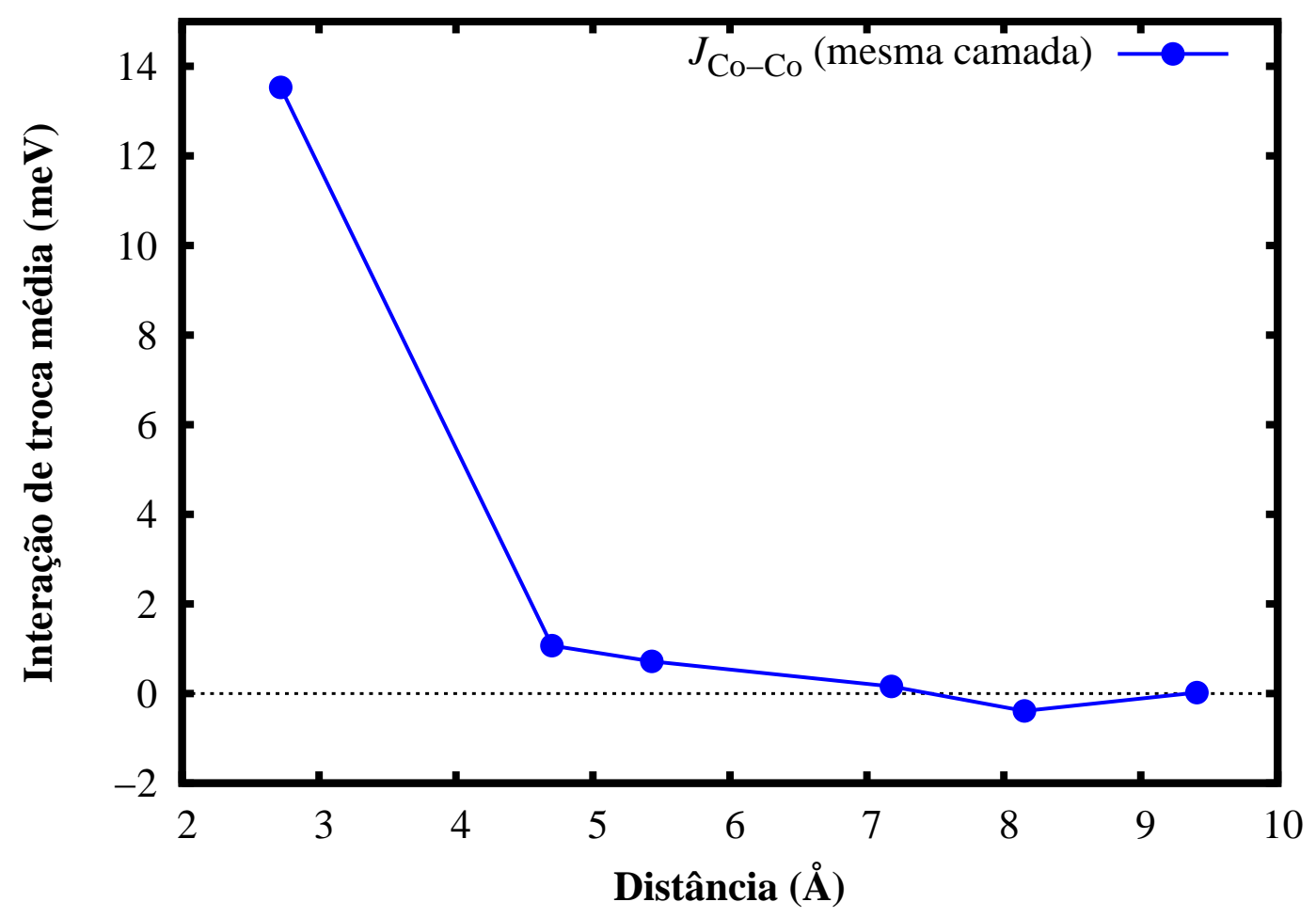

Figura 5.14: Valores médios das interações de troca entre pares de átomos Co-Co localizados na mesma camada $\left(\mathcal{J}_{\mathrm{Co}-\mathrm{Co}}\right)$, em função da distância entre os átomos do par.

Considerando as interações de troca entre camadas (Co-Pd, Co-Ir, e Pd-Ir), vale a pena 
mencionar a interação não-desprezível entre primeiros vizinhos de Co-Pd, com $\mathcal{J}_{\mathrm{Co}-\mathrm{Pd}}=$ 1,88 meV, sugerindo um acentuado acoplamento entre as camadas adjacentes de Co e Pd. Esse resultado está de acordo com o fato de haver uma significativa hibridização entre estados $4 d-3 d$ dos átomos de Co e Pd, respectivamente, demonstrado na Fig. 5.13(f). Isso, somado ao fato de que os momentos magnéticos induzidos no Pd são relativamente altos, permite que seja possível considerar que a bicamada de $\mathrm{Pd} /$ Co comporta-se como uma única entidade magnética. Embora o valor absoluto de $\mathcal{J}_{\mathrm{Co}-\mathrm{Pd}}$ seja menor que o encontrado para a interação de troca entre pares Fe-Pd $\left(\mathcal{J}_{\mathrm{Fe}-\mathrm{Pd}}\right)$, verifica-se um ligeiro aumento quando se consideram esses mesmos valores relativamente ao acoplamento de primeiros vizinhos $\mathrm{Co}-\mathrm{Co}$ ou $\mathrm{Fe}-\mathrm{Fe}$. O restante dos valores encontrados é pequeno, e não devem exercer significativa influência nas propriedades magnéticas da multicamada $\mathrm{Pd} / \mathrm{Co} / \operatorname{Ir}(111)$.

Além das interações de troca (isotrópicas), os acoplamentos anisotrópicos (de DM) no sistema $\mathrm{Pd} / \mathrm{Co} / \mathrm{Ir}(111)$ também sofrem algumas importantes alterações em relação à multicamada $\mathrm{Pd} / \mathrm{Fe} / \mathrm{Ir}(111)$. Para essa análise, consideraremos, em princípio, apenas as interações entre pares Co-Co (mesma camada), já que estas são as mais proeminentes. A Figura 5.15(a) mostra o valor médio do módulo do vetor $\left|\mathbf{D}_{\mathrm{Co}-\mathrm{Co}}\right|$ entre pares de átomos Co-Co em função da distância interatômica. É possível notar que, embora haja uma tendência geral de diminuição no valor encontrado de $\left|\mathbf{D}_{\mathrm{Co}-\mathrm{Co}}\right|$ com o aumento da separação entre os átomos de Co, existem dois "picos" nos quais a interação é maior quando comparada aos acoplamentos médios de vizinhanças adjacentes. Note-se também a forte interação média de DM entre primeiros vizinhos de Co, de $\left|\mathbf{D}_{\mathrm{Co}-\mathrm{Co}}\right|=0,57 \mathrm{meV}$ $\left(\sim 65 \%\right.$ do valor encontrado para as interações Fe-Fe). Isso resulta em uma relação $\frac{|\mathbf{D}|}{\mathcal{J}} \sim$ 0,042, ainda maior do que a obtida para o sistema similar de $\mathrm{Pd} / \mathrm{Fe} / \mathrm{Ir}(111)$. Ademais, calculando a densidade $D$ do acoplamento efetivo de DM, exatamente na forma descrita pela Eq. 5.2, encontramos para o $\mathrm{Pd} / \mathrm{Co} / \mathrm{Ir}(111)$ o inesperado valor de $D \sim 2,87 \mathrm{~mJ} / \mathrm{m}^{2}$, cerca de $66 \%$ do obtido para o $\mathrm{Pd} / \mathrm{Fe} / \operatorname{Ir}(111)\left(D \sim 4,36 \mathrm{~mJ} / \mathrm{m}^{2}\right)$. Em princípio, e considerando a proximidade entre as propriedades observadas para ambas as superfícies, esses resultados indicam uma forte tendência a favor da emergência de configurações nãocolineares de spin no estado fundamental do $\mathrm{Pd} / \mathrm{Co} / \mathrm{Ir}(111)$, e inclusive skyrmions em condições propícias, em acordo com as discussões feitas na referência [91]. 

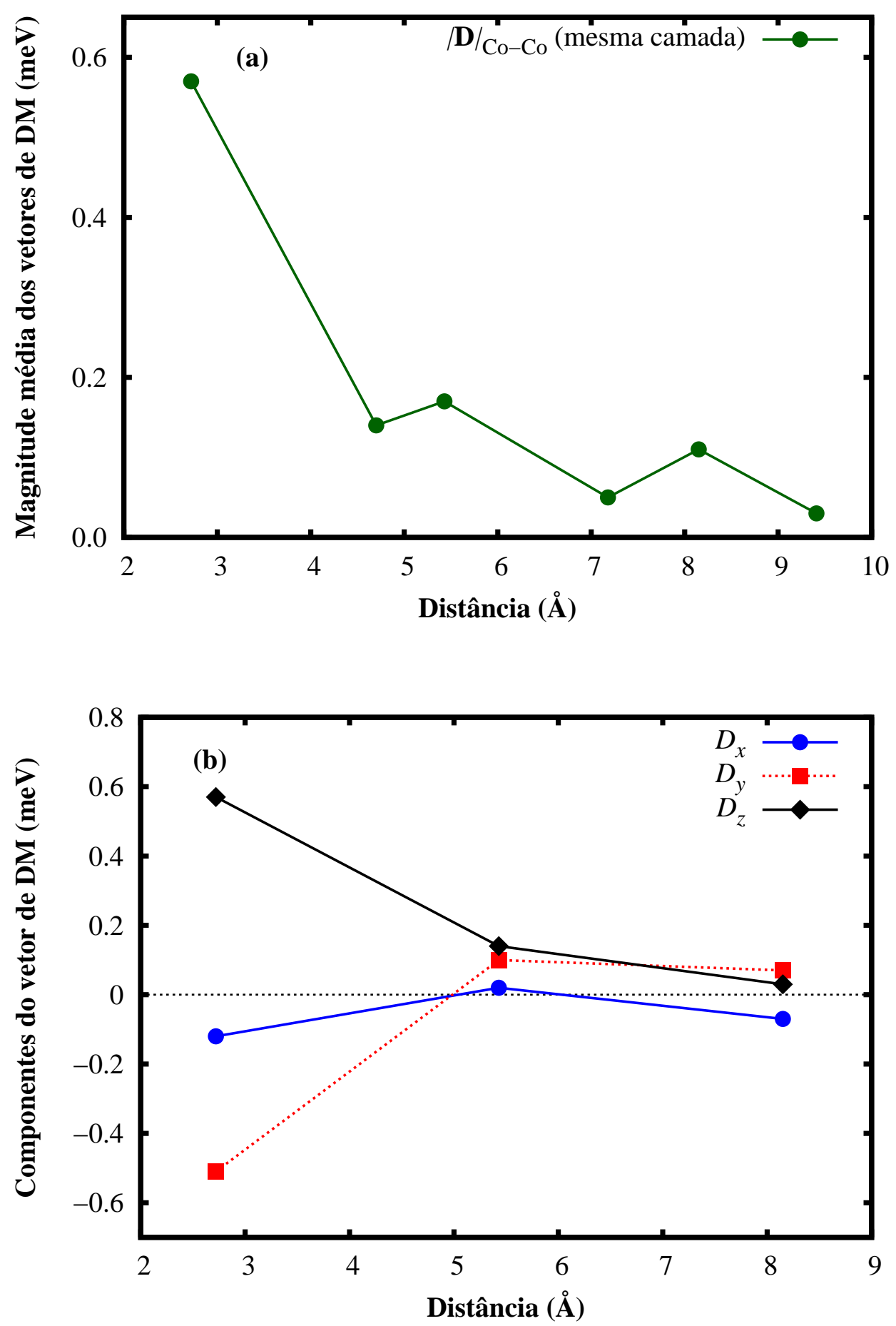

Figura 5.15: Resultados para: (a) valores médios das magnitudes $\left|\mathbf{D}_{\mathrm{Co}-\mathrm{Co}}\right|$ entre pares de átomos Co-Co, em função da distância interatômica; (b) componentes $D_{x}$, $D_{y}$, e $D_{z}$ para a interação de DM entre pares Co-Co vizinhos, na direção [101], em função da distância interatômica.

Por sua vez, as componentes $D_{x}, D_{y}$, e $D_{z}$ do vetor de DM entre pares de Co-Co vizinhos $\left(\mathbf{D}_{\mathrm{Co}-\mathrm{Co}}\right)$, avaliados para a direção in-plane [101], são apresentados na Fig. $\mathbf{5 . 1 5}$ (b). 
Nota-se, particularmente para $D_{x}$, que um comportamento oscilatório é observado como função da distância de separação - embora a contribuição dessa componente para a interação de primeiros vizinhos seja pequena em relação à atribuída a $D_{y}$ e $D_{z}$; a lista completa das componentes de cada interação de DM entre vizinhos mais próximos, seguindo a numeração definida na Fig. 5.5, é apresentada na Tabela 5.5. Analisando esses resultados encontramos que, ao invés de vetores localizados quase no plano da camada, os acoplamentos entre pares de primeiros vizinhos de Co-Co são essencialmente conduzidos por vetores $\mathbf{D}$ localizados quase fora do plano (out-of-plane). De fato, o ângulo médio entre os vetores $\mathbf{D}_{\mathrm{Co}-\text { Co }}$ para a primeira vizinhança e a superfície de $\operatorname{Ir}(111)$ é de aproximadamente $\bar{\phi} \sim 72^{\circ}$. Ao se afastar da primeira vizinhança, esse ângulo médio varia de $\bar{\phi} \sim 1^{\circ}$ (na segunda vizinhança) para $\bar{\phi} \sim 32^{\circ}$ (na sexta vizinhança). Portanto, uma orientação inversa de $\mathbf{D}_{\mathrm{Co}-\text { Co }}$ é verificada para primeiros e segundos vizinhos, já que os vetores de DM passam de uma orientação out-of-plane para in-plane com o aumento da distância entre os pares de átomos Co-Co. Ainda pela Tabela 5.5, é importante destacar que as mesmas equivalências entre os pares opostos $(1,2) \leftrightarrow-(1,5),(1,3) \leftrightarrow-(1,6)$, e $(1,4) \leftrightarrow-(1,7)$ são mantidas, respeitando, assim, a simetria $C_{3 h}$ da monocamada de Co. Entretanto, ao contrário do que se observa para o sistema $\mathrm{Pd} / \mathrm{Fe} / \mathrm{Ir}(111$ ) (Tabela 5.2), no caso do $\mathrm{Pd} / \mathrm{Co} / \operatorname{Ir}(111)$ as componentes dos pares $(1,2)$ e $(1,5)$ são significativamente reduzidas em comparação com algumas outras, originando pequenas magnitudes $|\mathbf{D}|$ para esses pares em relação aos demais.

Tabela 5.5: Componentes dos vetores de DM $\left(\mathbf{D}_{\mathrm{Co}-\mathrm{Co}}\right)$, expressos em meV, para as interações entre primeiros vizinhos de pares Co-Co. A numeração segue a definição da Fig. 5.5.

\begin{tabular}{rrrr}
\hline$(i, j)$ & \multicolumn{1}{c}{$D_{x}$} & \multicolumn{1}{c}{$D_{y}$} & \multicolumn{1}{c}{$D_{z}$} \\
\hline \hline$(1,2)$ & 0,10 & 0,09 & 0,10 \\
$(1,3)$ & $-0,12$ & $-0,51$ & $-0,57$ \\
$(1,4)$ & 0,51 & 0,12 & 0,57 \\
$(1,5)$ & $-0,09$ & $-0,10$ & $-0,10$ \\
$(1,6)$ & 0,12 & 0,51 & 0,57 \\
$(1,7)$ & $-0,51$ & $-0,12$ & $-0,57$ \\
\hline
\end{tabular}

A Figura 5.16, a seguir, apresenta as direções calculadas dos vetores de DM para as interações de primeiros (Fig. 5.16(a)) e segundos (Fig. 5.16(b)) vizinhos, sem considerar as diferentes magnitudes. Assim como na Fig. 5.6, esses vetores são representados no sítio do segundo átomo do par, assumindo que o primeiro átomo é, em todos os casos, o Co central. 
(a)

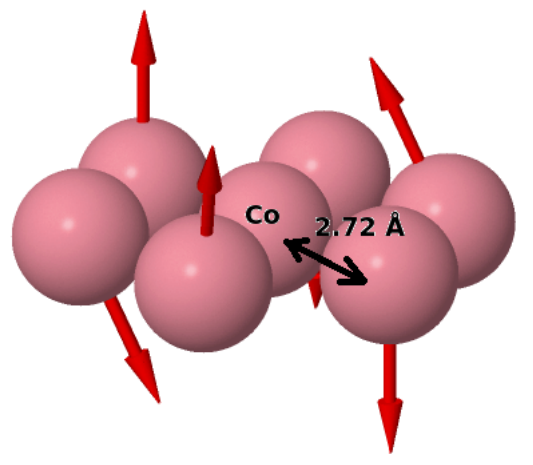

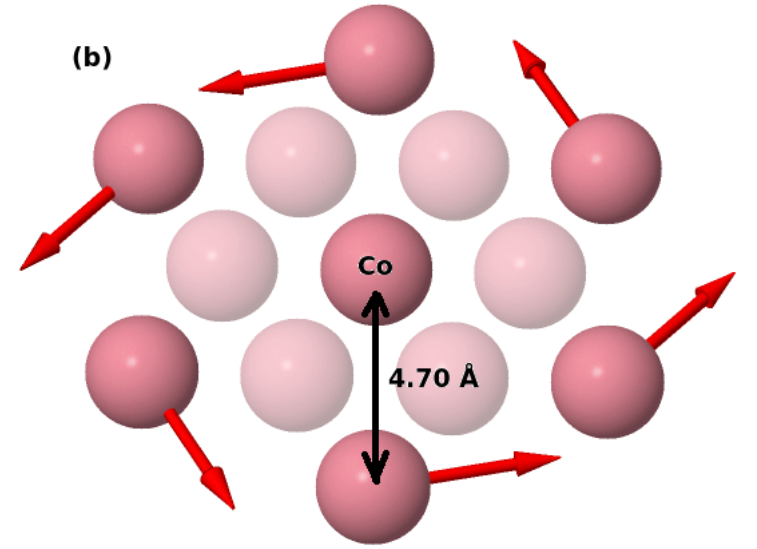

Figura 5.16: Direções calculadas dos vetores $\mathbf{D}_{\mathrm{Co}-\mathrm{Co}}$ (em vermelho) para interações de: (a) primeiros vizinhos; (b) segundos vizinhos com o átomo de Co central (indicado). As esferas em rosa representam os átomos de Co.

Embora o forte acoplamento de DM observado para os pares de Co-Co no sistema $\mathrm{Pd} / \mathrm{Co} / \mathrm{Ir}(111)$ indique que há condições necessárias ao surgimento de texturas complexas de spin (ou ao menos pequenos desvios do estado colinear), a configuração final obtida ao se realizar cálculos não-colineares é, em todos os casos investigados, preferencialmente FM com um eixo de fácil magnetização normal à superfície de $\operatorname{Ir}(111)$; de fato, a energia de anisotropia magnética, aqui assumida como sendo uniaxial, é de aproximadamente $E_{\mathrm{MAE}} \sim-0,03 \mathrm{meV}$ em favor da magnetização perpendicular ao plano de superfície, obtida utilizando a relação de Bruno [119] (Eq. 2.21), onde $\xi_{\mathrm{Co}}=142,2 \mathrm{meV}^{1}$. A mesma configuração FM final é mantida quando não se consideram os efeitos do acoplamento spinórbita nos cálculos não-colineares, produzindo sempre soluções colineares de mais baixa energia. Em outras palavras, diferentemente do obtido para a bicamada de $\mathrm{Pd} / \mathrm{Fe} / \mathrm{Ir}(111)$, não se atingem mínimos locais metaestáveis não-colineares, e sim apenas configurações FM dos momentos magnéticos de spin. Esse resultado está de acordo com observações experimentais anteriores, à baixas temperaturas $(T=8,0 \pm 0,5 \mathrm{~K})$, para esse sistema [61]. De uma maneira mais geral, o estado fundamental FM observado para a bicamada de $\mathrm{Pd} / \mathrm{Co} / \operatorname{Ir}(111)$ sugere que o aumento no número médio de elétrons de valência por sítio na camada magnética conduz o sistema de um estado de mais baixa energia descrito por uma espiral de spin (Fig. 5.8), isto é, não-colinear, para um estado FM (colinear). A mesma tendência já havia sido observada em estudos prévios [56, 191], entretanto considerando mudanças no número de elétrons de valência na camada de revestimento ${ }^{2}$ ao invés de variações na estrutura eletrônica de valência da própria camada magnética.

\footnotetext{
${ }^{1} \mathrm{O}$ eixo de fácil magnetização out-of-plane encontrado está de acordo com as observações experimentais para a bicamada de $\mathrm{Pd} / \mathrm{Co} / \operatorname{Ir}(111)$ [61].

${ }^{2}$ Nos casos investigados no presente trabalho, a camada de revestimento é sempre composta por Pd.
} 
Como esperado, os cálculos de dinâmica de spins para a monocamada de Co utilizando os parâmetros obtidos via primeiros princípios também resultaram em uma configuração FM para $T_{\text {final }}=10^{-4} \mathrm{~K}\left(T_{\text {final }} \sim 0 \mathrm{~K}\right)$, tanto na ausência de campo $\mathbf{B}$ externo quanto para $|\mathbf{B}| \neq 0$, aplicado em diferentes direções. Nesse sentido, é importante destacar que, para testes realizados nos quais aumentamos artificialmente em até $100 \%$ as componentes dos vetores de DM obtidas pelos cálculos ab initio, não foram observadas alterações na textura magnética (nem com a influência de campo externo, seja in-plane ou out-of-plane), permanecendo o estado fundamental descrito por um arranjo colinear de momentos de spin. O aumento de temperatura da simulação conduz, naturalmente, à fase paramagnética, sendo a temperatura crítica (ou de Curie), obtida por teoria de campo médio, de $T_{C}=708,3$ K. Baseado nas conclusões anteriores para a bicamada de $\mathrm{Pd} / \mathrm{Fe} / \mathrm{Ir}(111)$ (seção 5.1), é possível estabelecer as seguintes inferências: $(i)$ a inexistência de frustração magnética apreciável no sistema $\mathrm{Pd} / \mathrm{Co} / \mathrm{Ir}(111)$, tendo como origem as interações de troca, constitui uma diferença fundamental entre as duas bicamadas investigadas, de forma que o custo energético para se produzir soluções não-colineares na monocamada de Co é bastante elevado e não atingido pela interação com o campo magnético externo; e (ii) apesar do forte acoplamento de DM encontrado, as interações de troca são determinantes para o comportamento magnético do sistema $\mathrm{Pd} / \mathrm{Co} / \mathrm{Ir}(111)$.

Assim, o termo de acoplamento de DM desaparece, tornando as interações do tipo de Heisenberg como as principais entre os spins do sistema. Discutiremos esse efeito em detalhes na seção 5.3, a seguir, tendo como base a comparação entre as bicamadas de $\mathrm{Pd} / \mathrm{Fe} / \operatorname{Ir}(111)$ e $\mathrm{Pd} / \mathrm{Co} / \operatorname{Ir}(111)$.

\subsection{Comparação entre as bicamadas}

A fim de analisarmos de maneira minuciosa a comparação entre as bicamadas de $\mathrm{Pd} / \mathrm{Fe} / \mathrm{Ir}(111)$ e $\mathrm{Pd} / \mathrm{Co} / \mathrm{Ir}(111)$, é útil relembrarmos que, similarmente à definição associada às interações de troca $\left(\mathcal{J}_{i j}\right)[34,154]$, a convenção utilizada para o cálculo do termo de energia vinculado aos acoplamentos de DM é escrito na forma

$$
-\sum_{(i, j)} \mathbf{D}_{i j} \cdot\left(\hat{\mathbf{e}}_{i} \times \hat{\mathbf{e}}_{j}\right)
$$

para todos os pares atômicos $(i, j)$ não-equivalentes, sendo que $\hat{\mathbf{e}}_{i}$ representa o vetor unitário paralelo à direção do momento magnético local no sítio $i$. Portanto, à primeira 
vista, é evidente que, além de uma elevada magnitude de interação $\left|\mathbf{D}_{i j}\right|$, o ângulo $\varphi$ entre os vetores $\mathbf{D}_{i j}$ e $\left(\hat{\mathbf{e}}_{i} \times \hat{\mathbf{e}}_{j}\right)$ de cada acoplamento é também muito importante para se realizar a análise da estabilidade de texturas de spin que desviam do estado colinear. Se $\varphi$ é próximo de $90^{\circ}$, por exemplo, então $\cos \varphi \sim 0$, tornando desprezível o termo de energia definido na Eq. $\mathbf{5 . 4}$ em relação ao termo isotrópico de Heisenberg na Hamiltoniana de spin. Da mesma forma, os ângulos $\theta$ entre os momentos de spin locais (representados pelos vetores unitários $\hat{\mathbf{e}}_{i}$ e $\hat{\mathbf{e}}_{j}$ ), e o ângulo $\phi$ entre o vetor $\mathbf{D}_{i j}$ e a superfície de $\operatorname{Ir}(111)$ são fundamentais para se investigar as origens físicas para a emergência de soluções nãocolineares de mais baixa energia na bicamada de $\mathrm{Pd} / \mathrm{Fe} / \operatorname{Ir}(111)$. Os ângulos $\theta, \varphi$, e $\phi$ estão esquematicamente definidos na Figura 5.17.
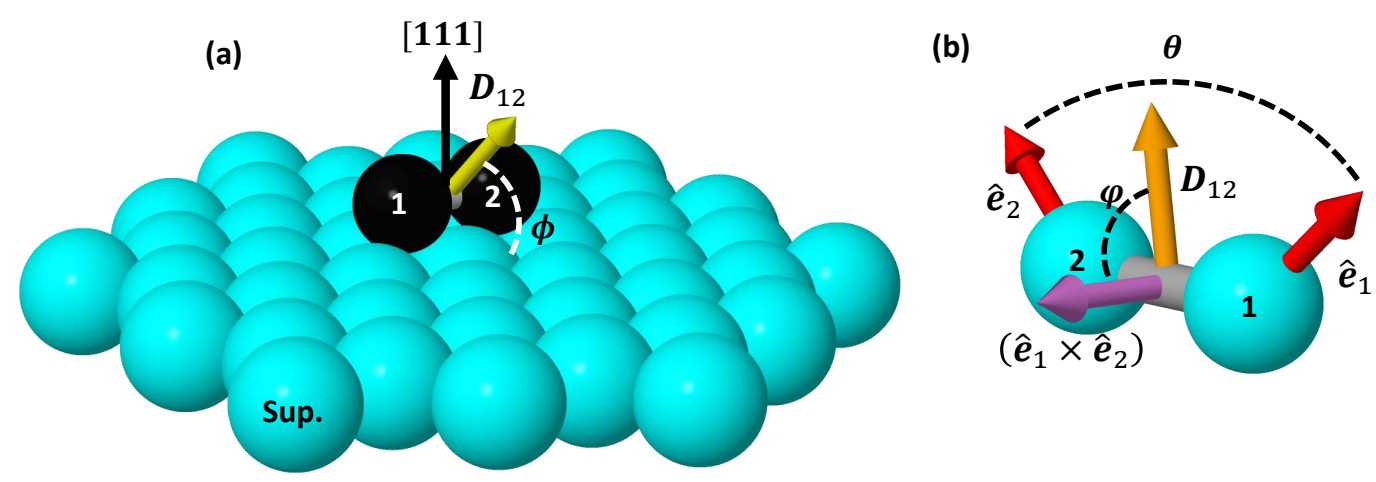

Figura 5.17: Definição dos ângulos: $(a) \phi$, entre o vetor $\mathbf{D}_{i j}$ e a superfície de $\operatorname{Ir}(111)$; (b) $\theta$ e $\varphi$, entre as direções locais dos momentos de spin nos sítios $i$ e $j$, denotadas por $\hat{\mathbf{e}}_{i}$ e $\hat{\mathbf{e}}_{j}$, e entre o vetor $\mathbf{D}_{i j}$ e o produto vetorial $\left(\hat{\mathbf{e}}_{i} \times \hat{\mathbf{e}}_{j}\right)$, respectivamente. As esferas em azul claro e na cor preta representam átomos genéricos de ambos os sistemas investigados.

O ângulo médio calculado $\bar{\varphi}$ entre $\mathbf{D}_{i j}$ e $\left(\hat{\mathbf{e}}_{i} \times \hat{\mathbf{e}}_{j}\right)$, bem como os ângulos médios $\bar{\theta}$ e $\bar{\phi}$ e a magnitude do termo de DM na Hamiltoniana (Eq. 5.4) para as interações Fe-Fe e CoCo nos sistemas de $\mathrm{Pd} / \mathrm{Fe} / \mathrm{Ir}(111)$ e $\mathrm{Pd} / \mathrm{Co} / \mathrm{Ir}(111)$, respectivamente, estão apresentados na Tabela 5.6, a seguir. Nela, cada uma das entradas para os termos de DM pressupõem o valor absoluto do somatório definido na Eq. 5.4, para todos os pares atômicos nãoequivalentes $(i, j)$ pertencentes à vizinhança correspondente. Por sua vez, note que as magnitudes médias $\left|\mathbf{D}_{\mathrm{Fe}-\mathrm{Fe}}\right|$ e $\left|\mathbf{D}_{\mathrm{Co}-\mathrm{Co}}\right|$, também listadas na Tabela 5.6, são as mesmas traçadas nos gráficos das Figs. 5.4(a) e 5.15(a), nesta ordem. 
Tabela 5.6: Ângulos médios $\bar{\varphi}, \bar{\phi}$, e $\bar{\theta}$ (esquematicamente definidos na Fig. 5.17), distância entre pares atômicos (em $\AA$ ), termos de DM (Eq. 5.4), em meV, bem como a magnitude média dos vetores de DM (também em meV), calculados para os sistemas de $\mathrm{Pd} / \mathrm{Fe} / \operatorname{Ir}(111)$ e $\mathrm{Pd} / \mathrm{Co} / \operatorname{Ir}(111)$ nas interações entre átomos pertencentes às camadas magnéticas. Aqui, estendendo a notação já definida anteriormente, NN representa os primeiros vizinhos, NNN os segundos vizinhos, e $\mathrm{N}^{(n)}$ a $(n-1)$-ésima vizinhança.

\begin{tabular}{ccccccc}
\hline \hline \multicolumn{7}{c}{$\mathbf{P d} / \mathbf{F e} / \mathbf{I r}(\mathbf{1 1 1})$} \\
\hline Vizinhança & Distância & $\left|\mathbf{D}_{\mathrm{Fe}-\mathrm{Fe}}\right|$ & $\bar{\varphi}$ & $\bar{\phi}$ & $\bar{\theta}$ & Termo de DM \\
\hline $\mathbf{N N}$ & 2,72 & 0,87 & $52^{\circ}$ & $8^{\circ}$ & $69^{\circ}$ & 0,58 \\
$\mathbf{N N N}$ & 4,70 & 0,24 & $66^{\circ}$ & $43^{\circ}$ & $44^{\circ}$ & 0,35 \\
$\mathbf{N}^{(4)}$ & 5,43 & 0,17 & $46^{\circ}$ & $24^{\circ}$ & $20^{\circ}$ & 0,09 \\
$\mathbf{N}^{(5)}$ & 7,18 & 0,14 & $56^{\circ}$ & $26^{\circ}$ & $42^{\circ}$ & 0,36 \\
$\mathbf{N}^{(6)}$ & 8,15 & 0,24 & $60^{\circ}$ & $38^{\circ}$ & $66^{\circ}$ & 0,63 \\
$\mathbf{N}^{(7)}$ & 9,41 & 0,06 & $60^{\circ}$ & $30^{\circ}$ & $49^{\circ}$ & 0,11 \\
\hline \hline \multicolumn{7}{c}{$\mathbf{P d} / \mathbf{C o} / \mathbf{I r}(\mathbf{1 1 1})$} \\
\hline Vizinhança & Distância & $\left|\mathbf{D}_{\mathrm{Co}-\mathrm{Co}}\right|$ & $\bar{\varphi}$ & $\phi$ & $\theta$ & Termo de DM \\
\hline $\mathbf{N N}$ & 2,72 & 0,57 & $80^{\circ}$ & $72^{\circ}$ & $<1^{\circ}$ & $\sim 10^{-3}$ \\
$\mathbf{N N N}^{-3}$ & 4,70 & 0,14 & $37^{\circ}$ & $1^{\circ}$ & $<1^{\circ}$ & $\sim 10^{-3}$ \\
$\mathbf{N}^{(4)}$ & 5,43 & 0,17 & $53^{\circ}$ & $47^{\circ}$ & $1^{\circ}$ & $<10^{-3}$ \\
$\mathbf{N}^{(5)}$ & 7,18 & 0,05 & $48^{\circ}$ & $28^{\circ}$ & $1^{\circ}$ & $<10^{-3}$ \\
$\mathbf{N}^{(6)}$ & 8,15 & 0,11 & $70^{\circ}$ & $26^{\circ}$ & $2^{\circ}$ & $<10^{-3}$ \\
$\mathbf{N}^{(7)}$ & 9,41 & 0,03 & $63^{\circ}$ & $32^{\circ}$ & $2^{\circ}$ & $<10^{-3}$ \\
\hline
\end{tabular}

A partir desses resultados, é possível extrair algumas conclusões importantes. Em primeiro lugar, verifica-se que os ângulos médios $\bar{\varphi}$ entre $\mathbf{D}_{i j}$ e $\left(\hat{\mathbf{e}}_{i} \times \hat{\mathbf{e}}_{j}\right)$ não são sempre próximos de $90^{\circ}$ para as interações anisotrópicas (de DM) entre pares de Co-Co no sistema $\mathrm{Pd} / \mathrm{Co} / \operatorname{Ir}(111)$, exceto para o acoplamento de primeiros vizinhos, em que se obtém $\bar{\varphi} \sim$ $80^{\circ}$. De fato, se observam ângulos $\bar{\varphi}$ até algumas vezes menores do que os obtidos para a mesma vizinhança nas interações entre pares $\mathrm{Fe}-\mathrm{Fe}$ na bicamada de $\mathrm{Pd} / \mathrm{Fe} / \mathrm{Ir}(111)$. Então qual seria a causa para a significativa atenuação dos termos de DM no caso da bicamada de $\mathrm{Pd} / \mathrm{Co} / \mathrm{Ir}(111)$ ? Podemos entender os pequenos valores de energia para os termos de DM como resultado e consequência do próprio alinhamento FM entre os spins do Co, o que acarreta em $\left|\left(\hat{\mathbf{e}}_{i} \times \hat{\mathbf{e}}_{j}\right)\right| \sim \operatorname{sen}(\theta) \sim 0$, praticamente anulando qualquer produto escalar com os vetores $\mathbf{D}_{i j}$, por maiores que sejam. Assim, os termos de DM para as interações Co-Co quase desaparecem, tornando o magnetismo em escala nanométrica predominantemente conduzido pelos acoplamentos isotrópicos de troca $\left(\mathcal{J}_{i j}\right)$. Além disso, é importante ressaltar que, embora exista uma forte interação de DM sobretudo entre pares de primeiros vizinhos, o vetor $\mathbf{D}_{\mathrm{Co}-\text { Co }}$ é quase perpendicular à superfície de $\operatorname{Ir}(111)$, o que favorece ainda mais uma configuração colinear de menor energia entre os momentos de spin dos átomos de Co, haja vista que o eixo de fácil magnetização é normal à superfície de $\operatorname{Ir}(111)$, propiciando um alinhamento dos spins orientado para fora do plano (out-of- 
plane).

No caso da bicamada de $\mathrm{Pd} / \mathrm{Fe} / \mathrm{Ir}(111)$, a discussão torna-se um pouco mais complexa. Em princípio, nota-se que os ângulos médios $\bar{\theta}$ entre os momentos de spin locais são muito maiores que os encontrados para o sistema $\mathrm{Pd} / \mathrm{Co} / \mathrm{Ir}(111)$, e, portanto, o produto vetorial $\left|\left(\hat{\mathbf{e}}_{i} \times \hat{\mathbf{e}}_{j}\right)\right| \sim \operatorname{sen}(\theta)$ não tem o mesmo efeito de anular o termo de DM associado às interações entre pares de vizinhos Fe-Fe. Conforme mencionado anteriormente, as soluções não-colineares obtidas sem considerar o efeito do acoplamento spin-órbita no processo autoconsistente mostraram-se muito próximas em energia à solução colinear do sistema, caracterizando, portanto, estados metaestáveis. Isso significa que, diferentemente do que ocorre com o sistema $\mathrm{Pd} / \mathrm{Co} / \mathrm{Ir}(111)$, no caso da camada de Fe a frustração magnética conduzida sobretudo pela interação AFM entre pares Fe-Fe terceiros vizinhos desempenha um papel importante na origem da não-colinearidade dos spins, favorecendo ângulos $\bar{\theta}_{\mathrm{Fe}-\mathrm{Fe}} \gg 0^{\circ}$. Na mesma linha de raciocínio, Okubo et al. [192] demonstraram a relevância da frustração magnética na formação de texturas complexas de spin em uma rede bidimensional triangular (simetria $C_{3}$ ), podendo inclusive ser responsável pela emergência de (anti-)skyrmions quando o sistema é submetido a certas magnitudes de campo magnético externo. Nesse trabalho, é interessante notar que soluções complexas aparecem quando $\frac{\left|\mathcal{J}_{3}\right|}{\mathcal{J}_{1}}>25 \%$, sendo $\mathcal{J}_{3}$ a interação entre terceiros vizinhos e $\mathcal{J}_{1}$ a troca entre primeiros vizinhos; nesse sentido, cabe lembrar que, a partir de nossos cálculos, obtivemos $\frac{\left|\mathcal{J}_{3}\right|}{\mathcal{J}_{1}} \sim 17 \%$ para pares de átomos Fe-Fe, um valor muito próximo do limite mínimo previsto na referência [192] para a emergência de texturas não-colineares de spin. Também cabe mencionar que a referência [191] discute a importância da interação de troca efetiva entre primeiros vizinhos para a determinação de um estado de menor energia caracterizado por uma espiral de spin, a partir da análise de bicamadas metálicas semelhantes às investigadas no presente trabalho.

Concomitantemente ao efeito das interações de troca no sistema, um sentido de rotação nas direções locais dos spins é introduzido como consequência do elevado acoplamento spin-órbita do Ir (cerca de uma ordem de grandeza maior que o do Fe, e $\sim 2,5$ vezes maior que o do $\mathrm{Pd}$ ), que, em conjunto com a quebra de simetria de inversão, naturalmente provocada pelo fato de o sistema ser uma superfície não-homogênea (i. e., formado por múltiplas camadas de metais de transição distintos), leva a uma forte interação de DM entre os pares de átomos Fe-Fe. Nesse sentido, é importante notar que a interação de DM mais significativa, entre primeiros vizinhos, é caracterizada por vetores $\mathbf{D}_{i j}$ quase paralelos à superfície de $\operatorname{Ir}(111)$, favorecendo uma rotação local dos momentos de spin na direção in-plane, e influenciando, assim, diretamente na emergência de um magnetismo não-colinear. 
Além desse efeito conjunto, algumas interessantes características da bicamada de $\mathrm{Pd} / \mathrm{Fe} / \mathrm{Ir}(111)$ podem ser destacadas. Em primeiro lugar, nota-se que para a quinta vizinhança $\left(\mathrm{N}^{(6)}\right)$, localizados à uma distância significativa de $\sim 8,15 \AA$ do átomo central de Fe, o termo de DM é ainda maior que o calculado para a contribuição de segundos vizinhos (NNN). Isso evidencia uma influência de longo alcance das interações de DM, mediadas pelo hopping dos elétrons de condução através das bandas estendidas $5 d$ do Ir. A expressiva contribuição do termo de DM advinda das interações entre pares de quintos vizinhos Fe-Fe apresenta duas razões simples: $(i)$ o somatório da Eq. 5.4 é definido para 12 pares de átomos distintos, ao invés de 6 pares definidos para todos os outros casos; e (ii) os resultados obtidos para $\left|\mathbf{D}_{\mathrm{Fe}-\mathrm{Fe}}\right|$ não seguem uma diminuição monotônica, mas apresenta, como pôde ser observado na Fig. 5.4(a), um valor aumentado para a interação com $5^{\text {os }}$ vizinhos em relação às contribuições das bases de vizinhos mais próximos. Conforme indicado pela referência [54], a simulação de uma rede de skyrmions na bicamada de $\mathrm{Pd} / \mathrm{Fe} / \mathrm{Ir}(111)$ como solução da equação de LLG só foi possível quando considerado um grande número de vizinhos (ou seja, um extenso raio de interação), demonstrando indiretamente a significativa contribuição de longo alcance das interações de troca e de DM. Por sua vez, a segunda característica a ser destacada é a de que o termo de DM para a terceira vizinhança, a mesma na qual os átomos de Fe apresentam um acoplamento AFM com o Fe de referência, exibe o menor valor dentre todas as outras contribuições, sendo inclusive reduzida em relação à vizinhanças bem mais distantes. Esse efeito pode ser atribuído aos ângulos pequenos $\theta_{i j}$ entre os momentos de spin de sítios $i$ e $j$ distantes um do outro por um número inteiro ou semi-inteiro de comprimentos de onda $(\lambda)$, característico de um estado de espiral de spin: se iniciarmos, por exemplo, com um spin perpendicular à superfície (direção [111]), em determinada distância $\left(\frac{\lambda}{2}\right)$ existirá um outro spin na mesma direção, porém em sentido contrário. Nessa distância, o produto $\left(\hat{\mathbf{e}}_{i} \times \hat{\mathbf{e}}_{j}\right)$ é zero, desaparecendo completamente com o termo de DM correspondente. Entretanto, devemos lembrar que, tal como mostrado na Fig. 5.7, os spins não formam exatamente uma configuração em espiral como resultado do cálculo de primeiros princípios ${ }^{1}$, mas verificam-se regiões ( $3^{\text {os }}$ vizinhos) nas quais os spins, de fato, estão quase na mesma direção do spin do átomo central (de referência), diminuindo significativamente o valor de $\left|\left(\hat{\mathbf{e}}_{i} \times \hat{\mathbf{e}}_{j}\right)\right|$.

Considerando a relevância das interações de troca (isotrópicas) na formação de um estado fundamental não-colinear na bicamada de $\mathrm{Pd} / \mathrm{Fe} / \mathrm{Ir}(111)$ (e de uma rede de skyrmions nesse sistema quando aplicado um campo magnético de magnitude apropriada), estudaremos agora a natureza e o comportamento dessas interações em ambos os sistemas investigados. Uma proposta, que já foi utilizada em trabalhos anteriores [32, 36, 193], é

\footnotetext{
${ }^{1}$ Isso se deve, provavelmente, à aproximação de tratarmos uma superfície homogênea, infinita em duas direções, como uma ilha finita no espaço real.
} 
analisar a estrutura da curva $\mathcal{J}_{i j}(E)$, isto é, do parâmetro de troca entre os sítios $i$ e $j$ como função da energia. Nesse caso, devemos recordar que o valor com significado físico para essa interação é aquele avaliado na energia de Fermi, ou seja, $\mathcal{J}_{i j}=\mathcal{J}_{i j}\left(E_{F}\right)$. A Figura 5.18, abaixo, mostra os resultados obtidos para $\mathcal{J}_{\mathrm{Fe}-\mathrm{Fe}}(E)$ e $\mathcal{J}_{\mathrm{Co}-\mathrm{Co}}(E)$, de primeiros a terceiros vizinhos. Nota-se que ambas as curvas, para as interações entre pares Fe-Fe e Co-Co, possuem perfis muito semelhantes entre si, e apresentam um comportamento oscilatório dependente da energia. Isso sugere que a localização da energia de Fermi é um fator determinante na presença ou não de valores negativos de $\mathcal{J}_{i j}$, indiretamente influenciando na existência de frustração magnética no sistema. Como se observa na Fig. 5.18(a), o valor calculado de $E_{F}$ para a bicamada de $\mathrm{Pd} / \mathrm{Fe} / \mathrm{Ir}(111)$ encontra-se em uma região na qual a interação de troca para terceiros vizinhos Fe-Fe é negativa, ao contrário do que ocorre com a bicamada de $\mathrm{Pd} / \mathrm{Co} / \mathrm{Ir}(111)$ (Fig. 5.18(b)), em particular devido ao menor preenchimento da banda $d$ do Fe (menor número de estados ocupados). Neste caso, portanto, é possível fazer uma análise análoga ao modelo de banda rígida para explicar os valores encontrados de $\mathcal{J}_{i j}$ em ambos os sistemas. 

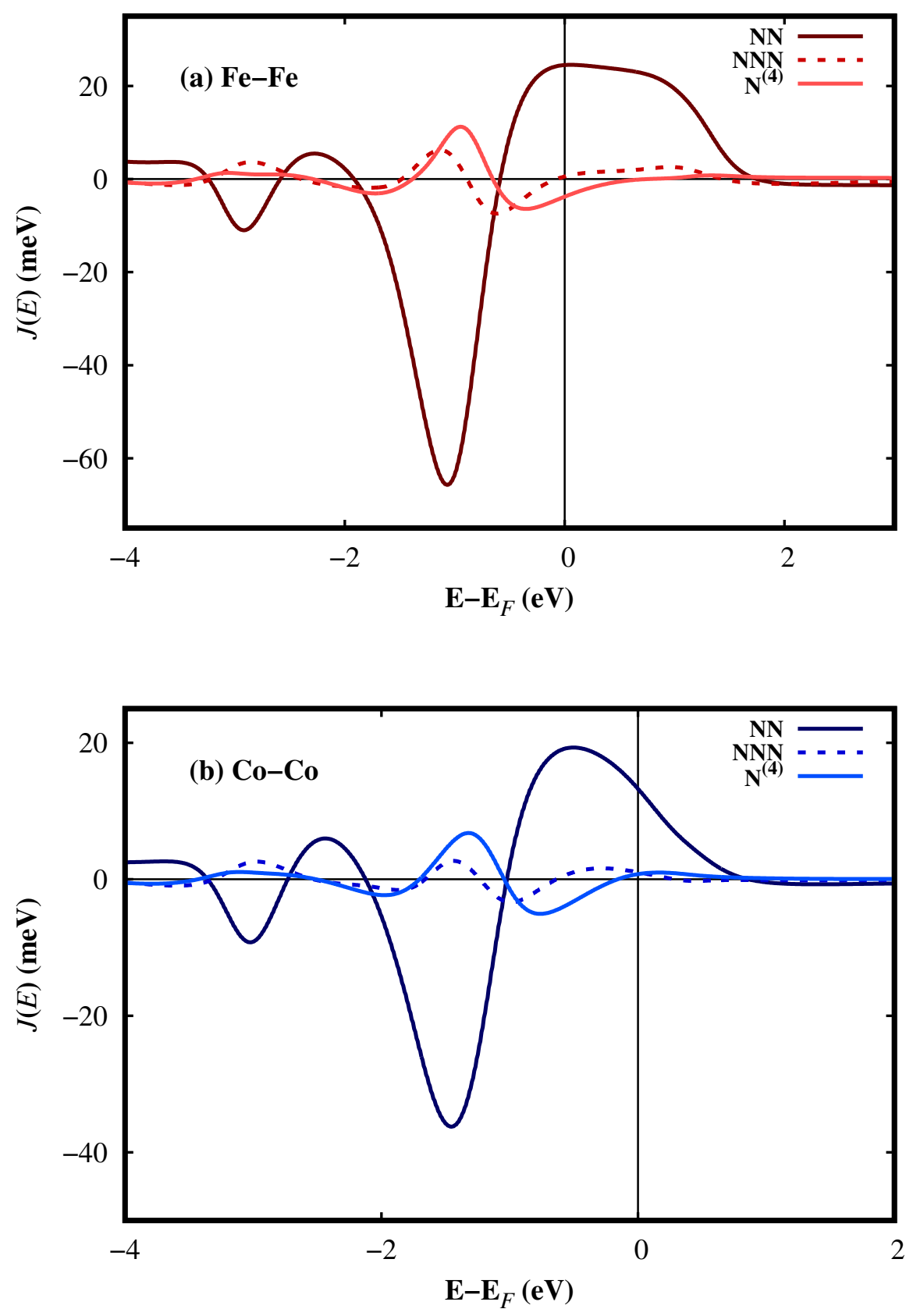

Figura 5.18: Parâmetros de troca $\mathcal{J}_{i j}$ como função da energia entre primeiros $(\mathrm{NN})$, segundos $(\mathrm{NNN})$, e terceiros $\left(\mathrm{N}^{(4)}\right)$ vizinhos de: $(a)$ pares de átomos $\mathrm{Fe}-\mathrm{Fe}$, na bicamada de $\mathrm{Pd} / \mathrm{Fe} / \operatorname{Ir}(111)$; e $(b)$ pares de átomos Co-Co, na bicamada de $\mathrm{Pd} / \mathrm{Co} / \mathrm{Ir}(111) . E_{F}$ é a energia de Fermi correspondente a cada sistema.

Análise similar pode ser realizada com as componentes dos vetores $\mathbf{D}_{i j}$ como função da energia para ambos os sistemas de $\mathrm{Pd} / \mathrm{Fe} / \mathrm{Ir}(111)$ e $\mathrm{Pd} / \mathrm{Co} / \mathrm{Ir}(111)$. Os gráficos resultantes são apresentados na Fig. 5.19, para uma mesma sequência de vizinhos em ambas as multicamadas. É importante destacar que a implementação em FORTRAN90, que tornou possível obter $\mathbf{D}_{i j}(E)$, é parte dos resultados desta tese. 


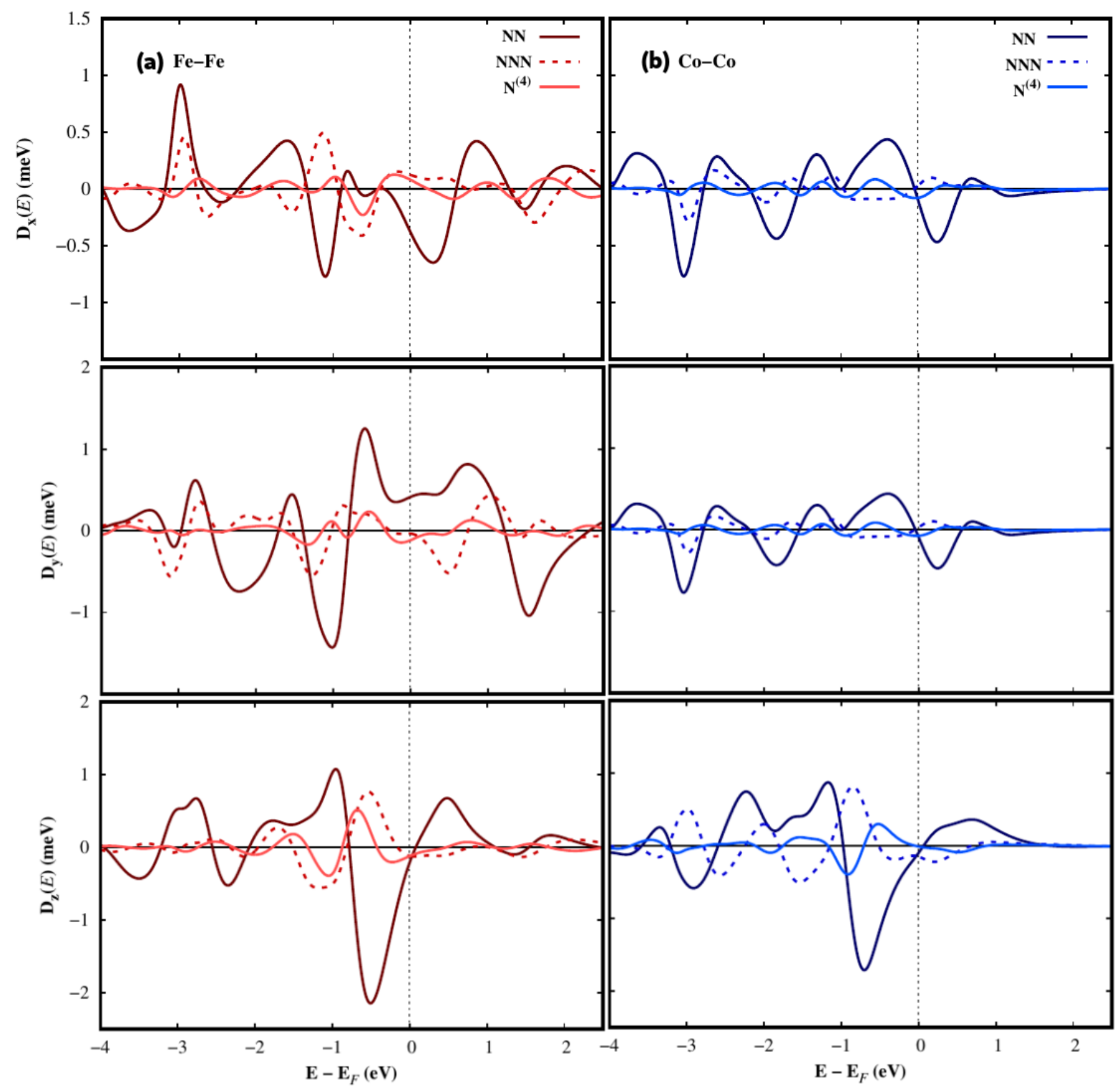

Figura 5.19: Componentes dos vetores de interação $\mathbf{D}_{i j}$ como função da energia entre primeiros $(\mathrm{NN})$, segundos $(\mathrm{NNN})$, e terceiros $\left(\mathrm{N}^{(4)}\right)$ vizinhos de: $(a)$ pares de átomos Fe-Fe, na bicamada de $\mathrm{Pd} / \mathrm{Fe} / \operatorname{Ir}(111)$; e (b) pares de átomos Co-Co, na bicamada de $\mathrm{Pd} / \mathrm{Co} / \operatorname{Ir}(111) . E_{F}$ é a energia de Fermi correspondente a cada sistema.

Analogamente ao verificado no caso de $\mathcal{J}_{i j}(E)$ (Fig. 5.18), nota-se curvas semelhantes entre as componentes $D_{x}, D_{y}$ e $D_{z}$ - em particular para $D_{z}$. Assim, para o caso das interações de DM, a localização de $E_{F}$ também se mostra um fator importante para a determinação do vetor $\mathbf{D}_{i j}$, e a existência ou não de valores negativos/positivos (i.e., na direção do vetor de interação de DM em relação à superfície).

Recentemente, Y. O. Kvashnin et al. [194] caracterizaram o estado fundamental FM do Fe bcc (bulk) investigando as interações on-site entre as representações irredutíveis $e_{g}$ (composta dos orbitais $d_{x^{2}-y^{2}}$ e $d_{3 z^{2}-r^{2}}$, bidegenerada) e $t_{2 g}$ (composta dos orbitais $d_{x y}$, 
$d_{x z}, d_{y z}$, tridegenerada), e suas contribuições para a composição de cada acoplamento de troca. Portanto, podemos aprofundar nossa investigação sobre a natureza das interações de troca analisando a decomposição de cada valor $\mathcal{J}_{i j}$ nas suas contribuições por orbital. Antes de prosseguirmos, porém, cabem aqui algumas observações. A primeira delas é que nos metais de transição o potencial de troca que atua nos estados $d$ é muito maior em comparação aos que atuam nos estados $s$ e $p$, produzindo um maior exchange splitting entre as bandas $d$ majoritárias e minoritárias. Assim, levando em conta o fato que o cálculo de $\mathcal{J}_{i j}$ no formalismo adotado neste trabalho [154] depende da quantidade $\delta_{i}(E)$, que tem unidades de energia e pode ser associada ao exchange splitting local no sítio $i$ (dependente da energia $E$ ), então é aceitável considerar os valores $\mathcal{J}_{i j}$ constituídos apenas das contribuições advindas dos orbitais $d$ (embora, na prática, esses orbitais já tenham certa influência $s-p$, já que o cálculo de primeiros princípios foi realizado inicialmente com todos os orbitais presentes). De fato, em conformidade com a referência [154], encontramos que as contribuições das interações entre orbitais $d-d$ correspondem a mais de $90 \%$ do valor total de $\mathcal{J}_{i j}$, em todos os casos investigados. A segunda observação é que, diferentemente do realizado na referência [194], nossos cálculos incluem o efeito do acoplamento spin-órbita na autoconsistência, e, portanto, não podemos mais separar os orbitais $d$ nas representações bi- e tridegenerada $e_{g}$ e $t_{2 g}$. Logo, tomamos como válida a seguinte relação:

$$
\mathcal{J}_{i j} \sim \sum_{\alpha, \beta} \mathcal{J}_{i j}^{d_{\alpha}-d_{\beta}}
$$

onde $\alpha, \beta=x y, y z, x z, x^{2}-y^{2}, 3 z^{2}-r^{2}$, e $\mathcal{J}_{i j}^{d_{x y}-d_{y z}}$, por exemplo, simboliza a contribuição da interação $\mathcal{J}_{i j}$ advinda do acoplamento on-site entre o orbital $d_{x y}$ do sítio $i$ e o orbital $d_{y z}$ do sítio $j$. Cabe ressaltar que, devido às variações na estrutura eletrônica local, as interações de troca entre sítios atômicos equidistantes não são necessariamente equivalentes em termos das contribuições orbitais, como veremos mais adiante.

Sendo assim, as decomposições das interações $\mathcal{J}_{i j}$ para ambos os pares de átomos Fe-Fe e Co-Co, de primeiros a terceiros vizinhos, são listadas na Tabela 5.7, na qual destacamos apenas as maiores contribuições de um total de 10 interações distintas entre orbitais $d-d$ (considerando que $\mathcal{J}_{i j}^{d_{\alpha}-d_{\beta}}=\mathcal{J}_{j i}^{d_{\beta}-d_{\alpha}}$ ). Analisando a Tabela 5.7, claramente se nota o papel decisivo que os termos denominados mistos $\left(d_{\alpha}-d_{\beta}\right.$, com $\left.\alpha \neq \beta\right)$ têm na composição de $\mathcal{J}_{i j}$, tanto para primeiros vizinhos como para interações de mais longa distância. Essa mesma propriedade também foi verificada, por exemplo, para o bulk de $\alpha$ Fe na fase paramagnética [195], e está intimamente relacionada a efeitos não-locais. Vale 
a pena também destacar que as contribuições dos orbitais $d$ para a formação do valor total de $\mathcal{J}_{i j}$ não preserva por completo a simetria $C_{3 h}$ das camadas de $\mathrm{Fe}$ ou de Co, e o acoplamento dos sítios identificados como "3" e "6" apresentam diferentes contribuições principais, advindas de orbitais que não correspondem ao verificado para os demais tipos de sítio na vizinhança. Isso está vinculado, direta ou indiretamente, à existência de dois vetores $\mathbf{D}_{\mathrm{Fe}-\mathrm{Fe}}$ na segunda vizinhança do sistema $\mathrm{Pd} / \mathrm{Fe} / \operatorname{Ir}(111)$ que não seguem a tendência dos demais e direcionam-se paralelamente ao plano da superfície de $\operatorname{Ir}(111)$ (veja a Fig. 5.6(b)), enquanto os demais voltam-se quase na direção perpendicular ao plano (out-of-plane). Para a interação AFM mais expressiva entre pares Fe-Fe ( $3^{\text {os }}$ vizinhos), verifica-se que todas as principais contribuições são negativas, embora se originem de interações mistas para os sítios atômicos "2", "4", "5", e "7", e de interações puras $\left(d_{\alpha}-d_{\alpha}\right)$ nos sítios atômicos "3" e "6".

Tabela 5.7: Principais componentes que constituem os parâmetros de troca em termos das interações entre orbitais $d\left(\mathcal{J}_{i j}^{d_{\alpha}-d_{\beta}}\right)$ para ambos os sistemas investigados: $\mathrm{Pd} / \mathrm{Fe} / \operatorname{Ir}(111)$ e $\mathrm{Pd} / \mathrm{Co} / \operatorname{Ir}(111)$, nas suas respectivas camadas magnéticas. As interações são consideradas entre um átomo típico de cada camada com os sítios definidos na Fig. $\mathbf{5 . 5}$ (para a primeira vizinhança), sendo análogos para a segunda e para a terceira bases de vizinhos. Os números em parêntesis indicam as contribuições efetivas advindas das interações entre os orbitais $d_{\alpha}-d_{\beta}$ correspondentes, em meV.

\begin{tabular}{|c|c|c|c|}
\hline Camada & Vizinhança & Sítios $2 / 4 / 5 / 7$ & Sítios 3/6 \\
\hline \multirow{3}{*}{$\mathrm{Fe}$} & $1^{\text {os }}(\sim 2,72$ & $\begin{array}{c}d_{3 z^{2}-r^{2}}-d_{y z} \\
d_{x^{2}-y^{2}}-d_{y z}(4,87) \\
d_{x z^{-}} d_{x z}(4,18)\end{array}$ & $\begin{array}{c}d_{3 z^{2}-r^{2}}-d_{x y}(9,87) \\
d_{x^{2}-y^{2}}-d_{y z}(4,87) \\
d_{x z^{-}} d_{x z}(2,61)\end{array}$ \\
\hline & $2^{\text {os }}(\sim 4,70 \AA)$ & $\begin{array}{c}d_{x z^{-}} d_{x z}(0,25) \\
d_{3 z^{2}-r^{2}}-d_{3 z^{2}-r^{2}}(0,13) \\
d_{3 z^{2}-r^{2}}-d_{x^{2}-y^{2}}(-0,12)\end{array}$ & $\begin{array}{c}d_{3 z^{2}-r^{2}}-d_{x^{2}-y^{2}}(-0,34) \\
d_{x y^{-}} d_{y z}(-0,29) \\
d_{x y}-d_{x^{2}-y^{2}}(0,27)\end{array}$ \\
\hline & $3^{\text {os }}(\sim 5,43 \AA)$ & $\begin{array}{c}d_{x^{2}-y^{2}}-d_{y z}(-1,04) \\
d_{y z^{-}} d_{x y}(-0,32) \\
d_{x^{2}-y^{2}}-d_{x^{2}-y^{2}}(-0,30)\end{array}$ & $\begin{array}{c}d_{3 z^{2}-r^{2}-} d_{3 z^{2}-r^{2}}(-1,00) \\
d_{x y^{-}} d_{x y}(-0,91) \\
d_{3 z^{2}-r^{2}}-d_{x z}(-0,26)\end{array}$ \\
\hline \multirow{3}{*}{$\mathrm{Co}$} & $1^{\text {os }}(\sim 2,72 \AA)$ & $\begin{array}{ll}d_{x^{2}-y^{2}-} d_{y z} & (2,16) \\
d_{x y^{-}}-d_{x^{2}-y^{2}} & (1,89) \\
d_{3 z^{2}-r^{2}}-d_{y z} & (1,74)\end{array}$ & 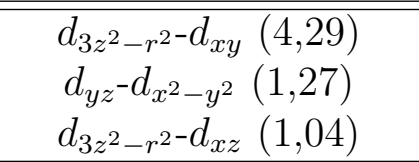 \\
\hline & $2^{\mathrm{os}}(\sim 4,70 \AA)$ & $\begin{array}{cc}d_{x^{2}-y^{2}}-d_{y z} & (0,34) \\
d_{y z}-d_{x^{2}-y^{2}} & (0,31) \\
d_{x y}-d_{y z} & (0,18)\end{array}$ & $\begin{array}{c}d_{x y^{-}} d_{y z}(0,31) \\
d_{3 z^{2}-r^{2}}-d_{x^{2}-y^{2}}(0,21) \\
d_{x z^{-}}-d_{x z}(-0,18)\end{array}$ \\
\hline & $3^{\text {os }}(\sim 5,43 \AA)$ & $\begin{array}{c}d_{x^{2}-y^{2}-d_{y z}}(0,26) \\
d_{x z^{-}} d_{x z}(-0,17) \\
d_{x z}-d_{y z}(0,16)\end{array}$ & $\begin{array}{c}d_{3 z^{2}-r^{2}}-d_{x y}(0,26) \\
d_{x y^{-}} d_{y z}(0,12) \\
d_{x^{2}-y^{2}}-d_{y z}(-0,12)\end{array}$ \\
\hline
\end{tabular}




\subsection{Influência de defeitos no sistema $\mathrm{Pd} / \mathrm{Fe} / \operatorname{Ir}(111)$}

A análise anterior contemplou comparações entre as superfícies de $\mathrm{Pd} / \mathrm{Fe} / \mathrm{Ir}(111)$ e $\mathrm{Pd} / \mathrm{Co} / \mathrm{Ir}(111)$, com o intuito de investigar as origens de uma espiral de spins como estado fundamental da superfície de $\mathrm{Pd} / \mathrm{Fe} / \mathrm{Ir}(111)$ e de uma rede de skyrmions quando um campo magnético perpendicular é aplicado a esta superfície. Entretanto, se desejamos de fato utilizar os skyrmions - em superfícies como o $\mathrm{Pd} / \mathrm{Fe} / \operatorname{Ir}(111)$ - como meios de armazenamento e transporte de informação, faz-se necessário entender como essas estruturas interagem com defeitos que podem eventualmente ocorrer em amostras e materiais reais. Por exemplo, recentemente verificou-se que vórtices magnéticos contendo milhares de átomos de Fe $\left(\sim 10^{4}\right)$ podem ser sucessivamente presos em defeitos de escala atômica quando guiados por campos magnéticos in-plane [196].

Esforços têm sido empregados no sentido de resolver este problema, sobretudo baseados em modelos micromagnéticos e fenomenológicos [197-199]. Contudo, pouco ainda foi explorado na literatura sobre a interação de defeitos com skyrmions via cálculos de primeiros princípios em conjunto com dinâmica atomística de spins. Fernandes et al. [200] analisaram teoricamente o movimento de skyrmions no sistema de $\mathrm{Pd} / \mathrm{Fe} / \mathrm{Ir}(111)$, considerando a inclusão de defeitos $3 d$ ( Cr, Mn, Co) e $4 d(\mathrm{Nb}, \mathrm{Tc}, \mathrm{Ru})$ na camada de Pd. Independentemente do caráter da interação skyrmion-defeito, os autores encontraram um movimento girotrópico, com uma trajetória no sentido horário para as impurezas de pinning e anti-horário para as impurezas de interação repulsiva com os skyrmions. Além disso, Arjana et al. [201], demonstraram a existência de uma vasta gama de tipos de interação skyrmion-defeito, ditadas sobretudo pelo sítio de empilhamento do(s) defeito(s), sua geometria, tamanho, e natureza química.

Visando preencher esta lacuna na literatura e aprofundar o entendimento da interação skyrmion-defeito no espaço-real, investigamos a multicamada $\mathrm{Pd} / \mathrm{Fe} / \mathrm{Ir}(111)$ com um defeito de Ir $(5 d)$ localizado no centro da camada de Fe. Considerou-se, portanto, um cluster contendo 188 átomos não-equivalentes (e calculados de forma autoconsistente), tal qual o apresentado na Fig. 6.13 ${ }^{1}$, imerso em um meio cujos parâmetros de potencial são idênticos aos da superfície pura de $\mathrm{Pd} / \mathrm{Fe} / \mathrm{Ir}(111)$. Obtidos os valores ab-initio dos momentos magnéticos e dos $\mathcal{J}_{i j}$ e $\mathbf{D}_{i j}$ entre todos os pares de átomos $\{i, j\}$ do cluster, iniciou-se então o desafio de descrever o Ir como um defeito isolado no pacote UppASD (veja a Figura 5.20) - desenvolvido para tratar de forma eficiente sistemas com condições periódicas de contorno. Como um dos resultados do presente trabalho, a solução desse

\footnotetext{
${ }^{1}$ No sítio de esfera verde localiza-se a impureza de Ir.
} 
problema envolveu um extenso código em Python3, sendo apresentada com mais detalhes no Apêndice $\mathbf{F}$.

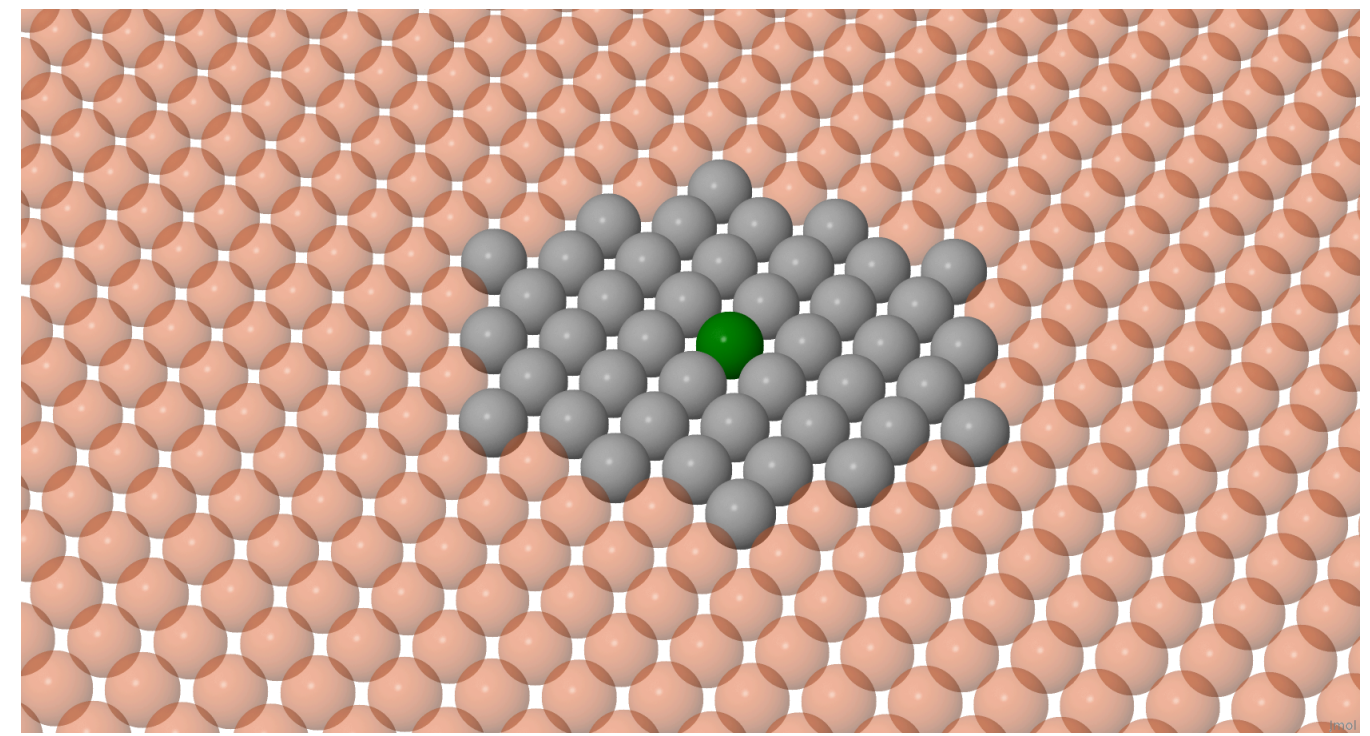

Figura 5.20: Representação esquemática de uma impureza de Ir (esfera verde) embutida na camada de Fe de um substrato perfeito de $\mathrm{Pd} / \mathrm{Fe} / \mathrm{Ir}(111)$, como assumida nos cálculos de dinâmica atomística de spins (via UppASD). As esferas laranjas (translúcidas) representam a região em que os momentos magnéticos, e os parâmetros $\mathcal{J}_{i j}$ e $\mathbf{D}_{i j}$ foram considerados aqueles advindos da superfície pura de $\mathrm{Pd} / \mathrm{Fe} / \operatorname{Ir}(111)$. Já as esferas cinzas (opacas) representam a região não-equivalente considerada no cálculo de primeiros princípios (via RS-LMTO-ASA).

Imagens das simulações de dinâmicas de spin para este caso são mostradas na Fig. 5.21. Nota-se, pela Fig. 5.21(a), que a presença da impureza de Ir induz um estrangulamento local na largura da espiral de spin (estado fundamental do sistema). Quando, por outro lado, aplica-se um campo magnético externo perpendicular à superfície (Fig. $\mathbf{5 . 2 1}(\mathrm{b})$ ), nota-se o surgimento de um skyrmion exatamente na vizinhança do defeito, caracterizando um efeito de pinning. Além disso, em concordância com o reportado na referência [200], a influência da impureza se dá também no perfil do skyrmion, deformandoo. Isso ocorre, essencialmente, pela alteração local dos momentos magnéticos e interações $\mathcal{J}_{i j}$ e $\mathbf{D}_{i j}$ provocadas pela presença do defeito, com parâmetros de Heisenberg Fe-Fe ligeiramente menores e parâmetros de DM Fe-Fe modestamente maiores quando comparados aos mesmos valores obtidos para a multicamada $\mathrm{Pd} / \mathrm{Fe} / \mathrm{Ir}(111)$ limpa. Mais adiante, o estudo do damping nesta mesma configuração trará uma outra perspectiva sobre o efeito de pinning provocado pelo Ir, relacionado ao acoplamento spin-órbita. 


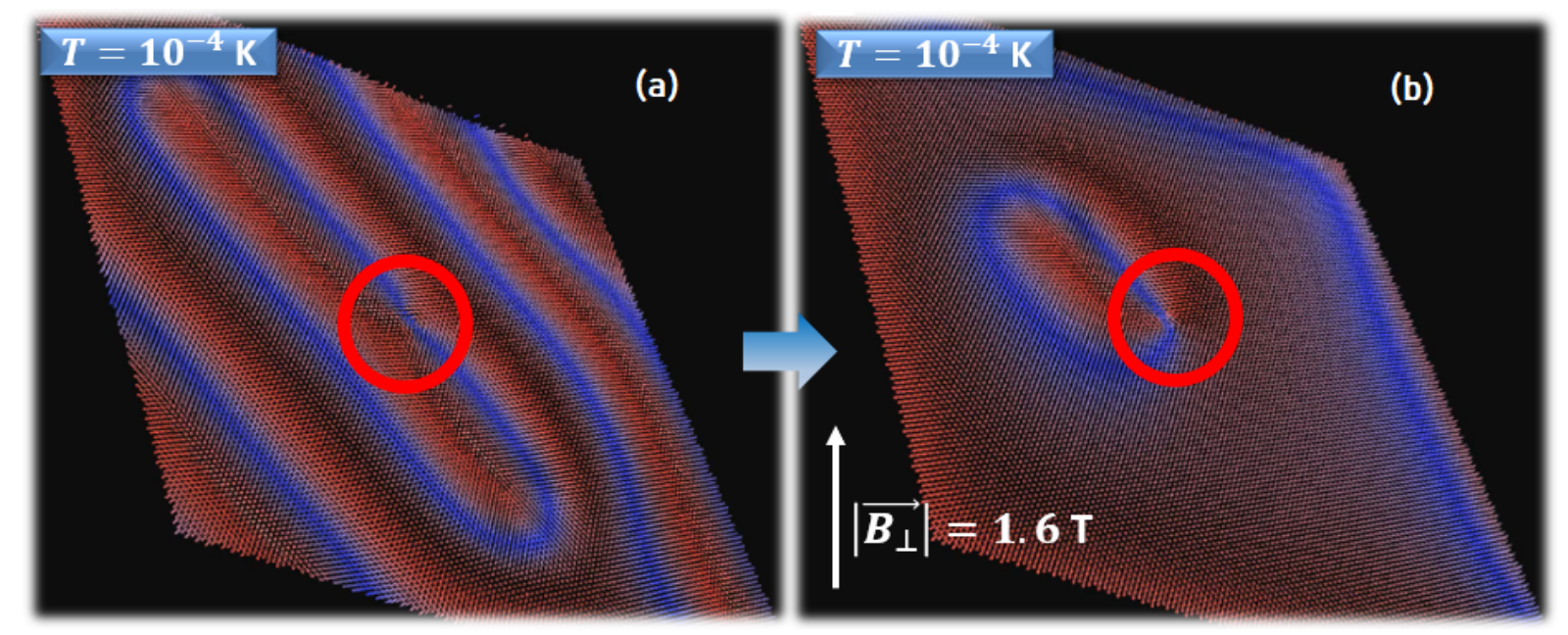

Figura 5.21: Resultados da dinâmica atomística de spins para a multicamada $\mathrm{Pd} / \mathrm{Fe} / \mathrm{Ir}(111)$ com um defeito de Ir na camada de Fe. Situações apresentadas para: (a) campo magnético externo nulo; e $(b)$ campo $B=1,6 \mathrm{~T}$, aplicado perpendicularmente à superfície, ambos à baixas temperaturas $\left(T=10^{-4} \mathrm{~K}\right)$. No centro do círculo vermelho em cada imagem localiza-se o defeito de Ir.

\subsection{Skyrmions em outras multicamadas}

Aproveitando a metodologia desenvolvida no presente trabalho para a investigação das multicamadas $\mathrm{Pd} / \mathrm{Fe} / \mathrm{Ir}(111)$ e $\mathrm{Pd} / \mathrm{Co} / \mathrm{Ir}(111)$, e seus diagramas de fase baseados na dinâmica atomística de spins, estudamos a configuração magnética de outras superfícies puras (i.e., sem a presença de defeitos), a fim de tentar catalogar sistemas ainda não reportados na literatura ou apenas observados experimentalmente. Essa investigação sistemática, portanto, teve como principal objetivo encontrar multicamadas com propriedades interessantes e/ou comuns para o surgimento (ou não) de skyrmions nesses sistemas. Os resultados obtidos estão condensados na Tabela $\mathbf{5 . 8}$.

A partir dos dados exibidos na Tabela 5.8, vale a pena destacar que as multicamadas com estrutura $f c c(111)$ demonstraram uma maior chance de apresentar skyrmions em seu diagrama de fase. Sugere-se, então, que a falta de regras específicas de Moriya [49, 185] para os vetores de DM nas superfícies do tipo $f c c(111)$ esteja diretamente relacionada a este fato. Interessante também notar que o substrato não precisa gerar um forte acoplamento spin-órbita para que se originem skyrmions nas camadas mais externas, tal como no caso da multicamada $\mathrm{Co} / \mathrm{Ni} / \mathrm{Cu}(111)$ (cujo substrato é o $\mathrm{Cu}(111)$ ), embora estes skyrmions tenham maiores dimensões. 
Tabela 5.8: Sistemas multicamadas puros investigados via RS-LMTO-ASA com dinâmica atomística de spins (UppASD), as configurações magnéticas obtidas e a comparação com os resultados teóricos e/ou experimentais disponíveis na literatura.

\begin{tabular}{|c|c|c|c|}
\hline Sistema & Skyrmions & Condições & Observações \\
\hline $\mathrm{Pd} / \mathrm{Fe} / \mathrm{Ir}(111)$ & Sim & $\begin{array}{l}1 \mathrm{~T} \lesssim B \lesssim 3 \mathrm{~T} \mathrm{e} \\
T \lesssim 250 \mathrm{~K}\end{array}$ & $\begin{array}{l}\text { Concorda com a litera- } \\
\text { tura }[52-54] \text { e outras }\end{array}$ \\
\hline $\mathrm{Co} / \mathrm{Ni} / \mathrm{Cu}(111)$ & Sim & $\begin{array}{l}\text { Com } T=10^{-4} \mathrm{~K} \mathrm{e} \\
B=100 \mathrm{mT} \text {, e uma } \\
\text { rede contendo pelo } \\
\text { menos } 1300 \times 1300 \\
\text { spins }\end{array}$ & $\begin{array}{l}\text { Não há literatura para } \\
\text { este sistema ainda }\end{array}$ \\
\hline $\mathrm{Ni} / \mathrm{Ir}_{n} / \mathrm{Pt}(111)$ & Sim & $\begin{array}{l}\text { Para } \mathbf{n}=\mathbf{1}, \quad 0,4 \\
\mathrm{~T} \lesssim B \lesssim 1 \mathrm{~T} \\
\text { (também } \quad \text { ocor- } \\
\text { rendo para } B=0 \\
\mathrm{~T} \text { ) e } T \lesssim 50 \mathrm{~K} ; \\
\text { para } \mathbf{n}=\mathbf{2}, \quad 0 \\
\mathrm{~T} \lesssim B \lesssim 0,4 \mathrm{Te} \\
T \lesssim 40 \mathrm{~K}\end{array}$ & $\begin{array}{l}\text { Não há literatura para } \\
\text { estes sistemas ainda. } \\
\text { Os skyrmions surgem es- } \\
\text { pontaneamente no sistema } \\
\mathrm{Ni} / \mathrm{Ir}_{1} / \mathrm{Pt}(111) \text {, e convivem } \\
\text { em um estado misto com } \\
\text { espirais de spin no estado } \\
\text { fundamental }\end{array}$ \\
\hline $\mathrm{Fe} / \mathrm{Rh} / \mathrm{Ir}(111)$ & Sim & $\begin{array}{l}\operatorname{Em} T=10^{-4} \mathrm{~K}, \mathrm{e} \\
0,2 \lesssim B \lesssim 0,5 \mathrm{~T}\end{array}$ & $\begin{array}{l}\text { Não há literatura para } \\
\text { este sistema ainda. Porém } \\
\text { Romming et al. [202] re- } \\
\text { porta um estado fundamen- } \\
\text { tal de espiral de spin para a } \\
\text { multicamada } \mathrm{Rh} / \mathrm{Fe} / \mathrm{Ir}(111) \\
\text { (stacking fcc), com período } \\
\text { de 1,5 nm, e um estado } \\
\uparrow \uparrow \downarrow \downarrow \text { para o stacking hcp por } \\
\text { causa de interações de spin } \\
\text { de ordem superior (four-spin } \\
\text { [203] ou termo biquadrático) }\end{array}$ \\
\hline
\end{tabular}




\begin{tabular}{|c|c|c|c|}
\hline $\mathrm{Co} / \mathrm{Os}(0001)$ & Talvez & $\begin{array}{l}\text { Talvez seja possível } \\
\text { observar skyrmions } \\
\text { em grandes escalas } \\
\left(\sim 8 \times 10^{5} \text { spins de }\right. \\
\text { largura }) \text {, sugerido } \\
\text { pela relação entre } \\
\text { os stiffness de } J \text { e } \\
D \text { (por enquanto foi } \\
\text { obtido um estado } \\
\text { fundamental FM) }\end{array}$ & $\begin{array}{l}\text { Não há literatura es- } \\
\text { pecífica para este sis- } \\
\text { tema ainda. Belabbes et } \\
\text { al. [204] reporta uma dife- } \\
\text { rença de energia muito pe- } \\
\text { quena } \Delta E=E_{\mathrm{FM}}-E_{\mathrm{AFM}} \approx \\
0,3 \text { meV/átomo entre os es- } \\
\text { tados FM e AFM e uma in- } \\
\text { tensidade de interação DM } \\
\text { não desprezível }\end{array}$ \\
\hline $\mathrm{Pd} / \mathrm{Co} / \mathrm{Ir}(111)$ & Não & - & $\begin{array}{l}\text { Concorda com a referência } \\
{[61]}\end{array}$ \\
\hline $\mathrm{Co} / \mathrm{Ru}(0001)$ & Não & - & $\begin{array}{l}\text { Concorda parcialmente } \\
\text { com a literatura, pois os } \\
\text { skyrmions são metaestáveis } \\
{[109], \text { com } B \sim 150 \mathrm{mT}} \\
\text { (não se observou para esse } \\
\text { campo) - são necessários } \\
\text { testes com escalas maio- } \\
\text { res }\end{array}$ \\
\hline $\mathrm{Fe} / \mathrm{Os}(0001)$ & Não & - & $\begin{array}{l}\text { Concorda com a litera- } \\
\text { tura, formando uma espiral } \\
\text { de spin AFM no estado fun- } \\
\text { damental [205] (veja a Fig. } \\
\mathbf{5 . 2 2} \text { ) }\end{array}$ \\
\hline $\mathrm{Fe} / \mathrm{Pd} / \mathrm{Fe} / \mathrm{Ir}(111)$ & Não & - & $\begin{array}{l}\text { Parcialmente concorda } \\
\text { com a literatura, pois } \\
\text { na Ref. [191] os autores } \\
\text { concluem que o sistema de } \\
\mathrm{Pd} / \mathrm{Fe} / \mathrm{Pd} / \mathrm{Fe} / \mathrm{Ir}(111) \text { apre- } \\
\text { senta uma forte interação } \\
\mathrm{FM} \text {, evitando a formação de } \\
\text { skyrmions }\end{array}$ \\
\hline
\end{tabular}




\begin{tabular}{|c|c|c|c|}
\hline $\mathrm{Co} / \mathrm{Ni} / \mathrm{Cu}(001)$ & Não & - & $\begin{array}{l}\text { Na referência [206], skyrmi- } \\
\text { ons artificiais }{ }^{1} \text { foram criados } \\
\text { incorporando-se um disco in- } \\
\text { plane de } \mathrm{Co} / \mathrm{Ni} \text { em um am- } \\
\text { biente de } \mathrm{Ni} / \mathrm{Cu}(001) \text { out- } \\
\text { of-plane - formando um } \\
\text { caso particular do sistema } \\
\mathrm{Co} / \mathrm{Ni} / \mathrm{Cu}(001)\end{array}$ \\
\hline $\mathrm{Fe} / \mathrm{Ru}(0001)$ & Não & - & $\begin{array}{l}\text { Concorda com a lite- } \\
\text { ratura, pois obtivemos } \\
\text { uma espiral de spin AFM } \\
\text { (próxima a um estado de } \\
\text { Néel) como estado funda- } \\
\text { mental (Fig. } 5.22 \text { ). As } \\
\text { referências [207, 208] repor- } \\
\text { taram uma preferência para } \\
\text { o estado de Néel (com pe- } \\
\text { quena diferença de energia } \\
\text { para a configuração AFM } \\
\text { do tipo row-wise), conside- } \\
\text { rando apenas os parâmetros } \\
\text { de troca }\end{array}$ \\
\hline $\mathrm{Mn} / \operatorname{Ir}(111)$ & Não & - & $\begin{array}{l}\text { Concorda com a litera- } \\
\text { tura. Baseado nas re- } \\
\text { ferências }[204,209] \text {, espera- } \\
\text { se um estado de Néel bas- } \\
\text { tante forte - justamente o } \\
\text { obtido pelos cálculos ab- } \\
\text { initio (Fig. } \mathbf{5 . 2 2} \text { ) }\end{array}$ \\
\hline
\end{tabular}

\footnotetext{
${ }^{1}$ Considera-se um skyrmion artificial aquele que é criado modificando-se as condições naturais de um
} sistema, como a constrição de um disco no material para a formação dessas estruturas. 


\begin{tabular}{|l|l|l|}
\hline Mn/Re(0001) & Não & $\begin{array}{l}\text { Concorda com a litera- } \\
\text { tura. Na referência [210] } \\
\text { recentemente os autores ob- } \\
\text { servaram um estado row- } \\
\text { wise AFM para a superfície } \\
\text { de Mn/Re(0001) (stacking } \\
f c c)\end{array}$ \\
\hline
\end{tabular}

Em alguns sistemas não foi verificada a presença de skyrmions, porém obteve-se configurações magnéticas interessantes, a saber: espirais de spin AFM (Fe/Os(0001) e $\mathrm{Fe} / \mathrm{Ru}(0001))$, row-wise AFM (Mn/Re(0001)), e estados fundamentais de Néel (Mn/Ir(111)).

A Figura 5.22 mostra uma relação verificada entre esses materiais: a evolução da configuração de espiral de spin AFM (com comprimento de onda $\lambda$ cada vez menor) ao estado de Néel se dá, entre outros fatores, com o aumento da magnitude AFM da interação $\mathcal{J}_{i j}$ e da relação $\frac{\mathcal{J}}{|\mathbf{D}|}$ (em uma ordem de grandeza) entre vizinhos magnéticos mais próximos (FeFe ou Mn-Mn). Nestes casos, a aplicação de um campo magnético externo perpendicular não foi capaz de alterar a textura magnética (avaliou-se campos de até $100 \mathrm{~T}$ ). Isso porque, devido à presença de duas sub-redes e a consequente compensação da magnetização, o efeito do campo é cancelado [211].

Assim, o caminho mais comum para a estabilização de skyrmions magnéticos (espiral de spin $\rightarrow$ rede de skyrmions) não é satisfeito nos sistemas AFM analisados. Uma das soluções propostas na literatura para a observação de skyrmions AFM (SkAFM) é a inserção de uma camada magnética de bias [211], que providencia um acoplamento eletrônico adicional entre as camadas, desbalanceando o equilíbrio proporcionado pelas sub-redes (já que este acoplamento ocorre de forma diferente para cada rede). Os sistemas AFM analisados e encontrados na presente tese, portanto, podem ser utilizados em trabalhos futuros neste mesmo arranjo (em conjunto com uma camada de bias) para a formação de SkAFM. Como bem explica a referência [212], a vantagem deste tipo de skyrmion em relação ao FM convencional é o fato de os SkAFM não apresentarem um desvio do movimento por Skyrmion Hall Effect [213], que pode ser um desafio para aplicações que requerem um movimento paralelo dos skyrmions ao longo da direção da corrente aplicada [214]. 


\section{$B=0 ; T=10^{-4} \mathrm{~K}$}

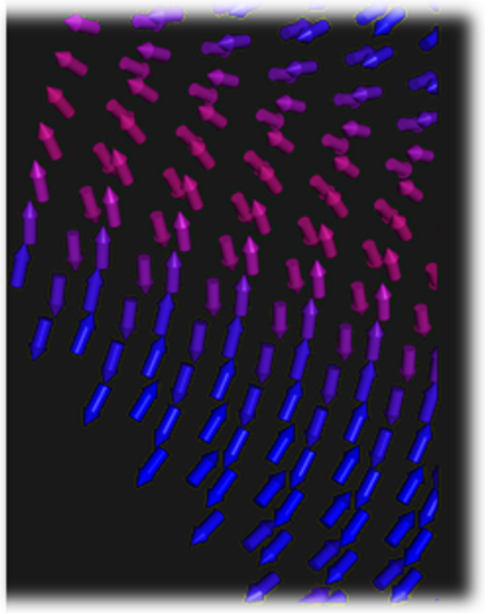

$\mathrm{Fe} / \mathrm{Os}(0001)$

Espiral de spin AFM

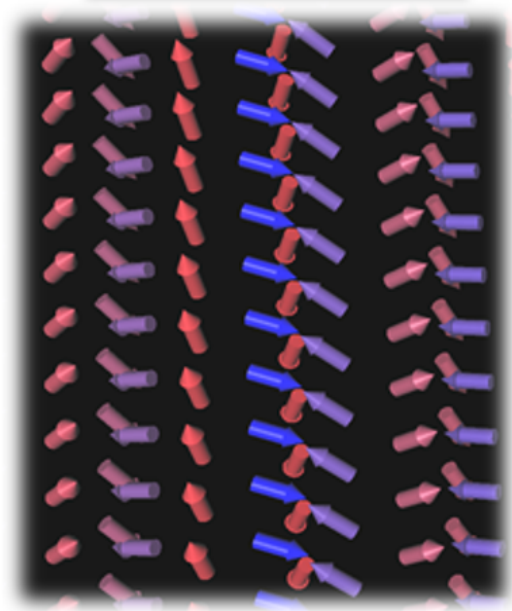

$\mathrm{Fe} / \mathrm{Ru}(0001)$

Espiral de spin AFM

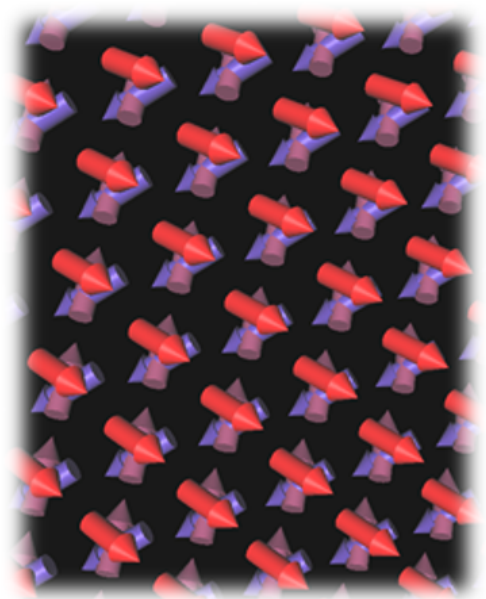

$\mathrm{Mn} / \operatorname{Ir}(\mathbf{1 1 1})$

Estado de Néel

\section{Magnitude crescente de $J_{i j}$ AFM}

Figura 5.22: Resultados da dinâmica atomística de spins para as multicamadas $\mathrm{Fe} / \mathrm{Os}(0001)$ (espiral de spin $\mathrm{AFM}), \mathrm{Fe} / \mathrm{Ru}(0001)$ (espiral de spin $\mathrm{AFM}$ ), e Mn/Ir(111) (estado de Néel). Em todas as situações considerou-se o regime de baixas temperaturas $\left(T=10^{-4} \mathrm{~K}\right)$ e campo magnético externo nulo (embora não haja alteração para $B>0$ $\mathrm{T})$. 


\section{Capítulo 6}

\section{Damping no espaço-real}

\subsection{Formalismo}

O formalismo utilizado para o cálculo do parâmetro de damping no espaço-real, brevemente apresentado neste tópico, é baseado no modelo de torque-correlação (TC) introduzido inicialmente por Kamberský $[215,216]$ e mais tarde elaborado por Gilmore et al. [217]. Esse modelo, por sua vez, pode ser considerado tanto uma generalização como uma extensão do modelo de Breathing Fermi Surface (BFS) [218, 219], proposto em 1976, que relaciona o damping (isto é, a perda de energia e a transferência de momento angular do sistema magnético para o ambiente) com a mudança no formato da superfície de Fermi do material quando a direção do momento magnético é variada. Tal como apontado na literatura, o modelo de torque-correlação é equivalente ao formalismo de Kubo, utilizado, por exemplo, por Ebert et al. [65]. É importante ressaltar que o desenvolvimento teórico apresentado nesta Seção - mais especificamente a passagem do formalismo do espaço-k para o espaço-real, e a dedução de um valor efetivo para o damping em sistemas com mais de uma espécie atômica - é parte dos resultados obtidos no presente trabalho (e está resumido no Material Suplementar da referência [220]).

O ponto inicial é o fato de que a derivada da energia com relação ao momento local $\left(\frac{\partial \epsilon_{\mathbf{k}, n}}{\partial \mathbf{e}}\right.$, quantidade presente no modelo BFS) pode ser calculada utilizando o teorema de Hellmann-Feynman:

$$
\frac{\partial \epsilon_{\mathbf{k}, n}}{\partial \mathbf{e}}=\left\langle\psi_{\mathbf{k}, n}\left|\frac{\partial \mathcal{H}}{\partial \mathbf{e}}\right| \psi_{\mathbf{k}, n}\right\rangle
$$


onde $\mathbf{k}$ é o vetor de onda e $n$ é um índice genérico de banda (estados de Bloch com energia $\left.\epsilon_{\mathbf{k}, n}\right)$. Por causa do acoplamento spin-órbita, a variação na magnetização $\mathbf{m}$ por uma pequena quantidade $\delta \hat{\mathbf{m}}$ gera uma população eletrônica fora de equilíbrio que demora um tempo $\tau$ para relaxar. Esse tempo $\tau$ depende do caminho livre médio dos elétrons. Assim, utilizamos o ângulo $\theta$ para representar a rotação da direção de magnetização $\delta \hat{\mathbf{m}}$. É possível mostrar que a variação de energia devido ao acoplamento spin-órbita é dada por $\epsilon_{\mathbf{k}, n}(\mathbf{e})=\epsilon_{\mathbf{k}, n}(\theta)=\left\langle\psi_{\mathbf{k}, n}\left|e^{i \hat{\mathbf{n}} \cdot \boldsymbol{\sigma} \theta} \mathcal{H}_{s o} e^{-i \hat{\mathbf{n}} \cdot \boldsymbol{\sigma} \theta}\right| \psi_{\mathbf{k}, n}\right\rangle^{1}$, que a derivada com respeito a $\theta$ $\left(\frac{\partial}{\partial \mathbf{e}} \equiv \frac{\partial}{\partial \theta}\right)$ da energia resulta em:

$$
\frac{\partial \epsilon_{\mathbf{k}, n}}{\partial \mathbf{e}}=i\left\langle\psi_{\mathbf{k}, n}\left|e^{i \hat{\mathbf{n}} \cdot \boldsymbol{\sigma} \theta}\left[\hat{\mathbf{n}} \cdot \boldsymbol{\sigma}, \mathcal{H}_{s o}\right] e^{-i \hat{\mathbf{n}} \cdot \boldsymbol{\sigma} \theta}\right| \psi_{\mathbf{k}, n}\right\rangle,
$$

onde $\boldsymbol{\sigma}$ é o vetor de matrizes de Pauli $2 \times 2\left(\boldsymbol{\sigma}=\left(\sigma^{x}, \sigma^{y}, \sigma^{z}\right)\right)$, $\hat{\mathbf{n}}$ a direção ao redor da qual o momento local foi rodado, e $\mathcal{H}_{s o}$ a Hamiltoniana de spin-órbita. Avaliando a derivada da Eq. 6.2 no ponto de equilíbrio $(\theta=0)$, fica-se $\operatorname{com} \frac{\partial \epsilon_{\mathbf{k}, n}}{\partial \mathbf{e}}=i\left\langle\psi_{\mathbf{k}, n}\left|\left[\hat{\mathbf{n}} \cdot \boldsymbol{\sigma}, \mathcal{H}_{s o}\right]\right| \psi_{\mathbf{k}, n}\right\rangle$. No regime colinear, em que todos os spins estão alinhados na direção $z$, denominamos $\hat{T}^{\mu}=$ $\left[\sigma^{\mu}, \mathcal{H}_{s o}\right]$ o operador de torque para uma rotação em torno da direção $\mu=\{x, y, z\}$. Com esse resultado, e inserindo a expressão de $\frac{\partial \epsilon_{\mathbf{k}, n}}{\partial \mathbf{e}}$ no modelo BFS, no qual se define um tensor de damping (adotando-se a aproximação isotrópica de tempo de relaxação, i.e. $\tau_{\mathbf{k}, n} \rightarrow \tau$ ) dado por

$$
\alpha^{\nu \mu}=\frac{g \pi}{m_{t}} \sum_{n, m} \frac{d \mathbf{k}}{(2 \pi)^{3}} \eta\left(\epsilon_{\mathbf{k}, n}\right)\left(\frac{\partial \epsilon_{\mathbf{k}, n}}{\partial \theta}\right)_{\nu}\left(\frac{\partial \epsilon_{\mathbf{k}, m}}{\partial \theta}\right)_{\mu} \frac{\tau}{\hbar}
$$

onde $g$ é o fator de Landé, $m_{t}$ é o momento total $\left(m_{t}=m_{S}+m_{L}\right), \nu, \mu=\{x, y, z\}$ são índices de coordenadas cartesianas, $\eta\left(\epsilon_{\mathbf{k}, n}\right)=\left.\frac{\partial f(\epsilon)}{\partial \epsilon}\right|_{\epsilon_{\mathbf{k}, n}}$ é a derivada com respeito à energia da função de distribuição de Fermi, $f(\epsilon)$, avaliada em $\epsilon_{\mathbf{k}, n}$, e $m, n$ são índices de banda, pode-se escrever a expressão final para o parâmetro de damping $\alpha^{\nu \mu}$ no espaço recíproco como:

$$
\alpha^{\nu \mu}=\frac{g \pi}{m_{t}} \sum_{n, m} \int \Gamma_{n m}^{\nu}(k) \Gamma_{n m}^{\mu}(k) W_{n m}(\mathbf{k}) \frac{d \mathbf{k}}{(2 \pi)^{3}},
$$

na qual $\Gamma_{n m}^{\mu}(k) \equiv\left\langle\psi_{\mathbf{k}, n}\left|\hat{T}^{\mu}\right| \psi_{\mathbf{k}, m}\right\rangle$, e $W_{n m}(\mathbf{k})$ é um fator de peso nos eventos de espalhamento que depende da derivada da distribuição de Fermi-Dirac $f(\epsilon)$ com respeito à energia, e autoestados da equação de Kohn-Sham, representados por uma forma ligeira-

\footnotetext{
${ }^{1}$ Rotação dos estados dentro da esfera de Bloch pela aplicação do operador de rotação $e^{i \hat{\mathbf{n}} \cdot \boldsymbol{\sigma} \theta}$.
} 
mente mais geral de uma função espectral, $A(\epsilon)^{1}$. Explicitamente, escreve-se o fator de peso $W_{n m}$ como $W_{n m}(\mathbf{k})=\int d \epsilon \eta(\epsilon) A_{\Lambda}^{\mathbf{k}, n}(\epsilon) A_{\Lambda}^{\mathbf{k}, m}(\epsilon)$. Assim, sendo a largura das funções espectrais $A_{\Lambda}^{\mathbf{k}, n}(\epsilon)$ e $A_{\Lambda}^{\mathbf{k}, m}(\epsilon)$ determinado pelo parâmetro $\Lambda=\frac{h}{\tau}$ (sendo $h$ a constante de Planck) $)^{2}$, e sabendo que $W_{n m}$ (e, portanto, $\alpha^{\mu \nu}$ ) é não-nulo para $n \neq m$ apenas quando existe uma interseção entre as distribuições Lorentzianas, então o damping de Gilbert depende diretamente desse parâmetro $\Lambda$ (e pode ter contribuições não-locais, desde que exista uma região de interseção entre as funções espectrais). Portanto, é possível definir dois tipos de contribuições para o damping em um determinado sítio $m$ do material: $(i)$ uma contribuição onsite, no qual basta tomar $n=m$ na Eq. 6.4; e (ii) uma contribuição $n \tilde{a}$ o-local, quando $n \neq m$. Isso nos induz a definir um valor total para o damping em um determinado sítio $m$ do material, $\alpha_{m}^{\text {tot }}$ (que deve existir para cada elemento $\nu \mu$ do tensor, aqui suprimida a notação), calculado pelo somatório de todos os valores onsite e não-locais nesse sítio, a saber:

$$
\alpha_{m}^{t o t}=\sum_{n} \alpha_{n m}
$$

definido para cada elemento $\nu \mu$ do tensor de damping, $\boldsymbol{\alpha}$.

Usando a representação de Lehmann para as funções de Green $\hat{G}^{ \pm} \operatorname{retardadas}\left(\hat{G}^{+}\right)$ ou avançadas $\left(\hat{G}^{-}\right)$, reescreve-se a função espectral como [67]

$$
\operatorname{Im} \hat{G}^{ \pm}(\mathbf{k}, \epsilon \pm i \Lambda)=\mp \sum_{n}\left|\psi_{\mathbf{k}, n}\right\rangle\left\langle\psi_{\mathbf{k}, n}\right| A_{\Lambda}\left(\epsilon-\epsilon_{\mathbf{k}, n}\right),
$$

e a Eq. 6.4 então fica:

$$
\alpha^{\nu \mu}=\frac{g}{m_{t} \pi} \iint \eta(\epsilon) \operatorname{Tr}\left(\hat{T}^{\nu} \operatorname{Im} \hat{G}^{+} \hat{T}^{\mu} \operatorname{Im} \hat{G}^{+}\right) \frac{d \mathbf{k}}{(2 \pi)^{3}} d \epsilon
$$

já bastante semelhante ao formalismo de Kubo para o cálculo do mesmo parâmetro (veja, por exemplo, a referência [65]). Nesse caso, a forma explícita para a $A_{\Lambda} \equiv A_{\Lambda}^{\mathbf{k}, n} \equiv$

\footnotetext{
${ }^{1}$ Com a derivação da forma final de $\alpha^{\mu \nu}$ (Eq. 6.4), a função espectral $A(\epsilon)$ tem a forma de uma distribuição Lorentziana centrada na energia $\epsilon$.

${ }^{2}$ Por esse motivo, refere-se a esse termo como o broadening $\Lambda$ da função espectral.
} 
$A_{\Lambda}\left(\epsilon-\epsilon_{\mathbf{k}, n}\right)$ será uma função Lorentziana do tipo:

$$
A_{\Lambda}\left(\epsilon-\epsilon_{\mathbf{k}, n}\right)=\frac{\Lambda}{\left(\epsilon-\epsilon_{\mathbf{k}, n}\right)^{2}+\Lambda^{2}}
$$

cuja integral em todo o espaço $\int_{-\infty}^{\infty} A_{\Lambda}(\epsilon) d \epsilon=\pi$, independentemente do valor de $\Lambda$. Essa propriedade torna possível derivar a expressão 6.7 para os elementos do tensor de damping. Além disso, a partir da Eq. 6.8, tem-se uma expressão explícita para as funções $A_{\Lambda}$ que compõem o fator de peso $W_{n m}(\mathbf{k})$ no espaço-recíproco. A Figura 6.1, abaixo, mostra as transições intrabandas (que dão origem ao damping onsite) e as transições interbandas (que dão origem ao damping não-local) como dependentes do overlap das funções Lorentzianas. Quanto maior for a região de overlap (proporcional à largura $\Lambda$, sobretudo no caso do damping onsite), maior será o fator de peso $W_{n m}$, e, portanto, o valor da componente de damping $\alpha^{\nu \mu}$ (Eq. 6.4). A largura $\Lambda$ das funções Lorentzianas, por sua vez, é proporcional ao acoplamento elétron-fônon no material [165].

Transições interbanda

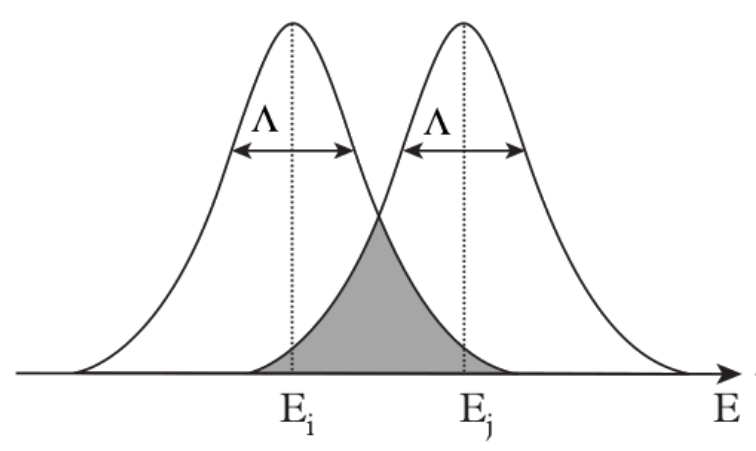

Transições intrabanda

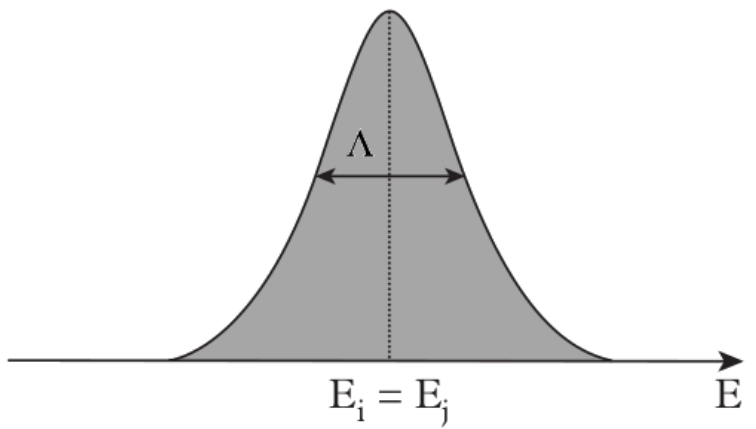

Figura 6.1: Transições interbanda (que dão origem ao damping não-local) e intrabanda (que dão origem ao damping onsite) no modelo de torque-correlação. Aqui, $\Lambda$ representa a largura das funções Lorentzianas $A_{\Lambda}$ centradas nas energias $\epsilon_{\mathbf{k}, n}$ (Eq. 6.8).

As transições intrabanda estão em atual discussão na literatura. No modelo de Kamberskỳ, Costa e Muniz [221] demonstraram que, no caso de sistemas perfeitos (e particularmente em ambientes com elevado acoplamento spin-órbita), o valor de $\alpha \rightarrow \infty$ devido ao espalhamento intrabanda. No mesmo período, Ma et al. [222] concluíram, a partir de experimentos em filmes de FePt, que não há indicação da existência de um termo intrabanda em $\alpha$. Logo em seguida, Edwards [223] demonstrou que é possível reduzir o formalismo proposto por Costa e Muniz ao de Kamberskỳ, mas com o termo de espalhamento intrabanda (de ordem $\xi^{3}$ ) ausente, resolvendo a inconsistência existente entre os dois métodos. Entretanto, recentemente os experimentos Khodadadi et al. [224] mos- 
traram que o espalhamento intrabanda no Fe epitaxial é um mecanismo importante para o damping, apresentando um excelente acordo com os cálculos de Gilmore et al. [217] e Mankovsky et al. [66], ambos baseados no modelo de Kamberskỳ.

A expressão 6.7 ainda é válida para o espaço-recíproco. No entanto, a tradução para o espaço-real é direta, e o parâmetro de damping $\alpha_{i j}^{\mu \nu}$ entre os sítios $i$ e $j$ do material pode ser definido como $(\mathrm{a} T=0 \mathrm{~K})^{1}$ :

$$
\alpha_{i j}^{\mu \nu}=\frac{g}{\pi m_{t}} \int_{-\infty}^{+\infty} \eta(\epsilon) \operatorname{Tr}\left(\hat{T}_{i}^{\mu} \operatorname{Im} \hat{G}_{i j}^{+} \hat{T}_{j}^{\nu} \operatorname{Im} \hat{G}_{j i}^{+}\right) d \epsilon
$$

na qual $\epsilon_{F}$ é a energia de Fermi do sistema, e $\operatorname{Im} \hat{G}_{i j}^{+} \equiv \operatorname{Im} \hat{G}_{i j}^{+}(\epsilon)$ é a parte imaginária da função de Green retardada (não-local) entre os sítios $i$ e $j$ calculada pelo método RS-LMTO-ASA [134]. De um ponto de vista prático, considera-se a própria função $\hat{G}_{i j}^{+}$ obtida pela autoconsistência para o cálculo da componente $\alpha_{i j}^{\mu \nu}$ tal como na Eq. 6.9. Note que, embora a propriedade para função Lorentziana $A_{\Lambda}$ seja de integração em todo o espaço, a Eq. 6.9 leva em conta a integração apenas até $\epsilon_{F}$, pois estamos tratando o sistema no estado fundamental ${ }^{2}$. Levando em conta a forma explícita da Hamiltoniana de spin-órbita $\mathcal{H}_{\text {so }}$ (veja a referência [118], por exemplo), os operadores de torque nas direções $x, y, z$ globais $\left(\hat{T}^{x}, \hat{T}^{y}, \hat{T}^{z}\right)$ são escritos em termos dos operadores de momento angular $\hat{L}_{x}, \hat{L}_{y}, \hat{L}_{z}$ da seguinte maneira:

$$
\begin{array}{r}
\hat{T}^{x}=\left(\begin{array}{cc}
2 i \hat{L}_{y} & -2 \hat{L}_{z} \\
2 \hat{L}_{z} & -2 i \hat{L}_{y}
\end{array}\right) \\
\hat{T}^{y}=\left(\begin{array}{cc}
-2 i \hat{L}_{x} & 2 i \hat{L}_{z} \\
2 i \hat{L}_{z} & 2 i \hat{L}_{x}
\end{array}\right) \\
\hat{T}^{z}=\left(\begin{array}{cc}
0 & 2 \hat{L}_{x}-2 i \hat{L}_{y} \\
-2 \hat{L}_{x}-2 i \hat{L}_{y} & 0
\end{array}\right),
\end{array}
$$

sendo todas matrizes $2 \times 2$ no espaço de spins, mas matrizes $18 \times 18$ no espaço de orbitais, considerando a base de 9 orbitais ( $1 s, 3 p$, e $5 d$ ) adotada pelo LMTO-ASA [125]. Assim, o produto $\hat{T}_{i}^{\mu} \operatorname{Im} \hat{G}_{i j}^{+} \hat{T}_{j}^{\nu} \operatorname{Im} \hat{G}_{j i}^{+}$da Eq. 6.9 resulta em uma matriz $18 \times 18$ dependente da energia $\epsilon$ (para cada sítio não-equivalente do problema).

\footnotetext{
${ }^{1}$ Apesar de a integração ser de $-\infty$ a $+\infty$, o integrando só não será nulo nas regiões de "atuação" das funções de Green, em geral próximas do nível de Fermi $\left(\epsilon_{F}\right)$ do material.

${ }^{2}$ Lembre-se que as funções de Green são calculadas a partir da estrutura eletrônica convergida pelo processo autoconsistente da DFT.
} 
É importante destacar que, no método RS-LMTO-ASA, a função de Green obtida com auxílio do Método de Recorrência de Haydock [135], em conjunto com o terminador de Beer-Pettifor [136], é naturalmente uma função retardada do tipo $\hat{G}^{+}\left(\epsilon+i \Lambda_{R S}\right)$, na qual o broadening $\Lambda_{R S}$ relaciona-se com a parte imaginária do terminador ${ }^{1}-\mathrm{e}$, portanto, é particular de cada sistema investigado. Uma sugestão para a obtenção desse valor diretamente será discutida mais adiante.

Após o cálculo de cada componente $\alpha_{i j}^{\mu \nu}$ no caso colinear, tem-se um tensor de damping $\boldsymbol{\alpha}_{i j}$ do tipo:

$$
\boldsymbol{\alpha}_{i j}=\left(\begin{array}{ccc}
\alpha^{x x} & \alpha^{x y} & \alpha^{x z} \\
\alpha^{y x} & \alpha^{y y} & \alpha^{y z} \\
\alpha^{z x} & \alpha^{z y} & \alpha^{z z}
\end{array}\right)
$$

que é parte da equação atomística de LLG para o $i$-ésimo sítio equivanete à Eq. 4.1, porém considerando agora o damping como sendo um tensor [225]:

$$
\frac{\partial \mathbf{m}_{i}}{\partial t}=\mathbf{m}_{i} \times\left(-\gamma \mathbf{B}_{i}^{\mathrm{eff}}+\sum_{j} \frac{\boldsymbol{\alpha}_{i j}}{m_{j}} \cdot \frac{\partial \mathbf{m}_{j}}{\partial t}\right) .
$$

Recentemente, Mankovsky et al. [226] mostraram como calcular o damping para o caso de uma configuração não-colinear de spins, e que sugerimos que possa ser reproduzida no formalismo apresentado aqui apenas modificando os operadores de torque, ao considerar que o pólo $(\theta=0)$ de rotação está localizado em cada direção de $\operatorname{spin} \mathbf{s}^{i}=\left(s_{1}^{i}, s_{2}^{i}, s_{3}^{i}\right)$ local do sítio $i$, ao invés da direção $z$ global. Os dois demais eixos de rotação locais são projeções das direções $x$ e $y$ nesse novo sistema de coordenadas (podem ser obtidos, por exemplo, por uma rotação do sistema $x y z$ usual em torno do mesmo eixo que provocou a variação o eixo- $z$ para $\mathbf{s}^{i}$ ).

Por fim, para fins de análises qualitativas e comparação com quantidades que podem ser medidas experimentalmente, pode-se também fazer uso da fórmula simplificada de

\footnotetext{
${ }^{1}$ No formalismo de Beer-Pettifor, o terminador é definido como $t(E)=$ $\frac{1}{2}[(E-a) \pm \sqrt{(E-a-2 b)(E-a+2 b)}]$, onde $a$ e $b$ são os coeficientes da fração continuada após $L L$ níveis de recursão. O fato de $t(E)$ ser calculado por uma raiz quadrada que sempre apresenta um termo negativo gera um resultado imaginário.
} 
Kamberský [215, 218, 227]:

$$
\alpha=\frac{1}{\gamma m_{S}}\left(\frac{\gamma}{2}\right)^{2} n\left(E_{F}\right) \xi^{2} \frac{(g-2)^{2}}{\tau_{e}}
$$

em que $\gamma$ é a razão giromagnética, $\tau_{e}$ é o tempo de espalhamento eletrônico, $n\left(E_{F}\right)$ representa a LDOS no nível de Fermi, $\xi$ é o strength do acoplamento spin-órbita, $m_{S}$ é o momento magnético de spin, e $g$ é o fator espectroscópico [228, 229]. Note que a Eq. 6.13 estabelece uma relação direta entre $\alpha$ e $n\left(E_{F}\right)$, geralmente discutida na literatura [167].

\subsubsection{Damping de Gilbert para materiais com diferentes tipos atômicos}

Em experimentos de ressonância magnética (FMR), os momentos magnéticos são excitados em um modo coletivo, denominado modo de Kittel, no qual todos os spins da amostra precessionam em fase com a mesma amplitude. Neste cenário, faz sentido definir um macrospin ( $\mathbf{M}^{\mathrm{eff}}$ ) como a soma de todos os momentos magnéticos locais (individuais), ou seja:

$$
\mathbf{M}^{\mathrm{eff}}=\sum_{i} \mathbf{m}_{i}
$$

Assim, tomando como base a Eq. 6.12, a dinâmica dessa magnetização coletiva pode também ser bem descrita pela equação de Landau-Lifshitz-Gilbert, a saber:

$$
\frac{\partial \mathbf{M}^{\mathrm{eff}}}{\partial t}=\mathbf{M}^{\mathrm{eff}} \times\left(-\gamma \mathbf{B}^{\mathrm{eff}}+\frac{\boldsymbol{\alpha}^{\mathrm{eff}}}{M^{\mathrm{eff}}} \cdot \frac{\partial \mathbf{M}^{\mathrm{eff}}}{\partial t}\right)
$$

na qual $M^{\mathrm{eff}}=\left|\mathbf{M}^{\mathrm{eff}}\right|=\left|\sum_{i} \mathbf{m}_{i}\right| \leq \sum_{i}\left|\mathbf{m}_{i}\right|$. Como aqui, em princípio, queremos descrever um estado colinear, pode-se demonstrar que apenas um campo magnético externo, bem como a anisotropia magnética do sistema, podem influenciar na precessão dos momentos. Assumindo que a anisotropia é a mesma para todos os sítios ${ }^{1}$ ou pequena demais quando comparada ao campo magnético externo, considera-se $\mathbf{B}_{i}=\mathbf{B}$ como isotrópico (isto é, independente do sítio). Além disso, assume-se o damping como um valor único associado

\footnotetext{
${ }^{1} \mathrm{O}$ que não é sempre necessariamente verdade.
} 
a cada sítio (ou seja, $\boldsymbol{\alpha}=\alpha$ ).

Nesse contexto, tomando-se a derivada temporal da Eq. $\mathbf{6 . 1 4}\left(\frac{\partial \mathbf{M}^{\mathrm{eff}}}{\partial t}=\sum_{i} \frac{\partial \mathbf{m}_{i}}{\partial t}\right)$, e aplicando-a na Eq. 6.15, obtém-se o seguinte:

$$
\begin{aligned}
& \frac{\partial \mathbf{M}^{\mathrm{eff}}}{\partial t}=\sum_{i} \frac{\partial \mathbf{m}_{i}}{\partial t}= \sum_{i}\left[\mathbf{m}_{i} \times\left(-\gamma \mathbf{B}_{i}+\frac{\alpha_{i}}{\left|\mathbf{m}_{i}\right|} \frac{\partial \mathbf{m}_{i}}{\partial t}\right)\right] \\
&=-\gamma\left[\sum_{i} \mathbf{m}_{i}\right] \times \mathbf{B}+\left[\sum_{i} \mathbf{m}_{i} \times \frac{\alpha_{i}}{\left|\mathbf{m}_{i}\right|} \frac{\partial \mathbf{m}_{i}}{\partial t}\right] \\
&=-\gamma \mathbf{M}^{\mathrm{eff}} \times \mathbf{B}+\left[\sum_{i} \mathbf{m}_{i} \times \frac{\alpha_{i}}{\left|\mathbf{m}_{i}\right|} \frac{\partial \mathbf{m}_{i}}{\partial t}\right]
\end{aligned}
$$

Comparando o resultado da Eq. 6.16 com o descrito na Eq. 6.15 (e considerando $\left.\boldsymbol{\alpha}^{\mathrm{eff}}=\alpha^{\mathrm{eff}}\right)$ :

$$
\mathbf{M}^{\mathrm{eff}} \times\left(\frac{\alpha^{\mathrm{eff}}}{M^{\mathrm{eff}}} \frac{\partial \mathbf{M}^{\mathrm{eff}}}{\partial t}\right)=\left[\sum_{i} \mathbf{m}_{i} \times \frac{\alpha_{i}}{\left|\mathbf{m}_{i}\right|} \frac{\partial \mathbf{m}_{i}}{\partial t}\right]
$$

a partir da qual pode-se escrever que $\mathbf{M}^{\mathrm{eff}}=M^{\mathrm{eff}} \mathbf{e}^{\mathrm{eff}}$, e $\mathbf{m}_{i}=m_{i} \mathbf{e}_{i}\left(\mathbf{e}^{\mathrm{eff}}\right.$ e $\mathbf{e}_{i}$ simbolizam, respectivamente, a direção dos momentos do macrospin e no sítio atômico $i$ ). Como se trata de um movimento coerente de spins, naturalmente $\frac{\partial \mathbf{e}_{i}}{\partial t}=\frac{\partial \mathbf{e}^{\text {eff }}}{\partial t}$ e $\mathbf{e}_{i}=\mathbf{e}^{\text {eff }}$, e, portanto:

$$
\mathbf{e}^{\mathrm{eff}} \times\left(M^{\mathrm{eff}} \alpha^{\mathrm{eff}}\right) \frac{\partial \mathbf{e}^{\mathrm{eff}}}{\partial t}=\left[\sum_{i} \mathbf{e}_{i} \times m_{i} \alpha_{i} \frac{\partial \mathbf{e}_{i}}{\partial t}\right]=\mathbf{e}^{\mathrm{eff}} \times\left(\sum_{i} m_{i} \alpha_{i}\right) \frac{\partial \mathbf{e}^{\mathrm{eff}}}{\partial t} .
$$

Logo, no caso de sistemas com mais de um tipo atômico em que se deseja calcular o damping efetivo $\left(\alpha^{\mathrm{eff}}\right)$ de um conjunto local de átomos, ou mesmo de todos os sítios que constituem o material (ou de uma superfície, por exemplo), o valor é calculado como uma média de todos os dampings, ponderada pelo momento magnético local de cada sítio $\left(m_{i}\right)$, 
ou seja $a^{1}$ :

$$
\alpha^{\mathrm{eff}}=\frac{1}{M^{\mathrm{eff}}} \sum_{i} m_{i} \alpha_{i}^{t o t}=\frac{1}{\sum_{i} m_{i}} \sum_{i} m_{i} \alpha_{i}^{t o t}
$$

Assim, para todos os casos em que foi necessário considerar mais de um tipo atômico, utilizou-se a equação acima para o cálculo do damping efetivo. Entretanto, como o resultado está, da mesma forma que o damping total, relacionado a um único sítio (médio), referiremos $\alpha^{\text {eff }}$ como $\bar{\alpha}_{t o t}$ daqui em diante - a fim de deixar a notação consistente.

\subsection{Primeiros testes em sistemas bulk}

O formalismo apresentado na seção anterior foi implementado no pacote RS-LMTOASA usando a linguagem de programação FORTRAN90. Os arquivos do código-fonte modificados para esse fim foram o buildham.f90 e o bgreen_jij.F90. Utiliza-se como arquivo de entrada o damping.in, que será descrito em mais detalhes no Apêndice $\mathbf{D}$.

\subsubsection{Bulk de Ni fcc}

Iniciamos nossos testes com o bulk de $\mathrm{Ni}$ de estrutura cúbica de face centrada $(f c c)$, modelado do espaço-real por um cluster contendo $\sim 66.427$ átomos (substrato puro), com o parâmetro de rede experimental ( $a=3,52 \AA[188])$. A fim de analisar as contribuições onsite e não-locais para o damping em um sítio típico nessa estrutura, estendemos a somatória apresentada na Eq. 6.5 para um pouco mais de 3 parâmetros de rede, resultando em um total de 459 pares $\mathrm{Ni}-\mathrm{Ni}$, distribuídos segundo a simetria da rede $f c c$, a partir de uma posição central. A Figura 6.2, a seguir, representa o cluster de átomos de $\mathrm{Ni}$ formado para o cálculo do damping total. Para este caso, utilizamos o parâmetro de corte $L L=31$ para o terminador de Beer-Pettifor. Os parâmetros $L L$ e o tamanho do cluster no espaço-real foram inicialmente escolhidos, pois são os que conhecidamente reproduzem bem as propriedades magnéticas e eletrônicas do Ni.

\footnotetext{
${ }^{1}$ Essa expressão só é possível supondo que o sistema é composto por um coletivo e coerente de spins colineares, que precessionam todos com a mesma velocidade. Para sistemas em que estas condições não são válidas, ainda há a lacuna de um desenvolvimento matemático para tal.
} 


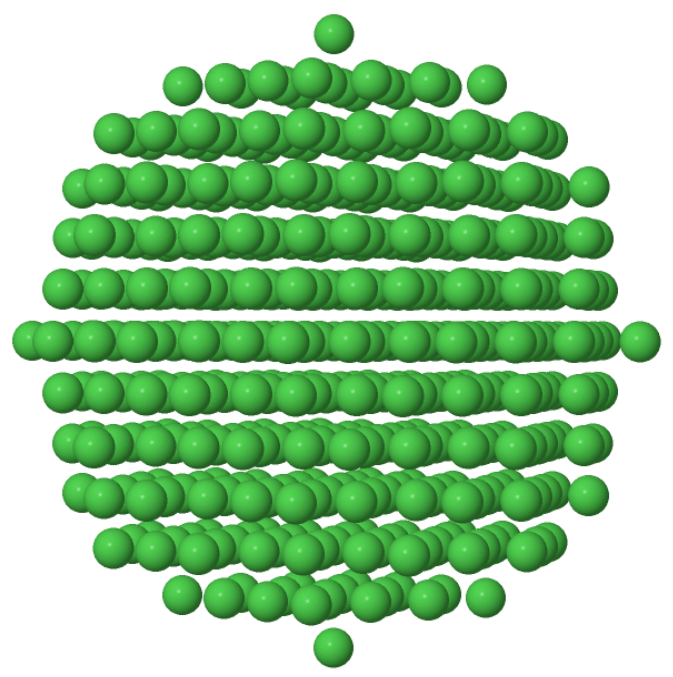

Figura 6.2: Representação esquemática do cluster de Ni no espaço-real, com estrutura fcc e contendo 459 átomos, utilizado no cálculo das contribuições onsite e não-locais do parâmetro de damping de Gilbert.

No caso de uma configuração magnética colinear, o tensor de damping onsite $\boldsymbol{\alpha}_{i i}$ (sem dimensão) obtido para um sítio típico no Ni bulk:

$$
\boldsymbol{\alpha}_{i i_{\mathrm{Ni}}}=\left(\begin{array}{rrr}
0,048026 & 0,000000 & 0,000000 \\
0,000000 & 0,048026 & 0,000000 \\
0,000000 & 0,000000 & 0,016542
\end{array}\right)
$$

É importante notar que, devido à simetria cúbica do sistema, esperava-se exatamente valores diferentes de zero apenas nos componentes $\alpha^{x x}, \alpha^{y y}\left(\operatorname{com} \alpha^{x x}=\alpha^{y y}\right)$, e $\alpha^{z z}$. Além disso, como o valor de $\alpha^{z z}$ advém, de acordo com a definição dos operadores de torque, de uma rotação do estado de partícula única do pólo (em z) em torno dele mesmo, não sabemos ainda exatamente seu significado físico (está em discussão). Observando a Eq. 6.12, a matriz diagonal encontrada para $\boldsymbol{\alpha}_{i i_{\mathrm{Ni}}}$ sugere que há equações desacopladas para a dinâmica do momento $\mathbf{m}_{i}$ em cada uma das três direções espaciais $(x, y, z)$, o que nos incentiva a definir uma constante de damping $\alpha$ como a média das duas componentes $\alpha^{x x}$ e $\alpha^{y y}$, a saber: $\alpha=\frac{1}{2}\left(\alpha^{x x}+\alpha^{y y}\right)$. Essa definição pode ser estendida também para as contribuições não-locais. Logo, para o bulk de $\mathrm{Ni}$, as constantes de damping total $\left(\alpha_{\text {tot }}\right)$ e onsite $\left(\alpha_{i i}\right)$ obtidas, além de uma comparação com a literatura são apresentados na Tabela 6.1, abaixo. 
Tabela 6.1: Contribuição onsite e damping total obtidos para um sítio típico no bulk de Ni fcc, e comparação com os resultados da literatura.

\begin{tabular}{|l|c|c|}
\hline & Este trabalho & Literatura \\
\hline Onsite $\left(\alpha_{i i}\right)$ & 0,0577 & $0,0216[225]$ \\
\hline Total $\left(\alpha_{t o t}\right)$ & 0,0278 & $0,018[217] / 0,0237[230]$ \\
\hline
\end{tabular}

Em adição ao bom acordo entre os resultados obtidos via RS-LMTO-ASA e os apresentados na literatura (sobretudo para $\alpha_{t o t}$ ), observa-se que a contribuição não-local deve ter uma influência global negativa na composição do parâmetro de damping total, já que $\alpha_{i i}>\alpha_{\text {tot }}$. Portanto, a fim de analisar como essas contribuições não-locais influenciam na composição de $\alpha_{t o t}$, uma alternativa é investigar o damping não-local $\alpha_{i j}(i \neq j)$ como função da distância de um sítio típico no bulk de Ni. Essa relação é apresentada pelo gráfico da Figura 6.3, em termos da distância $d$ normalizada pelo parâmetro de rede $a$ do material $(d \leq 3 a)$.

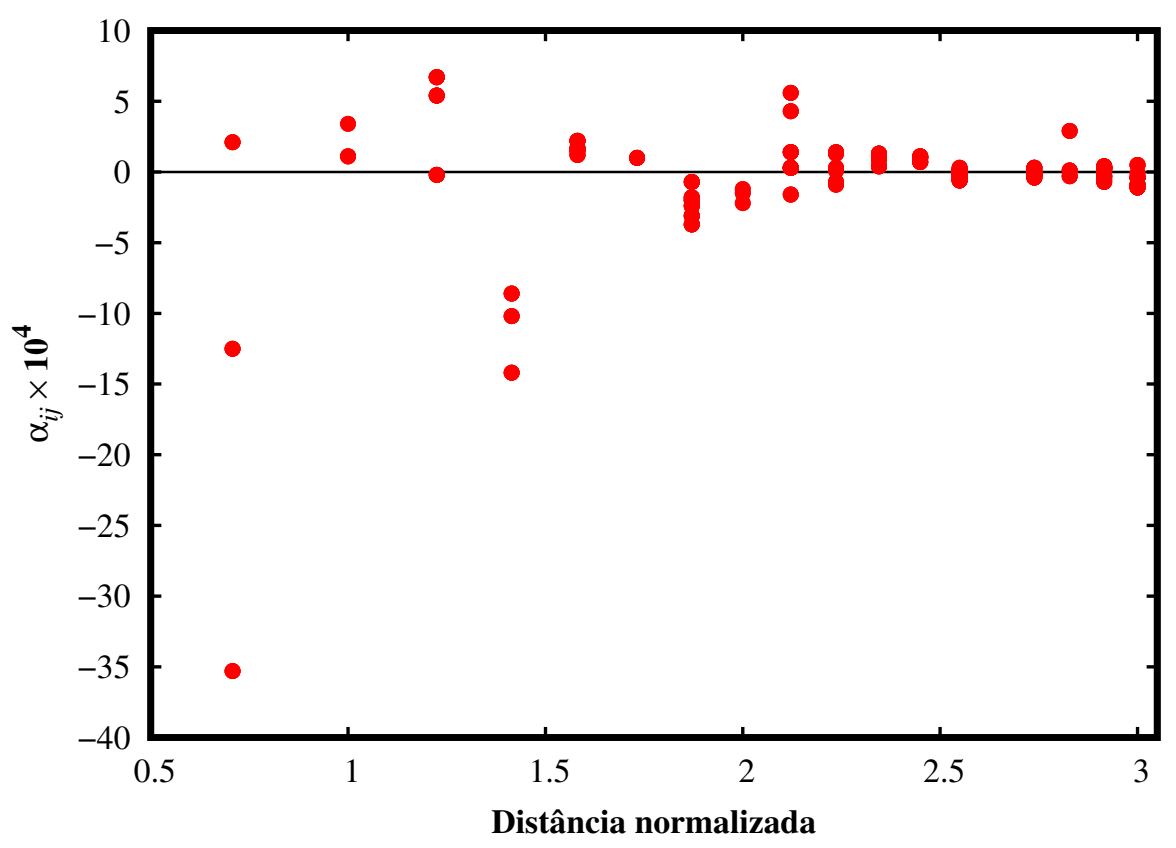

Figura 6.3: Parâmetros de damping de Gilbert não-locais $\left(\alpha_{i j}, i \neq j\right)$ como função da distância normalizada entre os sítios $i$ e $j$, no espaço-real, para o bulk de Ni na estrutura fcc.

Como se pode notar, a contribuição mais significativa ocorre pela influência dos primeiros vizinhos, e, embora existam alguns parâmetros positivos, os valores mais expressivos de $\alpha_{i j}$ tendem a ser negativos, resultando em uma redução global do damping onsite. Ade- 
mais, observa-se, assim como as demais interações magnéticas (tais como o acoplamento de troca $J_{i j}$ e a interação de Dzyaloshinskii-Moriya, $\mathbf{D}_{i j}[48,49]$ ), que as contribuições $\alpha_{i j}$ diminuem com o aumento da distância entre o par $i$ e $j$. A existência de valores negativos para a contribuição de damping não-local foi também observada pelas referências $[225,231]$.

Em divergência ao que foi reportado na referência [225], para o caso da estrutura cúbica de face centrada foram encontrados 3 diferentes valores de damping não-local $\left(\alpha_{\mathrm{nl}}\right)$ entre um sítio típico de Ni e seus 12 primeiros vizinhos; Thonig et al. observaram apenas 2 valores diferentes [225]. A Figura 6.4 mostra o padrão para os valores distintos de $\alpha_{\mathrm{nl}}$, sendo o átomo típico (central) de Ni representado pela cor cinza escuro, e os primeiros vizinhos organizados em diferentes cores para cada resultado de $\alpha_{\mathrm{nl}}$. Assim, observam-se 3 planos com padrões distintos de damping, sejam eles: $x y, x z$, e $y z$. Como estamos considerando uma configuração colinear nesse cálculo, e sendo o momento magnético local do Ni alinhado à direção $z$, a priori deveria existir uma simetria em torno do eixo- $z$ na estrutura bulk $(f c c)$, resultando em dois valores de damping distintos, em razão da presença do acoplamento spin-órbita, e, logo, de um eixo global de quantização de spin: um valor $\boldsymbol{i n}$-plane, no qual os sítios $i$ e $j$ estão localizados no plano $x y$, e um valor $\boldsymbol{o u t}$ of-plane, no qual os sítios estão localizados nos demais planos. Entretanto, podemos identificar que ambos os parâmetros out-of-plane $\alpha_{i j}$ são negativos e apresentam a mesma ordem de magnitude $\left(\alpha_{i j}=-3,525 \times 10^{-3}\right.$ e $\left.\alpha_{i j}=-1,251 \times 10^{-3}\right)$, enquanto o parâmetro in-plane encontrado é o único que apresenta um valor positivo e uma ordem de magnitude menor $\left(\alpha_{i j}=2,053 \times 10^{-4}\right)$. 


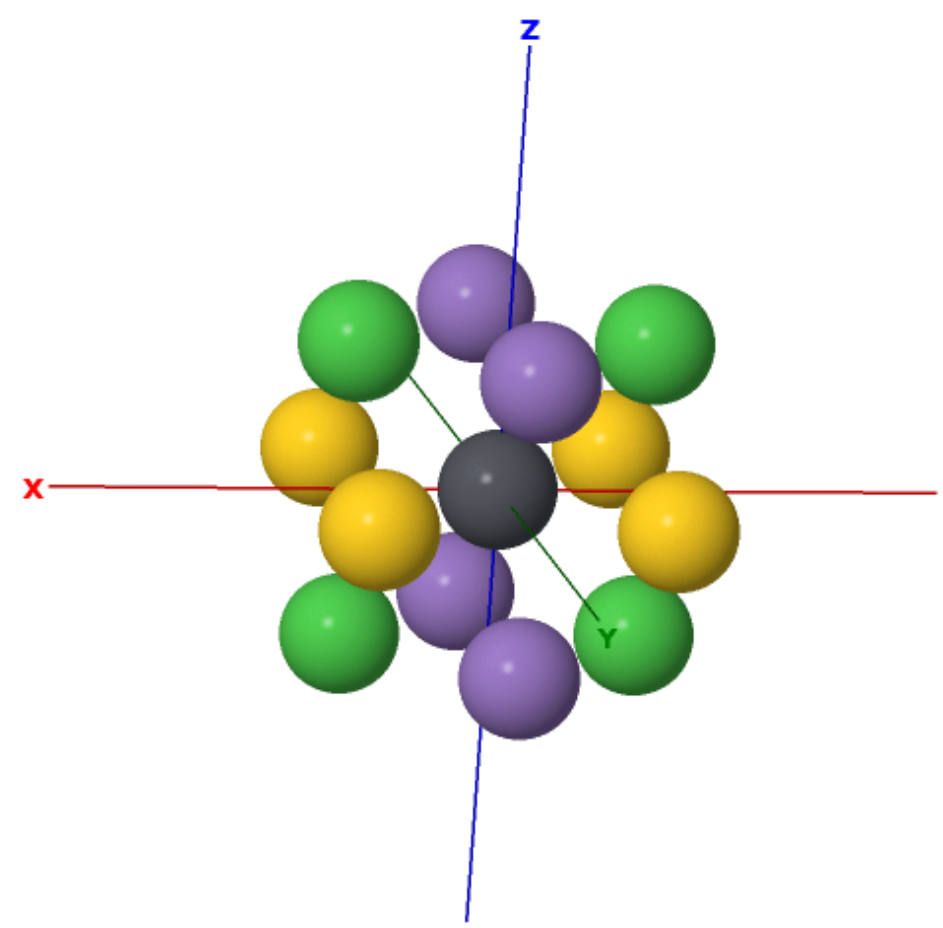

Figura 6.4: Padrão de cores para os diferentes valores de damping não-local $\left(\alpha_{i j}, i \neq j\right)$ calculados via RS-LMTO-ASA entre um átomo de Ni típico e seus primeiros vizinhos da rede $f c c$. Para esse cálculo, utilizou-se $L L=31$ e um cluster no espaço real contendo 66.427 átomos. Cada valor é representado por uma cor, a saber: verde $\left(\alpha_{i j}=-3.525 \times\right.$ $\left.10^{-3}\right)$, roxo $\left(\alpha_{i j}=-1.251 \times 10^{-3}\right)$, e amarelo $\left(\alpha_{i j}=2.053 \times 10^{-4}\right)$.

Esse problema pode ter 3 origens numéricas que serão investigadas ao longo desse capítulo (assumindo-se, inicialmente, não se tratar de um fato físico): ( $i$ ) como cada elemento diagonal função de Green $G_{i j}$ é representado por uma fração continuada [232], truncada pelo terminador $t(\epsilon)$ de Beer-Pettifor [136] (que, por sua vez, está relacionado aos $L L$ níveis de recorrência), e a parte imaginária desses elementos diagonais derivam da parte imaginária de $t(\epsilon)$, então o parâmetro $L L=31$ escolhido ainda não é o ideal para o cálculo do damping não-local entre os sítios $i$ e $j$ - e, nesse caso, uma solução proposta é aumentar o parâmetro $L L$ para aprimorar o valor dos elementos diagonais de $G_{i j}$; $(i i)$ como estamos aproximando o cálculo de um sólido periódico em um cluster (finito) no espaço real, o número de átomos contidos nesse cluster também se relaciona com a estrutura eletrônica final até um certo limite, uma vez que os orbitais obtidos pelo Método de Recorrência de Haydock [135] referem-se aos estados das vizinhanças de um dado sítio - e, logo, melhor serão representados quanto mais longe da borda do cluster estiverem; e (iii) de acordo com o formalismo RS-LMTO-ASA [134], vê-se que cada elemento da matriz de estrutura $\overline{\mathbf{S}}$ depende estritamente dos esféricos harmônicos [138], os quais, por sua vez, são funções do versor $\hat{\mathbf{r}}_{i j}$ entre os sítios $i$ e $j$. Portanto, a matriz de estrutura para cada uma das direções representadas pelas cores roxo (plano $y z$ ), verde (plano $x z$ ) 
e amarelo (plano $x y$ ) na Fig. 6.4 é diferente, pois os esféricos harmônicos diferem entre si $\left(Y_{l}^{m}\left(\hat{\mathbf{r}}_{i j}^{\text {verde }}\right) \neq Y_{l}^{m}\left(\hat{\mathbf{r}}_{i j}^{\text {amarelo }}\right) \neq Y_{l}^{m}\left(\hat{\mathbf{r}}_{i j}^{\text {roxo }}\right)\right)$, resultando em matrizes Hamiltonianas $\mathcal{H}$ distintas e, como consequência, diferentes funções de Green $G_{i j}$. Explicitamente, se tomarmos, por exemplo, o estado $(l, m)=(2,-1)$, teremos que para a estrutura bulk $f c c$ ideal: $\hat{\mathbf{r}}_{i j}^{\text {verde }}=\left\{\left(\frac{1}{\sqrt{2}}, 0, \frac{1}{\sqrt{2}}\right) ;\left(-\frac{1}{\sqrt{2}}, 0, \frac{1}{\sqrt{2}}\right) ;\left(\frac{1}{\sqrt{2}}, 0,-\frac{1}{\sqrt{2}}\right) ;\left(-\frac{1}{\sqrt{2}}, 0,-\frac{1}{\sqrt{2}}\right)\right\}$, que resulta em $Y_{2}^{-1}\left(\hat{\mathbf{r}}_{i j}^{\text {verde }}\right)= \pm \frac{1}{4} \sqrt{\frac{15}{2 \pi}} ; \hat{\mathbf{r}}_{i j}^{\text {roxo }}=\left\{\left(0, \frac{1}{\sqrt{2}}, \frac{1}{\sqrt{2}}\right) ;\left(0,-\frac{1}{\sqrt{2}}, \frac{1}{\sqrt{2}}\right) ;\left(0, \frac{1}{\sqrt{2}},-\frac{1}{\sqrt{2}}\right) ;\left(0,-\frac{1}{\sqrt{2}},-\frac{1}{\sqrt{2}}\right)\right\}$, que resulta em $Y_{2}^{-1}\left(\hat{\mathbf{r}}_{i j}^{\text {roxo }}\right)= \pm \frac{i}{4} \sqrt{\frac{15}{2 \pi}}$, ambos de mesmo módulo (porém valores distintos); e $\hat{\mathbf{r}}_{i j}^{\text {amarelo }}=\left\{\left(\frac{1}{\sqrt{2}}, \frac{1}{\sqrt{2}}, 0\right) ;\left(-\frac{1}{\sqrt{2}}, \frac{1}{\sqrt{2}}, 0\right) ;\left(\frac{1}{\sqrt{2}},-\frac{1}{\sqrt{2}}, 0\right) ;\left(-\frac{1}{\sqrt{2}},-\frac{1}{\sqrt{2}}, 0\right)\right\}$, que resulta em $Y_{2}^{-1}\left(\hat{\mathbf{r}}_{i j}^{\text {amarelo }}\right)=0^{1}$. A origem descrita em $(i)$ será explorada a seguir, para o sistema de Co $f c c$ - e veremos que não explica a diferença observada.

\subsubsection{Bulk de Co fcc}

Analogamente ao realizado para o bulk de Ni fcc anteriormente discutido (incluindose, portanto, o procedimento de calcular o valor total do damping, $\alpha_{\text {tot }}$, por um cluster contendo 459 átomos, como na Fig. 6.2, e utilizando o número de níveis de recorrência $L L=31$ ), porém agora considerando o parâmetro de rede $a=3,61 \AA$ (o mesmo utilizado na referência [225], para fins de comparação, e compatível com o encontrado na referência [233] após otimização estrutural ${ }^{2}$ ), calculou-se os parâmetros de damping para o Co bulk $f c c$ no caso em que os spins estão em uma configuração colinear. Os parâmetros onsite $\left(\alpha_{i i}\right)$ e totais $\left(\alpha_{t o t}\right)$ calculados estão resumidos na Tabela $\mathbf{6 . 2}$.

Tabela 6.2: Contribuição onsite e damping total obtidos para um sítio típico no bulk de Co $f c c$, e comparação com os resultados da literatura.

\begin{tabular}{|l|c|c|}
\hline & Este trabalho & Literatura \\
\hline Onsite $\left(\alpha_{i i}\right)$ & 0,0053 & $0,0036[225]$ \\
\hline Total $\left(\alpha_{\text {tot }}\right)$ & 0,0032 & $0,0057[235] / 0.0045[167]$ \\
\hline
\end{tabular}

Observa-se que os resultados apresentam boa concordância com a literatura, especialmente quando se trata da ordem de magnitude. Como o damping total (Eq. 6.5) é menor que o on-site, pode-se assumir que a contribuição não-local $\left(\alpha_{i j}\right)$ é majoritariamente negativa. Assim, da mesma forma que o desenvolvido na Seção 6.2.1, é interessante analisar como as contribuições não-locais $\left(\alpha_{i j}\right)$ são distribuídas como função da distância

\footnotetext{
${ }^{1}$ É claro que, para existir um valor não-nulo de damping no plano $x y$ (átomos em amarelo na Fig. 6.4), o resultado de alguns esféricos harmônicos é diferente de zero, porém apenas o estado $(l, m)=(2,-1)$ foi utilizado para efeito de comparação.

${ }^{2}$ Valor um pouco maior que o experimental, de $a=3,54 \AA$ [234].
} 
normalizada a partir de um átomo típico de Co na rede $f c c$. Uma comparação entre os resultados obtidos utilizando-se o método RS-LMTO-ASA e o método $\boldsymbol{F}$ ull $\boldsymbol{P}$ otential Linear $\boldsymbol{M}$ uffin-Tin $\boldsymbol{O}$ rbital (FP-LMTO), tal como implementado no pacote RSPt [236], é apresentada na Fig. 6.5. Os resultados via RSPt foram obtidos pelo Dr. Danny Thonig, considerando-se o mesmo sistema, e mesmo parâmetro de rede.

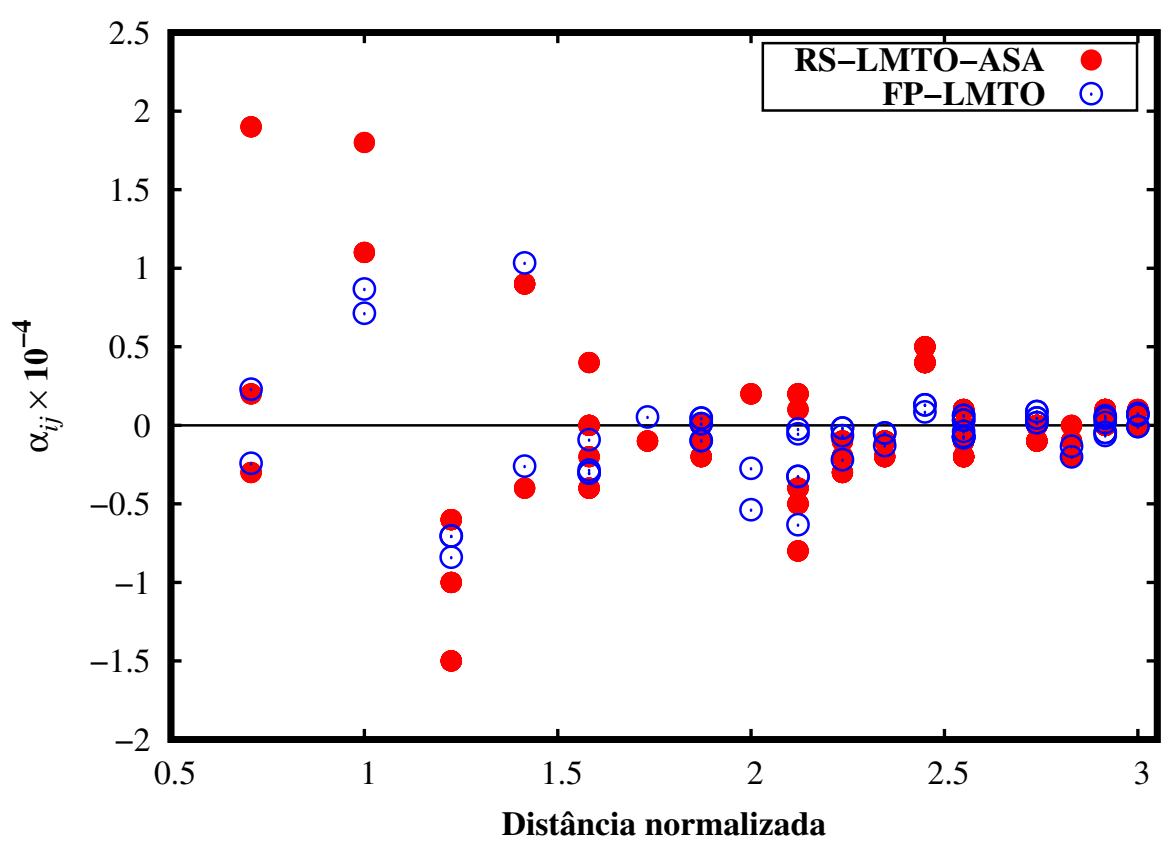

Figura 6.5: Parâmetros de damping de Gilbert não-locais $\left(\alpha_{i j}, i \neq j\right)$ como função da distância normalizada entre os sítios $i$ e $j$ para o bulk de Co na estrutura $f c c$. Em vermelho (círculos preenchidos), os resultados obtidos no espaço-real via RS-LMTO-ASA. Em azul (círculos vazios), os resultados obtidos no espaço-k, via RSPt.

Utilizou-se este sistema para analisar o problema de simetria descrito na Seção 6.2.1, considerando primeiramente o aumento no número de níveis de recorrência $(L L)$. Os valores obtidos para $L L=21,31,41$ das contribuições não-locais $\alpha_{i j}$ entre primeiros vizinhos de um sítio típico de Co no bulk fcc são apresentados na Tabela 6.3. Como se nota, um aumento em $L L$ não provoca uma tendência clara de aproximação entre os termos $\alpha_{i j}$ out-of-plane (para uma referência dos planos, veja a Fig. 6.4). 
Tabela 6.3: Contribuições de damping não-locais $\alpha_{i j}$ entre pares Co-Co primeiros vizinhos $\left(d=\frac{\sqrt{2}}{2} a\right)$ de um sítio típico de Co no bulk fcc como função do número de níveis de recorrência $L L$.

\begin{tabular}{|c|r|r|r|}
\hline $\boldsymbol{\alpha}_{i j}\left(\mathbf{1}^{\text {os }}\right.$ vizinhos $)$ & \multicolumn{1}{c|}{$L L=\mathbf{2 1}$} & \multicolumn{1}{c|}{$L L=\mathbf{3 1}$} & \multicolumn{1}{c|}{$L L=\mathbf{4 1}$} \\
\hline Plano $x z$ (out-of-plane $)$ & $-0,000011$ & 0,000015 & 0,000044 \\
\hline Plano yz (out-of-plane) & $-0,000086$ & $-0,000033$ & $-0,000013$ \\
\hline Plano $x y$ (in-plane) & 0,000142 & 0,000186 & 0,000281 \\
\hline
\end{tabular}

Apesar da quantidade diferente de valores obtidos pelos dois métodos para cada determinada distância (veja a Seção 6.2.1), nota-se que o conjunto de parâmetros obtidos concorda satisfatoriamente bem, reproduzindo com qualidade as tendências observadas para $\alpha_{i j}$ (diminuição com o aumento da distância, existência de só valores positivos ou negativos, ou ambos com um mesmo sinal).

\subsubsection{Bulk de Fe bcc}

O Fe bulk, diferentemente dos dois materiais apresentados anteriormente, cristaliza-se em uma estrutura $b c c$ no estado fundamental (com parâmetro de rede $a=2.87 \AA$ [188]). E é exatamente esta estrutura que dá ao Fe um comportamento ferromagnético espontâneo [194]. Assim, a fim de analisar o comportamento do damping onsite e não-local (Fe-Fe) neste material, considerou-se um cluster no espaço-real com os 339 primeiros vizinhos de um sítio típico de Fe (Fig. 6.6). Assim, o $\alpha_{t o t}$ do Fe de referência é obtido a partir da somatória de todos os dampings (Eq. 6.5) em relação a este sítio. Para isso, considerou-se no processo autoconsistente um total de $L L=31$ níveis recorrência, assim como no caso do Co bulk fcc. Os parâmetros obtidos estão resumidos na Tabela 6.4. 


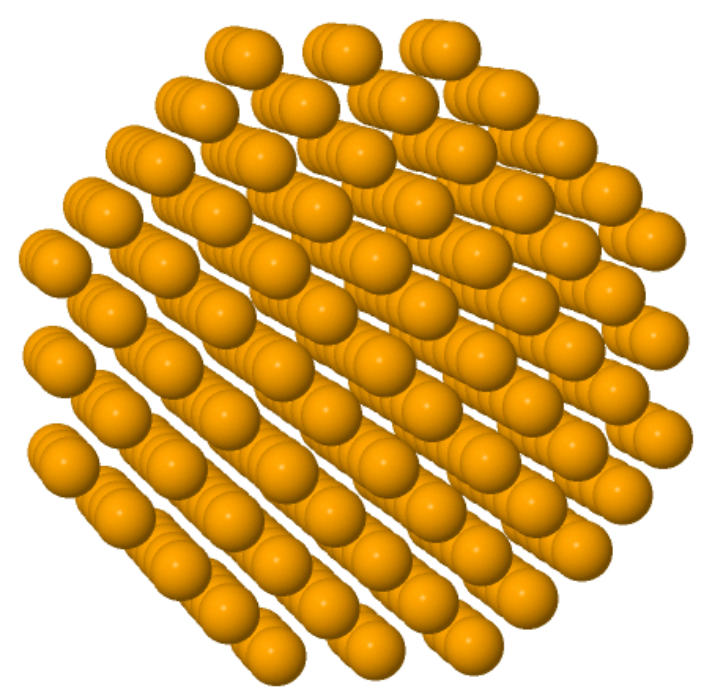

Figura 6.6: Representação esquemática do cluster de Fe no espaço-real, com estrutura bcc e contendo 339 átomos, utilizado no cálculo das contribuições onsite e não-locais do parâmetro de damping de Gilbert.

Tabela 6.4: Contribuição onsite e damping total obtidos para um sítio típico no bulk de Fe $b c c$, e comparação com os resultados da literatura.

\begin{tabular}{|l|c|c|}
\hline & Este trabalho & Literatura \\
\hline Onsite $\left(\alpha_{i i}\right)$ & 0,0016 & $0,0036[225]$ \\
\hline Total $\left(\alpha_{\text {tot }}\right)$ & 0,0042 & $0,0040[225] / 0,0045[237]$ \\
\hline
\end{tabular}

Neste caso, para o $\alpha_{\text {tot }}$ observa-se uma boa concordância com os resultados disponíveis na literatura. A Figura 6.7 mostra uma comparação dos valores de $\alpha_{i j}$ calculados utilizando RS-LMTO-ASA e FP-LMTO, além de apresentar uma comparação com os mesmos resultados obtidos considerando-se o termo de correção de segunda ordem em energia $(\bar{h} \bar{o} \bar{h}$, veja a Eq. B.84 no Apêndice B.1.6). Observa-se que a inclusão do $\bar{h} \bar{o} \bar{h}$ nos cálculos altera pouco o valor dos dampings não-locais Fe-Fe (pontos em verde claro), apresentando valores próximos aos obtidos sem este termo de correção (pontos em vermelho). Da mesma forma que no caso do Co $f c c$, os parâmetros calculados via RSPt (FP-LMTO) são bastante próximos daqueles obtidos via RS-LMTO-ASA. 


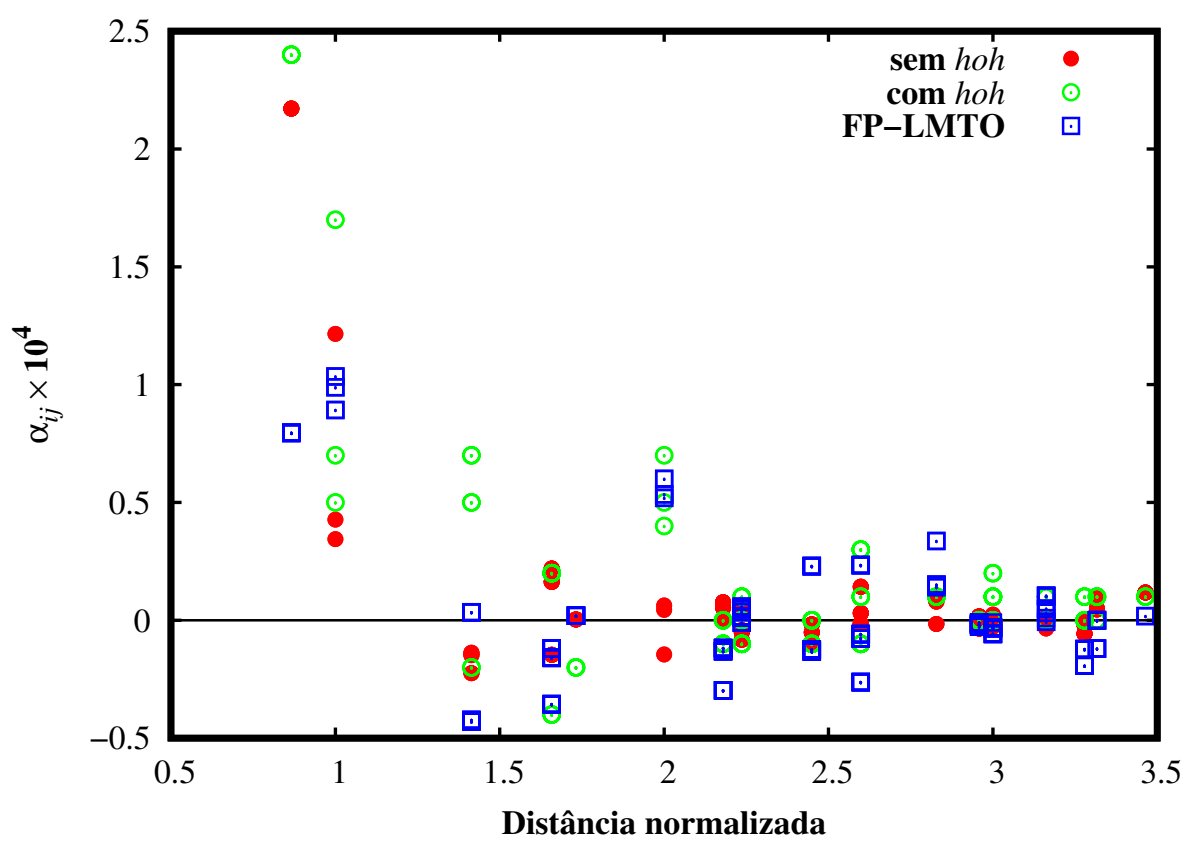

Figura 6.7: Parâmetros de damping de Gilbert não-locais $\left(\alpha_{i j}, i \neq j\right)$ como função da distância normalizada entre os sítios $i$ e $j$ para o bulk de Fe na estrutura bcc. Em vermelho (círculos preenchidos), os resultados obtidos no espaço-real via RS-LMTO-ASA. Em verde claro (círculos vazios), os resultados para o mesmo sistema considerando a correção $\bar{h} \bar{o} \bar{h}$ na Hamiltoniana, utilizando o RS-LMTO-ASA. Em azul (quadrados vazios), os resultados obtidos no espaço-k, via RSPt.

A estrutura $b c c$, mais adiante, fará parte de uma discussão sobre a anisotropia de $\alpha_{\text {tot }}$. Em 2013, Mankovsky et al. [66] reportaram a observação, no bulk de Fe bcc, de uma assimetria entre os parâmetros $\alpha$ quando os cálculos eram realizados em diferentes eixos de magnetização $(\mathbf{M})$ - especialmente para baixas temperaturas. Verificou-se que quando $\mathbf{M} \|$ [110], as componentes $\alpha^{x x}$ e $\alpha^{y y}$ do tensor $\boldsymbol{\alpha}_{i i}$ (Eq. 6.11) diferem por questões de simetria, enquanto para o caso de $\mathbf{M} \|[001]$ as componentes são isotrópicas. Com o aumento da temperatura, essa anisotropia é cada vez mais reduzida. Entretanto, considerando $\alpha$ como uma média entre $\alpha^{x x}$ e $\alpha^{y y}\left(\alpha=\frac{\alpha^{x x}+\alpha^{y y}}{2}\right)$, a anisotropia do damping total para este sistema encontrada na referência [66], assim como discutido mais à frente, é relativamente pequena quando comparada à da superfície de $\mathrm{Fe}_{50} \mathrm{Co}_{50}(100)$, mesmo no limite de baixas temperaturas. 


\subsection{Ligas de $\mathrm{Fe}_{1-x} \mathrm{Co}_{x}$ e $\mathrm{Ni}_{1-x} \mathrm{Fe}_{x}$}

Alguns dos primeiros sistemas investigados na literatura em relação ao comportamento do damping foram as ligas metálicas [65, 68, 167, 235]. Esses estudos foram importantes para delinear a forma com a qual o damping varia em relação à concentração $x$ dos compostos das ligas. Um caso importante foi o da liga de FeCo [167]. Como já é sabido e foi mencionado anteriormente, o damping controla o quão rápido um sistema de spins atinge um eventual equilíbrio termodinâmico, comandando a velocidade e a energia dissipada no processo de relaxação desses spins. Assim, materiais que apresentam baixa constante de damping (i.e., da ordem de $10^{-4}$ ou menos) são interessantes para a aplicações em magnônica e spin-orbitrônica, sendo que uma das aplicações possíveis é o estudo de dinâmica da magnetização ${ }^{1}$ [238]. Nesse contexto, a liga de $\mathrm{Fe}_{1-x} \mathrm{Co}_{x}$ surge como uma proposta de material com damping pequeno. Schoen et al. [167] observaram que, para uma concentração específica de cobalto de $x \sim 25 \%$, o damping da liga atinge um valor mínimo de $(5,0 \pm 1,8) \times 10^{-4}$, coincidindo com a mínima densidade de estados no nível de Fermi $\left(n\left(E_{F}\right)\right)$.

Portanto, o comportamento da liga de FeCo também foi estudado como um benchmark para método de cálculo recém-implementado do damping no espaço-real. Com essa finalidade, e para tentar simular uma estrutura desordenada de $\mathrm{Fe}_{1-x} \mathrm{Co}_{x}$, inicialmente utilizou-se a aproximação Virtual Crystal Approximation (VCA), na qual se considera o cristal com a periodicidade primitiva, porém composto por átomos fictícios (virtuais) que interpolam entre o comportamento dos componentes reais. Na prática, a estrutura bcc do FeCo na aproximação VCA é composta por um único tipo de átomo $3 d$ com $(9-x)$ elétrons de valência ${ }^{2}$. A Figura 6.9 apresenta os valores obtidos, bem como uma comparação com outros resultados publicados na literatura e com um cálculo realizado para a estrutura explícita e ordenada $B 2$ de $\mathrm{Fe}_{50} \mathrm{Co}_{50}$ (Fig. 6.8), cujo damping efetivo foi calculado seguindo a Eq. 6.19.

\footnotetext{
${ }^{1} \mathrm{Um}$ damping baixo representa que o sistema demora mais para atingir o estado final de relaxação, e a dinâmica de magnetização ocorre por um período de tempo maior.

${ }^{2}$ Por exemplo, para uma concentração de $50 \%$ de Fe na liga de FeCo, cada sítio na aproximação VCA é composta por um átomo com $8,5 e^{-}$de valência.
} 


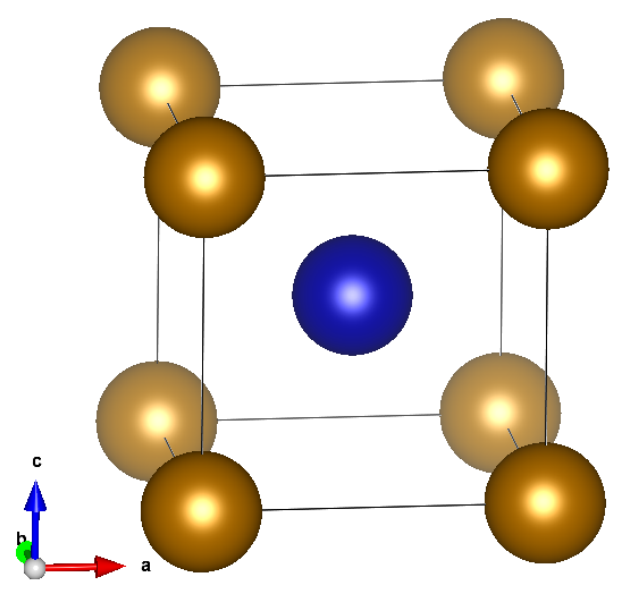

Figura 6.8: Representação esquemática da estrutura ordenada de B2-FeCo $\left(\mathrm{Fe}_{50} \mathrm{Co}_{50}\right)$, com átomos de $\mathrm{Fe}$ (esferas amarelas) nos vértices do cubo e um átomo de Co (esfera azul) no centro, ou vice-versa. Figura produzida utilizando-se o pacote VESTA [239].

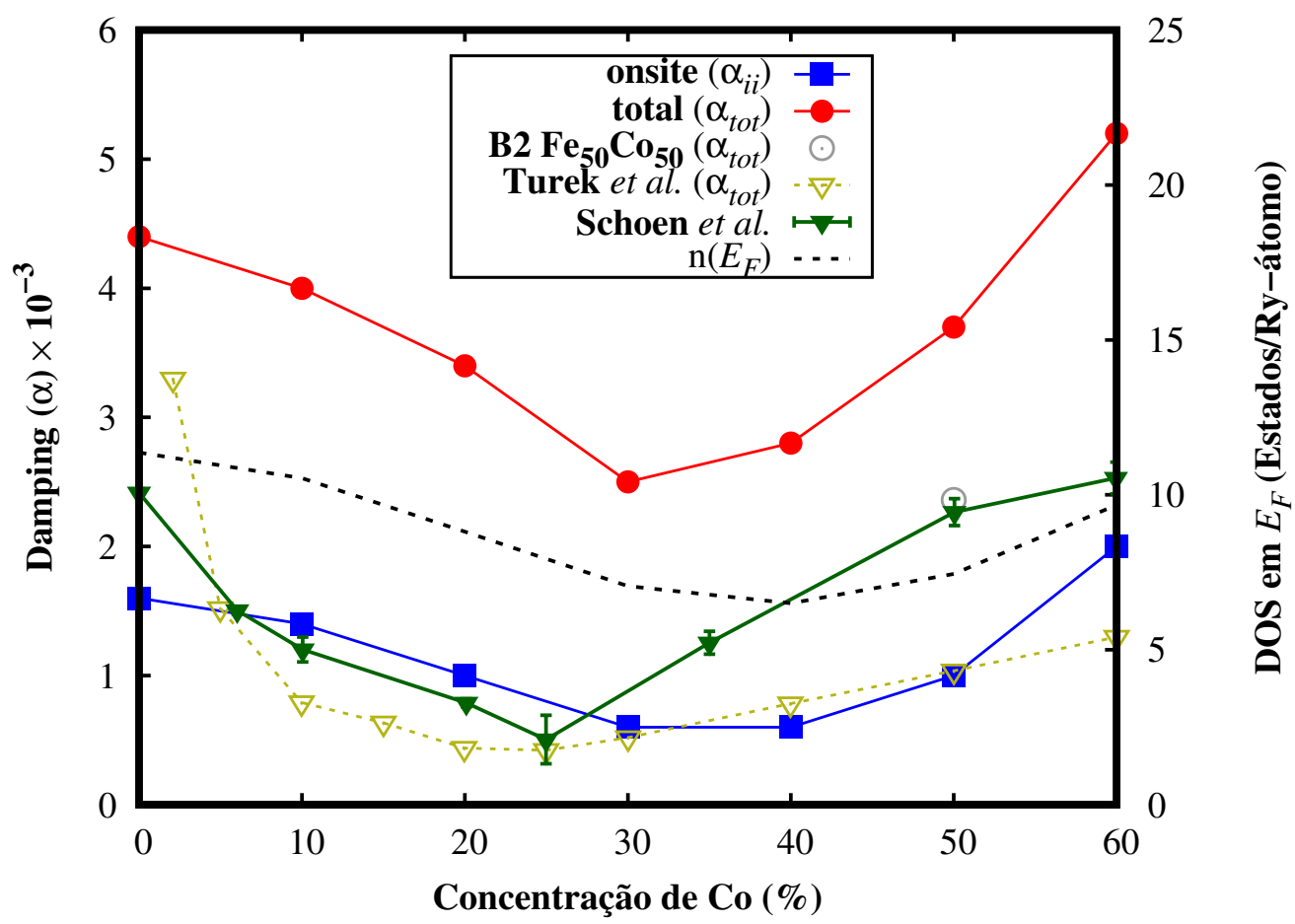

Figura 6.9: Parâmetros de damping de Gilbert onsite $\left(\alpha_{i i}\right)$ e totais $\left(\alpha_{t o t}\right)$ como função da concentração de Co no bulk de $\mathrm{Fe}_{1-x} \mathrm{Co}_{x}$ na estrutura bcc $(a=2,87 \AA)$, na aproximação VCA. Comparação com os resultados experimentais de Schoen et al. [167] e teóricos de Turek et al. [68], bem como com um cálculo realizado para a estrutura explícita e ordenada $B 2$ de $\mathrm{Fe}_{50} \mathrm{Co}_{50}$ (ponto em cinza). A linha preta pontilhada representa a DOS total no nível de Fermi $\left(\left|n^{\uparrow}\left(E_{F}\right)\right|+\left|n^{\downarrow}\left(E_{F}\right)\right|\right)$, obtida com o RS-LMTO-ASA para cada uma das situações. 
É notório que, tanto o comportamento do damping obtido neste trabalho quanto os comportamentos obtidos nas referências $[68,167]$ são compatíveis. Em todos observa-se que há uma concentração de Co (entre $20 \%$ e $30 \%$ ) cujo damping apresenta um mínimo. A partir de $60 \%$ de concentração o sistema deixa de ser bcc. Para o caso do RS-LMTO-ASA, calculou-se um damping mínimo para $x=30 \%\left(\mathrm{Fe}_{70} \mathrm{Co}_{30}\right)$. Como bem é demonstrado na Fig. 6.9, essa mesma tendência obtida para o damping total $\left(\alpha_{t o t}\right)$ é também observada no termo onsite. Além disso, nota-se uma correlação com a DOS total no nível de Fermi, tal qual é conhecida na literatura [240], já que a curva da densidade de estados apresenta a mesma tendência.

Como a aproximação VCA é uma simplificação do sistema, é possível que a consideração de átomos virtuais apresente algumas limitações quando os resultados são confrontados com a experiência. A fim de melhor esclarecer estas eventuais limitações, realizou-se um cálculo do sistema explícito de $\mathrm{Fe}_{50} \mathrm{Co}_{50}$ bcc na estrutura ordenada B2. Nela, os átomos de Fe e Co ocupam, respectivamente, os vértices e o centro da célula cúbica unitária bcc, possuindo 2 átomos na base. Por se tratar de uma estrutura com mais de um tipo atômico, o cálculo do damping total deve seguir a média ponderada da Eq. 6.19, que, neste caso simples, será:

$$
\alpha^{\mathrm{eff}}=\bar{\alpha}_{t o t}=\frac{m_{\mathrm{Fe}} \alpha_{t o t}^{\mathrm{Fe}}+m_{\mathrm{Co}} \alpha_{t o t}^{\mathrm{Co}}}{m_{\mathrm{Fe}}+m_{\mathrm{Co}}} \sim 2,28 \times 10^{-3}
$$

O resultado final para esta concentração foi, então, colocado como um único ponto na Fig. 6.9. Percebe-se que, embora o valor não esteja muito distante daquele obtido com a aproximação VCA, o damping para o FeCo B2 é compatível com o resultado experimental reportado na referência [167]. Pode-se entender, então, que a consideração de átomos virtuais neste sistema aumenta sistematicamente o parâmetro $\alpha_{\text {tot }}$, mas mantém a mesma tendência e resulta em valores não muito distintos dos experimentais.

Outra liga já investigada pela literatura e que foi utilizada como benchmark para validar a implementação do cálculo do damping no espaço-real foi o sistema de $\mathrm{Ni}_{1-x} \mathrm{Fe}_{x}$. De maneira similar ao caso da liga de FeCo, o NiFe foi inicialmente simulado utilizando-se a aproximação VCA. Nesse caso, para uma dada concentração $x$ de Fe na liga, cada átomo apresenta uma valência de $(10-2 x) e^{-}$. O bulk foi simulado por um cluster no espaço-real contendo 16.757 átomos, na estrutura $f c c$. A Figura 6.10, abaixo, apresenta os valores obtidos, bem como uma comparação com os resultados disponíveis na literatura para este sistema. 


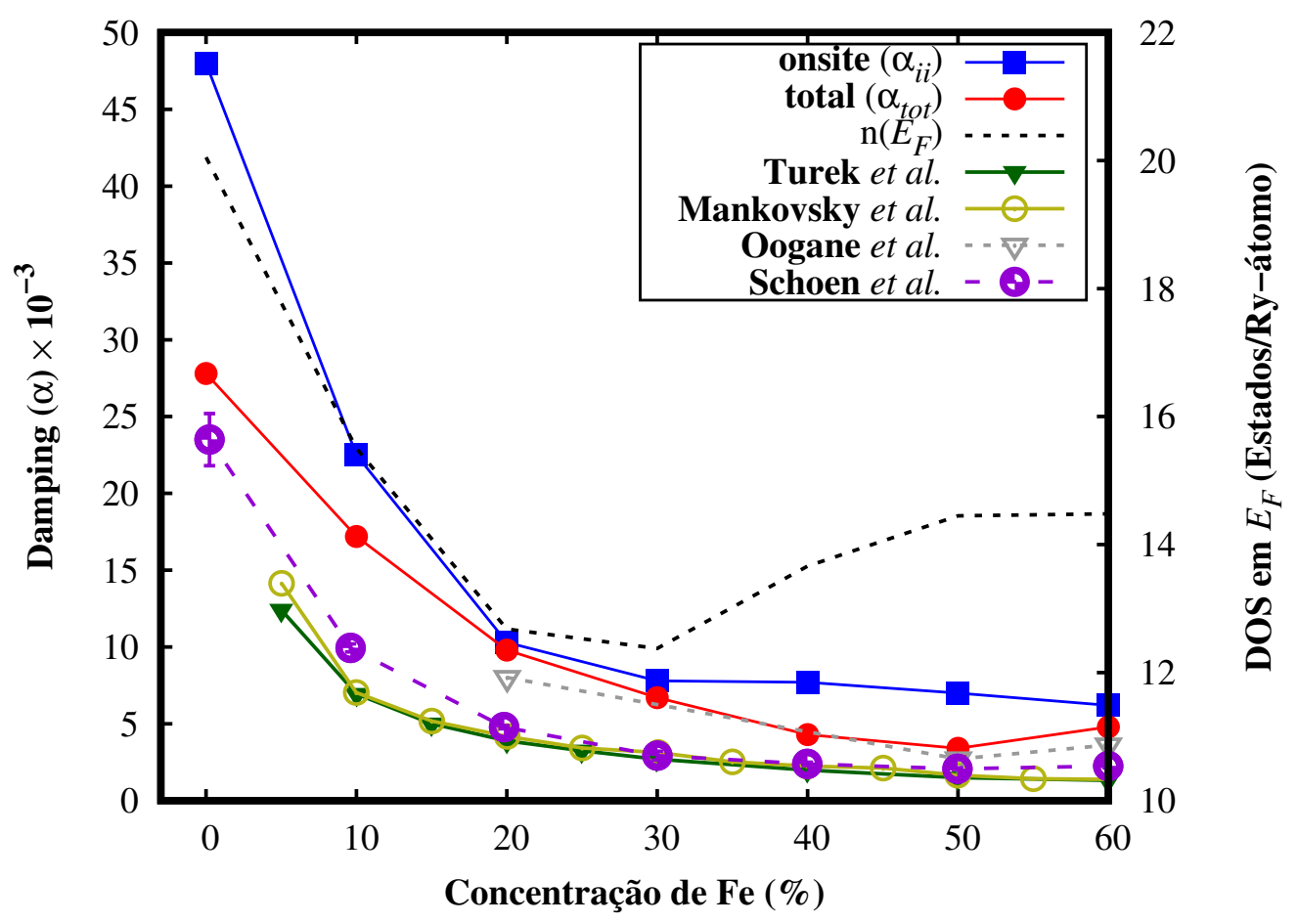

Figura 6.10: Parâmetros de damping de Gilbert onsite $\left(\alpha_{i i}\right)$ e totais $\left(\alpha_{t o t}\right)$ como função da concentração de $\mathrm{Fe}$ no bulk de $\mathrm{Ni}_{1-x} \mathrm{Fe}_{x}$ na estrutura $f c c(a=3,52 \AA)$, na aproximação VCA. Comparação com os resultados teóricos de Turek et al. [68] e Mankovsky et al. [66], e com os resultados experimentais de Oogane et al. [241] e Schoen et al. [228]. A linha preta pontilhada representa a DOS total no nível de Fermi $\left(\left|n^{\uparrow}\left(E_{F}\right)\right|+\left|n^{\downarrow}\left(E_{F}\right)\right|\right)$, obtida com o RS-LMTO-ASA para cada uma das situações.

Neste caso, o damping segue uma tendência monotônica de diminuição, observável em todos os resultados apresentados, teóricos e experimentais. De maneira análoga ao ocorrido com o sistema de $\mathrm{Fe}_{x} \mathrm{Co}_{1-x}$, a liga de $\mathrm{Ni}_{x} \mathrm{Fe}_{1-x}$ tem um damping diretamente proporcional à densidade de estados no nível de Fermi até $~ 30 \%$ de concentração de Fe.

\subsection{Tabela de comparação}

A Tabela 6.5, abaixo, apresenta uma comparação resumida dos resultados obtidos com aqueles disponíveis na literatura.

Como se pode notar, os valores de $\alpha_{\text {tot }}$ e $\alpha_{\text {onsite }}$ obtidos via RS-LMTO-ASA são bastante compatíveis com aqueles apresentados. Isso demonstra a confiabilidade do método 
Tabela 6.5: Damping total (Eq. 6.5) e onsite $\left(\times 10^{-3}\right)$ calculados para alguns sistemas bulk e superfícies, e comparados com os resultados teóricos e experimentais existentes na literatura. A aproximação VCA foi adotada para as ligas, exceto no caso do sistema de $\mathrm{Fe}_{50} \mathrm{Co}_{50}$ bcc na estrutura $B 2$ (veja a Eq. 6.21). As contribuições onsite são indicadas entre parêntesis, enquanto o damping total $\left(\alpha_{t o t}\right)$ é indicado sem qualquer símbolo. Todos os cálculos consideraram o eixo [001] como eixo de magnetização.

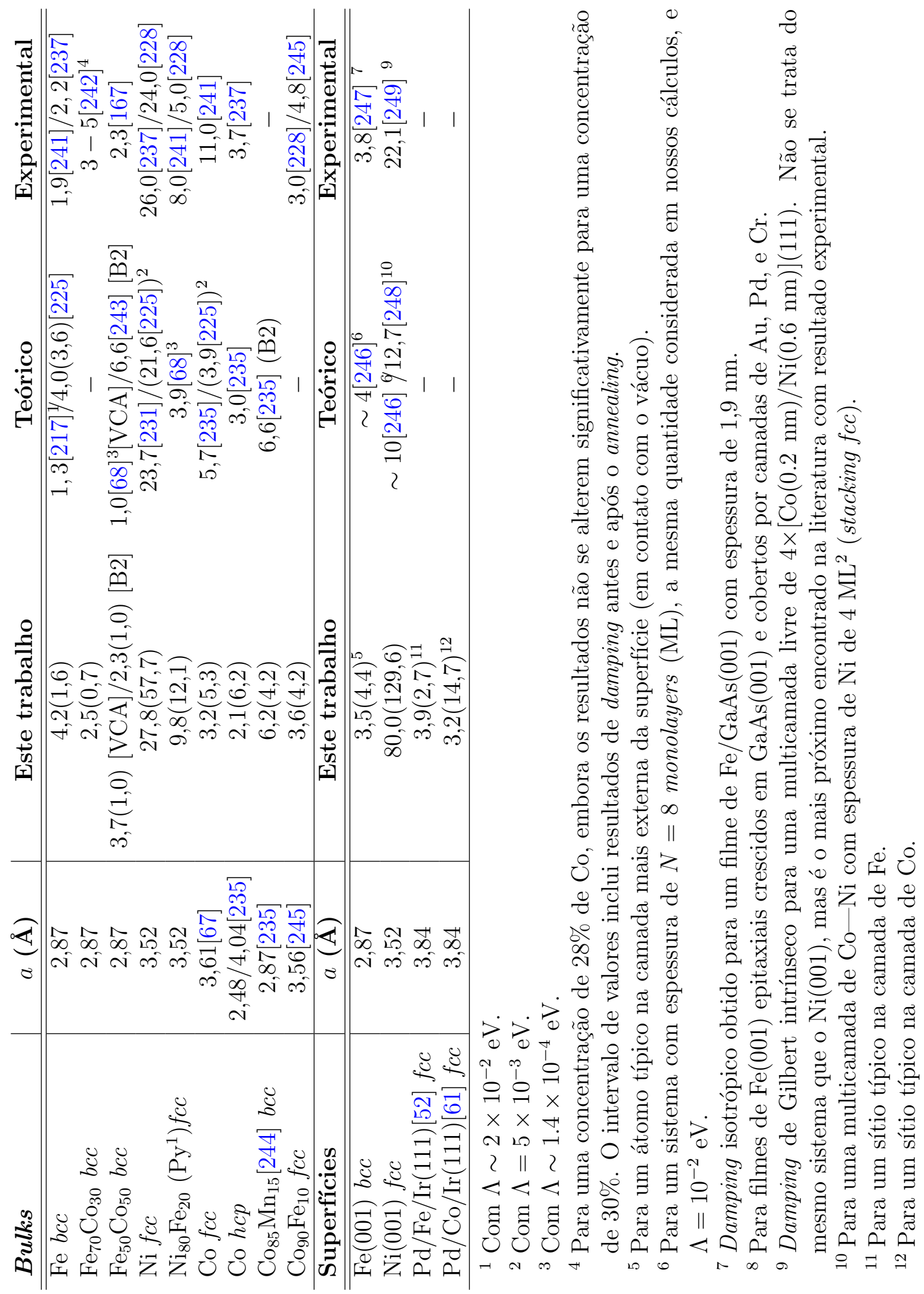


recém-implementado - e, portanto, pronto para a aplicação em sistemas mais complexos no espaço-real, não explorados ainda na literatura.

\subsection{Cálculo do broadening $\left(\Lambda_{R S}\right)$}

Nas seções anteriores, apresentou-se um conjunto de resultados como forma de testar o formalismo para o cálculo do damping recém-implementado no espaço real. Entretanto, como mostram Gilmore et al. [217], Thonig [67], e Guimarães et al. [250], o valor do damping é intimamente dependente do broadening $\Lambda$. Isso porque, como visto na seção 6.1.1, tanto o damping não-local como o damping onsite surgem de transições interbanda e intrabanda, representadas por funções Lorentzianas com largura $\Lambda$, dada em energia. Porém, o broadening "natural" do método RS-LMTO-ASA $\left(\Lambda_{R S}\right)$ boa concordância com a literatura em quase todos os casos analisados (Tabela 6.5), não se mostrando necessário seu ajuste para a realização dos cálculos em diferentes sistemas, distintos daqueles já estudados por outros grupos de pesquisa.

Mesmo assim, é interessante saber qual é o valor de $\Lambda_{R S}$ para poder compará-lo com outros resultados semelhantes presentes na literatura. Sendo assim, o desenvolvimento do método que melhor descreve $\Lambda_{R S}$ também foi um resultado originado desta tese. Muito se discutiu a respeito de como seria possível descobrir o valor do broadening de forma analítica, já que cada elemento da matriz que define a função de Green terá uma parte imaginária distinta. Já o valor de $\Lambda_{R S}$ é definido como aquele único que define a parte imaginária da energia em $\hat{G}(E)=\hat{G}\left(\epsilon+i \Lambda_{R S}\right)$. Logo, uma tentativa possível para se descobrir o valor de $\Lambda_{R S}$ é descrita a seguir.

Sabe-se que a densidade de estados do sistema está relacionada à parte imaginária da função de Green (veja a referência [251], por exemplo):

$$
n(\epsilon)=-\frac{1}{\pi} \mathcal{I} m \operatorname{Tr}\left[\hat{G}\left(\epsilon+i \Lambda_{R S}\right)\right]
$$

sendo a matriz $\hat{G}(E)=(E \mathbb{1}-\mathcal{H})^{-1}$. O valor da Eq. 6.22 é facilmente obtido, já que uma das saídas possíveis do programa é a LDOS $(n(\epsilon))$. Consideremos, a partir de agora, a matriz $\hat{G}\left(\epsilon+i \Lambda_{R S}\right)$. A função de Green apresenta várias propriedades matemáticas convenientes, que permitem sua resolução diretamente sem que seja necessário o cálculo explícito das funções de onda. 
Como a função de Green retardada pode ser escrita, na representação espectral de Lehmann, como (|$\left.\phi_{n}\right\rangle$ são autoestados):

$$
\hat{G}\left(\epsilon+i \Lambda_{R S}\right)=\sum_{n} \frac{\left|\phi_{n}\right\rangle\left\langle\phi_{n}\right|}{\epsilon-\epsilon_{n}+i \Lambda_{R S}}
$$

Então, o imaginário desta matriz é obtido por (basta multiplicar a fração por $\left(\epsilon-\epsilon_{n}-i \Lambda_{R S}\right)$ e obter a parte imaginária):

$$
\mathcal{I} m \hat{G}\left(\epsilon+i \Lambda_{R S}\right)=-\sum_{n} \frac{\Lambda_{R S}}{\left(\epsilon-\epsilon_{n}\right)^{2}+\Lambda_{R S}^{2}}\left|\phi_{n}\right\rangle\left\langle\phi_{n}\right|
$$

Sendo assim, o traço da matriz $\mathcal{I} m \hat{G}$ pode ser obtido por:

$$
\begin{aligned}
\operatorname{Tr} \mathcal{I} m\left[\hat{G}\left(\epsilon+i \Lambda_{R S}\right)\right]= & \mathcal{I} m \operatorname{Tr}\left[\hat{G}\left(\epsilon+i \Lambda_{R S}\right)\right]=-\sum_{i} \sum_{n} \frac{\Lambda_{R S}}{\left(\epsilon-\epsilon_{i n}\right)^{2}+\Lambda_{R S}^{2}}\left\langle\phi_{i} \mid \phi_{n}\right\rangle\left\langle\phi_{n} \mid \phi_{i}\right\rangle \\
& =-\sum_{i} \sum_{n} \frac{\Lambda_{R S}}{\left(\epsilon-\epsilon_{i n}\right)^{2}+\Lambda_{R S}^{2}} \delta_{i n}^{2}=-\sum_{n} \frac{\Lambda_{R S}}{\left(\epsilon-\epsilon_{n}\right)^{2}+\Lambda_{R S}^{2}}
\end{aligned}
$$

Ou seja, a densidade de estados pode ser escrita como um somatório de distribuições de Cauchy:

$$
n(\epsilon)=\sum_{n} \frac{1}{\pi} \frac{\Lambda_{R S}}{\left(\epsilon-\epsilon_{n}\right)^{2}+\Lambda_{R S}^{2}}
$$

onde cada energia $\epsilon_{n}$ pode ser entendida como polos da função de Green quando a parte imaginária da energia é infinitesimalmente pequena $\left(\Lambda_{R S} \rightarrow 0\right)$. Portanto, sendo o valor à direita na Eq. 6.26 obtido pelo cálculo autoconsistente via RS-LMTO-ASA (para uma dada energia $\epsilon$ ), é possível obter $\Lambda_{R S}$ analiticamente.

Outro método possível para a determinação do broadening $\Lambda_{R S}$, e mais simples que o anterior, envolve uma comparação gráfica. Como a implementação do cálculo do damping no método RS-LTMO-ASA permite a escolha livre de valores de $\Lambda$ (veja o Apêndice $\mathbf{D}$ ), uma ideia é obter $\alpha$ para cada diferente valor de $\Lambda$, e comparar com o resultado de $\alpha_{R S}$. Logo, quando a curva $\alpha(\Lambda)=\alpha_{R S}$ teremos encontrado a parte imaginária que melhor representa o valor calculado de damping no espaço-real. 
Realizando este procedimento para o caso do Co fcc (mencionado em detalhes na Seção 6.2.2), e comparando com os resultados disponíveis na literatura, obtém-se o gráfico da Fig. 6.11.

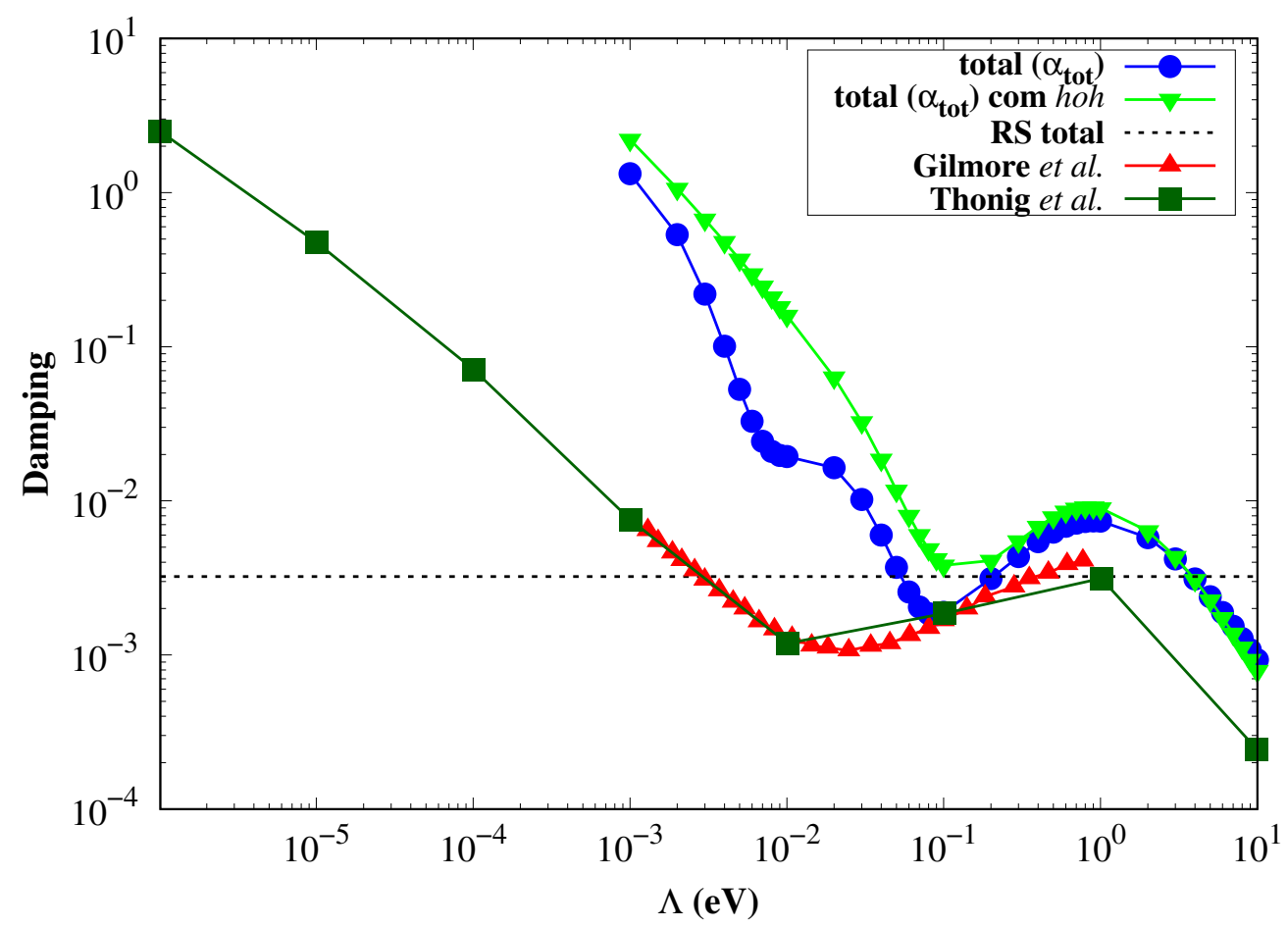

Figura 6.11: Damping calculado para o bulk de Co $f c c(a=3,61 \AA)$ para diferentes valores de broadening $\Lambda$. Resultados obtidos via RS-LMTO-ASA: em azul, os valores de $\alpha_{\text {tot }}$ sem a correção $\bar{h} \bar{o} \bar{h}$, e em verde, os valores de $\alpha_{\text {tot }}$ incluindo a correção $\bar{h} \bar{o} \bar{h}$ na Hamiltoniana. Comparação com os resultados de Thonig et al. [67] e Gilmore et al. [217]. A linha pontilhada representa o valor naturalmente calculado de damping $\left(\Lambda=\Lambda_{R S}\right)$.

Observando a Fig. 6.11, é possível notar claramente que $\Lambda_{R S}$ é pelo menos uma ordem de grandeza maior em energia que os valores $\Lambda$ necessários para atingir o mesmo valor de $\alpha_{t o t}$ com os métodos de Thonig (Tight-Binding) e Gilmore (LAPW [252]) [67, 217], baseados no espaço recíproco. De maneira mais explícita, pelo gráfico obtém-se $\Lambda \sim 3 \times$ $10^{-3} \mathrm{eV}, \Lambda_{R S} \sim 5 \times 10^{-2} \mathrm{eV}$, e $\Lambda_{R S}^{\bar{h} \bar{h} \bar{h}} \sim 10^{-1} \mathrm{eV}$, exigidos para o cálculo de $\alpha_{\text {tot }}=3,2 \times 10^{-3}$. O mesmo padrão se repete para outros sistemas bulk analisados, tais como o Fe bcc. Além disso, o gráfico da Fig. 6.11 deixa claro que há três valores que produzem o mesmo $\alpha_{\text {tot }}$, já que a curva $\alpha(\Lambda)$ coincide duas vezes com a linha horizontal pontilhada. Esses valores podem ser calculados pela Eq. 6.26. Entretanto, seguindo o adotado por Thonig e Gilmore, escolhe-se o menor deles para representar $\Lambda_{R S}$. 


\subsection{Damping nas superfícies de $\mathrm{Pd} / \mathrm{Fe}$ e $\mathrm{Pd} / \mathrm{Co}$ em $\operatorname{Ir}(111)$}

Realizados alguns testes e conhecidas as principais características do damping obtido no espaço-real, iniciaremos a partir desta seção os cálculos em sistemas nunca antes explorados na literatura para esta propriedade. É sabido [221], entretanto, que o método utilizado aqui (adiabático, baseado na fórmula de Kamberský) tem limitações devido à presença de um elevado acoplamento spin-órbita, originado do Pd e do Ir nos sistemas ${ }^{1}$.

Considerando os tópicos anteriormente apresentados, dois sistemas para os quais a aplicação do método recém-implementado é natural são as superfícies de $\mathrm{Pd} / \mathrm{Fe} / \mathrm{Ir}(111)$ e $\mathrm{Pd} / \mathrm{Co} / \mathrm{Ir}(111)$. Os resultados obtidos por primeiros princípios demonstraram algumas causas da não-colinearidade espontânea da superfície com Fe (e o consequente surgimento de skyrmions neste sistema, quando aplicado um campo magnético externo), cuja manifestação física é distinta daquela apresentada pela superfície com Co (configuração colinear). Sendo o damping uma característica fundamental para a dinâmica de spins, como este se manifesta em cada uma dessas superfícies?

Para isso, utilizou-se o mesmo cluster representado na Fig. 5.2(b), em ambos os casos. Assim, para que se obtivesse um valor de damping total para cada átomo típico de Fe ou Co, calculou-se o somatório definido pela Eq. 6.5, em que os valores onsite e não-locais em relação ao sítio de referência foram considerados por todo o cluster. Por sua vez, este sítio de referência foi considerado como aquele que está exatamente no centro da camada de Fe ou Co, a fim de se manter a simetria no somatório para $\alpha_{\text {tot }}$. A Tabela 6.6, a seguir, apresenta os valores de $\alpha_{\text {tot }}$ e $\alpha_{\text {onsite }}$ para cada átomo típico de Fe e Co nas multicamadas de $\mathrm{Pd} / \mathrm{Fe} / \mathrm{Ir}(111)$ e $\mathrm{Pd} / \mathrm{Co} / \operatorname{Ir}(111)$, respectivamente.

Tabela 6.6: Valores de damping $\left(\times 10^{-3}\right)$ calculados para cada átomo típico de Fe ou Co nas superfícies de $\mathrm{Pd} / \mathrm{Fe} / \mathrm{Ir}(111)$ e $\mathrm{Pd} / \mathrm{Co} / \mathrm{Ir}(111)$, nesta ordem.

\begin{tabular}{|c|c|c|}
\cline { 2 - 3 } \multicolumn{1}{l|}{} & $\boldsymbol{\alpha}_{\text {onsite }}$ & $\boldsymbol{\alpha}_{\text {tot }}$ \\
\hline Fe & 2,7 & 3,9 \\
\hline Co & 14,7 & 3,2 \\
\hline
\end{tabular}

Como mostra a tabela, a superfície de Co apresenta um damping onsite maior que a su-

\footnotetext{
${ }^{1}$ Uma melhoria destes resultados, utilizando o formalismo reportado na referência [221] é uma das perspectivas futuras deste trabalho.
} 
perfície de Fe, como esperado ${ }^{1}$. Entretanto, este damping é diminuído significativamente pela influência não-local. Em uma primeira análise, isso significa, em termos de dinâmica de spins, que estruturas magnéticas em ambas as multicamadas de $\mathrm{Pd} / \mathrm{Fe} / \mathrm{Ir}(111)$ e $\mathrm{Pd} / \mathrm{Co} / \operatorname{Ir}(111)$ tendem a demorar o mesmo tempo para atingirem o equilíbrio (ou sua configuração final).

Aproveitando as facilidades trazidas pelo método RS-LMTO-ASA para o tratamento de sistemas sem simetria de inversão, e tendo em vista que, em uma situação experimental, é possível que algumas impurezas de Ir ou de Pd localizem-se na superfície de Fe ou de Co, também analisou-se a influência local destas impurezas no damping. Neste caso, há uma localização do ambiente com elevado acoplamento spin-órbita. Os resultados obtidos serão discutidos logo a seguir.

\subsubsection{Influência de impurezas de Ir $(5 d)$ e Pd (4d) no damping local}

Do ponto de vista experimental, como já comentado, a existência de impurezas na amostra pode se tornar inevitável. Isso tem estimulado uma série de estudos a respeito da interação de estruturas magnéticas complexas, tais como skyrmions, [198, 200, 253, 254] com essas impurezas (ou defeitos). Dessas interações, sabe-se que os skyrmions podem ficar definitivamente presos a esses defeitos (pinning), sofrer atração ou repulsão aos centros de pinning (dependendo de qual é a impureza) [199], ou mover-se prendendo-se e desprendendo-se dos centros de pinning por ação de flutuações térmicas e/ou quânticas (creep-like motion [199]). Inclusive a literatura tem proposto ideias inovadoras como a utilização dessas interações com impurezas, tais como repulsão e pinning de skyrmions, para a criação de caminhos e portas lógicas [253] (veja a Figura 6.12).

\footnotetext{
${ }^{1}$ É conhecido que o Co apresenta um momento orbital maior que o Fe, e, como o damping é fundamentalmente relacionado à interação spin-órbita, é de se esperar que, em uma mesma estrutura e simetria, o Co apresente também um maior damping.
} 


\section{(a) Fios repulsivos}

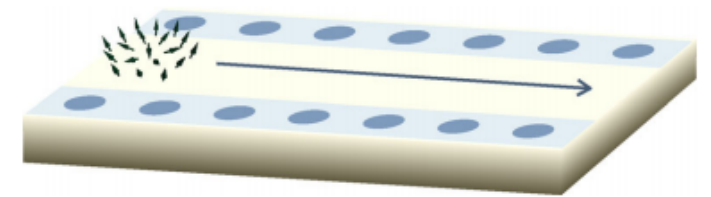

(b) Fio de pinning

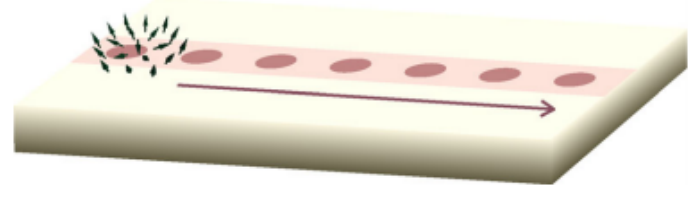

Figura 6.12: Impurezas como guias do movimento de skyrmions: (a) como centros repulsivos, confinando o skyrmion em uma região desejada; (b) como centros de pinning, responsáveis por desacelerar o skyrmion (diminuindo, assim, sua mobilidade). Adaptado da referência [253].

Dada a importância de se conhecer as propriedades magnéticas locais em regiões próximas às impurezas, uma proposta interessante de utilização do método de cálculo de damping recém-implementado é a de se verificar os efeitos de átomos de Ir ou Pd nas camadas magnéticas. Para isso, escolheu-se a superfície de $\mathrm{Pd} / \mathrm{Fe} / \mathrm{Ir}(111)$, por se tratar de um sistema com especial relevância nesta área, e para o qual já foram apresentados resultados de dinâmica de skyrmions próximos a defeitos via RS-LMTO-ASA (Seção 5.4). Nela, as impurezas de Ir e Pd foram colocadas na camada de Fe, considerando-se, para o cálculo no espaço-real, clusters como os apresentados na Figura 6.13. Os dampings calculados referem-se a um átomo de Fe primeiro vizinho da impureza. Para se chegar ao valor de $\alpha_{\text {tot }}$ (Eq. 6.5), considerou-se a soma de $\alpha_{\text {onsite }}$ com todos os termos não-locais $\left(\alpha_{i j}\right)$ em relação aos sítios ao redor, até uma distância de $3 a$. 


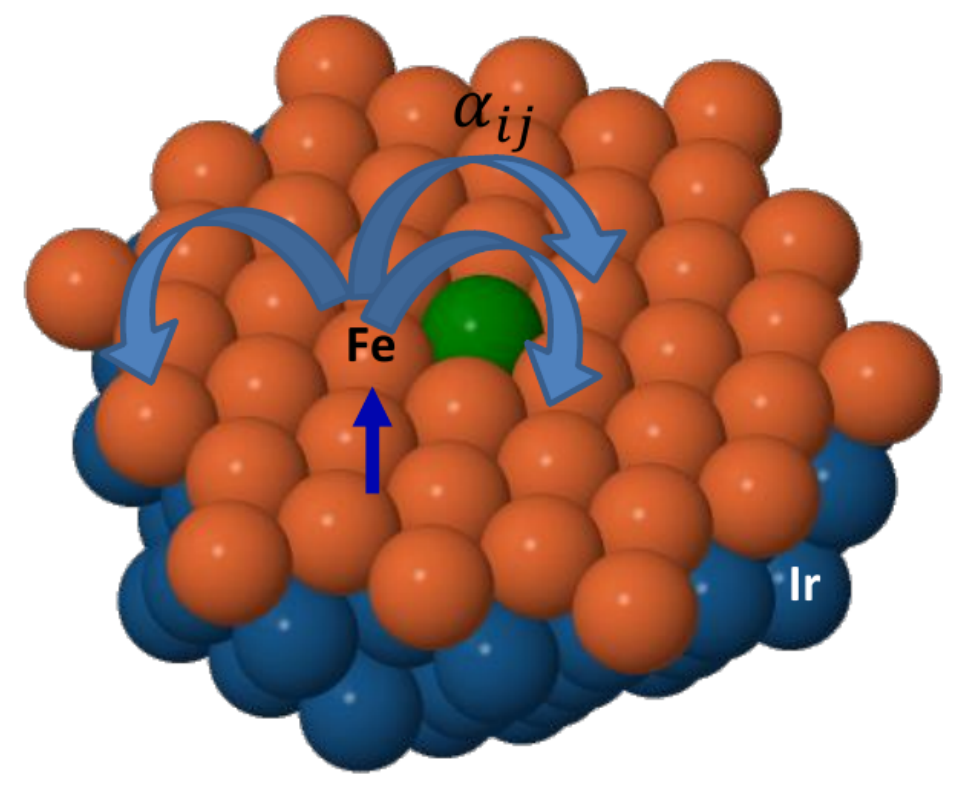

Figura 6.13: Representação esquemática do cluster da superfície de $\mathrm{Pd} / \mathrm{Fe} / \operatorname{Ir}(111)$, no espaço-real, utilizado para a investigação da influência de impurezas no damping do Fe. O cluster foi construído considerando-se uma distância de $3 a$ a partir de um átomo típico (de referência) na camada de Fe. Embora estejam representadas apenas as camadas de Fe e Ir, há uma camada de $\mathrm{Pd}$ mais externa (empilhamento $f c c$ ), que foi ocultada para facilitar a visualização. O átomo de referência (em verde) foi substituído por uma impureza de Pd ou Ir. A seta indica o sítio de Fe (primeiro vizinho) escolhido para o cálculo de $\alpha_{\text {onsite }}$ e $\alpha_{t o t}$.

Tabela 6.7: Valores de damping $\left(\times 10^{-3}\right)$ calculados para um sítio de Fe primeiro vizinho da impureza (Fig. 6.13) na superfície de $\mathrm{Pd} / \mathrm{Fe} / \mathrm{Ir}(111)$. Os tipos atômicos apresentados referem-se aos defeitos de: Fe (superfície sem impurezas), Pd, ou Ir.

\begin{tabular}{|c|c|c|}
\cline { 2 - 3 } \multicolumn{1}{c|}{} & $\boldsymbol{\alpha}_{\text {onsite }}$ & $\boldsymbol{\alpha}_{\text {tot }}$ \\
\hline $\mathbf{F e}$ & 2,7 & 3,9 \\
\hline $\mathbf{P d}$ & 3,0 & 4,2 \\
\hline $\mathbf{I r}$ & 3,7 & 5,1 \\
\hline
\end{tabular}

A inclusão dessas impurezas modificou o damping local de átomos de Fe próximos. Os resultados estão sistematizados na Tabela 6.7. Nota-se que, provavelmente devido ao elevado spin-órbita dos átomos de $\mathrm{Pd}(4 d)$ e, sobretudo, de $\operatorname{Ir}(5 d)$, o valor de $\alpha_{\text {tot }}$ para um átomo de Fe próximo ao defeito aumentou consideravelmente. A comparação entre os valores de $\alpha_{\text {onsite }}$ e $\alpha_{\text {tot }}$ em todos os casos demonstra que o aumento significativo de $\alpha_{\text {tot }}$ nas regiões próximas aos defeitos é influenciada sobretudo por mudanças no damping onsite. Entretanto, apesar de não alterar de maneira significativa a contribuição final advinda do damping não-local, ainda há alterações em $\alpha_{i j}$, devidas à presença do defeito, 
que valem a pena ser destacadas. Essas mudanças são exibidas na Fig. 6.14, na qual se apresenta o somatório das contribuições não-locais de damping entre pares Fe-Fe na multicamada $\mathrm{Pd} / \mathrm{Fe} / \mathrm{Ir}(111)$ para uma mesma distância do sítio de referência.

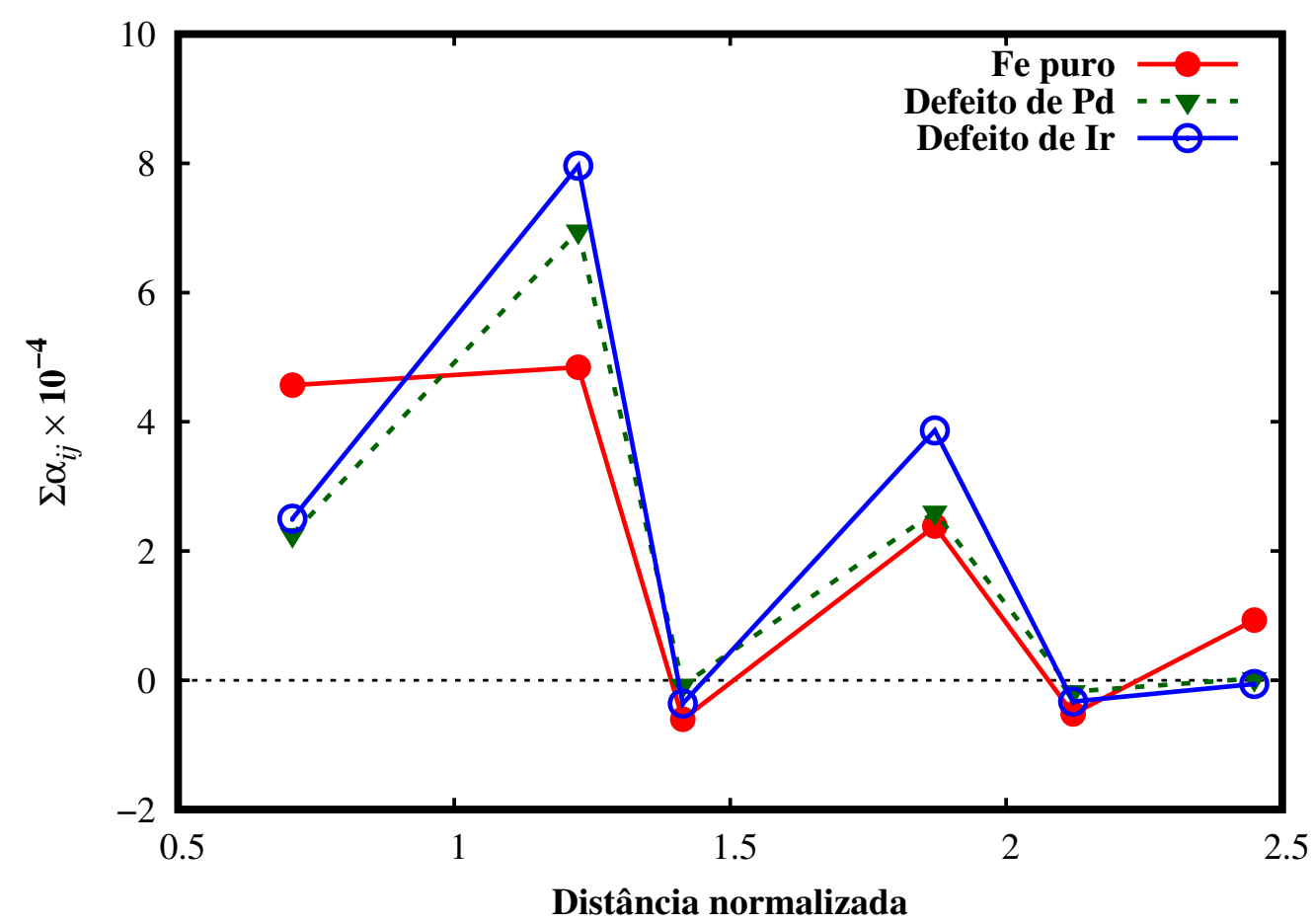

Figura 6.14: Somatório das contribuições não-locais Fe-Fe para o damping $\left(\alpha_{i j}\right)$ para cada distância normalizada na multicamada $\mathrm{Pd} / \mathrm{Fe} / \mathrm{Ir}(111)$, nos casos: da camada de $\mathrm{Fe}$ pura (círculos vermelhos); da camada de Fe com um defeito de Pd (triângulos verdes); e da camada de Fe com um defeito de Ir (círculos azuis vazios). O sítio de Fe considerado é o mesmo em todos os casos, sendo este vizinho do Pd ou do Ir nos sistemas que apresentam defeitos. As linhas são guias para os olhos.

Isso significa que uma estrutura magnética que esteja se movendo pela camada de Fe, ao se encontrar com um defeito de Ir ou Pd, passará por uma região de maior dissipação de energia (ou amortecimento do movimento dos spins), acarretando em uma menor velocidade de deriva para uma dada densidade de corrente, $\mathbf{j}$, aplicada. Assim, de certa forma, o aumento do damping contribuirá para que esses defeitos atuem como centros de pinning de skyrmions ou de paredes de domínio, tal como foi obtido para um defeito de Ir na camada de Fe da multicamada Pd/Fe/Ir(111) (Seção 5.4). Entretanto, no caso do defeito de $\mathrm{Pd}$ o aumento de $\alpha_{\text {tot }}$ não é tão substancial, e este resultado corrobora com o reportado por Fernandes et al. [253], que verificou um caráter quase inerte do Pd como centro de pinning. Portanto, nossos resultados sugerem que a natureza da interação 
de impurezas com estruturas magnéticas como skyrmions também está relacionada à mudança local do damping - e, logo, simulações mais completas e realistas deste tipo de interação devem considerar $\alpha$ como um parâmetro de caráter atomístico, mais do que uma quantidade média (global) do sistema.

\subsection{Anisotropia de damping: o caso da superfície de $\mathrm{Fe}_{50} \mathrm{Co}_{50}(100)$}

Recentemente, Y. Li et al. [255] reportaram na literatura a observação experimental de uma anisotropia de damping gigante (de $\sim 400 \%$ do valor mais baixo encontrado) em filmes finos de $\mathrm{Fe}_{50} \mathrm{Co}_{50}$ depositados em $\mathrm{MgO}(100)$. De acordo com os autores, essa anisotropia foi observada ao se aplicar um campo magnético de bias em diferentes direções. Como é explicado no trabalho original, o crescimento de filmes de $\mathrm{Fe}_{50} \mathrm{Co}_{50}$ (bcc) em substratos de $\mathrm{MgO}(100)$ ocorre de tal forma que essas estruturas cristalinas crescem com uma angulação de $45^{\circ}$ e incompatibilidade (mismatch) de parâmetros de rede de $-4,3 \%$ [256]. Esse tipo de observação é relevante para a literatura, pois sugere um controle direto sobre essa propriedade magnética, com possíveis aplicações futuras em dispositivos, tal como ocorreu com o efeito de magnetoresistência gigante [257], observado em 1988. Os resultados reportados a seguir estão disponíveis na referência [220], e foram submetidos para a publicação. Neste caso, por não tratar de materiais com elevado acoplamento spinórbita (nominalmente Fe e Co), não estão sujeitas às limitações ao método, apresentadas na referência [221].

Na referência [255], os autores observaram diversas anisotropias diferentes entre as direções [010] e [110], dependendo da espessura e da configuração dos sistemas analisados. Por exemplo, para o caso de filmes de $\mathrm{Fe}_{50} \mathrm{Co}_{50} / \mathrm{MgO}(100)$ com $20 \mathrm{~nm}$ de espessura, observou-se um aumento de $\alpha=5,4 \times 10^{-3}$ para $\alpha=24,0 \times 10^{-3}$ com a variação em $45^{\circ}$ do campo magnético externo aplicado - um crescimento substancial de $340 \%$. Por outro lado, para o caso de filmes de $\mathrm{Fe}_{50} \mathrm{Co}_{50}(10 \mathrm{~nm}) / \mathrm{Pt}(6 \mathrm{~nm}) / \mathrm{MgO}(100)^{2}$, observou-se um aumento do damping de $\alpha=3,2 \times 10^{-3}$ para $\alpha=12,2 \times 10^{-3}$ (já desconsiderando-se a contribuição da Pt), que representa um crescimento de $280 \%$. Essa anisotropia de $\alpha$ sempre acompanha a anisotropia cúbica cristalina do bulk, e apresenta um comportamento distinto daquele observado em superfícies puras, como a de $\mathrm{Fe}(001)$ bcc [247, 255]. É

\footnotetext{
${ }^{1}$ Isso faz com que as direções de magnetização na referência [255] estejam $45^{\circ}$ rotacionadas em relação às direções nominais (veja também a referência [256]).

${ }^{2} \mathrm{~A} \mathrm{Pt}$, neste caso, foi utilizada para se aumentar o efeito de spin-transfer-torque (STT), por causa do seu elevado spin-órbita.
} 
interessante notar que não se trata de uma concentração especial de FeCo em relação ao damping, já que o mínimo de $\alpha$ é atingido para uma concentração de $\sim 30 \%$ de Co (veja a Fig. 6.9).

Os autores explicam este efeito com base, principalmente, na variação local de spinórbita em diferentes direções cristalinas (podendo inclusive estar relacionadas à distorções locais do tipo tetragonal na liga de FeCo [258]). O acoplamento spin-órbita (ASO) está diretamente relacionado ao damping em metais de transição pelo modelo BFS. Entretanto, os cálculos ab-initio realizados pelo grupo de pesquisa na referência [255] foram apenas relacionados à estrutura eletrônica da superfície de $\mathrm{Fe}_{50} \mathrm{Co}_{50}(100)$, não investigando mais a fundo a origem da anisotropia de $\alpha$ observada.

Assim, levando-se em conta a recente implementação do cálculo de damping no espaçoreal, foi proposto analisar as origens da elevada anisotropia de $\alpha$ observada experimentalmente para esse sistema. Considerando os resultados apresentados na Fig. 6.9 e a neutralidade da superfície de $\mathrm{MgO}$ nas propriedades magnéticas do filme de $\mathrm{Fe}_{50} \mathrm{Co}_{50}$, o processo foi iniciado pela construção de uma superfície de $\mathrm{Fe}_{50} \mathrm{Co}_{50}(100)$ no modelo VCA. O sistema no espaço-real contou com 4488 átomos, e 7 camadas não-equivalentes de uma liga de $\mathrm{FeCo}\left(8,5 e^{-}\right.$de valência), além de 1 camada de esferas vazias (a fim de simular o vácuo), necessárias para uma correta convergência da estrutura eletrônica. Esse processo se deu nos mesmos moldes do realizado anteriormente com as superfícies de $\mathrm{Pd} / \mathrm{Fe} / \mathrm{Ir}(111)$ e $\mathrm{Pd} / \mathrm{Co} / \operatorname{Ir}(111)$ (veja a Seção 5.1 e a Fig. 3.4). Nos filmes de $\mathrm{Fe}_{50} \mathrm{Co}_{50}$ orientados na direção (100) (out-of-plane) espera-se uma relaxação estrutural bastante pequena $(\sim 0,1 \%[256])$, e, portanto, seus efeitos nas análises de damping realizadas nesta Seção são desprezíveis.

Em suma, foi necessária a construção dos seguintes modelos:

- Estruturas ordenadas de $\mathrm{Fe}_{50} \mathrm{Co}_{50}$ na simetria cúbica B2 (Fig. 6.8), posteriormente referido como B2-FeCo, com parâmetro de rede $a=2,84 \AA$ (otimizado via Quantum ESPRESSO [180, 181]);

- Ligas aleatórias (aproximação VCA) de $\mathrm{Fe}_{50} \mathrm{Co}_{50}$ na estrutura bcc ou bct, com $\frac{c}{a}=$ 1,15 ou $\frac{c}{a}=1,09$, dependendo do caso, e parâmetro de rede igual ao do Fe bulk (bcc), de $a=2,87 \AA^{1}$;

\footnotetext{
${ }^{1}$ Essa escolha foi baseada em dois fatores: $(i)$ uma maior facilidade de comparação dos resultados de damping entre o $\mathrm{VCA} \mathrm{Fe}_{50} \mathrm{Co}_{50} b c c$ e o $\mathrm{Fe} b c c$; e (ii) o fato de os resultados de damping para a liga de FeCo, considerando $a=2,87 \AA$, se mostrarem confiáveis em relação aos dados experimentais disponíveis (veja a Fig. 6.9).
} 
- Ligas de $\mathrm{Fe}_{50} \mathrm{Co}_{50}$ que foram aproximadas como clusters explícitos no espaço-real embebidos em um substrato VCA (com valência de 8,5 elétrons), na estrutura $b c c$ ou bct (com $\frac{c}{a}=1,15$ ou $\frac{c}{a}=1,09$, dependendo do caso). Uma representação esquemática deste modelo é apresentada na Fig. 6.15.
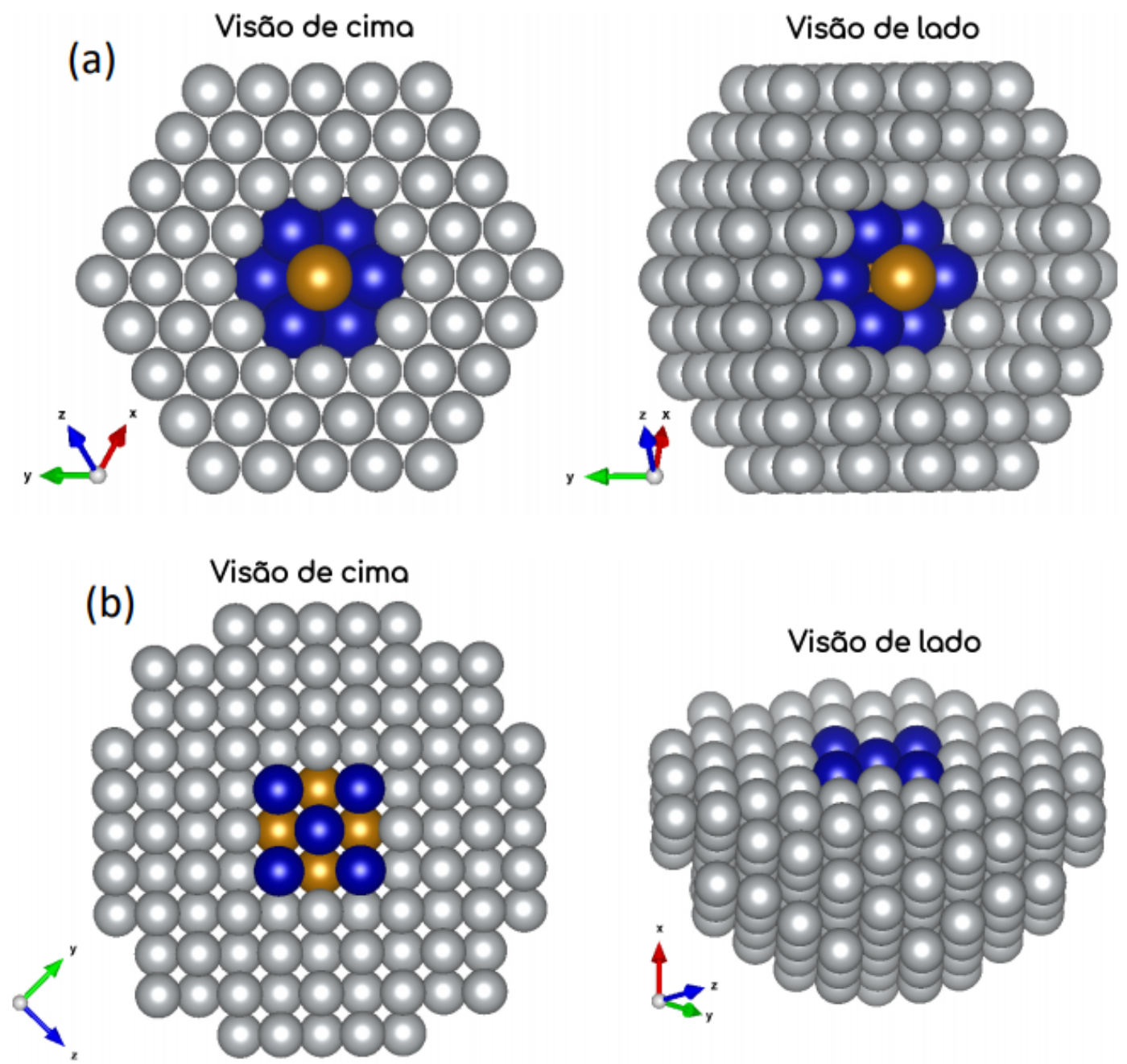

Figura 6.15: Representação esquemática de um exemplo de: (a) um cluster de Fecentrado contendo 15 átomos embebido em um meio VCA Fe ${ }_{50} \mathrm{Co}_{50}$ bcc; (b) um cluster de Co-centrado contendo 10 átomos embebido em uma superfície VCA $\mathrm{Fe}_{50} \mathrm{Co}_{50}(100)$ $b c c$. Esferas amarelas e azuis representam átomos de $\mathrm{Fe}$ e Co, respectivamente, enquanto as esferas cinzas representam os sítios de $\mathrm{VCA} \mathrm{Fe}_{50} \mathrm{Co}_{50}$ (8,5 elétrons de valência). A concentração de Fe (Co) nos clusters é: (a) 53\% (47\%); e (b) $50 \%$ (50\%). O número de átomos total incluindo os sítios VCA que contornam os clusters são: (a) 339; e (b) 293. Todos os átomos representados (incluindo o VCA circundante) foram considerados na obtenção de $\alpha_{t o t}$ no átomo central de (a) Fe; e (b) Co (de referência).

Os primeiros cálculos foram realizados em modelos bulk (B2-FeCo e VCA Fe ${ }_{50} \mathrm{Co}_{50}$ $b c c$ ). A partir das comparações com valores bem estabelecidos da literatura (Tabela 6.5), 
percebe-se que há uma considerável diferença entre os dampings totais calculados para a liga de $\mathrm{Fe}_{50} \mathrm{Co}_{50}$ na aproximação VCA, aleatória $\left(\alpha_{t o t}=3,7 \times 10^{-3}\right)$, e na estrutura B2, ordenada $\left(\bar{\alpha}_{t o t}=2,3 \times 10^{-3}\right)$. Além disso, nota-se a mesma discrepância quando se compara os valores de $\alpha_{t o t}$ entre o sistema de B2-FeCo e o bulk de Fe puro, bcc $\left(\alpha_{t o t}=\right.$ $\left.4,2 \times 10^{-3}\right)$. A fim de analisar a influência da composição de Co na liga, elaborou-se a Fig. 6.16. Nela, os valores de damping não-local $\left(\alpha_{i j}\right)$ entre pares de átomos Fe-Fe, Co-Co e Co-Fe nos bulks de B2-FeCo e Fe bcc são apresentados como função da distância interatômica. Já no Inset, os pontos representam a soma de todas as contribuições nãolocais entre Fe-Fe para uma dada distância.

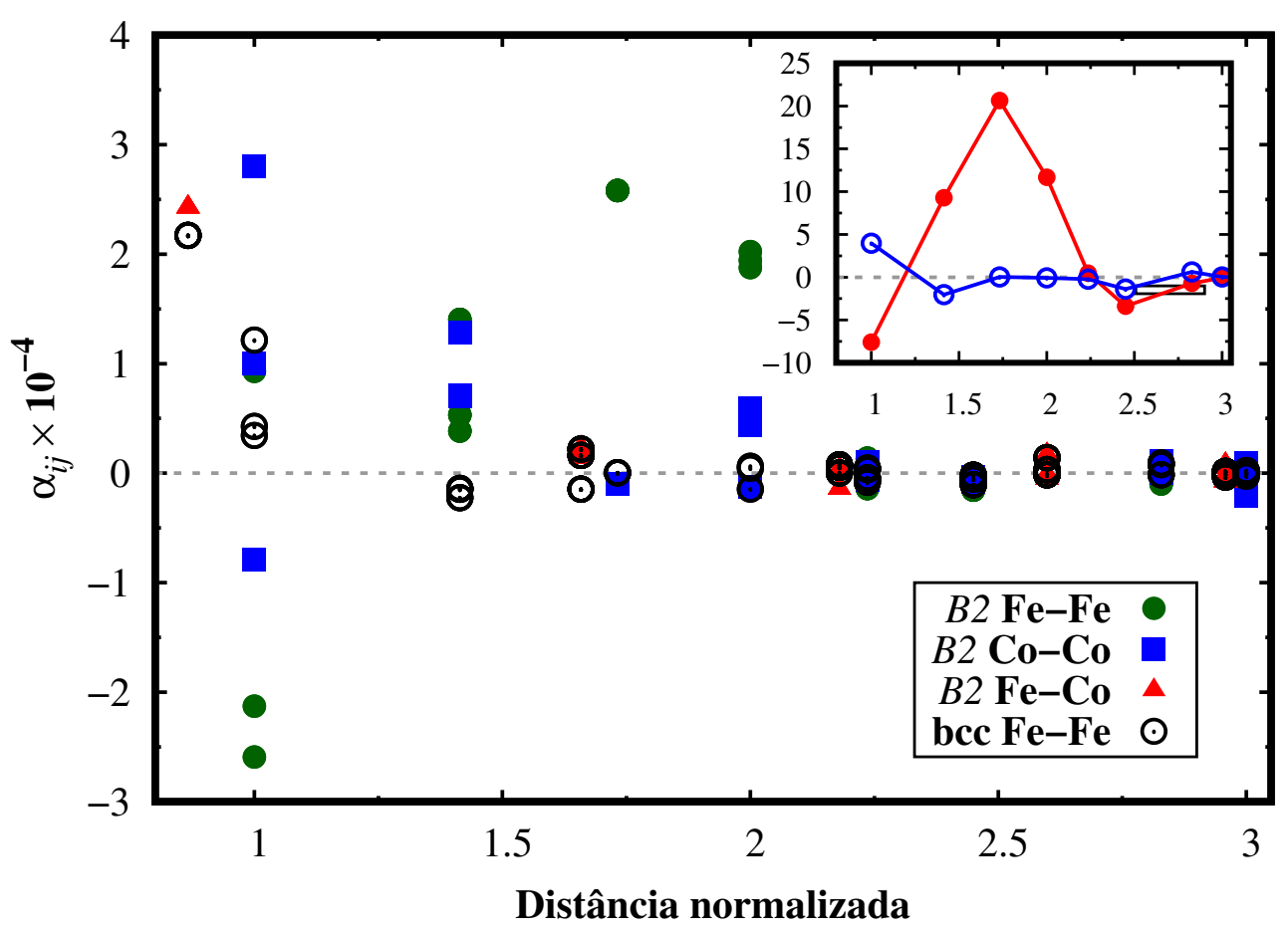

Figura 6.16: Damping não-local, $\alpha_{i j}$, nos bulks B2-FeCo e Fe bcc como função da distância interatômica normalizada (unidades de $a$ ). Inset: Contribuições não-locais para pares Fe-Fe somadas para cada distância normalizada pares $\mathrm{Fe}-\mathrm{Fe}$ para o Fe bulk bcc (pontos azuis) e para a liga $B 2-\mathrm{FeCo}$ (pontos vermelhos). Os valores de damping onsite para o $\mathrm{Fe}(\mathrm{Co})$ na estrutura B2-FeCo são $\alpha_{\text {onsite }}^{\mathrm{Fe}}=1,1 \times 10^{-3}\left(\alpha_{\text {onsite }}^{\mathrm{Co}}=0,8 \times 10^{-3}\right)$, e para o Fe bulk bcc é $\alpha_{\text {onsite }}^{\mathrm{Fe}}=1,6 \times 10^{-3}$. Para todos os resultados, considerou-se o eixo de magnetização como sendo o eixo- $z$ ([001]).

Como se pode notar, o damping não-local entre átomos Fe-Fe é distinto para as pequenas distâncias interatômicas, evidenciando a influência dos átomos de Co no entorno - no caso da liga B2-FeCo. Já para longas distâncias $\left(\frac{d}{a} \gtrsim 2,25\right)$, as contribuições de $\alpha_{i j}$ 
são similares nos dois sistemas, e também menos intensas $\left(\alpha_{i j} \rightarrow 0\right.$ para $\left.d \rightarrow \infty\right)$.

Voltando-se, agora, para a anisotropia de damping nestes casos $\left(\Delta \alpha_{t o t}\right)$, isto é, a variação percentual de $\alpha_{t o t}$ quando a direção principal de magnetização é modificado do eixo fácil para alguma outra direção. Assim, pode-se definir $\Delta \alpha_{t o t}{ }^{1}$ como:

$$
\Delta \alpha_{t o t}=\left(\frac{\alpha_{t o t}^{b}}{\alpha_{t o t}^{[010]}}-1\right) \times 100 \%
$$

onde $\alpha_{t o t}^{b}$ e $\alpha_{t o t}^{[010]}$ representam, respectivamente, o damping total quando a magnetização está paralela a uma direção $b$ qualquer, e ao eixo fácil $([010])^{2}$. Uma definição análoga também se aplica a $\Delta \alpha_{\text {onsite }}$. Investigou-se esta anisotropia em superfícies e em sistemas bulk com (e sem) distorções estruturais tetragonais ("bct-like"). Nossos cálculos para VCA $\mathrm{Fe}_{50} \mathrm{Co}_{50}$ bcc mostram um aumento de damping de $~ 13 \%$, ao mudar a direção da magnetização de [010] para [110]. A Tabela 6.8, mostra a variação obtida de $\alpha_{\text {tot }}$ para um sítio típico em diferentes sistemas para diferentes eixos de quantização de spin.

\footnotetext{
${ }^{1}$ Esta definição é diferente da definida na Ref. [255], na qual há uma referência à razão entre os valores máximo e mínimo de damping, isto é, $\frac{\alpha_{t o t}^{b}}{\alpha_{t o t}^{[010]}} \times 100 \%$.

${ }^{2}$ Para o estudo analisado, escolheu-se, na maioria das vezes, $b \equiv[110]$. Entretanto, nos casos de distorção tetragonal ("bct-like"), também analisou-se a anisotropia $\Delta \alpha_{\text {tot }}$ com $b \equiv[001]$.
} 
Tabela 6.8: Damping total $\left(\alpha_{t o t} \times 10^{-3}\right)$ de um átomo típico em cada sistema (bulk e superfície) para os eixos de quantização [010] e [110]; também demonstrado para o eixo [001]. Os casos bct são foram simulados considerando-se $\frac{c}{a}=1,15$.

\section{Bulks}

\begin{tabular}{|c|c|c|c|}
\hline Bulk & $\alpha_{t o t}[010]$ & $\alpha_{t o t}[110]$ & $\Delta \alpha_{t o t}$ \\
\hline $\mathrm{Fe} b c c$ & 4,18 & 4,31 & $+3,1 \%$ \\
\hline$B 2-\mathrm{FeCo} b c c$ & 2,28 & 2,44 & $+7,2 \%$ \\
\hline$B 2-\mathrm{FeCo} b c t$ & 7,76 & 8,85 & $+12,4 \%$ \\
\hline $\mathrm{VCA} \mathrm{Fe}_{50} \mathrm{Co}_{50} b c c$ & 3,70 & 4,18 & $+13,0 \%$ \\
\hline \multirow[t]{2}{*}{ VCA $\mathrm{Fe}_{50} \mathrm{Co}_{50} b c t$} & 4,69 & 5,10 & $+8,7 \%$ \\
\hline & $\alpha_{t o t}[010]$ & $\alpha_{t o t}[001]$ & $\Delta \alpha_{t o t}$ \\
\hline$\overline{B 2-\mathrm{FeCo} b c t}$ & 7,76 & 10,21 & $+24,1 \%$ \\
\hline VCA $\mathrm{Fe}_{50} \mathrm{Co}_{50} b c t$ & 4,69 & 5,75 & $+22,6 \%$ \\
\hline
\end{tabular}

\section{Superfícies}

\begin{tabular}{c|c|c|c}
\hline Superfície & $\alpha_{\text {tot }}[\mathbf{0 1 0}]$ & $\alpha_{\text {tot }}[\mathbf{1 1 0}]$ & $\Delta \alpha_{t o t}$ \\
\hline $\mathrm{Fe}(100) b c c$ & 3,49 & 3,47 & $-0,6 \%$ \\
VCA Fe$_{50} \mathrm{Co}_{50}(100) b c c$ & 7,00 & 14,17 & $+102,4 \%$ \\
VCA Fe $_{50} \mathrm{Co}_{50}(100) b c t$ & 15,20 & 14,80 & $-2,6 \%$ \\
\hline & $\alpha_{t o t}[\mathbf{0 1 0}]$ & $\alpha_{t o t}[\mathbf{0 0 1}]$ & $\Delta \alpha_{t o t}$ \\
\hline VCA Fe $_{50} \mathrm{Co}_{50}(100) b c t$ & 15,20 & 15,56 & $+2,4 \%$ \\
\hline
\end{tabular}

Como se pode notar, o menor damping é encontrado para o eixo de magnetização fácil, [010], que contém o maior momento orbital [120]. Para o bulk VCA Fe ${ }_{50} \mathrm{Co}_{50}$ bcc, além disso, obtivemos uma pequena variação de $\sim 2 \%$ para a contribuição no local $\left(\alpha_{\text {onsite }}^{[010]}=\right.$ $8,94 \times 10^{-4}$ e $\left.\alpha_{\text {onsite }}^{[110]}=8,76 \times 10^{-4}\right)$, o que implica que a anisotropia vem principalmente das contribuições não locais, em particular dos vizinhos mais próximos, como se pode ver na Fig. 6.17 (mais especificamente nos segundos vizinhos). Para comparação, no caso do Fe bcc $\Delta \alpha_{\text {tot }} \sim 3 \%$ (com $\Delta \alpha_{\text {onsite }} \sim 0,4 \%$ ), o que corrobora o resultado já apresentado na literatura [66] de uma pequena anisotropia de damping do Fe bcc à temperatura ambiente. Além disso, o aumento calculado em $\alpha_{t o t}$ quando se muda da estrutura bcc do $\mathrm{Fe}_{50} \mathrm{Co}_{50}$ $\left(\frac{c}{a}=1\right)$ para a estrutura bct $\left(\frac{c}{a}=1,15\right)$ é qualitativamente consistente com o reportado por Mandal et al. [243], que calcularam um aumento de $\alpha_{t o t}=6,6 \times 10^{-3}\left(\mathrm{Fe}_{50} \mathrm{Co}_{50} b c c\right)$ para $\alpha_{t o t}=17,8 \times 10^{-3}\left(\mathrm{Fe}_{50} \mathrm{Co}_{50} b c t, \operatorname{com} \frac{c}{a}=1,33\right)$. 


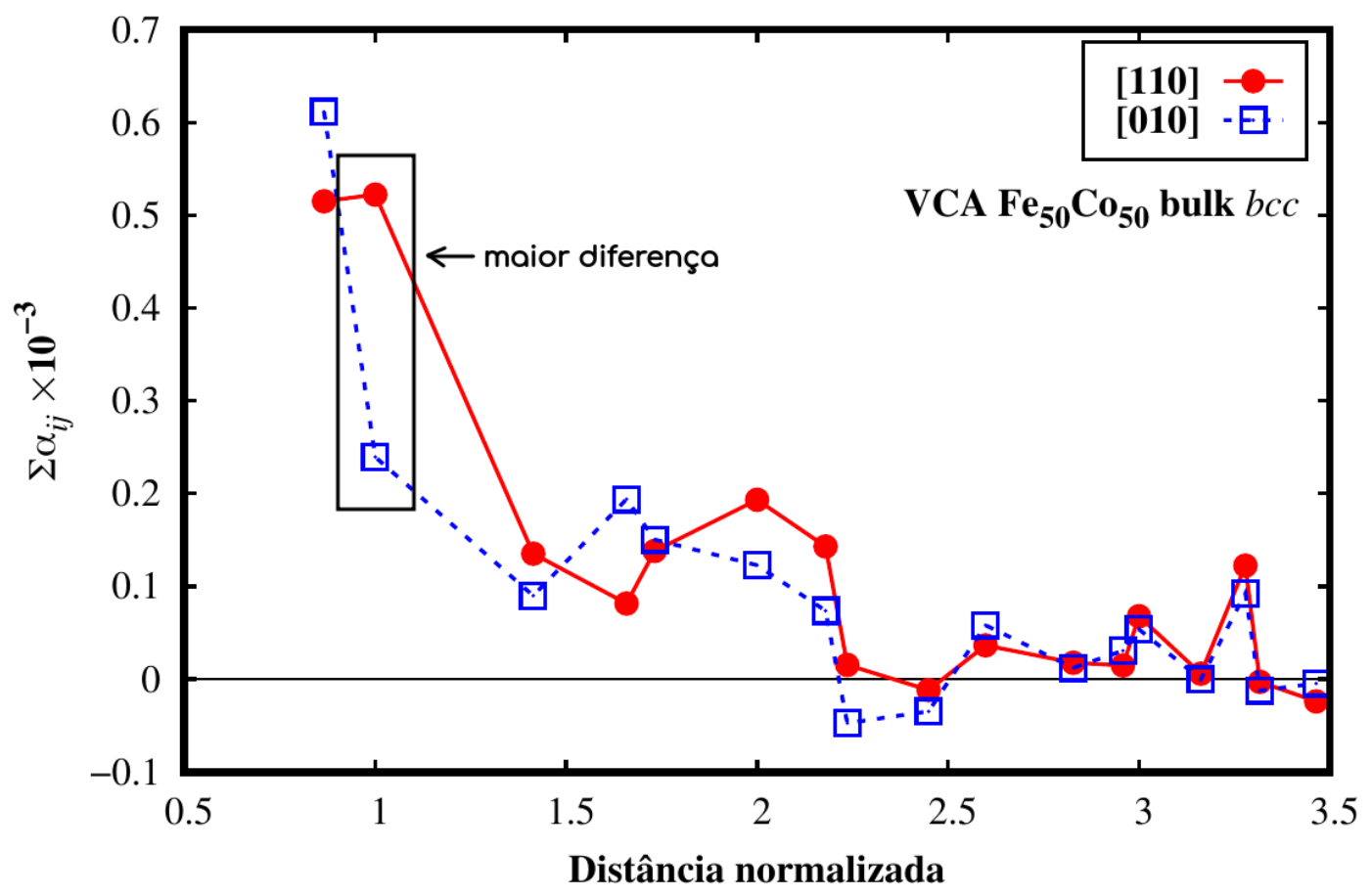

Figura 6.17: Soma das contribuições não-locais de damping $\alpha_{i j}\left(\times 10^{-3}\right)$ para cada distância normalizada determinada (em função do parâmetro de rede a) do bulk VCA $\mathrm{Fe}_{50} \mathrm{Co}_{50}$ bcc nas direções de magnetização: (i) [110] (em vermelho); e (ii) [010] (em azul).

Também inspecionamos a influência da inomogeneidade química na anisotropia, considerando, como um primeiro caso natural, a liga $B 2-\mathrm{FeCo}$, onde o amortecimento médio ponderado (Eq. 6.19) foi usado. O valor de $\Delta \alpha_{t o t}$ tanto para o B2-FeCo bcc $(\sim 7 \%)$ quanto para o VCA $\mathrm{Fe}_{50} \mathrm{Co}_{50} b c c(\sim 13 \%)$ são de magnitudes semelhantes. Tanto a estrutura B2 quanto os cálculos VCA levam a anisotropias de damping que são significativamente menores do que o observado nos experimentos, e parece provável que a presença de desordem na composição e/ou propriedades estruturais da liga Fe/Co são de grande importância para produzirem efeitos consideráveis na anisotropia de damping. Discutimos essa possibilidade logo a seguir.

Um aspecto importante para o aumento do damping é o papel de possíveis distorções locais na rede, como sugerido na Ref. [255]. Analisamos essa possibilidade considerando um caso hipotético de uma grande distorção no eixo $c$ do $B 2$-FeCo ordenado, de $15 \%$ (ou $\left.\frac{c}{a}=1,15\right)$. Neste caso, tal como apresentado na Tabela $\mathbf{6 . 8}$, encontrou-se que o maior valor de $\Delta \bar{\alpha}_{t o t}$ é de $\sim 24 \%$, considerando-se a diferença de $\alpha_{t o t}$ entre os eixos [010] e [001] (agora não mais isotrópico, como no caso $b c c$ ). Isso confirma que, de fato, as distorções do tipo bct atuam em favor do aumento de $\Delta \alpha_{t o t}$ (e, portanto, da razão entre os valores máximos e mínimos de damping), embora os dados apresentados ainda não sejam suficientes para 
explicar o gigantesco aumento em $\alpha_{\text {tot }}$ observado experimentalmente [255].

No entanto, no caso de uma liga, as distorções locais sugeridas em [255] são mais prováveis de ocorrer de uma maneira heterogênea [259], isto é, com diferentes distribuições espaciais (arranjos) de Fe/Co ao longo da amostra. A fim de verificar teoricamente a influência desta heterogeneidade, investigamos clusters contendo diferentes configurações atômicas de $\mathrm{Fe} / \mathrm{Co}$, embebidos em uma matriz VCA de $\mathrm{Fe}_{50} \mathrm{Co}_{50}$ (com o parâmetro de rede do Fe bulk bcc); distorções locais nestes clusters também foram consideradas, seguindo a relação $\frac{c}{a}=1,15$. Ao todo, 10 configurações distintas foram calculadas (um exemplo é esquematizado na Fig. 6.15): 5 com um Fe como sítio de referência (Fe-centrado), e 5 com um Co como sítio de referência (Co-centrado) ${ }^{1}$. Os clusters nos sistemas bulk foram construídos de forma a incluir os vizinhos com uma distância até $1 a$ em torno de um sítio de referência, respeitando a simetria do sistema. Sendo assim, os clusters embebidos em um substrato bcc (bulk) contam com um total de 15 átomos, resultando em uma concentração de Fe/Co de 53\%/47\%, ou vice-versa. Em todos os casos, os clusters de FeCo foram tratados de forma autoconsistente, sendo que os parâmetros de potencial dos sítios VCA restantes (no entorno) foram fixados naqueles calculados para o $\mathrm{Fe}_{50} \mathrm{Co}_{50}$ bulk. Além disso, visando evitar qualquer efeito de superfície indesejado, o átomo de referência foi posicionado exatamente no centro da matriz VCA no espaço-real. Para os clusters no VCA $\mathrm{Fe}_{50} \mathrm{Co}_{50}$ bulk bcc, os valores de $\bar{\alpha}_{t o t}$ foram, então, calculados como a soma das contribuições onsite e não-locais com um átomo central específico (Fe ou Co) - sendo tomada, então, a média dos clusters Fe- e Co-centrados. Os resultados obtidos de $\bar{\alpha}_{t o t}$ para ambos os tipos de cluster, tanto na estrutura bcc quanto aqueles com distorção bct, são apresentados nas Tabelas $\mathbf{6 . 9}$ e $\mathbf{6 . 1 0}$, respectivamente.

Tabela 6.9: Anisotropia e damping total $\left(\times 10^{-3}\right)$ de todos os clusters Fe- e Co-centrados $b c c$ estudados, considerando os eixos de magnetização [010] e [110]. Os clusters apresentam 15 átomos de $\mathrm{FeCo}$, embebidos em um meio VCA de $\mathrm{Fe}_{50} \mathrm{Co}_{50}$ bulk bcc. Os valores de $\alpha_{\text {tot }}$ levam em consideração no somatório os sítios VCA ao redor.

\begin{tabular}{|c|c|c|c|c|c|c|c|}
\hline \multicolumn{4}{|c|}{ Co-centrados } & \multicolumn{4}{|c|}{ Fe-centrados } \\
\hline$\#$ & $\alpha_{t o t}[010]$ & $\alpha_{t o t}[\mathbf{1 1 0}]$ & $\left|\Delta \alpha_{t o t}\right|$ & $\#$ & $\alpha_{t o t}[010]$ & $\alpha_{t o t}[\mathbf{1 1 0}]$ & $\left|\Delta \alpha_{t o t}\right|$ \\
\hline 1 & 10,11 & 9,65 & $4,8 \%$ & 1 & 2,68 & 2,03 & $32,0 \%$ \\
\hline 2 & 8,09 & 6,96 & $16,2 \%$ & 2 & 2,49 & 2,05 & $21,5 \%$ \\
\hline 3 & 7,81 & 7,02 & $11,3 \%$ & 3 & 2,56 & 1,86 & $37,6 \%$ \\
\hline 4 & 7,11 & 7,02 & $1,3 \%$ & 4 & 2,45 & 1,79 & $36,9 \%$ \\
\hline 5 & 7,48 & 6,88 & $8,7 \%$ & 5 & 2,76 & 2,01 & $37,3 \%$ \\
\hline $\bar{\alpha}_{t o t}$ & 8,12 & 7,51 & $8,1 \%$ & $\bar{\alpha}_{t o t}$ & 2,59 & 1,95 & $32,8 \%$ \\
\hline
\end{tabular}

\footnotetext{
${ }^{1}$ Nota-se que o número de clusters considerado é limitado na média estatística.
} 
Tabela 6.10: Anisotropia e damping total $\left(\times 10^{-3}\right)$ de todos os clusters Fe- e Cocentrados estudados, considerando os eixos de magnetização [010] e [110] e uma distorção do tipo bct com $\frac{c}{a}=1,15$. Os clusters apresentam 15 átomos de FeCo, embebidos em um meio VCA de $\mathrm{Fe}_{50} \mathrm{Co}_{50}$ bulk bcc. Os valores de $\alpha_{t o t}$ levam em consideração no somatório os sítios VCA ao redor.

\begin{tabular}{|c|c|c|c|c|c|c|c|}
\hline \multicolumn{4}{|c|}{ Co-centrados } & \multicolumn{4}{|c|}{ Fe-centrados } \\
\hline$\#$ & $\alpha_{t o t}[010]$ & $\alpha_{t o t}[\mathbf{1 1 0}]$ & $\left|\Delta \alpha_{t o t}\right|$ & $\#$ & $\alpha_{t o t}[010]$ & $\alpha_{t o t}[\mathbf{1 1 0}]$ & $\left|\Delta \alpha_{t o t}\right|$ \\
\hline 1 & 5,85 & 4,37 & $33,9 \%$ & 1 & 2,36 & 1,39 & $69,8 \%$ \\
\hline 2 & 5,95 & 4,21 & $41,3 \%$ & 2 & 2,27 & 1,32 & $72,0 \%$ \\
\hline 3 & 5,88 & 4,35 & $35,2 \%$ & 3 & 2,22 & 1,26 & $76,2 \%$ \\
\hline 4 & 5,90 & 4,41 & $33,8 \%$ & 4 & 2,25 & 1,26 & $78,6 \%$ \\
\hline 5 & 5,86 & 4,34 & $35,0 \%$ & 5 & 2,42 & 1,38 & $75,4 \%$ \\
\hline $\bar{\alpha}_{t o t}$ & 5,89 & 4,34 & $35,7 \%$ & $\bar{\alpha}_{t o t}$ & 2,30 & 1,32 & $74,2 \%$ \\
\hline
\end{tabular}

A comparação entre os resultados das Tabelas 6.9 e $\mathbf{6 . 1 0}$ revela que o valor absoluto de $\alpha_{t o t}\left(\right.$ e $\left.\bar{\alpha}_{t o t}\right)$ diminui para os clusters com distorções bct, embora a anisotropia de damping seja maior. Em todos os casos, clusters Fe-centrados apresentaram valores maiores de $\Delta \alpha_{\text {tot }}$. Na média, portanto, os clusters Fe-centrados exibiram uma anisotropia de damping de $\sim 33 \%$ para os não-distorcidos ( $\sim 74 \%$ para os distorcidos), e os clusters Co-centrados uma anisotropia de $\sim 8 \%$ para os não-distorcidos ( $\sim 36 \%$ para os distorcidos). Embora estes resultados demonstrem a importância das distorções locais tipo-bct e das contribuições não-locais à anisotropia de damping, ainda não são suficientes para reproduzir a enorme razão de $\sim 400 \%$ entre os valores máximo e mínimo de $\alpha_{\text {tot }}$ [255].

Isso indica que a existência de um aumento tão pronunciado do damping entre as direções de magnetização [010] e [110] observado na referência [255] deve ter outras origens. Tomando como base o fato de os autores realizarem experimentos em filmes de $\mathrm{Fe}_{50} \mathrm{Co}_{50}$, passamos a analisar os efeitos de interface, presentes em filmes finos, bordas de grãos, falhas de empilhamento, superfícies de materiais, e materiais em geral. Essas interfaces podem influenciar as propriedades observadas, e, portanto, considerou-se estes efeitos explicitamente nos cálculos. Como um modelo, adotou-se uma superfície livre de $\mathrm{Fe}_{50} \mathrm{Co}_{50}(100)$ como um caso extremo. Assim, realizamos uma série de cálculos para o $\mathrm{Fe}_{50} \mathrm{Co}_{50}(100)$, em princípio utilizando a aproximação VCA. De forma análoga aos sistemas bulk, encontrou-se que a anisotropia de damping devido à contribuição onsite é relativamente pequena $\left(\Delta \alpha_{\text {onsite }} \sim 18 \%\right)$. No entanto, a quebra de simetria de inversão neste caso resulta em um elevado aumento de $\Delta \alpha_{\text {tot }}$ advindo das contribuições não-locais, particularmente de segundos-vizinhos. A anisotropia total neste caso foi $\sim 100 \%$ (que corresponde a uma razão entre o máximo e o mínimo valor de damping de $\sim 200 \%$ ). Um 
compilado dos resultados teóricos mais relevantes é mostrado na Figura 6.18. Nota-se, pela figura, que a variação de $\alpha_{t o t}$ para a superfície VCA $\mathrm{Fe}_{50} \mathrm{Co}_{50}(100)$ é não-linear, e os menores (maiores) valores de damping foram obtidos para o eixo de magnetização [010], $\theta=90^{\circ}\left([110], \theta=135^{\circ}\right)$.

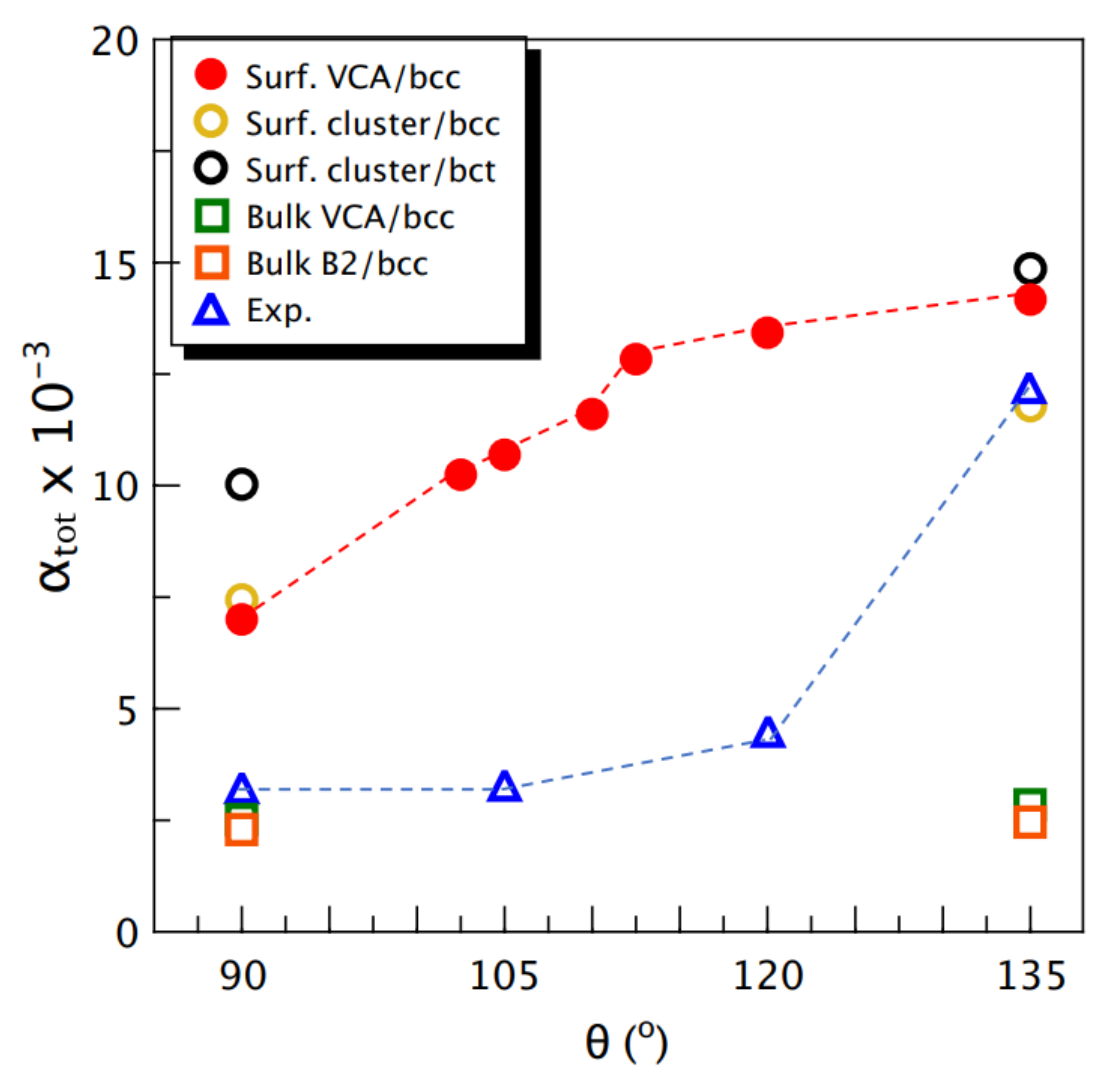

Figura 6.18: Damping total em função de $\theta$, o ângulo entre a direção de magnetização e o eixo [001]. Observe que $\theta=90^{\circ}$ corresponde a [010] e $\theta=135^{\circ}$ ao eixo de magnetização [110]. Círculos: (vermelho, preenchido) VCA Fe ${ }_{50} \mathrm{Co}_{50}(100) b c c$; (amarelo, vazio) média dos 32 clusters (16 Fe-centrados e 16 Co-centrados), com estrutura bcc nas camadas de superfície e incorporadas em um meio VCA (Fig. 6.15); (preto, vazio) cálculos similares, mas com uma distorção local tipo-bct da rede (ver texto). Quadrados: (verde, vazio) média para o bulk VCA Fe ${ }_{50} \mathrm{Co}_{50}$ bcc; e (laranja, vazio) bulk B2-FeCo. Triângulos: (azul, vazio) dados experimentais [255] para um filme de $\mathrm{Fe}_{50} \mathrm{Co}_{50} / \mathrm{Pt}$ com espessura de $10 \mathrm{~nm}$.

Pelos dados apresentados na Tabela 6.8, a superfície de Fe bcc exibe uma anisotropia desprezível de damping ( 1\%). De acordo com a fórmula simplificada de Kamberský (Eq. 6.13), três fatores podem alterar o valor de $\alpha_{t o t}$ quando há a mudança de direção de magnetização: $(i)$ o strength do acoplamento spin-órbita, $\xi$; (ii) o valor do momento magnético de spin, $m_{S}$; e (iii) a LDOS no nível de Fermi, $n\left(E_{F}\right)\left(\Delta \alpha_{t o t} \propto \Delta n\left(E_{F}\right)\right)$. Nos dois primeiros casos, não se encontrou variação significativa $\left(\xi_{\text {Co }}=71,0 \mathrm{meV}\right.$ e $\xi_{\mathrm{Fe}}=53,5 \mathrm{meV}$, em qualquer eixo). Portanto, diferentemente do que propõem os autores 
da referência $[255]^{1}$, pode-se relacionar a anisotropia de damping à variação de $n\left(E_{F}\right)$. A fim de demonstrar a existência de uma anisotropia de $n\left(E_{F}\right)$, calculou-se a diferença da LDOS: para um sítio típico na primeira camada da superfície VCA Fe ${ }_{50} \mathrm{Co}_{50}(100)$; total do sistema (soma de todas as LDOS); e para um sítio típico na primeira camada da superfície de $\mathrm{Fe} b c c$, em ambos os canais de spin. Os gráficos resultantes são mostrados na Figura 6.19.

\footnotetext{
${ }^{1} \mathrm{Em}[255]$, os autores argumentam que não foi encontrada uma anisotropia em $n\left(E_{F}\right)$ para diferentes orientações de magnetização. Contudo, os cálculos ab-initio realizados nesta referência centraram-se na investigação do bulk de $\mathrm{Fe}_{50} \mathrm{Co}_{50}$, sendo considerada apenas a densidade de estados total do sistema.
} 


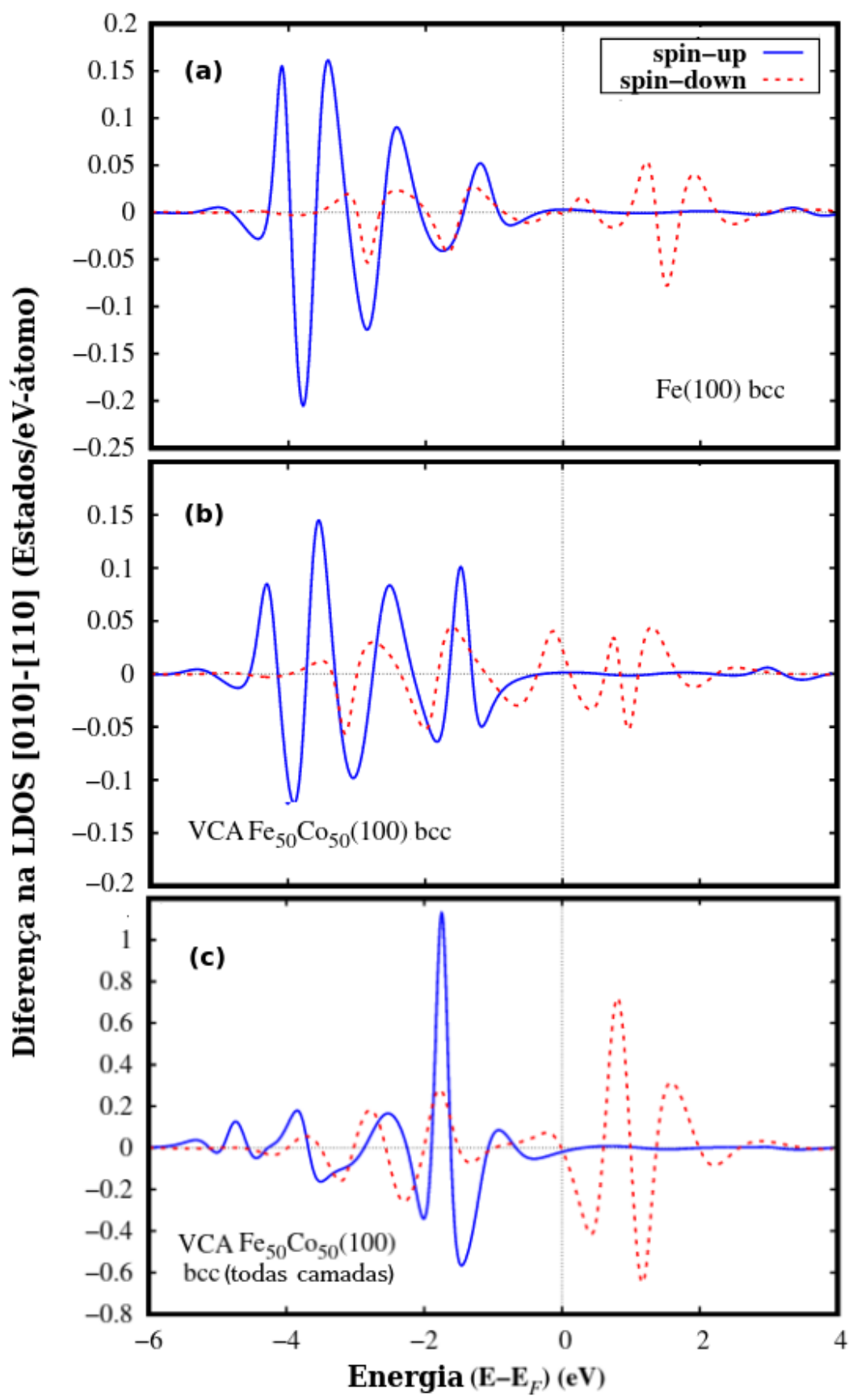

Figura 6.19: Diferença na LDOS (por átomo), $\Delta n(E)$, entre as direções de magnetização [010] e [110], para ambos os canais de spin em(na): (a) camada mais externa, próxima ao vácuo, em uma superfície de Fe $b c c ;(b)$ camada mais externa, próxima ao vácuo, em uma superfície VCA $\mathrm{Fe}_{50} \mathrm{Co}_{50}(100)$ bcc; e $(c)$ todas as camadas somadas no caso VCA $\mathrm{Fe}_{50} \mathrm{Co}_{50}(100)$ bcc.

Vê-se, portanto, que, embora a quantidade $\Delta n(E)$ apresente algumas oscilações para $E \neq E_{F}$, esta só exibe um valor não-desprezível em $E_{F}$ no canal de spin-down para a superfície $\mathrm{VCA} \mathrm{Fe}_{50} \mathrm{Co}_{50}(100)$, quando se considera um sítio típico na camada mais externa (próxima ao vácuo). Portanto, o surgimento de uma elevada anisotropia de damping no 
sistema VCA $\mathrm{Fe}_{50} \mathrm{Co}_{50}(100)$ está, de fato, relacionado à diferença na densidade local de estados no nível de Fermi, que corrobora com os resultados anteriores. Interessante também notar que, ao se considerar a LDOS total da superfície VCA Fe ${ }_{50} \mathrm{Co}_{50}(100)$, $\Delta n\left(E_{F}\right) \rightarrow 0$ (Fig. 6.19(c)), tal como foi verificado em [255].

Além da relação $\Delta \alpha_{t o t} \propto \Delta n\left(E_{F}\right)$, uma importante indicação da Fig. 6.19 é que o efeito vem sobretudo da camada mais externa da superfície - justamente a mais influenciada pelos efeitos da quebra de simetria de inversão. Essa observação é demonstrada por uma inspeção camada-por-camada da contribuição intralayer ao damping, cujos valores estão na Tabela $\mathbf{6 . 1 1}$.

Tabela 6.11: Damping intralayer total $\left(\alpha_{t o t} \times 10^{-3}\right)$ e anisotropia $\Delta \alpha_{t}$ (Eq. 6.27), de um átomo típico (cálculos com aproximação VCA) em cada camada da superfície $\mathrm{Fe}_{50} \mathrm{Co}_{50}(100)$ bcc, para as direções de magnetização [010] e [110]. Em cada linha, a soma de todos $\alpha_{i j}$ é considerada. Camada "1" denota a mais externa, próxima à região de vácuo, e as camadas $2-5$ denotam as adjacentes, e mais profundas.

\begin{tabular}{c|c|c|c}
\hline Camada & $\alpha_{\text {tot }}[\mathbf{0 1 0}]$ & $\alpha_{\text {tot }}[\mathbf{1 1 0}]$ & $\Delta \alpha_{t o t}$ \\
\hline 1 & 7,00 & 14,17 & $+102,4 \%$ \\
2 & 1,28 & 1,16 & $-9,4 \%$ \\
3 & 2,83 & 3,30 & $+16,6 \%$ \\
4 & 2,18 & 1,99 & $-8,7 \%$ \\
5 & 2,54 & 2,53 & $-0,4 \%$ \\
\hline Total & - & - & $+100,5 \%$ \\
\hline Camadas 2-5 & - & - & $-1,9 \%$ \\
\hline
\end{tabular}

As camadas mais profundas da superfície exibem um comportamento quase oscilatório de $\Delta \alpha_{t o t}$, semelhante à mencionada na referência [67], e às oscilações de Friedel usualmente obtidas para os momentos magnéticos na superfície. Como esperado pelos resultados da LDOS, essas camadas mais profundas do sistema sofrem menos a influência da quebra de simetria de inversão. No entanto, a elevada anisotropia que surge da camada mais externa, como um efeito de superfície, concorda com o observado por Chen et al. [247] para o $\mathrm{Fe} / \mathrm{GaAs}(001)$, em que a anisotropia de damping desaparece com o aumento da espessura do filme de Fe (ou seja, $\alpha_{t o t}$ se torna cada vez mais anisotrópico quanto mais fino é o filme).

Como uma última etapa de investigação, prosseguimos com o estudo do impacto das distorções tipo-bct na superfície. Para isso, inicialmente foram construídos 32 diferentes clusters contendo 10 átomos $^{1}$, todos com a estrutura $b c c$, embutidos na superfície VCA $\mathrm{Fe}_{50} \mathrm{Co}_{50}(100)$ (Fig. 6.15(b)), sendo 16 Fe-centrados e 16 Co-centrados. Neste caso,

\footnotetext{
${ }^{1} \mathrm{Na}$ distância máxima de $1 a$ em torno de um sítio de referência.
} 
foi possível equilibrar uma concentração Fe/Co de exatamente 50\%/50\%. Alterando a direção de magnetização, encontrou-se uma variação no damping de $\alpha_{t o t}^{[010]}=7,44 \times 10^{-3}$ para $\alpha_{t o t}^{[110]}=11,79 \times 10^{-3}\left(\right.$ ou $\left.\Delta \alpha_{t o t} \sim 58 \%^{1}\right)$. Um resumo dos resultados obtidos para os 32 clusters não-distorcidos na superfície é apresentado na Fig. 6.20: Co-centrados na Fig. 6.20(a) e Fe-centrados na Fig. 6.20(b).

Uma elevada diferença dos valores $\alpha_{\text {tot }}$ é verificada de cluster para cluster, dependendo da distribuição espacial das espécies atômicas. É claro, pelos gráficos da Fig. 6.20, que $\alpha_{t o t}$ é maior quando há mais átomos de Fe na camada mais externa da superfície, cercando o sítio de referência. Essa correlação pode ser vista pelos números em parêntesis acima de cada símbolo azul. Além disso, pela análise da mesma figura, o damping de ambos clusters Fe- e Co-centrados é maior quando o eixo principal de magnetização é paralelo à direção [110]. Já no Inset da Fig. 6.20, nota-se uma forte correlação entre a contribuição $\alpha_{\text {onsite }}$ e a LDOS no nível de Fermi do sítio central. Como uma observação geral, a comparação entre $\alpha_{\text {tot }}$ e $\alpha_{\text {onsite }}$ permite concluir que a vizinhança influencia não apenas a estrutura eletrônica local do átomo de referência, mas também provoca uma significativa mudança nos termos não-locais para o damping, $\alpha_{i j}$, particularmente para a direção [110].

Partindo para as distorções do tipo bct na superfície, considerou-se agora $\frac{c}{a}=1,09{ }^{2}$. Convergida a superfície bct na aproximação VCA, encontrou-se uma variação no damping $\operatorname{de} \alpha_{t o t}^{[010]}=8,94 \times 10^{-3}$ para $\alpha_{t o t}^{[110]}=15,68 \times 10^{-3}$ (ou $\Delta \alpha_{t o t} \sim 75 \%$ ). Aqui, novamente, um dos efeitos da distorção tipo-bct é o aumento do damping absoluto, nas duas direções de magnetização. Isso ocorre, essencialmente, por uma amplificação na contribuição onsite, que passa de $\alpha_{\text {onsite }}^{[010]}=7,4 \times 10^{-3}$ (não-distorcido) para $\alpha_{\text {onsite }}^{[010]}=9,5 \times 10^{-3}$ (distorcido), e de $\alpha_{\text {onsite }}^{[110]}=8,7 \times 10^{-3}$ (não-distorcido) para $\alpha_{\text {onsite }}^{[110]}=11,7 \times 10^{-3}$ (distorcido), permanecendo as contribuições não-locais bastante similares em ambos os casos. Finalmente, utilizando os mesmos 32 clusters, mas considerando a mesma distorção $\frac{c}{a}=1,09$ nos primeiros vizinhos em torno do átomo central, encontrou-se uma variação no damping de $\alpha_{t o t}^{[010]}=$ $10,03 \times 10^{-3}$ para $\alpha_{t o t}^{[110]}=14,86 \times 10^{-3}\left(\Delta \alpha_{t o t} \sim 48 \%\right)$. Um resumo dos principais resultados de anisotropia de damping obtidos para estes e outros sistemas analisados está na Tabela 6.12 .

\footnotetext{
${ }^{1}$ Destaca-se, aqui, que a quantidade de clusters considerados é limitada na estatística.

${ }^{2}$ A porcentagem de distorção para os clusters na superfície, de $9 \%$, foi menor que a assumida para o bulk, de 15\%, por limitação do método. Para relaxações maiores que $9 \%$ na superfície, a matriz de estrutura não podia ser satisfatoriamente calculada.
} 

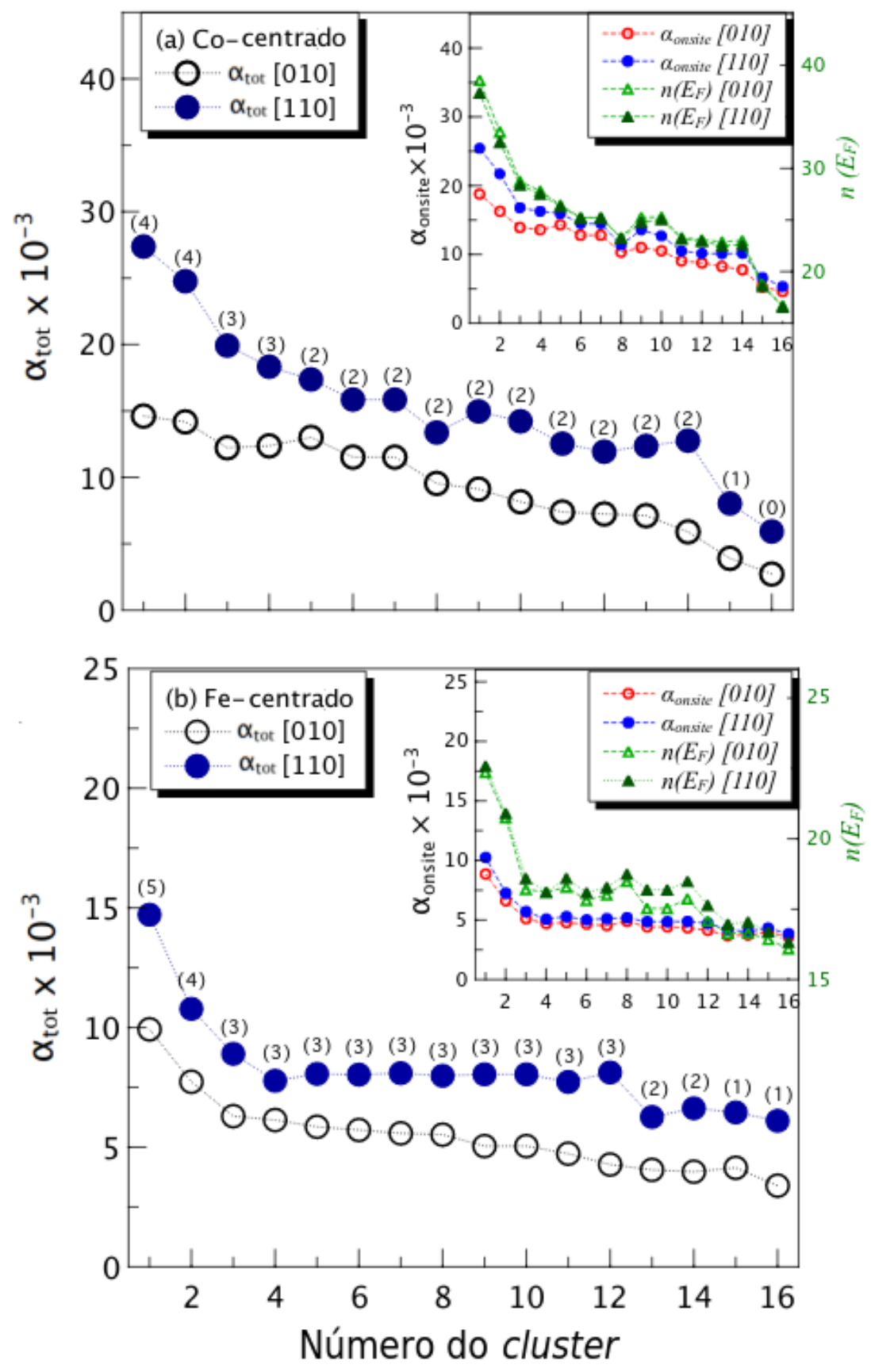

Figura 6.20: Damping para as direções de magnetização [010] (círculos pretos vazios) e [110] (círculos azul-escuro preenchidos) para diferentes tipos de clusters $\mathrm{Fe}_{50} \mathrm{Co}_{50}$ bcc contendo 10 átomos, embutidos em um substrato $\mathrm{VCA} \mathrm{Fe}_{50} \mathrm{Co}_{50}(100)$ bcc e sem qualquer distorção ao redor do átomo de referência (para o qual $\alpha_{t o t}$ e $\alpha_{\text {onsite }}$ são mostrados). Clusters do tipo (a) Co-centrados e (b) Fe-centrados. As quantidades de átomos de Fe na camada mais externa (próxima ao vácuo) são indicadas pelos números em parêntesis, e os resultados foram ordenados de forma que os maiores valores de damping aparecessem mais à esquerda. Insets: $\alpha_{\text {onsite }}$ para as orientações de magnetização [010] (círculos vermelhos vazios) e [110] (círculos azuis preenchidos), e a sua correspondente densidade local de estados no nível de Fermi, $n\left(E_{F}\right)$ (triângulos verdes vazios e preenchidos), no átomo central (localizado na camada mais externa) para ambos os tipos de clusters. 
Tabela 6.12: Resumo dos principais resultados de anisotropia de damping obtidos nos sistemas $\mathrm{Fe}_{50} \mathrm{Co}_{50}$ : liga pura ordenada (B2); liga pura aleatória bulk (VCA); bulk bcc com ordenamento de curto-alcance (OCA), isto é, com clusters embebidos (veja a Tabela 6.9); bulk com clusters distorcidos (tipo-bct) inseridos (Tabela 6.10); e cálculos de superfície, no modo pristino (puro) e com clusters explícitos embebidos (superfície + distorção). A razão máxima-mínima do damping, de acordo com a referência [255], é dada por: $\frac{\alpha_{t o t}^{[110]}}{\alpha_{t o t}^{[010]}} \times 100 \%$.

\begin{tabular}{c|c|c}
\hline Estrutura & $\Delta \alpha_{\text {tot }}$ & Razão máx-min \\
\hline Liga ordenada bcc & $7,2 \%$ & $107,2 \%$ \\
Liga ordenada bct & $24,1 \%$ & $124,1 \%$ \\
Liga aleatória bcc & $13 \%$ & $113 \%$ \\
Liga aleatória bct & $22,6 \%$ & $122,6 \%$ \\
Liga aleatória + OCA & $14,9 \%$ & $114,9 \%$ \\
Liga aleatória + OCA + Distorção & $47,2 \%$ & $147,2 \%$ \\
Superfície (camada externa) & $102,4 \%$ & $202,4 \%$ \\
Superfície (camada ext.) + Distorção & $75,4 \%$ & $175,4 \%$ \\
10-nm Co ${ }_{50} \mathrm{Fe}_{50} / \mathrm{Pt}[255]$ (exp.) & $281,3 \%$ & $381,3 \%$ \\
\hline \multicolumn{2}{|c|}{}
\end{tabular}




\section{Capítulo 7}

\section{Conclusões}

Neste trabalho, investigamos as propriedades eletrônicas e magnéticas, bem como a dinâmica de spins, de diversos sistemas bulk e superfícies. Estes estudos foram pautados em métodos que são, em conjunto e atualmente, o estado-da-arte na investigação do magnetismo em escala nanométrica: o RS-LMTO-ASA (cálculo da estrutura eletrônica no espaço-real), e o UppASD (cálculo da dinâmica de spins baseada no formalismo LLG).

Embora parte dos resultados tenha focado em casos concretos, como as superfícies de $\mathrm{Pd} / \mathrm{Fe} / \mathrm{Ir}(111), \mathrm{Pd} / \mathrm{Co} / \operatorname{Ir}(111), \mathrm{Fe}_{50} \mathrm{Co}_{50}(100)$, entre outras, um importante avanço deste trabalho foi o desenvolvimento teórico e/ou a implementação computacional de parâmetros como: o damping no espaço-real, a carga topológica $q$, a interação de DM como função da energia, e um método para se considerar defeitos em superfícies no contexto do UppASD. Cada um destes desenvolvimentos exigiu uma quantidade relevante de testes para certificar sua usabilidade em novos problemas; em particular, os testes relativos à implementação do damping são apresentados de forma detalhada, explorando os seus mais variados aspectos. Neste sentido, cabe também ressaltar que tanto o cálculo de damping de maneira local (por exemplo, em sistemas nanoestruturados), quanto o cálculo de dinâmica de spins considerando defeitos em superfícies são - no melhor conhecimento da literatura - inéditos, e abrem novos caminhos para uma compreensão mais aprofundada do magnetismo em sistemas heterogêneos e/ou contendo impurezas.

Dois dos sistemas estudados foram as multicamadas de Pd/Fe/Ir(111) e Pd/Co/Ir(111), para os quais o interesse era investigar as origens de uma rede de skyrmions na primeira. No caso do sistema $\mathrm{Pd} / \mathrm{Fe} / \mathrm{Ir}(111)$, obteve-se que o estado fundamental é caracterizado por um arranjo não-colinear de spins, no qual ambas as interações isotrópica (de troca) e anisotrópica (de DM) apresentam participações igualmente importantes. De fato, a partir 
dos cálculos com o pacote UppASD, verificamos que o estado fundamental da camada de Fe é constituído por espirais de spin com comprimento de onda $\lambda \sim 10,7 \mathrm{~nm}$, em boa concordância com trabalhos experimentais e teóricos previamente publicados. Encontrou-se que uma das principais origens desse estado não-colinear de menor energia é a significativa frustração magnética induzida pela competição FM-AFM entre as interações de troca de pares de átomos Fe-Fe. Dentre elas, podemos destacar o acoplamento entre terceiros vizinhos ( $\mathrm{a} \sim 5,43 \AA$ de distância $)$, negativo e com magnitude de $\sim 17 \%$ da interação entre primeiros vizinhos. Como foi demonstrado por meio de cálculos não-colineares sem considerar o efeito do acoplamento spin-órbita, essa frustração magnética não é suficiente, sozinha, para conduzir o sistema $\mathrm{Pd} / \mathrm{Fe} / \mathrm{Ir}(111)$ a uma solução não-colinear como mínimo global, mas diferentes estados cujos momentos de spin desviam-se do arranjo colinear foram encontrados como soluções metaestáveis. Por sua vez, as fortes interações de DM contribuem para a emergência de uma configuração em espiral de spin como estado fundamental do sistema, sobretudo porque os acoplamentos de primeiros vizinhos Fe-Fe mostraram-se quase paralelos à superfície de $\operatorname{Ir}(111)$, atuando em favor da rotação local dos momentos de spin na direção in-plane.

Ainda sobre a bicamada de $\mathrm{Pd} / \mathrm{Fe} / \mathrm{Ir}(111)$, obtivemos um diagrama de fases baseado na carga topológica total $Q$ do sistema, bem como nos pontos de inflexão associados às susceptibilidades magnéticas $\chi(T)$, que indicam as temperaturas críticas $T_{c}$ nas quais se verificam transições de fase. A partir desses resultados, sugerimos que no intervalo de $1 \mathrm{~T} \lesssim B \lesssim 3 \mathrm{~T}$ para campos magnéticos externos $B$ aplicados (e sendo $T<T_{c}$ ), a camada de Fe apresenta uma configuração caracterizada por redes de skyrmions, nas quais tanto o tamanho quanto o formato dessas estruturas magnéticas e sua distância variam com a intensidade do campo. Mais especificamente, verificamos que os skyrmions passam de um formato elíptico para circular com o aumento de $B$, diminuindo o tamanho de seu eixo maior. Por outro lado, também à medida em que $B$ aumenta, a rede de skyrmions torna-se cada vez mais esparsa - indicando, portanto, uma transição suave para o estado FM (com $B>3 \mathrm{~T}$ ), devido à saturação da magnetização. Para temperaturas aproximadamente superiores aos valores de $T_{c}$ calculados para cada valor de $B$, o sistema torna-se paramagnético.

Por sua vez, a bicamada de $\mathrm{Pd} / \mathrm{Co} / \mathrm{Ir}(111)$, embora já tenha sido observada anteriormente como um sistema que apresenta um estado fundamental FM, demonstrou possuir requisitos que a literatura classifica como importantes para a emergência de um magnetismo não-colinear. Dentre elas, podemos citar um forte acoplamento de DM entre primeiros vizinhos Co-Co e inclusive para vizinhanças mais distantes, exibindo um comportamento de longo alcance mediado pelos elétrons de condução do Co através das bandas $5 d$ do 
Ir. Entretanto, como esperado, nossos cálculos de primeiros princípios e as simulações de dinâmica de spin para esse sistema sempre resultaram em uma configuração FM. A partir de um entendimento mais amplo de sua estrutura eletrônica, verificamos que, ao contrário da bicamada de $\mathrm{Pd} / \mathrm{Fe} / \mathrm{Ir}(111)$, não se observa uma frustração magnética significativa baseada na competição FM-AFM entre as interações de troca $\mathcal{J}_{\text {Co-Co }}$. Além disso, as interações de DM demonstram possuir condições desfavoráveis para o surgimento de uma não-colinearidade nos momentos de spin, haja vista que, por exemplo, o vetor $\mathbf{D}_{\mathrm{Co}-\mathrm{Co}}$ de primeiros vizinhos direciona-se quase perpendicularmente ao plano de superfície (outof-plane). Uma análise angular mostrou que, sendo a configuração de menor energia dos spins FM, a magnitude do produto vetorial $\left(\hat{\mathbf{e}}_{i} \times \hat{\mathbf{e}}_{j}\right)$ tende a zero, praticamente anulando o termo de DM na Hamiltoniana de spin.

Utilizando a mesma metodologia (RS-LMTO-ASA + UppASD), também foram analisadas outras multicamadas magnéticas limpas, com o intuito de encontrar propriedades interessantes e/ou comuns para o surgimento (ou não) de skyrmions nestes sistemas. A partir dos resultados obtidos, encontramos duas conclusões relevantes: $(i)$ os substratos com estrutura $f c c(111)$ demonstraram uma maior chance de apresentar skyrmions em seu diagrama de fase $\left(\mathrm{Pd} / \mathrm{Fe} / \operatorname{Ir}(111), \mathrm{Co} / \mathrm{Ni} / \mathrm{Cu}(111), \mathrm{Ni} / \operatorname{Ir}_{n} / \mathrm{Pt}(111)\right.$, e $\left.\mathrm{Fe} / \mathrm{Rh} / \mathrm{Ir}(111)\right)$, e sugere-se que há uma relação deste fato com a falta de regras específicas de Moriya para os vetores DM neste tipo de superfície; e (ii) nos sistemas AFM (que não apresentaram skyrmions), a evolução da configuração de espiral de spin para um estado de Néel é devida, entre outros fatores, ao aumento da magnitude AFM da interação $\mathcal{J}_{i j}$ e da relação $\frac{\mathcal{J}}{|\mathbf{D}|}$ entre primeiros vizinhos Fe-Fe ou Mn-Mn.

Após a implementação do damping no espaço-real, tratou-se de calculá-lo para sistemas inéditos. Em um primeiro momento, e com o objetivo de verificar a influência de defeitos $4 d$ e $5 d$ em $\alpha_{\text {tot }}$ e $\alpha_{\text {onsite }}$, comparou-se o damping calculado para a superfície pura de $\mathrm{Pd} / \mathrm{Fe} / \mathrm{Ir}(111)$ (cuja estrutura eletrônica já havia sido explorada) com aquele calculado para o mesmo sistema contendo defeitos de Pd, Ir e algumas terras-raras. Essa comparação permitiu constatar que elementos com elevada interação spin-órbita (por exemplo, o Ir) aumentam localmente o damping na camada de Fe (sobretudo em $\alpha_{t o t}$ ), indicando que há uma dissipação maior de energia na dinâmica de spins naquele local (i.e., um menor tempo de precessão). Isso pode estar relacionado, então, ao fato de estes defeitos serem centros de pinning de estruturas magnéticas, tais como skyrmions.

Em um segundo momento, investigou-se a recente e importante observação de uma anisotropia gigante de damping para a superfície de $\mathrm{Fe}_{50} \mathrm{Co}_{50}(100)$ [255]. Os resultados teóricos obtidos neste trabalho demonstraram, em geral, uma elevada anisotropia de $\alpha_{\text {tot }}$ 
quando a magnetização varia entre as direções [010] e [110] - de até $200 \%$ na razão entre os valores máximo e mínimo de damping. Os resultados demonstram que essa elevada anisotropia vem particularmente da camada mais externa (próxima ao vácuo). Outras origens foram também exploradas, primordialmente relacionadas à presença de interfaces, composição da liga e distorções na estrutura local. Encontrou-se que: (i) a presença de Co introduz um elevado spin-órbita e pode localmente modificar os termos de damping nãolocais $\left(\alpha_{i j}\right)$; (ii) a aleatoriedade de Co na liga pode aumentar modestamente a anisotropia como um efeito direto da possível existência de regiões com concentração maior de Co; (iii) na superfície, a distribuição das espécies de Fe/Co aumenta o damping quando mais átomos de Fe estão presentes na camada mais externa; e (iv) a existência de distorções tetragonais ("bct-like") locais atua em favor do aumento dos valores absolutos de $\alpha_{t o t}$, sobretudo modificando o $\alpha_{\text {onsite }}$ do átomo de referência.

O momento atual é promissor para o estudo do magnetismo em escala nanométrica, uma vez que experimentos surgem, constantemente, com novos efeitos e fenômenos observados e de potencial aplicação no desenvolvimento de dispositivos baseados em spintrônica. Em particular, uma nova área desponta como um importante caminho para a gravação magnética: a skyrmiônica. E, no contexto destas estruturas de spins, o damping surge como um relevante parâmetro de controle dinâmico. Os resultados apresentados nesta tese, bem como as implementações realizadas, abrem caminho para um conhecimento mais aprofundado das origens e dinâmica de skyrmions em sistemas metálicos, e dos mecanismos responsáveis pelo damping e sua anisotropia, permitindo avanços em direção ao desenho racional de novos dispositivos de gravação de informação, fundamentais em nosso cotidiano. 


\section{Bibliografia}

[1] J. Hirschenberger, Geschichte der Philosophie (Verlag Herder, 1980).

[2] G. Reiss and A. Hütten, Nat. Mater. 4, 725 (2005).

[3] J. Liu, T. Xia, S. Wang, G. Yang, B. Dong, C. Wang, Q. Ma, Y. Sun, and R. Wang, Nanoscale 8, 11432 (2016).

[4] C. Antoniak, M. E. Gruner, M. Spasova, A. V. Trunova, F. M. Römer, A. Warland, B. Krumme, K. Fauth, S. Sun, P. Entel, M. Farle, and H. Wende, Nat. Commun. 2, 528 (2011).

[5] C. Bergemann, D. Müller-Schulte, J. Oster, L. à Brassard, and A. S. Lübbe, J. Magn. Magn. Mater. 194, 45 (1999).

[6] L. Gao, J. Zhuang, L. Nie, J. Zhang, Y. Zhang, N. Gu, T. Wang, J. Feng, D. Yang, S. Perrett, and X. Yan, Nat. Nanotechnol. 2, 577 (2007).

[7] A. Varela, G. Oliveira, F. Souza Jr., C. Rodrigues, and M. Costa, Polym. Eng. Sci. 53, 44 (2013).

[8] A. A. Khajetoorians, J. Wiebe, B. Chilian, and R. Wiesendanger, Science 332, 1062 (2011).

[9] I. M. Miron, K. Garello, G. Gaudin, P.-J. Zermatten, M. V. Costache, S. Auffret, S. Bandiera, B. Rodmacq, A. Schuhl, and P. Gambardella, Nature 476, 189 (2011).

[10] A. Fert, Rev. Mod. Phys. 80, 1517 (2008).

[11] P. A. Grünberg, Rev. Mod. Phys. 80, 1531 (2008).

[12] A. Moser, K. Takano, D. T. Margulies, M. Albrecht, Y. Sonobe, Y. Ikeda, S. Sun, and E. E. Fullerton, J. Phys. D: Appl. Phys. 35, R157 (2002).

[13] R. Wiesendanger, Rev. Mod. Phys. 81, 1495 (2009). 
[14] G. Binnig, C. F. Quate, and C. Gerber, Phys. Rev. Lett. 56, 930 (1986).

[15] G. Binnig, H. Rohrer, C. Gerber, and E. Weibel, Phys. Rev. Lett. 49, 57 (1982).

[16] C. Chen, F. Sette, Y. Ma, and S. Modesti, Phys. Rev. B 42, 7262 (1990).

[17] W. L. O’Brien and B. P. Tonner, Phys. Rev. B 50, 12672 (1994).

[18] C. Walter, Sci. Am. 293, 32.

[19] T. Moriya, Spin Fluctuations in Itinerant Electron Magnetism (Springer-Verlag Berlin Heidelberg, Germany, 1985).

[20] F. Bloch, Z. Phys. 57, 545 (1929).

[21] S. A. Wolf, Science 294, 1488 (2001).

[22] R. Duine, Nat. Nanotechnol. 8, 800 (2013).

[23] N. S. Kiselev, A. N. Bogdanov, R. Schäfer, and U. K. Rößler, J. Phys. D.: Appl. Phys. 44, 392001 (2011).

[24] F. Jonietz, S. Mühlbauer, C. Pfleiderer, A. Neubauer, W. Münzer, A. Bauer, T. Adams, R. Georgii, P. Böni, R. A. Duine, K. Everschor, M. Garst, and A. Rosch, Science 330, 1648 (2010).

[25] A. Rosch, Nat. Nanotechnol. 8, 160 (2013).

[26] T. Schulz, R. Ritz, A. Bauer, M. Halder, M. Wagner, C. Franz, C. Pfleiderer, K. Everschor, M. Garst, and A. Rosch, Nat. Phys. 8, 301 (2012).

[27] X. Zhang, G. P. Zhao, H. Fangohr, J. P. Liu, W. X. Xia, J. Xia, and F. J. Morvan, Sci. Rep. 5, 7643 (2015).

[28] S. S. P. Parkin, M. Hayashi, and L. Thomas, Science 320, 190 (2008).

[29] F. Ummelen, H. Swagten, and B. Koopmans, Sci. Rep. 7, 833 (2017).

[30] S. Emori, U. Bauer, S.-M. Ahn, E. Martinez, and G. S. D. Beach, Nat. Mater. 12, 611 (2013).

[31] M. Hayashi, L. Thomas, R. Moriya, C. Rettner, and S. S. P. Parkin, Science 320, 209 (2008).

[32] R. N. Igarashi, I. P. Miranda, L. T. F. Eleno, A. B. Klautau, and H. M. Petrilli, J. Phys.: Condens. Matter 28, 326001 (2016). 
[33] S. Lounis, J. Phys.: Condens. Matter 26, 273201 (2014).

[34] M. M. Bezerra-Neto, M. S. Ribeiro, B. Sanyal, A. Bergman, R. B. Muniz, O. Eriksson, and A. B. Klautau, Sci. Rep. 3, 3054 (2013).

[35] S.-H. Phark, J. Fischer, M. Corbetta, D. Sander, K. Nakamura, and J. Kirschner, Nat. Commun. 5, 5183 (2014).

[36] R. Cardias, M. M. Bezerra-Neto, M. S. Ribeiro, A. Bergman, A. Szilva, O. Eriksson, and A. B. Klautau, Phys. Rev. B 93, 014438 (2016).

[37] S. Lounis, P. H. Dederichs, and S. Blügel, Phys. Rev. Lett. 101, 107204 (2008).

[38] S. Holzberger, T. Schuh, S. Blügel, S. Lounis, and W. Wulfhekel, Phys. Rev. Lett. 110, 157206 (2013).

[39] R. N. Igarashi, A. B. Klautau, R. B. Muniz, B. Sanyal, and H. M. Petrilli, Phys. Rev. B 85, 014436 (2012).

[40] M. S. Ribeiro, G. B. Corrêa Jr., A. Bergman, L. Nordström, O. Eriksson, and A. B. Klautau, Phys. Rev. B 83, 014406 (2011).

[41] S. Krause and R. Wiesendanger, Nat. Mater. 15, 493 (2016).

[42] S. Mühlbauer, B. Binz, F. Jonietz, C. Pfleiderer, A. Rosch, A. Neubauer, R. Georgii, and P. Böni, Science 323, 915 (2009).

[43] R. Tomasello, E. Martinez, R. Zivieri, L. Torres, M. Carpentieri, and G. Finocchio, Sci. Rep. 4, 6784 (2015).

[44] J. Sampaio, V. Cros, S. Rohart, A. Thiaville, and A. Fert, Nat. Nanotechnol. 8, 839 (2013).

[45] W. Kang, Y. Huang, C. Zheng, W. Lv, N. Lei, Y. Zhang, X. Zhang, Y. Zhou, and W. Zhao, Sci. Rep. 6, 23164 (2016).

[46] S. Heinze, K. Von Bergmann, M. Menzel, J. Brede, A. Kubetzka, R. Wiesendanger, G. Bihlmayer, and S. Blügel, Nat. Phys. 7, 713 (2011).

[47] W. Heisenberg, Z. Phys. 49, 619 (1928).

[48] I. E. Dzyaloshinskii, J. Phys. Chem. Solids 4, 241 (1958).

[49] T. Moriya, Phys. Rev. 120, 91 (1960). 
[50] M. Bode, M. Heide, K. Von Bergmann, P. Ferriani, S. Heinze, G. Bihlmayer, A. Kubetzka, O. Pietzsch, S. Blügel, and R. Wiesendanger, Nature 447, 190 (2007).

[51] A. H. MacDonald, S. M. Girvin, and D. T. Yoshioka, Phys. Rev. B 37, 9753 (1988).

[52] N. Romming, C. Hanneken, M. Menzel, J. E. Bickel, B. Wolter, K. von Bergmann, A. Kubetzka, and R. Wiesendanger, Science 341, 636 (2013).

[53] L. Rózsa, E. Simon, K. Palotás, L. Udvardi, and L. Szunyogh, Phys. Rev. B 93, 024417 (2016).

[54] E. Simon, K. Palotás, L. Rózsa, L. Udvardi, and L. Szunyogh, Phys. Rev. B 90, 094410 (2014).

[55] N. Romming, A. Kubetzka, C. Hanneken, K. von Bergmann, and R. Wiesendanger, Phys. Rev. Lett. 114, 177203 (2015).

[56] L. Rózsa, A. Deák, E. Simon, R. Yanes, L. Udvardi, L. Szunyogh, and U. Nowak, Phys. Rev. Lett. 117, 157205 (2016).

[57] U. K. Rößler, A. N. Bogdanov, and C. Pfleiderer, Nature 442, 797 (2006).

[58] R. Lizárraga, L. Nordström, L. Bergqvist, A. Bergman, E. Sjöstedt, P. Mohn, and O. Eriksson, Phys. Rev. Lett. 93, 107205 (2004).

[59] C. Hanneken, F. Otte, A. Kubetzka, B. Dupé, N. Romming, K. von Bergmann, R. Wiesendanger, and S. Heinze, Nat. Nanotechnol. 10, 1039 (2015).

[60] B. Dupé, M. Hoffmann, C. Paillard, and S. Heinze, Nat. Commun. 5, 4030 (2014).

[61] L. V. Dzemiantsova, M. Hortamani, C. Hanneken, A. Kubetzka, K. von Bergmann, and R. Wiesendanger, Phys. Rev. B 86, 094427 (2012).

[62] V. P. Antropov, M. I. Katsnelson, B. N. Harmon, M. Van Schilfgaarde, and D. Kusnezov, Phys. Rev. B 54, 1019 (1996).

[63] T. L. Gilbert, Phys. Rev. 100, 1243 (1955).

[64] C. Etz, M. Costa, O. Eriksson, and A. Bergman, Phys. Rev. B 86, 224401 (2012).

[65] H. Ebert, S. Mankovsky, D. Ködderitzsch, and P. J. Kelly, Phys. Rev. Lett. 107, 066603 (2011).

[66] S. Mankovsky, D. Ködderitzsch, G. Woltersdorf, and H. Ebert, Phys. Rev. B 87, 014430 (2013). 
[67] D. Thonig and J. Henk, New J. Phys. 16, 013032 (2014).

[68] I. Turek, J. Kudrnovský, and V. Drchal, Phys. Rev. B 92, 214407 (2015).

[69] P. Rocha-Rodrigues, S. S. M. Santos, G. N. P. Oliveira, T. Leal, I. P. Miranda, A. M. Santos, J. G. Correia, L. V. C. Assali, H. M. Petrilli, J. P. Araújo, and A. M. L. Lopes, Phys. Rev. B 102, 104115 (2020).

[70] M. L. Marcondes, S. S. M. Santos, I. P. Miranda, P. Rocha-Rodrigues, L. V. C. Assali, A. M. L. Lopes, J. P. Araujo, and H. M. Petrilli, J. Mater. Chem. C 8, $14570(2020)$.

[71] P. Rocha-Rodrigues, S. S. M. Santos, I. P. Miranda, G. N. P. Oliveira, J. G. Correia, L. V. C. Assali, H. M. Petrilli, J. P. Araújo, and A. M. L. Lopes, Phys. Rev. B 101, 064103 (2020).

[72] A. I. Akhiezer et al., Spin Waves (John Wiley \& Sons, Inc., New York, 1968).

[73] P. Weiss, J. Phys. Théorique Appliquée 6, 661 (1907).

[74] A. P. Guimarães, Magnetism and Magnetic Resonance in Solids (John Wiley \& Sons, Inc., New York, 1998).

[75] P. Mohn, Magnetism in the Solid State: An Introduction (Springer, 2003).

[76] R. N. Igarashi, Estudo teórico de nanoestruturas magnéticas em superfícies metálicas, Ph.D. thesis, Universidade de São Paulo (2012).

[77] A. I. Liechtenstein, M. I. Katsnelson, V. P. Antropov, and V. A. Gubanov, J. Magn. Magn. Mater. 67, 65 (1987).

[78] E. C. Stoner, Proc. Math. Phys. Eng. Sci. 169, 339 (1939).

[79] A. B. Klautau, Estudo de Propriedades Locais de Impurezas Substitucionais de Fe em Pd, Sc, Y, Ti e Zr, Master's thesis, Universidade de São Paulo (1995).

[80] P. W. Andersen, Solid State Physics, Vol. 14, edited by F. Seitz and D. Turnbull (Academic Press, New York, 1963) p. 99.

[81] C. Zener, Phys. Rev. 81, 440 (1951).

[82] C. Zener, Phys. Rev. 82, 403 (1951).

[83] H. Häffner, Nature 465, 555 (2010). 
[84] H. T. Diep, ed., Frustrated Spin Systems (World Scientific Publishing Co. Pte. Ltd., 2004).

[85] P. A. M. Dirac, Proc. R. Soc. A 117, 610 (1928).

[86] L. D. Landau et al., Statistical Physics (Pergamon Press Ltd., 1980).

[87] P. W. Anderson, Phys. Rev. 115, 2 (1959).

[88] A. B. Butenko, A. A. Leonov, A. N. Bogdanov, and U. K. Rößler, Phys. Rev. B 80, 134410 (2009).

[89] M. Heide, G. Bihlmayer, and S. Blügel, Physica B Condens. Matter 404, 2678 (2009).

[90] M. Menzel, Y. Mokrousov, R. Wieser, J. E. Bickel, E. Vedmedenko, S. Blügel, S. Heinze, K. von Bergmann, A. Kubetzka, and R. Wiesendanger, Phys. Rev. Lett. 108, 197204 (2012).

[91] S. Polesya, S. Mankovsky, S. Bornemann, D. Ködderitzsch, J. Minár, and H. Ebert, Phys. Rev. B 89, 184414 (2014).

[92] M. Heide, G. Bihlmayer, and S. Blügel, Phys. Rev. B 78, 140403 (2008).

[93] A. Fert, Mater. Sci. Forum 59-60, 439 (1990).

[94] T. H. R. Skyrme, Proc. R. Soc. A 260, 127 (1961).

[95] A. Fert, V. Cros, and J. Sampaio, Nat. Nanotechnol. 8, 152 (2013).

[96] R. Wiesendanger, Nat. Rev. Mater. 1, 16044 (2016).

[97] M. Ezawa, Phys. Rev. Lett. 105, 197202 (2010).

[98] A. Fert, N. Reyren, and V. Cros, Nat. Rev. Mater. 2, 17031 (2017).

[99] S. Seki and M. Mochizuki, Skyrmions in Magnetic Materials, SpringerBriefs in Physics (Springer International Publishing, 2016).

[100] C. Moreau-Luchaire, C. Moutafis, N. Reyren, J. Sampaio, N. Van Horne, C. a. F. Vaz, K. Bouzehouane, K. Garcia, C. Deranlot, P. Warnicke, P. Wohlhüter, J. M. George, J. Raabe, V. Cros, and A. Fert, Nat. Nanotechnol. 11, 444 (2016).

[101] A. A. Abrikosov, Rev. Mod. Phys. 76, 975 (2004). 
[102] J. Brede, N. Atodiresei, V. Caciuc, M. Bazarnik, A. Al-Zubi, S. Blügel, and R. Wiesendanger, Nat. Nanotechnol. 9, 1018 (2014).

[103] A. Brataas, A. D. Kent, and H. Ohno, Nat. Mater. 11, 372 (2012).

[104] J. Slonczewski, J. Magn. Magn. Mater. 159, L1 (1996).

[105] X. Zhang, M. Ezawa, and Y. Zhou, Sci. Rep. 5, 9400 (2015).

[106] J. Zang, M. Mostovoy, J. H. Han, and N. Nagaosa, Phys. Rev. Lett. 107, 136804 (2011).

[107] C. Pfleiderer and A. Rosch, Nature 465, 880 (2010).

[108] X. Z. Yu, Y. Onose, N. Kanazawa, J. H. Park, J. H. Han, Y. Matsui, N. Nagaosa, and Y. Tokura, Nature 465, 901 (2010).

[109] M. Hervé, B. Dupé, R. Lopes, M. Böttcher, M. D. Martins, T. Balashov, L. Gerhard, J. Sinova, and W. Wulfhekel, Nat. Commun. 9, 1015 (2018).

[110] W. Jiang, P. Upadhyaya, W. Zhang, G. Yu, M. B. Jungfleisch, F. Y. Fradin, J. E. Pearson, Y. Tserkovnyak, K. L. Wang, O. Heinonen, S. G. E. te Velthuis, and A. Hoffmann, Science 349, 283 (2015).

[111] J. E. Hirsch, Phys. Rev. Lett. 83, 1834 (1999).

[112] A. Chacon, L. Heinen, M. Halder, A. Bauer, W. Simeth, S. Mühlbauer, H. Berger, M. Garst, A. Rosch, and C. Pfleiderer, Nat. Phys. 14, 936 (2018).

[113] B. Berg and M. Lüscher, Nucl. Phys. B 190, 412 (1981).

[114] S. Blundell, Magnetism in Condensed Matter (Oxford University Press Inc., New York, 2001).

[115] A. P. Guimarães, Principles of Nanomagnetism (Springer-Verlag Heidelberg, Berlin, Germany, 2009).

[116] O. Šipr, S. Bornemann, J. Minár, and H. Ebert, Phys. Rev. B 82, 174414 (2010).

[117] O. Šipr, S. Bornemann, H. Ebert, S. Mankovsky, J. Vackář, and J. Minár, Phys. Rev. B 88, 064411 (2013).

[118] X. Wang, R. Wu, D.-s. Wang, and A. J. Freeman, Phys. Rev. B 54, 61 (1996).

[119] P. Bruno, Phys. Rev. B 39, 865(R) (1989). 
[120] D. C. M. Rodrigues, A. B. Klautau, A. Edström, J. Rusz, L. Nordström, M. Pereiro, B. Hjörvarsson, and O. Eriksson, Phys. Rev. B 97, 224402 (2018).

[121] C. Andersson, B. Sanyal, O. Eriksson, L. Nordström, O. Karis, D. Arvanitis, T. Konishi, E. Holub-Krappe, and J. H. Dunn, Phys. Rev. Lett. 99, 177207 (2007).

[122] M. Born and R. Oppenheimer, Ann. Phys. 389, 457 (1927).

[123] P. Hohenberg and W. Kohn, Phys. Rev. 136, B864 (1964).

[124] W. Kohn et al., Phys. Rev. 140, A1133 (1965).

[125] H. L. Skriver, The LMTO Method: Muffin-Tin Orbitals and Electronic Structure (Springer-Verlag, 1984).

[126] J. D. M. Vianna et al., Teoria Quântica de Moléculas e Sólidos (Livraria da Física, São Paulo, 2004).

[127] U. von Barth and L. Hedin, J. Phys. C: Solid State Phys. 5, 1629 (1972).

[128] O. Gunnarsson and B. I. Lundqvist, Phys. Rev. B 13, 4274 (1976).

[129] K. Capelle, Brazilian J. Phys. 36, 1318 (2006).

[130] O. K. Andersen and O. Jepsen, Phys. Rev. Lett. 53, 2571 (1984).

[131] O. K. Andersen et al., Highlights of Condensed-Matter Theory, edited by F. Bassani, F. Fumi, and M. P. Tosi (North Holland, North Holland, Amsterdam, 1985).

[132] H. M. Petrilli and S. Frota-Pessôa, Phys. Rev. B 48, 7148 (1993).

[133] P. Peduto, S. Frota-Pessôa, and M. Methfessel, Phys. Rev. B 44, 13283 (1991).

[134] S. Frota-Pessôa, Phys. Rev. B 46, 14570 (1992).

[135] R. Haydock, Solid State Physics, edited by H. Ehrenreich, F. Seitz, and D. Turnbull (Academic Press, New York, 1980).

[136] N. Beer and D. G. Pettifor, The Electronic Structure of Complex Systems, edited by W. Temmermann and P. Phariseau (Plenum Press, New York, 1984).

[137] S. Ferreira, Parametrização LMTO-ASA no cálculo de estrutura eletrônica de metais de transição, Master's thesis, Universidade de São Paulo (1989).

[138] A. B. Klautau, Cálculos Ab Initio da Estrutura Eletrônica e Propriedades Magnéticas de Sistemas Metálicos Bidimensionais, Ph.D. thesis, Universidade de São Paulo (2000). 
[139] R. N. Nogueira and H. M. Petrilli, Phys. Rev. B 60, 4120 (1999).

[140] S. Frota-Pessôa and A. B. Klautau, Int. J. Mod. Phys. B 20, 5281 (2006).

[141] I. P. Miranda, Propriedades magnéticas de trímeros de FexCo1-x depositados em Pt(111), Master's thesis, Universidade de São Paulo (2016).

[142] S. Frota-Pessôa, L. A. De Mello, H. M. Petrilli, and A. B. Klautau, Phys. Rev. Lett. 71, 4206 (1993).

[143] R. N. Igarashi, M. M. Bezerra-Neto, L. T. F. Eleno, A. Bergman, A. B. Klautau, O. Eriksson, and H. M. Petrilli, J. Phys.: Condens. Matter 26, 206003 (2014).

[144] A. B. Klautau, P. R. Peduto, and S. Frota-Pessôa, J. Magn. Magn. Mater. 186, 223 (1998).

[145] A. B. Klautau, S. B. Legoas, R. B. Muniz, and S. Frota-Pessôa, Phys. Rev. B 60, 3421 (1999).

[146] M. Aldén, S. Mirbt, H. L. Skriver, N. M. Rosengaard, and B. Johansson, Phys. Rev. B 46, 6303 (1992).

[147] H. L. Skriver and N. M. Rosengaard, Phys. Rev. B 43, 9538 (1991).

[148] P. P. Ewald, Ann. Phys. 369, 253 (1921).

[149] T. Oguchi, K. Terakura, and N. Hamada, J. Phys. F: Met. Phys. 13, 145 (1983).

[150] M. Methfessel and J. Kubler, J. Phys. F Met. Phys. 12, 141 (1982).

[151] J. Gräfenstein and P. Ziesche, Phys. Rev. B 53, 7143 (1996).

[152] P. Lloyd and P. V. Smith, Adv. Phys. 21, 69 (1972).

[153] B. L. Györffy et al., Electrons in Disordered Metals and at Metallic Surfaces, edited by P. Phariseau, B. L. Györffy, and L. Scheire (Plenum Press, New York, 1979).

[154] S. Frota-Pessôa, R. B. Muniz, and J. Kudrnovský, Phys. Rev. B 62, 5293 (2000).

[155] A. T. Costa, R. B. Muniz, and D. L. Mills, Phys. Rev. Lett. 94, 137203 (2005).

[156] A. Szilva, M. Costa, A. Bergman, L. Szunyogh, L. Nordström, and O. Eriksson, Phys. Rev. Lett. 111, 127204 (2013).

[157] R. Cardias, A. Szilva, M. M. Bezerra-Neto, M. S. Ribeiro, A. Bergman, Y. O. Kvashnin, J. Fransson, A. B. Klautau, O. Eriksson, and L. Nordström, Sci. Rep. 10, $1(2020)$. 
[158] A. Szilva, D. Thonig, P. F. Bessarab, Y. O. Kvashnin, D. C. M. Rodrigues, R. Cardias, M. Pereiro, L. Nordström, A. Bergman, A. B. Klautau, and O. Eriksson, Phys. Rev. B 96, 144413 (2017).

[159] W. Kohn and N. Rostoker, Phys. Rev. 94, 1111 (1954).

[160] J. Korringa, Physica 13, 392 (1947).

[161] M. van Schilfgaarde and V. P. Antropov, J. Appl. Phys. 85, 4827 (1999).

[162] V. P. Antropov, B. N. Harmon, and A. N. Smirnov, J. Magn. Magn. Mater. 200, 148 (1999).

[163] R. Cardias, A. Bergman, A. Szilva, Y. O. Kvashnin, J. Fransson, A. B. Klautau, O. Eriksson, and L. Nordström, arXiv:2003.04680 (2020).

[164] B. Skubic, J. Hellsvik, L. Nordström, and O. Eriksson, J. Phys.: Condens. Matter 20, 315203 (2008).

[165] O. Eriksson et al., Atomistic Spin Dynamics: Foundations and Applications (Oxford University Press, 2017).

[166] C. Etz, L. Bergqvist, A. Bergman, A. Taroni, and O. Eriksson, J. Phys.: Condens. Matter 27, 243202 (2015).

[167] M. A. W. Schoen, D. Thonig, M. L. Schneider, T. J. Silva, H. T. Nembach, O. Eriksson, O. Karis, and J. M. Shaw, Nat. Phys. 12, 839 (2016).

[168] A. Bergman, B. Skubic, J. Hellsvik, L. Nordström, A. Delin, and O. Eriksson, Phys. Rev. B 83, 224429 (2011).

[169] S. Woo, K. Litzius, B. Krüger, M.-Y. Im, L. Caretta, K. Richter, M. Mann, A. Krone, R. M. Reeve, M. Weigand, P. Agrawal, I. Lemesh, M.-A. Mawass, P. Fischer, M. Kläui, and G. S. D. Beach, Nat. Mater. 15, 501 (2016).

[170] J. H. Mentink, M. V. Tretyakov, A. Fasolino, M. I. Katsnelson, and T. Rasing, J. Phys.: Condens. Matter 22, 176001 (2010).

[171] J. L. García-Palacios and F. J. Lázaro, Phys. Rev. B 58, 14937 (1998).

[172] H. Nyquist, Phys. Rev. 32, 110 (1928).

[173] N. Metropolis, A. W. Rosenbluth, M. N. Rosenbluth, A. H. Teller, and E. Teller, J. Chem. Phys. 21, 1087 (1953). 
[174] Y. Li, N. Kanazawa, X. Z. Yu, A. Tsukazaki, M. Kawasaki, M. Ichikawa, X. F. Jin, F. Kagawa, and Y. Tokura, Phys. Rev. Lett. 110, 117202 (2013).

[175] A. Neubauer, C. Pfleiderer, B. Binz, A. Rosch, R. Ritz, P. Niklowitz, and P. Böni, Phys. Rev. Lett. 102, 186602 (2009).

[176] W. Weber, D. Wesner, G. Güntherodt, and U. Linke, Phys. Rev. Lett. 66, 942 (1991).

[177] R. Tyer, G. Van der Laan, W. M. Temmerman, Z. Szotek, and H. Ebert, Phys. Rev. B 67, 104409 (2003).

[178] G. Kresse and D. Joubert, Phys. Rev. B 59, 1758 (1999).

[179] P. E. Blöchl, Phys. Rev. B 50, 17953 (1994).

[180] P. Giannozzi et al., J. Phys.: Condens. Matter 21, 395502 (2009).

[181] P. Giannozzi et al., J. Phys.: Condens. Matter 29, 465901 (2017).

[182] D. Smith, J. Magn. Magn. Mater. 1, 214 (1976).

[183] A. Fert and M. Levy, Phys. Rev. Lett. 44, 1538 (1980).

[184] A. A. Khajetoorians, J. Wiebe, B. Chilian, and R. Wiesendanger, Nat. Commun. 7, 10620 (2016).

[185] A. Crépieux and C. Lacroix, J. Magn. Magn. Mater. 182, 341 (1998).

[186] S. B. Legoas and S. Frota-Pessôa, Phys. Rev. B 61, 12566 (2000).

[187] A. Bergman, L. Nordström, A. B. Klautau, S. Frota-Pessôa, and O. Eriksson, Phys. Rev. B 75, 224425 (2007).

[188] N. W. Ashcroft and N. D. Mermin, Solid State Physics (Saunders College, Florida, 1976).

[189] P. F. Bessarab, G. P. Müller, I. S. Lobanov, F. N. Rybakov, N. S. Kiselev, H. Jónsson, V. M. Uzdin, S. Blügel, L. Bergqvist, and A. Delin, Sci. Rep. 8, 3433 (2018).

[190] S. Bhattacharjee, A. Bergman, A. Taroni, J. Hellsvik, B. Sanyal, and O. Eriksson, Phys. Rev. X 2, 011013 (2012).

[191] B. Dupé, G. Bihlmayer, M. Böttcher, S. Blügel, and S. Heinze, Nat. Commun. 7, 11779 (2016). 
[192] T. Okubo, S. Chung, and H. Kawamura, Phys. Rev. Lett. 108, 017206 (2012).

[193] I. P. Miranda, R. N. Igarashi, A. B. Klautau, and H. M. Petrilli, J. Magn. Magn. Mater. 441, 222 (2017).

[194] Y. O. Kvashnin, R. Cardias, A. Szilva, I. Di Marco, M. I. Katsnelson, A. I. Lichtenstein, L. Nordström, A. Klautau, and O. Eriksson, Phys. Rev. Lett. 116, 217202 (2016).

[195] P. A. Igoshev, A. V. Efremov, and A. A. Katanin, Phys. Rev. B 91, 195123 (2015).

[196] C. Holl, M. Knol, M. Pratzer, J. Chico, I. L. Fernandes, S. Lounis, and M. Morgenstern, Nat. Commun. 11, 1 (2020).

[197] C. Psaroudaki, S. Hoffman, J. Klinovaja, and D. Loss, Phys. Rev. X 7, 041045 (2017).

[198] J. Müller and A. Rosch, Phys. Rev. B 91, 054410 (2015).

[199] S.-Z. Lin, C. Reichhardt, C. D. Batista, and A. Saxena, Phys. Rev. B 87, 214419 (2013).

[200] I. L. Fernandes, J. Chico, and S. Lounis, J. Phys.: Condens. Matter 32, 425802 (2020).

[201] I. G. Arjana, I. L. Fernandes, J. Chico, and S. Lounis, Sci. Rep. 10, 1 (2020).

[202] N. Romming, H. Pralow, A. Kubetzka, M. Hoffmann, S. von Malottki, S. Meyer, B. Dupé, R. Wiesendanger, K. von Bergmann, and S. Heinze, Phys. Rev. Lett. 120, 207201 (2018).

[203] P. Kurz, G. Bihlmayer, K. Hirai, and S. Blügel, Phys. Rev. Lett. 86, 1106 (2001).

[204] A. Belabbes, G. Bihlmayer, F. Bechstedt, S. Blügel, and A. Manchon, Phys. Rev. Lett. 117, 247202 (2016).

[205] E. Simon, K. Palotás, B. Ujfalussy, A. Deák, G. Stocks, and L. Szunyogh, J. Phys.: Condens. Matter 26, 186001 (2014).

[206] J. Li, A. Tan, K. Moon, A. Doran, M. Marcus, A. Young, E. Arenholz, S. Ma, R. Yang, C. Hwang, and Z. Q. Qiu, Nat. Commun. 5, 4704 (2014).

[207] B. Hardrat, A. Al-Zubi, P. Ferriani, S. Blügel, G. Bihlmayer, and S. Heinze, Phys. Rev. B 79, 094411 (2009). 
[208] A. Al-Zubi, G. Bihlmayer, and S. Blügel, Phys. Status Solidi B 248, 2242 (2011).

[209] K. von Bergmann, A. Kubetzka, O. Pietzsch, and R. Wiesendanger, J. Phys.: Condens. Matter 26, 394002 (2014).

[210] J. Spethmann, S. Meyer, K. von Bergmann, R. Wiesendanger, S. Heinze, and A. Kubetzka, Phys. Rev. Lett. 124, 227203 (2020).

[211] W. Legrand, D. Maccariello, F. Ajejas, S. Collin, A. Vecchiola, K. Bouzehouane, N. Reyren, V. Cros, and A. Fert, Nat. Mater. 19, 34 (2020).

[212] X. Zhang, Y. Zhou, and M. Ezawa, Sci. Rep. 6, 24795 (2016).

[213] W. Jiang, X. Zhang, G. Yu, W. Zhang, X. Wang, M. B. Jungfleisch, J. E. Pearson, X. Cheng, O. Heinonen, K. L. Wang, Y. Zhou, A. Hoffmann, and S. G. E. te Velthuis, Nat. Phys. 13, 162 (2017).

[214] X. Zhang, Y. Zhou, and M. Ezawa, Nat. Commun. 7, 1 (2016).

[215] V. Kamberský, Czech. J. Phys. 34, 1111 (1984).

[216] V. Kamberský, Phys. Rev. B 76, 134416 (2007).

[217] K. Gilmore, Y. Idzerda, and M. D. Stiles, Phys. Rev. Lett. 99, 027204 (2007).

[218] V. Kamberský, Czech. J. Phys. B 26, 1366 (1976).

[219] J. Kuneš and V. Kamberský, Phys. Rev. B 65, 212411 (2002).

[220] I. P. Miranda, A. B. Klautau, A. Bergman, D. Thonig, H. M. Petrilli, and O. Eriksson, arXiv:2101.02794 (2021).

[221] A. T. Costa and R. B. Muniz, Phys. Rev. B 92, 014419 (2015).

[222] X. Ma, L. Ma, P. He, H. Zhao, S. Zhou, and G. Lüpke, Phys. Rev. B 91, 014438 (2015).

[223] D. M. Edwards, J. Phys.: Condens. Matter 28, 086004 (2016).

[224] B. Khodadadi, A. Rai, A. Sapkota, A. Srivastava, B. Nepal, Y. Lim, D. A. Smith, C. Mewes, S. Budhathoki, A. J. Hauser, M. Gao, J.-F. Li, D. D. Viehland, Z. Jiang, J. J. Heremans, P. V. Balachandran, T. Mewes, and S. Emori, Phys. Rev. Lett. 124, 157201 (2020).

[225] D. Thonig, Y. Kvashnin, O. Eriksson, and M. Pereiro, Phys. Rev. Mater. 2, 013801 (2018). 
[226] S. Mankovsky, S. Wimmer, and H. Ebert, Phys. Rev. B 98, 104406 (2018).

[227] M. Oogane, T. Kubota, H. Naganuma, and Y. Ando, J. Phys. D: Appl. Phys 48, $164012(2015)$.

[228] M. A. W. Schoen, J. Lucassen, H. T. Nembach, B. Koopmans, T. J. Silva, C. H. Back, and J. M. Shaw, Phys. Rev. B 95, 134411 (2017).

[229] C. Kittel, Phys. Rev. 76, 743 (1949).

[230] S. M. Bhagat and P. Lubitz, Phys. Rev. B 10, 179 (1974).

[231] N. Umetsu, D. Miura, and A. Sakuma, J. Phys. Soc. Japan 81, 114716 (2012).

[232] H. M. Petrilli, Método de Cálculo do Gradiente de Campo Elétrico no Núcleo para Sistemas sem Simetria, Ph.D. thesis, Universidade de São Paulo (1989).

[233] B. M'Passi-Mabiala, S. Meza-Aguilar, and C. Demangeat, Surf. Sci. 518, 104 (2002).

[234] B. Min, T. Oguchi, and A. J. Freeman, Phys. Rev. B 33, 7852(R) (1986).

[235] R. Chimata, E. K. Delczeg-Czirjak, A. Szilva, R. Cardias, Y. O. Kvashnin, M. Pereiro, S. Mankovsky, H. Ebert, D. Thonig, B. Sanyal, A. B. Klautau, and O. Eriksson, Phys. Rev. B 95, 214417 (2017).

[236] J. M. Wills and R. Cooper, Phys. Rev. B 36, 3809 (1987).

[237] C. Vittoria, S. D. Yoon, and A. Widom, Phys. Rev. B 81, 014412 (2010).

[238] O. d'Allivy Kelly, A. Anane, R. Bernard, J. Ben Youssef, C. Hahn, A. H. Molpeceres, C. Carrétéro, E. Jacquet, C. Deranlot, P. Bortolotti, R. Lebourgeois, J.-C. Mage1, G. de Loubens, O. Klein, V. Cros, and A. Fert, Appl. Phys. Lett. 103, 082408 (2013).

[239] K. Momma and F. Izumi, J. Appl. Crystallogr. 44, 1272 (2011).

[240] S. Mizukami, D. Watanabe, M. Oogane, Y. Ando, Y. Miura, M. Shirai, and T. Miyazaki, J. Appl. Phys. 105, 07D306 (2009).

[241] S. Mizukami, D. Watanabe, M. Oogane, Y. Ando, Y. Miura, M. Shirai, and T. Miyazaki, Jpn. J. Appl. Phys. 45, 3889 (2006).

[242] R. Weber, D.-S. Han, I. Boventer, S. Jaiswal, R. Lebrun, G. Jakob, and M. Kläui, J. Phys. D: Appl. Phys. 52, 325001 (2019). 
[243] R. Mandal, J. W. Jung, K. Masuda, Y. K. Takahashi, Y. Sakuraba, S. Kasai, Y. Miura, T. Ohkubo, and K. Hono, Appl. Phys. Lett. 113, 232406 (2018).

[244] M. Acet, C. John, and E. F. Wassermann, J. Appl. Phys. 70, 6556 (1991).

[245] J. M. Shaw, H. T. Nembach, and T. J. Silva, Appl. Phys. Lett. 99, 012503 (2011).

[246] E. Barati, M. Cinal, D. M. Edwards, and A. Umerski, Phys. Rev. B 90, 014420 (2014).

[247] L. Chen, S. Mankovsky, S. Wimmer, M. Schoen, H. Körner, M. Kronseder, D. Schuh, D. Bougeard, H. Ebert, D. Weiss, and C. H. Back, Nat. Phys. 14, 490 (2018).

[248] T. Seki, J. Shimada, S. Iihama, M. Tsujikawa, T. Koganezawa, A. Shioda, T. Tashiro, W. Zhou, S. Mizukami, M. Shirai, and K. Takanashi, J. Phys. Soc. Jpn. 86, 074710 (2017).

[249] M. Arora, C. Fowley, T. McKinnon, E. Kowalska, V. Sluka, A. M. Deac, B. Heinrich, and E. Girt, IEEE Magn. Lett. 8, 1 (2016).

[250] F. S. M. Guimarães, J. R. Suckert, J. Chico, J. Bouaziz, M. dos Santos Dias, and S. Lounis, J. Phys.: Condens. Matter 31, 255802 (2019).

[251] A. Bergman, L. Nordström, A. B. Klautau, S. Frota-Pessôa, and O. Eriksson, Phys. Rev. B 73, 174434 (2006).

[252] P. Blaha et al., "WIEN2K: An Augmented Plane Wave and Local Orbitals Program for Calculating Crystal Properties," (2001).

[253] I. Lima Fernandes et al., Nat. Commun. 9, 4395 (2018).

[254] J. Iwasaki, M. Mochizuki, and N. Nagaosa, Nat. Commun. 4, 1 (2013).

[255] Y. Li, F. Zeng, S. S.-L. Zhang, H. Shin, H. Saglam, V. Karakas, O. Ozatay, J. E. Pearson, O. G. Heinonen, Y. Wu, A. Hoffmann, and W. Zhang, Phys. Rev. Lett. 122, 117203 (2019).

[256] K. Shikada, M. Ohtake, F. Kirino, and M. Futamoto, J. Appl. Phys. 105, 07C303 (2009).

[257] M. N. Baibich, J. M. Broto, A. Fert, F. N. Van Dau, F. Petroff, P. Etienne, G. Creuzet, A. Friederich, and J. Chazelas, Phys. Rev. Lett. 61, 2472 (1988).

[258] I. Turek, J. Kudrnovskỳ, and K. Carva, Phys. Rev. B 86, 174430 (2012). 
[259] S. S. A. Razee, J. B. Staunton, B. Ginatempo, F. J. Pinski, and E. Bruno, Phys. Rev. Lett. 82, 5369 (1999).

[260] M. A. Ruderman and C. Kittel, Phys. Rev. 96, 99 (1954).

[261] T. Kasuya, Prog. Theor. Phys. 16, 45 (1956).

[262] K. Yosida, Phys. Rev. 106, 893 (1957).

[263] J. J. Préjean, M. J. Joliclerc, and P. Monod, J. Phys. 41, 427 (1980).

[264] M. Heide et al., $\Psi_{k}$ Highlight 78, 1 (2006).

[265] O. K. Andersen, Phys. Rev. B 12, 3060 (1975).

[266] M. Methfessel, D. Hennig, and M. Scheffler, Phys. Rev. B 46, 4816 (1992).

[267] S. Gasiorowicz, Quantum Physics (John Wiley \& Sons, Inc., 1974).

[268] S. Blügel et al., "Full-Potential Linearized Augmented Planewave Method," (2006).

[269] S. B. Legoas, Estudo das Propriedades Magnéticas e Hiperfinas de Impurezas Substitucionais em Sistemas Metálicos Complexos, Ph.D. thesis, Universidade de São Paulo (1998).

[270] C. Lanczos, J. Res. Natl. Bur. Stand. (1934). 45, 255 (1950).

[271] E. Dagotto, Rev. Mod. Phys. 66, 763 (1994).

[272] H. M. Petrilli, Aplicação do Método de Recorrência para o Cálculo do Gradiente de Campo Elétrico no Núcleo em Metais de Transição, Master's thesis, Universidade de São Paulo (1985).

[273] S. Bornemann, O. Šipr, S. Mankovsky, S. Polesya, J. Staunton, W. Wurth, H. Ebert, and J. Minár, Phys. Rev. B 86, 104436 (2012).

[274] S. Frota-Pessôa, Phys. Rev. B 69, 104401 (2004).

[275] A. Klautau and S. Frota-Pessôa, Surf. Sci. 497, 385 (2002).

[276] D. C. M. Rodrigues, A. Szilva, A. B. Klautau, A. Bergman, O. Eriksson, and C. Etz, Phys. Rev. B 94, 014413 (2016).

[277] C. Hanneken, A. Kubetzka, K. Von Bergmann, and R. Wiesendanger, New J. Phys. 18, 055009 (2016). 


\section{Apêndice A}

\section{Interação de DM como efeito do spin-órbita}

Um importante resultado, que estendeu a aplicabilidade da interação de troca anisotrópica (Eq. 2.12) para sistemas formados puramente por metais de transição, foi obtido por Fert e Levy em 1980 [183]. Em 1976, D. A. Smith [182] demonstrou, no caso especial de duas impurezas distantes com troca indireta de Ruderman-Kittel-Kasuya-Yosida (RKKY) [260-262] via um substrato não-magnético, que a interação entre os átomos $A$ e $B$ (com spins $\mathbf{S}_{A}$ e $\mathbf{S}_{B}$ ) necessariamente envolve um sítio da superfície cujo acoplamento spin-órbita eletrônico é proporcional a $\mathbf{l} \cdot \mathbf{s}$, originando um termo de interação do tipo $\left(\mathbf{S}_{A} \cdot \mathbf{s}\right)(\mathbf{l} \cdot \mathbf{s})\left(\mathbf{s} \cdot \mathbf{S}_{B}\right)$. Nele, $\mathbf{l}$ e s representam, nesta ordem, o momento orbital angular e o spin dos elétrons de condução, responsáveis pelo mecanismo RKKY. Tomando o traço de $\left(\mathbf{S}_{A} \cdot \mathbf{s}\right)(\mathbf{l} \cdot \mathbf{s})\left(\mathbf{s} \cdot \mathbf{S}_{B}\right)$ sobre a variável de spin $\mathbf{s}$, este termo pode ser reescrito como $\left(-\frac{i}{4}\right) \mathbf{l} \cdot\left(\mathbf{S}_{A} \times \mathbf{S}_{B}\right) \equiv \mathbf{D} \cdot\left(\mathbf{S}_{A} \times \mathbf{S}_{B}\right)$, que manifesta a forma da interação de DzyaloshinskiiMoriya (DM). Posteriormente, algumas observações experimentais [263] indicavam que a adição de impurezas não-magnéticas com elevado acoplamento spin-órbita (i.e. Au e $\mathrm{Pt}$ ) em uma liga de $\mathrm{Cu}-\mathrm{Mn}$ causavam um aumento do campo anisotrópico - mantendo, portanto, a magnetização remanescente da aplicação de um campo magnético externo.

De posse destes resultados, Fert e Levy [183] consideraram um modelo de troca local entre um elétron de condução ( spin $\mathbf{s}$, momento orbital angular $\mathbf{l}$ ) e dois átomos com spins $\mathbf{S}_{A}$ e $\mathbf{S}_{B}$, respectivamente posicionados nos sítios $\mathbf{R}_{A}$ e $\mathbf{R}_{B}$ (Fig. A.1). A presença da impureza não-magnética (na posição $\mathbf{R}=0$ ) sujeita este elétron ao espalhamento (scattering) do spin devido à interação spin-órbita, que acopla $\mathbf{s}$ à determinadas coordenadas 
espaciais. Assim, o modelo leva ao potencial perturbativo dado por:

$$
V^{\prime}=-\mathcal{J}_{s-d} \delta\left(\mathbf{r}-\mathbf{R}_{A}\right) \mathbf{s} \cdot \mathbf{S}_{A}-\mathcal{J}_{s-d} \delta\left(\mathbf{r}-\mathbf{R}_{B}\right) \mathbf{s} \cdot \mathbf{S}_{B}+\xi(\mathbf{r}) \mathbf{s} \cdot \mathbf{l}
$$
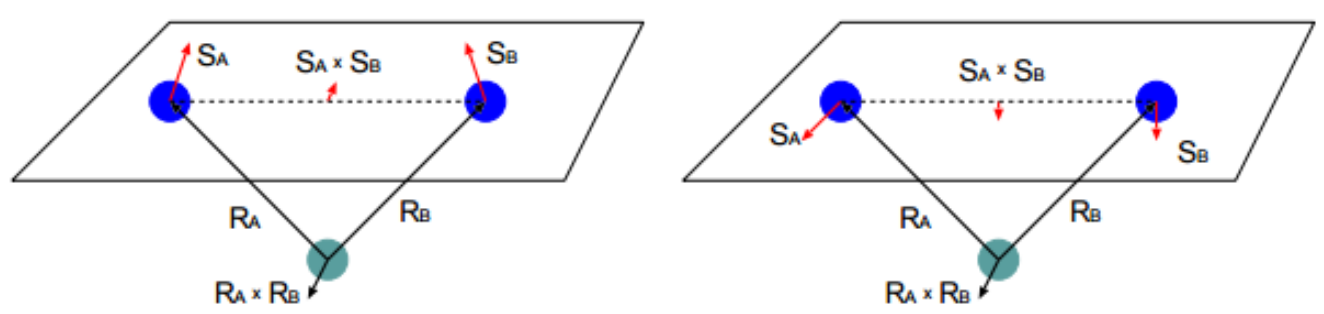

Figura A.1: Representação de dois adátomos $(A$ e $B)$ em duas situações magnéticas em uma superfície não-magnética interagindo com um átomo pertencente à esta superfície, localizados, respectivamente, nas posições $\mathbf{R}_{A}, \mathbf{R}_{B}$, e $\mathbf{R}=0$ [264]. Os momentos de spin de $A$ e $B$ são levemente inclinados devido ao termo proporcional a $\mathbf{S}_{A} \times \mathbf{S}_{B}$ (Eq. A.2).

onde $\xi$, seguindo a notação usual, representa a magnitude (strength) do acoplamento spin-órbita (seção 2.4). Empregando a teoria de perturbação de segunda ordem em $V^{\prime}$, deriva-se o processo no qual um elétron é polarizado pelo spin $\mathbf{S}_{A}$, espalhado no sítio da impureza, e finalmente polariza o spin $\mathbf{S}_{B}$. A partir da identidade $\mathbf{s}=\frac{1}{2} \hbar \boldsymbol{\sigma}$, o traço dos estados eletrônicos de spin, que compõem o termo de correção encontrado na referência [183], podem ser reescritos como $\operatorname{Tr}\left(\mathbf{S}_{A} \cdot \mathbf{s}\right) \mathbf{s}\left(\mathbf{S}_{B} \cdot \mathbf{S}_{B}\right) \propto i\left(\mathbf{S}_{A} \times \mathbf{S}_{B}\right)$. De fato, o termo de energia dominante no modelo de Fert e Levy é [183]:

$$
\mathcal{H}_{D M} \propto \frac{\operatorname{sen}\left[k_{F}\left(R_{A}+R_{B}+R_{A B}\right)+\eta\right] \hat{\mathbf{R}}_{A} \cdot \hat{\mathbf{R}}_{B}}{R_{A} R_{B} R_{A B}}\left(\hat{\mathbf{R}}_{A} \times \hat{\mathbf{R}}_{B}\right) \cdot\left(\mathbf{S}_{A} \times \mathbf{S}_{B}\right)
$$

no qual $R_{A B} \equiv\left|\mathbf{R}_{A}-\mathbf{R}_{B}\right|$, e $k_{F}$ simboliza o módulo do vetor de onda de Fermi. O mecanismo expresso na Eq. A.2 depende explicitamente da separação entre os sítios $\mathbf{R}_{A}$ e $\mathbf{R}_{B}$; logo, é possível, a priori, caracterizá-lo como uma interação do tipo RKKY. Porém, seu caráter antissimétrico segue o termo proposto pela Eq. 2.12, e sugere que este acoplamento manifesta a mesma natureza descrita por Dzyaloshinskii e Moriya responsável, assim, pelo aumento no campo anisotrópico do material.

Apesar de apresentarmos aqui a interação de DM como efeito do spin-órbita, resultados teóricos recentes [163] têm demonstrado a existência de um acoplamento de DM de origem não-relativística - e que é responsável (em conjunto com outros fatores) pelo magnetismo do composto $\mathrm{Mn}_{3} \mathrm{Sn}$. 


\section{Apêndice B}

\section{Formalismo LMTO-ASA}

Neste apêndice, apresentaremos o formalismo no espaço recíproco LMTO-ASA [265]. Em um primeiro momento, o LMTO-ASA foi desenvolvido na chamada base canônica. Posteriormente, Olle K. Andersen [131] demonstrou que o mesmo formalismo poderia ser descrito em termos de outras bases. Assim, cria-se a liberdade de escolha para a base mais apropriada em cada caso. Para os sistemas que estamos interessados, com dimensionalidade reduzida, há duas bases de maior interesse, a saber: a ortogonal, na qual assume-se a ortogonalidade entre as funções de onda, e a base tight-binding (mais localizada), obtida de maneira a se considerar as interações entre os sítios vizinhos com o menor alcance possível.

O apêndice é dividido em duas principais partes. Na primeira (seção B.1) descrevemos o que são os orbitais lineares do tipo muffin-tin (LMTO), e o formalismo LMTO-ASA no espaço k, descrito em termos de diferentes bases. Já na segunda (seção B.2), desenvolvemos o Método de Recorrência, que permite evidenciar o caráter local da Hamiltoniana do sistema, e, a partir disto, obter resultados característicos do material como um todo.

\section{B.1 Orbitais lineares do tipo muffin-tin (LMTO) e o formalismo LMTO-ASA no espaço $k$}

Na presente seção são descritas as diferentes bases das quais o LMTO-ASA se utiliza: a base canônica, genérica, ortogonal e localizada (tight-binding, TB). Conforme será discutido mais à frente, estas duas últimas caracterizam-se como bases de maior interesse para os sistemas investigados neste trabalho. A base ortogonal, como a própria nomenclatura 
sugere, envolve funções ortonormais entre si, enquanto a base TB é obtida visando o menor alcance possível das interações entre sítios vizinhos. O LMTO-ASA é um formalismo extensamente explorado na literatura [266], e, portanto, aqui sua exposição tem caráter introdutório (e não deve substituir a já bem consolidada bibliografia nesta área). O desenvolvimento desta seção teórico está fundamentado nos anais da International School of Physics "Enrico Fermi", de 1985, elaborados por Andersen, Jepsen e Glötzel [131]; um detalhamento maior das passagens aqui apresentadas pode ser encontrado, por exemplo, nas referências [131, 137, 138].

\section{B.1.1 Matrizes Hamiltoniana $(\mathcal{H})$ e de overlap (O)}

A solução da Eq. 3.6 pode ser facilmente atingida se a $j$-ésima autofunção $\psi_{j}(\mathbf{r}) \equiv$ $\psi_{j}(\mathbf{r}, E)$ de $\mathcal{H}$ for expressa segundo a expansão [125]:

$$
\psi_{j}(\mathbf{r}, E)=\sum_{i} \chi_{R L, i}(\mathbf{r}) u_{R L, i}(E)
$$

sobre para um conjunto finito de $i$ funções de base $\left\{\chi_{R L}\right\}$, independentes da energia. Isto porque, caso a base seja dependente da energia é necessário levar em conta um enorme intervalo de energias $E$ para se obter soluções para a Eq. 3.6 com precisão aceitável, diminuindo a eficiência do método. Na Eq. B.1, cada $u_{R L, i}$ e $\chi_{R L, i}$ é calculado para um determinado sítio $\mathbf{R}$ da rede cristalina e um valor de momento angular $L=\{l, m\}$ conhecido. Ao contrário das funções $\left\{\chi_{R L}\right\}$, os coeficientes $\left\{u_{R L}\right\}$ da expansão de $\psi_{j}(\mathbf{r})$ são dependentes da energia, e, com o auxílio do princípio variacional de Rayleigh-Ritz [267], podem ser determinados como os autovetores do seguinte problema de autovalores (Eq. 3.6 na representação matricial $)^{1}$ :

$$
(\mathcal{H}-E \mathbf{O}) \mathbf{u}=\mathbb{O}
$$

em que $\mathcal{H} \equiv\left(\mathcal{H}_{R L, R^{\prime} L^{\prime}}\right)$ é a matriz Hamiltoniana, $\mathbf{O} \equiv\left(O_{R L, R^{\prime} L^{\prime}}\right)$ é a matriz de overlap, e $E$ representa a autoenergias. Assim, os elementos de $\mathcal{H}$ e de $\mathbf{O}$ são consequentemente definidos por:

$$
\mathcal{H}_{R L, R^{\prime} L^{\prime}}=\left\langle\chi_{R^{\prime} L^{\prime}}|\mathcal{H}| \chi_{R L}\right\rangle \equiv\left\langle\chi_{R^{\prime} L^{\prime}}\left|-\nabla^{2}+V_{\mathrm{eff}(\mathbf{r})}\right| \chi_{R L}\right\rangle
$$

\footnotetext{
${ }^{1}$ Ao longo do desenvolvimento teórico deste capítulo, quando a equação assim o exigir, E será tratado como uma matriz diagonal com os autovalores de energia. Caso contrário, E será o próprio valor da energia.
} 
e

$$
O_{R L, R^{\prime} L^{\prime}}=\left\langle\chi_{R^{\prime} L^{\prime}} \mid \chi_{R L}\right\rangle
$$

Almeja-se encontrar uma teoria que fundamente e possibilite a utilização de um método no espaço real de forma controlada. Logo, torna-se necessário que um conjunto de base fixo $\left\{\chi_{R L}\right\} \equiv\{\chi\}$ seja, ao mesmo tempo, minimo (1 orbital $s, 3$ orbitais $p$, e 5 orbitais d, no máximo, por sítio), localizado e ortonormal. A fim de satisfazer estas condições, deseja-se que as matrizes $\mathcal{H}$ e $\mathbf{O}$ tenham as mínimas dimensões possíveis e sejam de curto alcance, exigência matematicamente satisfeita por [232]:

$$
\begin{cases}\mathcal{H}_{R L, R^{\prime} L^{\prime}} \sim 0, & \text { se }\left|\mathbf{R}-\mathbf{R}^{\prime}\right|>R_{\text {máx }} \\ O_{R L, R^{\prime} L^{\prime}} \sim 0, & \text { se }\left|\mathbf{R}-\mathbf{R}^{\prime}\right|>R_{\text {máx }}\end{cases}
$$

onde $\mathbf{R}$ e $\mathbf{R}^{\prime}$ são sítios atômicos no cristal.

Candidatos naturais para este método de base fixa, as funções atômicas, não fornecem uma descrição conveniente. Assim, o formalismo LMTO foi primeiramente desenvolvido na chamada base canônica. Posteriormente, como já mencionado na introdução, Andersen [131] demonstrou que o LMTO também pode ser desenvolvido em termos de outras bases, criando-se a liberdade de escolha para a base que seja mais apropriada ao tratamento do sistema de interesse.

\section{B.1.2 Potenciais do tipo muffin-tin}

Tal como mencionado anteriormente, a escolha do conjunto de funções de base $\left\{\chi_{R L}\right\}$ deve ser feita de maneira conveniente para que se resulte em uma matriz Hamiltoniana (Eq. B.3) e uma matriz de ouverlap (Eq. B.4) com as mínimas dimensões possíveis. Isto é, espera-se obter um conjunto $\left\{\chi_{R L}\right\}$ mínimo no qual apenas um orbital por valor de momento angular $L$ seja suficiente para uma boa descrição das propriedades do sistema em questão. No caso dos metais de transição, deve-se considerar, então, 9 orbitais por sítio, a saber: $s, p_{x}, p_{y}, p_{z}, d_{x y}, d_{x z}, d_{y z}, d_{x^{2}-y^{2}}$, e $d_{3 z^{2}-r^{2}}$.

Um dos conjuntos de base existentes para o cálculo de estrutura eletrônica de primeiros princípios é constituído dos orbitais muffin-tin (MTO) [125, 131]. Nele, cada átomo do cristal é representado por uma esfera de raio $s$ ao redor do sítio cristalino $\mathbf{R}$ e, portanto, seu potencial $V_{M T}(\mathbf{r})$ é esfericamente simétrico. Por outro lado, fora desta esfera (região intersticial) considera-se que o potencial muffin-tin assume um valor constante, denotado 
aqui por $V_{0}$. Assim, convém por simplicidade ajustar $V_{M T}(\mathbf{r})$ de tal maneira que seu valor na região $r>s$ seja nulo, isto é:

$$
V_{M T}(\mathbf{r})= \begin{cases}V(r)-V_{0}, & \text { se } r \leq s \\ 0, & \text { se } r>s\end{cases}
$$

onde $r \equiv|\mathbf{r}-\mathbf{R}|$

Um potencial $V_{\text {eff }}(\mathbf{r})$ típico é mostrado na Fig. B.1, obtida para uma posição próxima à superfície (100) do Ni cristalino fcc. Particularmente, na Fig. B.1 (b) apresenta-se um potencial do tipo muffin-tin, em que $V_{\text {eff }}(\mathbf{r})=V_{M T}(\mathbf{r})$ (Eq. B.6). Nota-se uma clara semelhança entre as funções apresentadas nas Figs. B.1(a) e B.1(b), o que caracteriza o potencial do tipo muffin-tin (seção B.1) como uma boa aproximação.

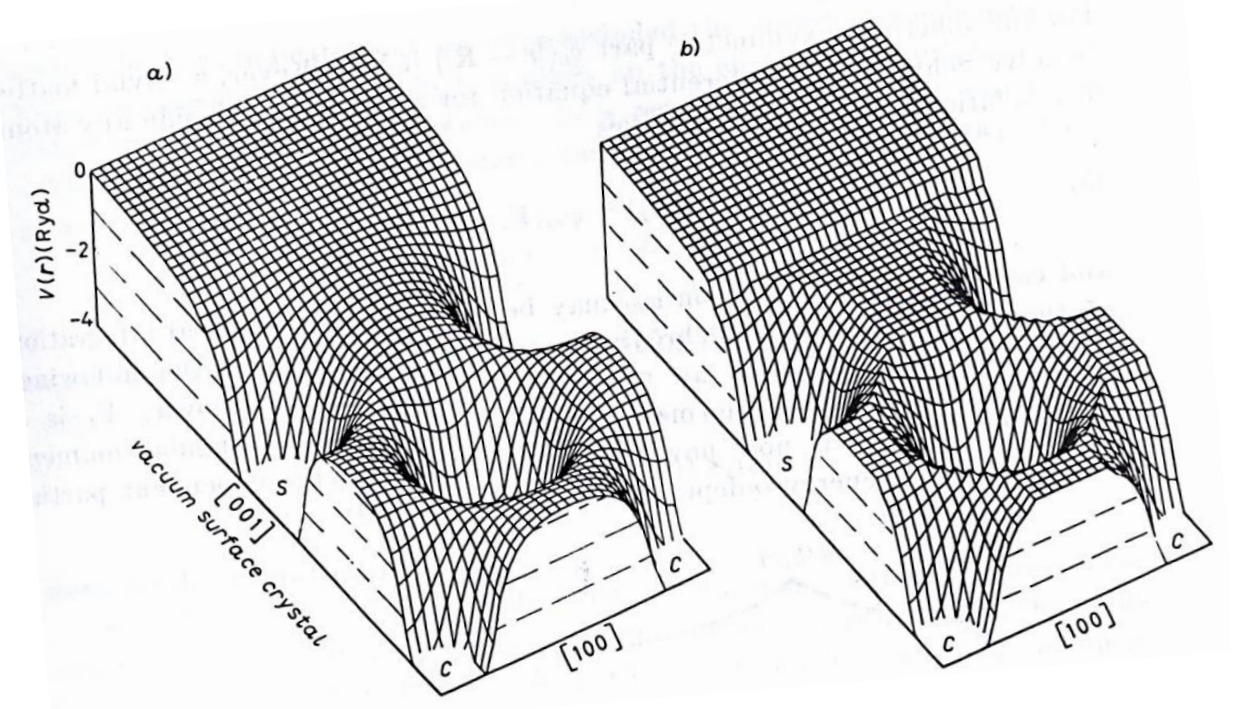

Figura B.1: Potencial monoeletrônico $V_{\mathrm{eff}}(\mathbf{r})$ autoconsistente obtido próximo à superfície de (100) do Ni bulk fcc, por meio do método LAPW [131]: (a) full-potential; (b) potencial muffin-tin (aproximado).

A escolha do potencial apresentado na Eq. B.6 visa, sobretudo, facilitar o casamento dos orbitais de um sítio a outro, assumindo que os elétrons propagam-se livremente entre as esferas existentes em um dado cristal com um número de onda constante $\kappa=\sqrt{E-V_{0}}$, sendo $E$ uma autoenegia da Eq. B.2. A partir desta definição, a simetria esférica se estende por todo o espaço. Logo, as funções $\psi_{j}(\mathbf{r}, E)$ podem ser separadas em produtos de uma parte radial $\left(\varphi_{R l}(r, E)\right)$ e outra harmônica $\left(Y_{L}(\hat{\mathbf{r}})\right)$, na qual $\hat{\mathbf{r}} \equiv f(\theta, \phi)=(\cos (\theta) \operatorname{sen}(\phi), \operatorname{sen}(\theta) \operatorname{sen}(\phi), \cos (\phi))$. Dessa forma, $\left\{\chi_{R L}\right\}$ pode ser obtido pela solução da Eq. 3.6 considerando-se $V_{\text {eff }}(\mathbf{r})=V_{M T}(\mathbf{r})-\kappa^{2}$. Obviamente, a parte radial da solução dentro da esfera dependerá da solução na região fora da mesma por 
meio da imposição de condições de continuidade e diferenciabilidade na borda $r=s$. A parte radial, $\varphi_{R l}(r, E)$, deve ser regular na origem (especificamente $\varphi \sim r^{l}$ ) para que seja normalizável, e é obtida pela integração numérica da seguinte equação de Schrödinger, no âmbito do formalismo de KS:

$$
\left[-\frac{d^{2}}{d r^{2}}+\frac{l(l+1)}{r^{2}}+V_{M T}(\mathbf{r})-\kappa^{2}\right] r \varphi_{R l}(r, E)=0(r \leq s)
$$

Já na região fora da esfera, por sua vez, o potencial constante reduz a Eq. 3.6 à bem conhecida equação de Helmholtz:

$$
\left[-\frac{d^{2}}{d r^{2}}-\kappa^{2}\right] r \varphi_{R l}(r, E)=0(r>s)
$$

para a qual existem duas soluções linearmente independentes: as funções esféricas de Bessel $j_{R l}(\kappa r)$ e de Neumann $k_{R l}(\kappa r)$, respectivamente definidas no limite de pequenos valores de $\kappa r$ (i.e., $\kappa r \rightarrow 0$ ) por [125]:

$$
\left\{\begin{array}{l}
j_{R l}(\kappa r) \rightarrow \frac{(\kappa r)^{l}}{(2 l+1) ! !} \\
k_{R l}(\kappa r) \rightarrow-\frac{(2 l-1) ! !}{(\kappa r)^{l+1}}
\end{array}\right.
$$

em termos do duplo fatorial (!!), caracterizado para qualquer número $n$ natural por $n ! !=$ $n(n-2)(n-4) \ldots$, e $(-1) ! !=1$. Quando $\kappa r \rightarrow \infty$, as funções $j_{R l}(\kappa r)$ e $k_{R l}(\kappa r)$ assumem as seguintes formas assintóticas [125]:

$$
\left\{\begin{array}{l}
j_{R l}(\kappa r) \rightarrow \frac{\operatorname{sen}\left(\kappa r-\frac{l \pi}{2}\right)}{\kappa r} \\
k_{R l}(\kappa r)=\rightarrow-\frac{\cos \left(\kappa r-\frac{l \pi}{2}\right)}{\kappa r}
\end{array}\right.
$$

ambas regulares no infinito. Pela Eq. B.9, no entanto, apenas $j_{R l}(\kappa r)$ mostra-se regular na origem. Tendo em vista que as funções de base $\left\{\chi_{R L}\right\}$ devem seguir os requisitos apresentados anteriormente, Andersen [265] introduziu os chamados orbitais muffin-tin adicionando uma função de Bessel esférica à solução radial $\varphi_{R l}(r, E)$ dentro da esfera localizada no sítio $\mathbf{R}$, com o objetivo de cancelar a parte divergente de $\varphi_{R l}(r, E)$, além de reduzir a dependência da energia nas caudas (ou seja, em $r>s$ ). Assim, tratamse de funções aproximadamente independentes da energia, razoavelmente localizadas e normalizáveis para todos os valores de $\kappa^{2}$. De modo explícito, as funções de base MTO 
$\chi_{R L, i}$ de Andersen são descritas por:

$$
\chi_{R L, i}(E, \kappa, \mathbf{r})=Y_{L}(\hat{\mathbf{r}}) \begin{cases}\varphi_{R l}(r, E)+\kappa \operatorname{cotg}\left(k_{R l}\right) j_{R l}(\kappa r), & \text { se } r \leq s \\ \kappa k_{R l}(\kappa r), & \text { se } r>s\end{cases}
$$

Uma propriedade importante da Eq. B.11 é que a função definida em $r \leq s$ é regular na origem, enquanto a cauda $\kappa k_{R l}(\kappa r)$ é regular no infinito. O fato de $k_{R l}(\kappa r)$ divergir na origem (pois $l \geq 0$ ) não é relevante, visto que ela servirá apenas para estabelecer o comportamento da base canônica no contorno das esferas. De fato, como será detalhado adiante, as funções esféricas de Neumann atuarão como funções envelope na região $r=s$ quando assumida a aproximação ASA. É necessário também destacar que a Eq. B.11 não é totalmente independente da energia, pois carrega a autoenergia $E$ na variável $\kappa$. Entretanto, é possível suprimir esta dependência fixando-se um valor adequado de $\kappa$, que resulta em uma energia particular $E=E_{\nu}$. Como ficará demonstrado mais adiante, esta técnica permite fazer a aproximação básica de um método linear, que é a substituição função $\varphi_{R l}(r, E)$ (dependente da energia) pelos dois primeiros termos de sua expansão em série de Taylor em torno de $E_{\nu}$. Constroem-se, então, os MTO linearizados (LMTO) [232]. Matematicamente, isto equivale fazer a seguinte aproximação para $\varphi_{R l}(r, E)$ em $r \leq s:$

$$
\varphi_{R l}(r, E) \sim \varphi_{R l}\left(r, E_{\nu}\right)+\left(E-E_{\nu}\right) \dot{\varphi}_{R l}\left(r, E_{\nu}\right)
$$

na qual $\dot{\varphi}$ é a primeira derivada em relação à energia de $\varphi\left(\dot{\varphi}_{R l}\left(r, E_{\nu}\right)=\left.\frac{\partial}{\partial E} \varphi_{R l}(r, E)\right|_{E=E_{\nu}}\right)$. Em outras palavras, a construção das funções de base $\chi_{R L}(\mathbf{r})$ abrangerá essencialmente combinações lineares de $\varphi$ e $\dot{\varphi}$. De fato, se $E-E_{\nu} \leq 0,1$ Ry, o próximo termo da expansão de Taylor, $\frac{1}{2}\left(E-E_{\nu}\right)^{2} \ddot{\varphi}$, é duas ou três ordens de grandeza menor que $\varphi$, justificando, assim, a aproximação da Eq. B.12 de acordo com a escolha de $E_{\nu}$. As funções $\varphi_{R l}(r, E)$ e $\dot{\varphi}_{R l}(r, E)$ são, então, normalizadas em suas próprias esferas $\left(\int_{0}^{s} \varphi_{R l}(r, E) r^{2} d r=1\right)$ e construídas de forma a respeitar a ortogonalidade entre $\varphi$ e $\dot{\varphi}\left(\int_{0}^{s} \varphi_{R l}(r, E) \dot{\varphi}_{R l}(r, E) r^{2} d r=0\right)$ [137].

Por simplicidade, consideraremos aqui um arranjo cristalino como uma associação de células primitivas contendo um único átomo. O espaço é, então, dividido em esferas muffin-tin centradas nos átomos constituintes e em regiões intersticiais (ou interstícios). A Fig. B.2 ilustra com precisão esta definição. Nesse caso, o termo $k_{R l}(\kappa r)$ na Eq. B.11, relacionado com a função centrada no sítio $\mathbf{R}$, é expandido em termos das funções de Bessel $j_{R^{\prime} L^{\prime}}(\kappa \mathbf{R})=j_{R^{\prime} l^{\prime}}(\kappa r) Y_{L^{\prime}}(\hat{\mathbf{r}})$ dentro das demais esferas, centradas em $\mathbf{R}^{\prime}$. Este processo, entretanto, será desenvolvida dentro da aproximação ASA, na qual desconsideram-se os interstícios. 


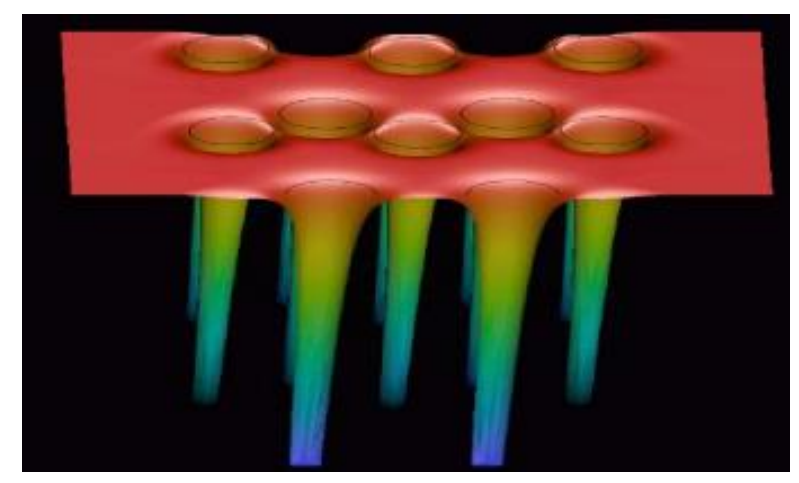

Figura B.2: Potencial muffin-tin cristalino obtido como resultado de um cálculo utilizando o método FLAPW [268]. A região intersticial é identificada em vermelho, enquanto as funções $V_{M T}(\mathbf{r})$ dentro das esferas centradas nos átomos do material são representadas pelos poços em cores frias.

Apresentados de maneira introdutória os orbitais do tipo muffin-tin, convém seguir com a aproximação ASA. Nela, o número de onda será fixado em $\kappa^{2}=0$.

\section{B.1.3 Aproximação da esfera atômica (ASA)}

Junto à definição dos MTO são usadas algumas aproximações que visam simplificar ainda mais os cálculos de estrutura eletrônica. A principal delas é a ASA, na qual um cristal é considerado totalmente preenchido por esferas centradas em cada átomo do material, de modo que as regiões intersticiais e de sobreposição (overlaps) são desprezadas. A rigor, a aproximação ASA trata-se de um termo cunhado por Andersen [131] que consiste essencialmente em duas aproximações: $(i)$ o valor de $\kappa^{2}$ é fixado em um valor adequado (na prática, $\kappa^{2}=0$ ), tornando-o independente da energia $E$; $(i i)$ a célula de Wigner-Seitz (WS) do material é reduzida a uma esfera de mesmo volume $V_{\Omega}$ desta célula, com raio $r_{W S}$. Assim, pela proposição $(i i)$, o raio de cada esfera muffin-tin é tal que $r_{W S}=\left(\frac{3 V_{\Omega}}{4 \pi}\right)^{\frac{1}{3}}$. A ASA é conveniente na presente investigação, pois este trabalho tratará de sistemas densamente empacotados. No caso de um material formado por mais de um tipo de átomo, deve-se satisfazer a relação $\frac{4 \pi}{3} \sum_{R} r_{W S_{R}}^{3}=V_{\Omega}$, onde $r_{W S_{R}}$ é o raio de WS de um dado átomo em $\mathbf{R}$, e o somatório $\sum_{R}$ se estende sobre todos os sítios contidos na célula primitiva. Por exemplo, para uma liga binária $A B$, as esferas que circundam o átomo da espécie $A$ podem ter um raio proporcional ao de WS do $A$ puro, enquanto as esferas que circundam o átomo $B$ podem ter o raio proporcional ao de WS do elemento $B$ puro, sendo que ambos devem ser ajustados para que a soma dos volumes seja igual a $V_{\Omega}$.

A escolha de $\kappa^{2}=0$ produz consequências interessantes. Neste caso, as funções 
esféricas de Bessel e Neumann seguem as formas definidas na Eq. B.9 para $\kappa r \rightarrow 0$. Se $a$ é um fator de escala arbitrário (raio médio de WS, parâmetro de rede do material investigado, entre outros), determinado de maneira a tornar as funções $j_{R l}(\kappa r) \equiv j_{R l}(r)$ e $K_{R l}(\kappa r) \equiv k_{R l}(r)$ adimensionais, então a Eq. B.9 pode ser reescrita do seguinte modo $[125,138]$ :

$$
\left\{\begin{array}{l}
j_{R l}(r)=\frac{1}{2(2 l+1)}\left(\frac{r}{a}\right)^{l} \\
k_{R l}(r)=\left(\frac{a}{r}\right)^{l+1}
\end{array}\right.
$$

onde $k_{R l}(r)$ é, por exemplo, a solução da equação de Laplace $\nabla^{2} k_{R l}(r)=0$, regular no infinito, e irregular na origem. Frequentemente, adota-se a como sendo o raio de WS do material [138]. Por simplicidade de notação, e tendo como base as variáveis definidas anteriormente, indicaremos, de agora em diante, esse raio por $s$. Note que $j_{R l}(r)$ e $k_{R l}(r)$ não dependem mais do número de onda $\kappa$, pois, fixado, este se torna apenas um parâmetro no problema em questão. Usando a condição que para $r \rightarrow \infty$ a função de base $\chi_{R L, i}$ deve se anular, então a região intersticial é descrita apenas por $k_{R l}(r)$ :

$$
\chi_{R L, i}(r)=\left(\frac{s}{r}\right)^{l+1} Y_{L}(\hat{\mathbf{r}}), \text { se } r>s
$$

em semelhança ao descrito na Eq. B.11, mas envolvendo a forma explícita da função de Neumann apresentada na Eq. B.13. O fato de se assumir $\chi_{R L, i}$ nulo no infinito está relacionado à necessidade de construção da base mais localizada possível. Na aproximação ASA, $K_{R L}(\mathbf{r})=k_{R l}(r) Y_{L}(\hat{\mathbf{r}})$ atua como uma função envelope, isto é, que descreve o comportamento da base na borda das esferas $(r=s)$ (veja a Fig. B.3, abaixo). Vale a pena também destacar que esta função envelope possui a mesma forma que um potencial eletrostático de um multipolo pontual [232].

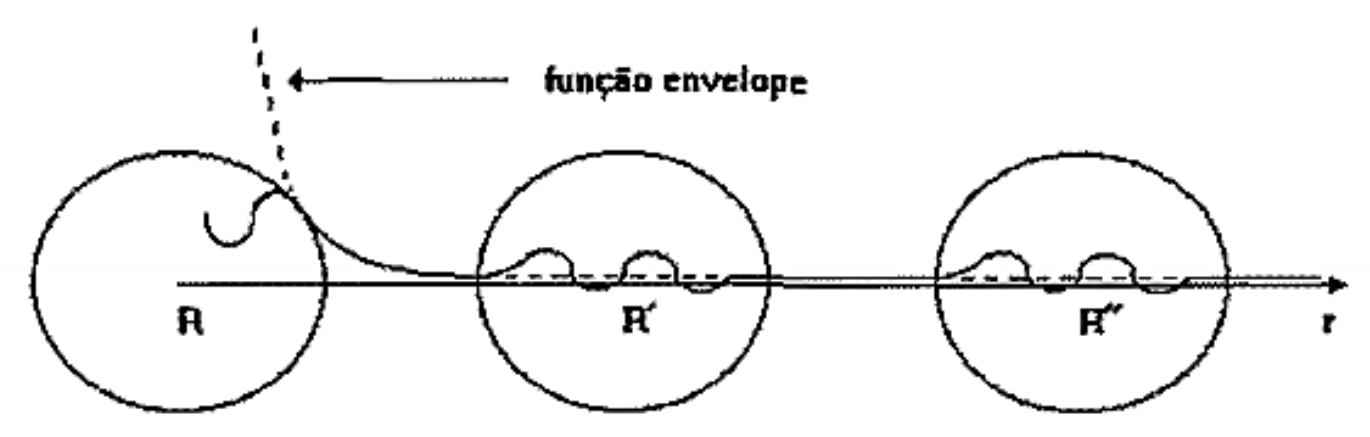

Figura B.3: Representação esquemática da função envelope na esfera em R. Figura reproduzida da referência [137].

Ambas as soluções nas regiões fora e dentro da esfera localizada em $\mathbf{R}$ podem ser 
combinadas suavemente na borda para gerar uma nova definição de MTO, respeitando-se as condições de continuidade:

$$
\chi_{R L, i}(r, E)= \begin{cases}\mathcal{W}\left(\varphi_{\nu}, \dot{\varphi}_{\nu}\right)_{R L}^{-1}\left[\mathcal{W}\left(K, \dot{\varphi}_{\nu}\right)_{R L} \varphi_{R L \nu}-\mathcal{W}\left(K, \varphi_{\nu}\right)_{R L} \dot{\varphi}_{R L \nu}\right], & \text { se } r \leq s \\ K_{R L}, & \text { se } r>s\end{cases}
$$

onde $\varphi_{R L \nu} \equiv \varphi_{R l}\left(r, E_{\nu}\right) Y_{L}(\hat{\mathbf{r}}), \dot{\varphi}_{R L \nu} \equiv \dot{\varphi}_{R l}\left(r, E_{\nu}\right) Y_{L}(\hat{\mathbf{r}})$, e $\mathcal{W}$ representa o Wronskiano, calculado em $r=s$, que garante a condição de continuidade necessária. Assim, se $f(r)$ e $g(r)$ são duas funções deriváveis, o Wronskiano no ponto de interesse $r=s$ é definido por:

$$
\mathcal{W}(f, g)=s^{2}\left|\begin{array}{cc}
f(s) & g(s) \\
\left.\frac{\partial}{\partial r} f(r)\right|_{r=s} & \left.\frac{\partial}{\partial r} g(r)\right|_{r=s}
\end{array}\right|=s^{2}\left[f(r) g^{\prime}(r)-f^{\prime}(r) g(r)\right]
$$

Neste caso, é importante lembrar que, se $\kappa^{2}=0$ é uma quantidade fixa, então as equações diferenciais B.7 e B.8 modificam-se, e suas soluções não serão as mesmas da Eq. B.11 - resolvida para o caso geral. Isto significa que a notação baseada em $\operatorname{cotg}\left(k_{R l}\right)$ (phase-shift) não tem mais sentido [125], passando a valer como definição do MTO a Eq. B.15.

Finalmente, na ASA utilizam-se apenas os orbitais $s, p, d$ (e $f$ ). Portanto, as matrizes Hamiltoniana e de overlap (seção B.1.1) são escritas levando-se em conta que $\kappa^{2}=0$, sendo truncadas até $l_{\text {máx }}=2$ ou $l_{\text {máx }}=3$ - escolha que depende do sistema investigado [132]. Apresentadas a aproximação da esfera atômica e os orbitais muffin-tin linearizados (Eq. B.12), é natural, portanto, seguir com a definição das bases canônica, genérica, ortogonal, e TB, com as quais se desenvolveu o método que considera estas duas abordagens simultaneamente: o LMTO-ASA [130, 131].

\section{B.1.4 Obtenção da base canônica $\left\{\chi_{R L}^{0}\right\}$}

Nesta seção, emprega-se o sobrescrito 0 (zero) a fim de diferenciar a base canônica de outras bases utilizadas no LMTO-ASA. A construção de $\left\{\chi_{R L}^{0}\right\}$ inicia-se com a definição explícita da função envelope relacionada à esfera muffin-tin em $\mathbf{R}$, introduzida na seção anterior. Tal como mencionado, seu papel é estabelecer as condições de continuidade no contorno $r=s$. No caso da base canônica, a função envelope no sítio $\mathbf{R}$ é a própria $K_{R L}(\mathbf{r}) \equiv K_{R L}^{0}(\mathbf{r})$, reescrita como um produto entre a função esférica de Neumann $\left(k_{R l}(r) \equiv k_{R l}^{0}(r)\right)$ no limite de $\kappa r$ pequeno, e do esférico harmônico $Y_{L}(\hat{\mathbf{r}})$ (tal como 
descrito pela Eq. B.14):

$$
K_{R L}^{0}(\mathbf{r})=k_{R l}^{0}(r) Y_{L}(\hat{\mathbf{r}})=\left(\frac{s}{r}\right)^{l+1} Y_{L}(\hat{\mathbf{r}}), \text { se } r>s
$$

A partir da Eq. B.17, prossegue-se com um procedimento comumente referido na literatura como augmentation [232], que consiste essencialmente de duas etapas. Resumidamente, o augmentation caracteriza-se como o processo de substituição da função envelope (Eq. B.17) vinculada a um único sítio, objetivando considerar os demais sítios (átomos) não-equivalentes do cristal. Note que, neste contexto, pretende-se calcular a estrutura eletrônica de um sistema cristalino, e não de um átomo isolado. Assim, a primeira etapa do augmentation é definida explicitamente pela expansão da parte de $K_{R L}^{0}(\mathbf{r})$ que se estende às demais esferas centradas nos sítios genéricos $\mathbf{R}^{\prime} \neq \mathbf{R}$ (cauda), denotada aqui por $K_{R^{\prime} L^{\prime}}^{0}\left(\mathbf{r}^{\prime}\right)$, em termos das soluções da equação de Laplace regulares na origem com momento angular $L^{\prime}=\left\{l^{\prime}, m^{\prime}\right\}$, haja vista que $k_{R^{\prime} l^{\prime}}^{0}\left(r^{\prime}\right)$ diverge no ponto $\mathbf{r}^{\prime}=0$. Em conformidade com a notação usada na Eq. B.9, o termo regular na origem será aqui representado por $j_{R^{\prime} l^{\prime}}^{0}\left(r^{\prime}\right)$; assim, por definição, $\nabla^{2} j_{R^{\prime} l^{\prime}}^{0}\left(r^{\prime}\right)=0$. A função $K_{R^{\prime} L^{\prime}}^{0}\left(\mathbf{r}^{\prime}\right)$ é, então, reescrita pela seguinte expansão com coeficientes $S_{R^{\prime} L^{\prime}, R L}^{0}$ :

$$
K_{R^{\prime} L^{\prime}}^{0}\left(\mathbf{r}^{\prime}\right)=-\sum_{R^{\prime} L^{\prime}} J_{R^{\prime} L^{\prime}}^{0}\left(\mathbf{r}^{\prime}\right) S_{R^{\prime} L^{\prime}, R L}^{0}
$$

na qual $r^{\prime}=\left|\mathbf{r}^{\prime}-\mathbf{R}^{\prime}\right|$, e as funções $J_{R^{\prime} L^{\prime}}^{0}\left(r^{\prime}\right)$ são dadas por:

$$
J_{R^{\prime} L^{\prime}}^{0}\left(\mathbf{r}^{\prime}\right)= \begin{cases}j_{R^{\prime} l^{\prime}}^{0}(r) Y_{L^{\prime}}\left(\hat{\mathbf{r}}^{\prime}\right)=\frac{1}{2\left(2 l^{\prime}+1\right)}\left|\frac{r^{\prime}}{s}\right|^{l^{\prime}} Y_{L^{\prime}}\left(\hat{\mathbf{r}}^{\prime}\right), & \text { se } r^{\prime} \leq s \\ 0, & \text { caso contrário }\end{cases}
$$

Na Eq. B.18, os coeficientes $S_{R^{\prime} L^{\prime}, R L}^{0}$ dependem apenas das distâncias entre os sítios $\mathbf{R}$ e $\mathbf{R}^{\prime}$, anulando-se para $\mathbf{R}=\mathbf{R}^{\prime}$. Além disso, o fator $\frac{1}{2\left(2 l^{\prime}+1\right)}$ produz uma matriz $\mathbf{S}^{0} \equiv\left(S_{R^{\prime} L^{\prime}, R L}^{0}\right)$ hermitiana. Assim, por depender da organização espacial característica do material estudado, $\mathbf{S}^{0}$ é denominada matriz de estrutura canônica [265]. Seus elementos $S_{R^{\prime} L^{\prime}, R L}^{0}$ são determinados pela expressão:

$$
S_{R^{\prime} L^{\prime}, R L}^{0}=\frac{1}{\sqrt{4 \pi}} G_{l^{\prime} m^{\prime}, l m}\left|\frac{\mathbf{R}-\mathbf{R}^{\prime}}{s}\right|^{-l^{\prime}-l-1} Y_{l^{\prime}+1, m^{\prime}-m}^{*}\left(\frac{\mathbf{R}-\mathbf{R}^{\prime}}{\left|\mathbf{R}-\mathbf{R}^{\prime}\right|}\right)
$$


na qual os parâmetros $G_{l^{\prime} m^{\prime}, l m}$ valem:

$$
G_{l^{\prime} m^{\prime}, l m}=(-1)^{l+m+1} \sqrt{\frac{\left(2 l^{\prime}+1\right)(2 l+1)\left(l+l^{\prime}+m^{\prime}-m\right) !\left(l+l^{\prime}-m^{\prime}+m\right) !}{\left(2 l^{\prime}+2 l+1\right)\left(l^{\prime}+m^{\prime}\right) !\left(l^{\prime}-m^{\prime}\right) !(l+m) !(l-m) !}} .
$$

Os valores de $S_{R^{\prime} L^{\prime}, R L}^{0}$ e $G_{l^{\prime} m^{\prime}, l m}$ apresentados nas Eqs. B.20 e B.21 são obtidos por meio do Teorema da Expansão a que as funções $j_{R l}^{0}(r)$ e $k_{R l}^{0}(r)$ estão sujeitas; $G_{l^{\prime} m^{\prime}, l m}$ caracterizam-se, portanto, como os coeficientes desta expansão em $l^{\prime}$. Um desenvolvimento detalhado desta passagem pode ser encontrado na referência [125]. Assim, a função envelope final, associada a todo o espaço externo às esferas e denotada aqui por $\left(K_{R L}^{0}\right)^{\infty}$, pode ser escrita em termos de $K_{R L}(\mathbf{r})$ centrada em $\mathbf{R}$ (Eq. B.17) e da Eq. B.18 como:

$$
\begin{aligned}
& \left(K_{R L}^{0}\right)^{\infty}=K_{R L}^{0}(\mathbf{r})-\sum_{R^{\prime} L^{\prime}} J_{R^{\prime} L^{\prime}}^{0}\left(\mathbf{r}^{\prime}\right) S_{R^{\prime} L^{\prime}, R L}^{0} \\
= & k_{R l}^{0}(r) Y_{L}(\hat{\mathbf{r}})-\sum_{R^{\prime} L^{\prime}} j_{R^{\prime} l^{\prime}}^{0}\left(r^{\prime}\right) Y_{L^{\prime}}\left(\hat{\mathbf{r}}^{\prime}\right) S_{R^{\prime} L^{\prime}, R L}^{0} .
\end{aligned}
$$

Percebe-se que a função harmônica esférica $Y_{L^{\prime}}\left(\hat{\mathbf{r}}^{\prime}\right)$, como de costume, está implicitamente incluída em $J_{R^{\prime} L^{\prime}}^{0}\left(\mathbf{r}^{\prime}\right)$. Ainda por esta definição, é possível notar que $\left(K_{R L}^{0}\right)^{\infty}$ continua divergindo na origem do sítio $\mathbf{R}$, essencialmente por envolver a função $K_{R L}^{0}(\mathbf{r})$. De modo análogo à Eq. B.13), as funções esféricas $k_{R l}^{0}(r)$ e $j_{R^{\prime} l^{\prime}}^{0}\left(r^{\prime}\right)$ foram definidas por:

$$
k_{R l}^{0}(r)=\left|\frac{r}{s}\right|^{-l-1}
$$

e

$$
j_{R^{\prime} l^{\prime}}^{0}\left(r^{\prime}\right)=\frac{1}{2\left(2 l^{\prime}+1\right)}\left|\frac{r^{\prime}}{s}\right|^{l^{\prime}}
$$

É também possível expressar o conjunto de funções envelope de maneira simplificada, com o auxílio da notação bra-ket, ao considerar os termos da Eq. B.22 como componentes de vetores linha $|\ldots\rangle^{1}$ :

$$
\left|K^{0}\right\rangle^{\infty}=\left|K^{0}\right\rangle-\left|J^{0}\right\rangle \mathbf{S}^{0}
$$

onde, explicitamente, os vetores $\left|K^{0}\right\rangle$, $\left|J^{0}\right\rangle$ e $\left|K^{0}\right\rangle^{\infty}$ possuem as componentes $\left|K_{R L}^{0}\right\rangle$, $\left|J_{R^{\prime} L^{\prime}}^{0}\right\rangle$ e $\left|K_{R L}^{0}\right\rangle^{\infty}$, nesta ordem. De modo similar, $\langle K|,\left\langle J^{0}\right| \mathrm{e}^{\infty}\left\langle K^{0}\right|$ são vetores coluna (complexos conjugados), enquanto $\mathbf{S}^{0},{ }^{\infty}\left\langle K^{0} \mid K^{0}\right\rangle^{\infty}$, entre outros, são matrizes por cons-

\footnotetext{
${ }^{1}$ Note que esta notação não segue o padrão usual empregada para a descrição de autovetores em Mecânica Quântica. Assim, o desenvolvimento teórico aqui está baseado na notação utilizada originalmente por Andersen [131]. Lembre-se também que, neste contexto, $\langle\phi \mid \phi\rangle$ representa uma matriz, e é equivalente ao produto externo (outer product) $|\phi\rangle\langle\phi|$ na notação usual.
} 
trução. Vale lembrar que, nesta nova notação, os vetores incluem as partes harmônicas $Y_{L}(\hat{\mathbf{r}})$ e $Y_{L^{\prime}}\left(\hat{\mathbf{r}}^{\prime}\right)$.

Até agora, o augmentation focou-se na região externa às esferas não-equivalentes do material. Definida a função $\left(K_{R L}^{0}\right)^{\infty}$, a próxima e última etapa do augmentation consiste na substituição da parte da função envelope no interior das esferas por funções relacionadas às soluções radiais $\varphi_{R l}^{0}$ da Eq. B.7 nestas regiões (considerando $\kappa^{2}=0$, e, portanto, $\left.E=E_{\nu}\right)$. Seguindo a ideia de linearização esboçada na seção B.1.2, é possível escrever a base $\left(\chi_{R L}^{0}\right)^{\infty}$ independente da energia em todo o espaço interno às esferas muffin-tin em termos de uma combinação linear das partes radiais $\varphi_{R l}^{0}\left(r, E_{\nu}\right)$ e $\dot{\varphi}_{R l}^{0}\left(r, E_{\nu}\right)$ :

$$
\left(\chi_{R L}^{0}\right)^{\infty}=\varphi_{R l}^{0}\left(r, E_{\nu}\right) Y_{L}(\hat{\mathbf{r}})+\sum_{R^{\prime} L^{\prime}} \dot{\varphi}_{R^{\prime} l^{\prime}}^{0}\left(r^{\prime}, E_{\nu}\right) Y_{L^{\prime}}\left(\hat{\mathbf{r}}^{\prime}\right) h_{R^{\prime} L^{\prime}, R L}^{0}
$$

As funções radiais $\varphi_{R l}^{0}\left(r, E_{\nu}\right) \equiv \varphi_{R l}^{0}(r)$ e $\dot{\varphi}_{R l}^{0}\left(r, E_{\nu}\right) \equiv \dot{\varphi}_{R l}^{0}(r)^{1}$ são definidas como nulas nas regiões externas às esferas muffin-tin a que se referem. Além disso, pode-se mostrar que $\varphi_{R l}^{0}(r)$ e $\dot{\varphi}_{R l}^{0}(r)$ são aproximadamente ortogonais aos estados de caroço na esfera em $\mathbf{R}$ [137]. Em consequência, $\left(\chi_{R L}^{0}\right)^{\infty}$ apresenta a mesma propriedade. Assim, o processo de construção do orbital augmented (Eq. B.26) pode ser visto como uma forma de ortogonalizar os orbitais de base nos estados de caroço, e as autofunções $\psi_{j}$ (Eq. B.1) podem ser descritas em termos de um conjunto contendo um número limitado de funções de base [138]. Entretanto, nesta representação, $\varphi_{R l}^{0}(r)$ e $\dot{\varphi}_{R l}^{0}(r)$ não são necessariamente ortonormais. Na seção B.1.2, assumiu-se que $\varphi_{R l}(r, E)$ é normalizada à unidade $(\langle\varphi \mid \varphi\rangle=\mathbb{1})$, e também ortogonal à $\dot{\varphi}_{R l}(r, E)(\langle\varphi \mid \dot{\varphi}\rangle=\mathbb{O})$. A relação entre a onda parcial $\varphi_{R l}^{0}(r, E)$ dependente da energia e $\varphi_{R l}(r, E)$ é dada pelo seguinte [131, 232]

$$
\varphi_{R l}^{0}(r, E)=\left[1+\left(E-E_{\nu}\right) o_{R l}^{0}\right] \varphi_{R l}(r, E)
$$

válida, obviamente, para qualquer valor de $E$. Em particular, para $E=E_{\nu}$, tem-se que $\varphi_{R l}^{0}\left(r, E_{\nu}\right) \equiv \varphi_{R l}\left(r, E_{\nu}\right)$. Por outro lado, a partir da Eq. B.27 é possível também definir $\dot{\varphi}_{R l}^{0}\left(r, E_{\nu}\right)$ como função de $\varphi_{R l}\left(r, E_{\nu}\right)$ e $\dot{\varphi}_{R l}\left(r, E_{\nu}\right)$ como:

$$
\dot{\varphi}_{R l}^{0}\left(r, E_{\nu}\right)=\dot{\varphi}_{R l}\left(r, E_{\nu}\right)+\varphi_{R l}\left(r, E_{\nu}\right) o_{R l}^{0} .
$$

\footnotetext{
${ }^{1}$ Note que a dependência em energia pode ser omitida, pois $E=E_{\nu}$ representa apenas um parâmetro no problema.
} 


$$
\left(\chi_{R L}^{0}\right)^{\infty}=\varphi_{R l}\left(r, E_{\nu}\right) Y_{L}(\hat{\mathbf{r}})+\sum_{R^{\prime} l^{\prime}}\left[\dot{\varphi}_{R^{\prime} l^{\prime}}\left(r, E_{\nu}\right)+\varphi_{R^{\prime} l^{\prime}}\left(r, E_{\nu}\right) o_{R^{\prime} l^{\prime}, R^{\prime} l^{\prime}}^{0}\right] Y_{L^{\prime}}\left(\hat{\mathbf{r}}^{\prime}\right) h_{R^{\prime} L^{\prime}, R L}^{0}
$$

A Eq. B.28 revela que qualquer função derivada da energia é uma combinação linear do par ortonormal $\varphi_{R l}(r)$ e $\dot{\varphi}_{R l}(r)$. Finalmente, as matrizes $\mathbf{h}^{0}$ e $\mathbf{o}^{0}$, de elementos $h_{R^{\prime} L^{\prime}, R L}^{0}$ e $o_{R l}^{0}$, respectivamente, garantem a igualdade nas Eqs. B.28 e B.29, e são determinadas após a imposição das condições de contorno impostas pela função envelope $\left(K_{R L}\right)^{\infty}$; a forma explícita destas matrizes será derivada mais adiante. Utilizando novamente a notação vetorial, as expressões das Eqs. B.28 e B.29 ficam

$$
\begin{gathered}
\left|\dot{\varphi}^{0}\right\rangle=|\dot{\varphi}\rangle+|\varphi\rangle \mathbf{o}^{0}, \\
\left|\chi^{0}\right\rangle^{\infty}=|\varphi\rangle+\left|\dot{\varphi}^{0}\right\rangle \mathbf{h}^{0},
\end{gathered}
$$

onde $|\varphi\rangle$, por exemplo, é um vetor linha com componentes $\left|\varphi_{R L}\right\rangle$. As Eqs. B.30 e B.31 ser combinadas para gerar:

$$
\left|\chi^{0}\right\rangle^{\infty}=|\varphi\rangle\left(\mathbb{1}+\mathbf{o}^{0} \mathbf{h}^{0}\right)+|\dot{\varphi}\rangle \mathbf{h}^{0} .
$$

Neste caso $\mathbb{1}$ simboliza a matriz unitária de mesma dimensão que $\mathbf{o}^{0} \mathbf{h}^{0}$. Note que as partes angulares de $\varphi_{R L}(\mathbf{r}) \equiv \varphi_{R l}(r) Y_{L}(\hat{\mathbf{r}})$ e $\varphi_{R L}^{0}(\mathbf{r}) \equiv \varphi_{R l}^{0}(r) Y_{L}(\hat{\mathbf{r}})$ foram "absorvidas" nos vetores $|\ldots\rangle$. Além disso, assumiu-se que estes harmônicos esféricos estão normalizados à unidade em torno do sítio R. Por outro lado, se $\varphi_{R l}^{0}(r)$ está normalizada na esfera, haja vista sua equivalência com $\varphi_{R l}(r)$ pela Eq. B.28 se $E=E_{\nu}$, então:

$$
\left\langle\varphi^{0} \mid \varphi^{0}\right\rangle=\mathbb{1}=\langle\varphi \mid \varphi\rangle
$$

Da Eq. B.33 obtém-se por diferenciação com respeito à energia:

$$
\langle\dot{\varphi} \mid \varphi\rangle=\langle\varphi \mid \dot{\varphi}\rangle=\mathbb{0}
$$

$\mathrm{e}$

$$
\langle\dot{\varphi} \mid \dot{\varphi}\rangle=\mathbf{p}
$$

Na Eq. B.35 definiu-se a matriz diagonal p. As seguintes relações matriciais, que serão úteis posteriormente, também podem ser deduzidas fazendo-se o uso das relações 
$\left(-\nabla^{2}+V_{\text {eff }}(\mathbf{r})\right)|\varphi\rangle=E_{\nu}|\varphi\rangle$ e $\left(-\nabla^{2}+V_{\text {eff }}(\mathbf{r})\right)|\dot{\varphi}\rangle=|\varphi\rangle[131,232]$ (aqui $E_{\nu}$ é uma matriz diagonal com elementos $\left.E_{\nu R l}\right)$ :

$$
\begin{gathered}
\left\langle\varphi\left|-\nabla^{2}+V_{\text {eff }}(\mathbf{r})\right| \varphi\right\rangle=E_{\nu}, \\
\left\langle\dot{\varphi}\left|-\nabla^{2}+V_{\text {eff }}(\mathbf{r})\right| \varphi\right\rangle=\mathbb{O}, \\
\left\langle\varphi\left|-\nabla^{2}+V_{\text {eff }}(\mathbf{r})\right| \dot{\varphi}\right\rangle=\mathbb{1}, \\
\left\langle\dot{\varphi}^{0}\left|-\nabla^{2}+V_{\text {eff }}(\mathbf{r})\right| \varphi\right\rangle=E_{\nu} \mathbf{o}^{0}
\end{gathered}
$$

Da mesma forma, as seguintes expressões também podem escritas a partir da Eq. B.30:

$$
\begin{gathered}
\left\langle\varphi \mid \dot{\varphi}^{0}\right\rangle=\mathbf{o}^{0} \\
\left\langle\dot{\varphi}^{0} \mid \dot{\varphi}^{0}\right\rangle=\left(\mathbf{o}^{0}\right)^{2}+\mathbf{p} \equiv \mathbf{p}^{0} \\
{\left[-\nabla^{2}+V_{\text {eff }}(\mathbf{r})-E_{\nu}\right]\left|\dot{\varphi}^{0}\right\rangle=|\varphi\rangle}
\end{gathered}
$$

A partir do conjunto de funções de base introduzido na Eq. B.32 pode-se, enfim, obter as matrizes Hamiltoniana $\mathcal{H}^{0}$ e de overlap $\mathbf{O}^{0}$, ambas na representação canônica, como se segue [232]:

$$
\begin{array}{r}
\mathcal{H}^{0}={ }^{\infty}\left\langle\chi^{0}\left|-\nabla^{2}+V_{\text {eff }}(\mathbf{r})\right| \chi^{0}\right\rangle^{\infty} \\
=\left(\mathbb{1}+\mathbf{o}^{0} \mathbf{h}^{0}\right)^{\dagger} \mathbf{h}^{0}+\left(\mathbb{1}+\mathbf{o}^{0} \mathbf{h}^{0}\right)^{\dagger} E_{\nu}\left(\mathbb{1}+\mathbf{o}^{0} \mathbf{h}^{0}\right)+\left(\mathbf{h}^{0}\right)^{\dagger} E_{\nu} \mathbf{p h}{ }^{0}
\end{array}
$$

e

$$
\mathbf{O}^{0}={ }^{\infty}\left\langle\chi^{0} \mid \chi^{0}\right\rangle^{\infty}=\left(\mathbb{1}+\mathbf{o}^{0} \mathbf{h}^{0}\right)^{\dagger}\left(\mathbb{1}+\mathbf{o}^{0} \mathbf{h}^{0}\right)+\left(\mathbf{h}^{0}\right)^{\dagger} \mathbf{p} \mathbf{h}^{0}
$$

nas quais as expansões em termos de $\mathbf{h}^{0}, \mathbf{p}$, e $\mathbf{o}^{0}$ são obtidas utilizando-se as Eqs. B.33 a B.42. Portanto, nota-se que as funções de base $\left|\chi^{0}\right\rangle^{\infty}$ serão ortogonais se $\mathbf{O}^{0} \sim \mathbb{1}$, isto é, somente se o produto $\mathbf{o}^{0} \mathbf{h}^{0}$ puder ser desprezado, haja vista que os elementos da matriz p são pequenos [131]. Observe também que $\mathcal{H}^{0}$ e $\mathbf{O}^{0}$ levam os índices que as identificam como sendo da base canônica. De posse destas matrizes Hamiltoniana e de overlap (Eqs. B.43 e B.44) pode-se escrever, então, a equação secular do método LMTO (problema de 
autovalores) como:

$$
\left(\mathcal{H}^{0}-E \mathbf{O}^{0}\right) \mathbf{u}^{0}=\mathbb{0} .
$$

Com a finalidade de se obter explicitamente as matrizes $\mathbf{h}^{0}$ e $\mathbf{o}^{0}$, torna-se necessário o casamento contínuo e diferenciável na borda das esferas, ou seja, na superfície definida por $r=s$. Estas duas matrizes são, como é possível observar na Eq. B.32, as que efetivamente definem a base canônica [138]. Assim, cada função radial envelope, $k_{R l}^{0}(r)$ (Eq. B.23) e $j_{R l}^{0}(r)$ (Eq. B.24) - definidas em termos do parâmetro de escala $a=s$ -, deve ser casada com uma combinação linear de $\varphi_{R l}(r)$ e $\dot{\varphi}_{R l}(r)$, haja vista que estas funções por construção estão associadas à região interna da esfera em R. A expressão usual de substituição para que o casamento (ou "matching"), em um dado ponto de interesse $r=s$, de uma função genérica e diferenciável $f(r=s)$ com a combinação linear de duas funções $a(r=s)$ e $b(r=s)$ previamente estabelecidas que satisfaça as condições de contorno é obtida com o auxílio de Wronskianos [137] (Eq. B.16):

$$
f(s) \rightarrow \frac{a(s) \mathcal{W}(f, b)-b(s) \mathcal{W}(f, a)}{\mathcal{W}(a, b)}
$$

Logo, para $a(r) \equiv \dot{\varphi}_{R l}^{0}(r)$ e $b(r) \equiv \varphi_{R l}(r)$ é possível determinar as seguintes relações para os elementos de $\mathbf{h}^{0}$ e $\mathbf{o}^{0}[269]$ :

$$
h_{R^{\prime} L^{\prime}, R L}^{0}=\left[-\frac{\mathcal{W}\left(k_{R l}^{0}, \varphi_{R l}\right)}{\mathcal{W}\left(k_{R l}^{0}, \dot{\varphi}_{R l}^{0}\right)}+\sqrt{\frac{2}{s}} \mathcal{W}\left(j_{R l}^{0}, \varphi_{R l}\right) S_{R^{\prime} L^{\prime}, R L}^{0} \mathcal{W}\left(j_{R l}^{0}, \varphi_{R l}\right) \sqrt{\frac{2}{s}}\right]
$$

e a matriz diagonal $\mathbf{o}^{0} \equiv\left(o_{R l}^{0}\right)$

$$
o_{R l}^{0}=-\frac{\mathcal{W}\left(j_{R l}^{0}, \dot{\varphi}_{R l}\right)}{\mathcal{W}\left(j_{R l}^{0}, \varphi_{R l}\right)}
$$

De posse dos coeficientes $\mathbf{o}^{0}$ e $\mathbf{h}^{01}$, a base canônica $\left|\chi^{0}\right\rangle^{\infty}=|\varphi\rangle\left(\mathbb{1}+\mathbf{o}^{0} \mathbf{h}^{0}\right)+|\dot{\varphi}\rangle \mathbf{h}^{0}$ está completamente determinada. Contudo, no formalismo LMTO-ASA é comum separar o coeficiente $\mathbf{h}^{0}$ em termos de parâmetros que dependem e outros que independem do potencial. Isto será útil ao se definir a base ortogonal, pois a Hamiltoniana resultante poderá ser descrita de forma conveniente. Assim, introduzem-se os denominados parâmetros de

${ }^{1} \mathrm{~A}$ fim de facilitar o entendimento em determinadas passagens, matrizes como $\mathbf{h}^{0}, \mathbf{o}^{0}, \mathbf{C}^{0}, \boldsymbol{\Delta}^{0}$, entre outras, são às vezes referidas como coeficientes ou parâmetros, embora não se deva esquecer do seu caráter matricial. 
potencial $\mathbf{C}^{0}$ e $\boldsymbol{\Delta}^{0^{1 / 2}}$ (matrizes diagonais) na equação para $\mathbf{h}^{0}$ (Eq. B.47) objetivando reescrevê-la da seguinte maneira:

$$
h_{R L, R^{\prime} L^{\prime}}^{0}=\left(C_{R l}^{0}-E_{\nu R l}\right) \delta_{R, R^{\prime}} \delta_{L, L^{\prime}}+\left(\Delta_{R l}^{0}\right)^{1 / 2} S_{R L, R^{\prime} L^{\prime}}^{0}\left(\Delta_{R l}^{0}\right)^{1 / 2},
$$

onde são definidos os elementos de matriz:

$$
C_{R l}^{0}=E_{\nu R l}-\frac{\mathcal{W}\left(k_{R l}, \varphi_{R l}\right)}{\mathcal{W}\left(k_{R l}, \dot{\varphi}_{R l}^{0}\right)}
$$

e

$$
\left(\Delta_{R l}^{0}\right)^{1 / 2}=\sqrt{\frac{2}{s}} \mathcal{W}\left(j_{R l}^{0}, \varphi_{R l}\right)
$$

Portanto, $\mathbf{h}^{0}$ agora é expresso em termos: $(i)$ da matriz $\mathbf{S}^{0}$ (Eq. B.20), que depende da estrutura do problema, isto é, somente das posições atômicas $\mathbf{R}$ e $\mathbf{R}^{\prime}$ (em unidades do parâmetro $a=s$ ), porém é totalmente independente dos potenciais nas esferas muffin-tin; (ii) dos parâmetros $\mathbf{C}^{0}$ e $\Delta^{0^{1 / 2}}$, associados às condições de contorno nas esferas por meio dos Wronskianos e, logo, sujeitos ao potencial.

Assim, a priori, o problema está solucionado: da solução da Eq. B.45 considerandose a base descrita pela Eq. B.32, deriva a estrutura eletrônica do material investigado. Contudo, o procedimento de espaço direto só será viável se a matriz Hamiltoniana for localizada e esta característica não é garantida ao se utilizar a representação canônica. Isto porque a matriz $\mathbf{S}^{0}$, relacionada à distância entre os sítios $\mathbf{R}$ e $\mathbf{R}^{\prime}$ do material (Eq. B.20), demonstra um decaimento muito lento com a distância, proporcional a $\frac{1}{r^{2 l+1}}$, que se estende por diversas camadas de vizinhos - em particular para o caso dos orbitais $s(l=0)$ e $p(l=$ 1). Logo, $\mathbf{S}^{0}$ não é localizada e $\mathcal{H}^{0}$ também não o será. Visando contornar esse entrave, pode-se passar a trabalhar, dentro do formalismo LMTO-ASA, com sua representação geral, caracterizada pelos números de blindagem; esta representação possibilitou procurar um conjunto de base mais apropriado ao tratamento no espaço real, de interesse para sistemas com quebra de simetria de inversão. Sendo assim, nas próximas sub-seções este procedimento será descrito com mais detalhes.

\section{B.1.5 Formalismo LMTO-ASA na base genérica}

Uma das vantagens do LMTO-ASA, como foi argumentado anteriormente, é o fato de o problema de autovalores (Eq. B.2) poder ser resolvido em termos de outras bases que não a canônica, para a qual o método foi originalmente desenvolvido. Logo, a obtenção 
de uma nova base requer a construção inicial de um novo conjunto de funções envelope. Estas funções são as que definem os orbitais de base, tornando-os contínuos no espaço determinando seu alcance. Assim, o novo conjunto é gerado, novamente, pela realização do processo de augmentation, caracterizado pela substituição da função envelope nas regiões em que ela diverge por combinações de funções regulares $\varphi(r)$ e $\dot{\varphi}(r)$, por sua vez definidas para uma energia $E=E_{\nu}$ prévia e adequadamente escolhida. Trata-se, portanto, de um procedimento análogo à construção da base canônica. Visando diferenciar os parâmetros apresentados nesta seção, será adotado o índice $G$ - relativo à base genérica.

Procuram-se funções envelope que apresentem maior liberdade. Assim, o caminho natural é tomar as soluções gerais $K_{R L}^{G}(\mathbf{r}) \equiv k_{R l}^{G}(r) Y_{L}(\hat{\mathbf{r}})$ da equação de Laplace na vizinhança de $\mathbf{R}$, que são proporcionais a $K_{R L}^{0}(\mathbf{r})$. Definindo o chamado parâmetro de mistura (ou de blindagem), $\mathbf{Q}^{G}$ (matriz diagonal), e lembrando que as expansões de cauda em torno das demais esferas nos sítios $\mathbf{R}^{\prime} \neq \mathbf{R}$ são dadas nesta representação por

$$
K_{R^{\prime} L^{\prime}}^{G}\left(\mathbf{r}^{\prime}\right)=-\sum_{R^{\prime} L^{\prime}} J_{R^{\prime} L^{\prime}}^{G}\left(\hat{\mathbf{r}}^{\prime}\right) S_{R^{\prime} L^{\prime}, R L}^{G}
$$

então a função envelope $\left(K_{R L}^{G}\right)^{\infty}$ em todo o espaço (externo às esferas) é dada, em semelhança ao formalismo canônico (Eq. B.22), por:

$$
\left(K_{R L}^{G}\right)^{\infty}(\mathbf{r})=K_{R L}^{G}(\mathbf{r})-\sum_{R^{\prime} L^{\prime}} J_{R^{\prime} L^{\prime}}^{G}\left(\hat{\mathbf{r}}^{\prime}\right) S_{R^{\prime} L^{\prime}, R L}^{G}
$$

A nova base é obtida misturando-se uma fração $Q_{R^{\prime} L^{\prime}, R^{\prime} L^{\prime}}^{G} \equiv Q_{R^{\prime} L^{\prime}}^{G}$ de soluções irregulares $K_{R^{\prime} L^{\prime}}^{0}(\mathbf{r})$ às soluções nas esferas em $\mathbf{R}^{\prime}$, ou seja:

$$
J_{R^{\prime} L^{\prime}}^{G}\left(\hat{\mathbf{r}}^{\prime}\right) \equiv j_{R^{\prime} L^{\prime}}^{G}\left(r^{\prime}\right) Y_{L^{\prime}}\left(\hat{\mathbf{r}}^{\prime}\right)=J_{R^{\prime} L^{\prime}}^{0}\left(\mathbf{r}^{\prime}\right)-K_{R^{\prime} L^{\prime}}^{0}\left(\mathbf{r}^{\prime}\right) Q_{R^{\prime} L^{\prime}}^{G}
$$

As Eqs. B.53 e B.54 podem também ser escritas na forma vetorial usual do método LMTO como se segue:

$$
\left|K^{G}\right\rangle^{\infty}=\left|K^{G}\right\rangle-\left|J^{G}\right\rangle \mathbf{S}^{G}
$$

e

$$
\left|J^{G}\right\rangle=\left|J^{0}\right\rangle-\left|K^{0}\right\rangle \mathbf{Q}^{G}
$$

Como antes, os vetores linha $\left|K^{G}\right\rangle^{\infty},\left|J^{G}\right\rangle, \ldots$, possuem componentes $\left|K_{R L}^{G}\right\rangle^{\infty},\left|J_{R L}^{G}\right\rangle$, e assim por diante, que se anulam fora de sua esfera localizada em $\mathbf{R}$. O parâmetro $\mathbf{Q}^{G}$ determina o grau de mistura das funções irregulares, definindo a base em questão. Por exemplo, no caso de $\mathbf{Q}^{G} \equiv \mathbb{O}$ a base canônica é restaurada. É conveniente lembrar também 
que os elementos de mesmo sítio, $\mathbf{R}=\mathbf{R}^{\prime}$ (on-site), da matriz de estrutura canônica $\mathbf{S}^{0}$ são definidos como nulos, conforme a expressão para a função envelope $\left|K_{R L}^{0}\right\rangle^{\infty}$ (Eq. B.22). Por outro lado, considerando a matriz $\mathbf{S}^{G}$, estes elementos não são mais necessariamente nulos.

Comparando a Eq. B.25 com as Eqs. B.55 e B.56, encontra-se a expressão para a matriz de estrutura genérica (ou blindada) $\mathbf{S}^{G}$ em termos da matriz de estrutura canônica $\mathbf{S}^{0}$, pois a Eq. B.56 estabelece uma conexão entre $\left|J^{G}\right\rangle$ e $\left|J^{0}\right\rangle$ :

$$
\mathbf{S}^{G}=\mathbf{S}^{0}\left(\mathbb{1}-\mathbf{Q}^{G} \mathbf{S}^{0}\right)^{-1}
$$

É importante ressaltar que $\mathbf{S}^{G}$ também está relacionada à estrutura do material (como sua própria nomenclatura sugere), embora não seja totalmente independente do potencial nas esferas muffin-tin. Assim, a partir da Eq. B.57, as funções $\left|\mathbf{K}^{G}\right\rangle^{\infty}$ e $\left|\mathbf{K}^{0}\right\rangle^{\infty}$ ficam consequentemente relacionadas por:

$$
\left|\mathbf{K}^{G}\right\rangle^{\infty}=\left|\mathbf{K}^{0}\right\rangle^{\infty}\left(\mathbb{1}-\mathbf{Q}^{G} \mathbf{S}^{0}\right)^{-1}
$$

De modo exatamente igual ao realizado para a base canônica, pode-se definir a base $\left|\chi^{G}\right\rangle^{\infty}$ como função dos novos coeficientes $\mathbf{h}^{G}$ e $\mathbf{o}^{G}$ na base genérica (ansatz):

$$
\left|\chi^{G}\right\rangle^{\infty}=|\varphi\rangle+\left|\dot{\varphi}^{G}\right\rangle \mathbf{h}^{G}
$$

e

$$
\left|\dot{\varphi}^{G}\right\rangle=|\dot{\varphi}\rangle+|\varphi\rangle \mathbf{o}^{G}
$$

que, em analogia a $\left|\chi^{0}\right\rangle^{\infty}$ (Eq. B.32), se reduz a:

$$
\left|\chi^{G}\right\rangle^{\infty}=|\varphi\rangle\left(\mathbb{1}+\mathbf{o}^{G} \mathbf{h}^{G}\right)+|\dot{\varphi}\rangle \mathbf{h}^{G}
$$

utilizando-se das mesmas propriedades apresentadas nas Eqs. B.33 a B.42, porém no âmbito da base genérica em questão.

Ainda de maneira semelhante às Eqs. B.47 e B.48, os elementos que compõem os coeficientes $\mathbf{h}^{G} \equiv\left(h_{R^{\prime} L^{\prime}, R L}^{G}\right)$ e $\mathbf{o}^{G} \equiv\left(o_{R l}^{G}\right)$ (matriz diagonal) são obtidos a partir do casamento contínuo e diferenciável no contorno das esferas de cada função radial

$$
k_{R l}^{G}(r)=\left|\frac{r}{s}\right|^{-l-1}
$$




$$
j_{R l}^{G}(r)=\frac{1}{2(2 l+1)}\left|\frac{r}{s}\right|^{l}
$$

como uma combinação linear de $\varphi_{R l}(r)$ e $\dot{\varphi}_{R l}(r)$, com auxílio dos Wronskianos (Eq. B.16):

$$
h_{R^{\prime} L^{\prime}, R L}^{G}=\left[-\frac{\mathcal{W}\left(k_{R l}^{G}, \varphi_{R l}\right)}{\mathcal{W}\left(k_{R l}^{G}, \dot{\varphi}_{R l}^{G}\right)}+\sqrt{\frac{2}{s}} \mathcal{W}\left(j_{R l}^{G}, \varphi_{R l}\right) S_{R^{\prime} L^{\prime}, R L}^{G} \mathcal{W}\left(j_{R l}^{G}, \varphi_{R l}\right) \sqrt{\frac{2}{s}}\right]
$$

$\mathrm{e}$

$$
o_{R l}^{G}=-\frac{\mathcal{W}\left(j_{R l}^{G}, \dot{\varphi}_{R l}\right)}{\mathcal{W}\left(j_{R l}^{G}, \varphi_{R l}\right)}=-\frac{\mathcal{W}\left(j_{R l}^{0}, \dot{\varphi}_{R l}\right)-\mathcal{W}\left(k_{R l}^{0}, \dot{\varphi}_{R l}\right) Q_{R l}^{G}}{\mathcal{W}\left(j_{R l}^{0}, \varphi_{R l}\right)-\mathcal{W}\left(k_{R l}^{0}, \varphi_{R l}\right) Q_{R l}^{G}}
$$

Vale a pena destacar que, na Eq. B.65 verifica-se a relação direta entre os parâmetros $\mathbf{o}^{G}$ e $\mathbf{Q}^{G}$, ou seja, ao se definir um deles, o outro está automaticamente determinado. Esta expressão é derivada a partir da Eq. B.56.

Por fim, obtido o conjunto de funções de base $\left|\chi^{G}\right\rangle^{\infty}$ em termos de $\mathbf{h}^{G}$ e $\mathbf{o}^{G}$, as matrizes Hamiltoniana $\mathcal{H}^{G} \equiv \infty\left\langle\chi^{G}\left|-\nabla^{2}+V_{\text {eff }}(\mathbf{r})\right| \chi^{G}\right\rangle^{\infty}$, e de overlap $\mathbf{O}^{G} \equiv{ }^{\infty}\left\langle\chi^{G} \mid \chi^{G}\right\rangle^{\infty}$, ambas na base genérica, podem ser também encontradas, a saber:

$$
\mathcal{H}^{G}=\left(\mathbb{1}+\mathbf{o}^{G} \mathbf{h}^{G}\right)^{\dagger} \mathbf{h}^{G}+\left(\mathbb{1}+\mathbf{o}^{G} \mathbf{h}^{G}\right)^{\dagger} E_{\nu}\left(\mathbb{1}+\mathbf{o}^{G} \mathbf{h}^{G}\right)+\left(\mathbf{h}^{G}\right)^{\dagger} E_{\nu} \mathbf{p h}^{G}
$$

e

$$
\mathbf{O}^{G}=\left(\mathbb{1}+\mathbf{o}^{G} \mathbf{h}^{G}\right)^{\dagger}\left(\mathbb{1}+\mathbf{o}^{G} \mathbf{h}^{G}\right)+\left(\mathbf{h}^{G}\right)^{\dagger} \mathbf{p} \mathbf{h}^{G}
$$

onde, novamente, $E_{\nu}$ é uma matriz diagonal com elementos $E_{\nu R l}$.

O procedimento desenvolvido nesta sub-seção resulta em expressões de $\left|\chi^{G}\right\rangle^{\infty}, \mathcal{H}^{G}$ e $\mathbf{O}^{G}$ no formalismo LMTO-ASA válidas para qualquer valor do parâmetro $\mathbf{Q}^{G}$ (ou de $\mathbf{o}^{G}$ ). Assim, resta prosseguir com a determinação de outras bases de interesse, geradas a partir da escolha de parâmetros de mistura convenientes: as bases tight-binding (localizada) e ortogonal (na qual se toma $\mathbf{o}^{G} \equiv \mathbb{O}$, e, portanto, reduz a matriz overlap $\mathbf{O}^{G}$ à unidade).

\section{B.1.6 Base tight-binding (TB)}

A ideia fundamental por trás da criação desta base traduz-se na possibilidade de determinar um valor de $\mathbf{Q}^{G}$ de forma que o conjunto $\left|\chi^{G}\right\rangle^{\infty}$ seja o mais localizado possível, isto é, de maneira que apenas as interações entre os vizinhos mais próximos sejam suficientes para uma boa descrição do sistema investigado. Em termos das quantidades definidas anteriormente, significa dizer que $\mathbf{S}^{G}$ deve se constituir de elementos $S_{R L, R^{\prime} L^{\prime}}^{G}$ de curto alcance. Isto porque uma matriz Hamiltoniana descrita em termos de bases mais 
localizadas (matrizes esparsas) torna viável o uso do Método de Recorrência (seção B.2), adequado para o tratamento de problemas no espaço direto. Ademais, cálculos realizados com bases menores são mais rápidos e eficientes de serem executados. Em vista das Eqs. B.57 e B.58, que definem a base genérica, verifica-se que a base será localizada se a matriz de estrutura $\mathbf{S}^{G}$ conectar unicamente os sítios adjacentes.

No caso de metais de transição, em geral, o interesse concentra-se exclusivamente nos orbitais $s, p$ e $d(l \leq 2)$, e, logo, faz-se necessário escolher $Q_{l}^{G}=0$ para $l>2$. Na literatura [131] existem valores tabelados para $\mathbf{Q}^{G}$ (obtidos empiricamente) e convenientemente independentes do tipo de estrutura considerada, de tal forma que a matriz $\mathbf{S}^{G}$ correspondente apresenta o caráter localizado pretendido. Uma importante propriedade desta matriz é que esses valores decrescem exponencialmente com a distância entre os sítios. Além disso, toma-se $\mathbf{Q}^{G}$ independente do sítio, e será determinado por três parâmetros $Q_{s}^{G}, Q_{p}^{G}$, e $Q_{d}^{G}$. Aqui e doravante, os parâmetros associados à representação TB serão denotados com o sobrescrito barra (isto é, considera-se $\mathbf{Q}^{G} \equiv \overline{\mathbf{Q}}$, que localiza a base). Assim, os valores de $\overline{\mathbf{Q}}$ que dão a $\overline{\mathbf{S}}$ o alcance mais curto possível, quando calculados para as redes cúbicas ( $f c c, b c c$, e cúbica simples), apresentam a conveniente propriedade de serem aproximadamente independentes do tipo de rede, e explicitamente são dados por [130, 131]:

$$
\left\{\begin{array}{l}
\bar{Q}_{s}=0,3845 \\
\bar{Q}_{p}=0,05303 \\
\bar{Q}_{d}=0,010714 \\
\bar{Q}_{l} \equiv 0, \text { se } l \geq 3
\end{array}\right.
$$

Com o auxílio da Eq. B.57 é possível verificar que, se $\overline{\mathbf{Q}}$ depender unicamente de valores constantes, então a matriz $\overline{\mathbf{S}}$ se sujeitará apenas à estrutura do material por intermédio de $\mathbf{S}^{0}$. Na base TB, a equação de autovalores

$$
(\overline{\mathcal{H}}-E \overline{\mathbf{O}}) \overline{\mathbf{u}}=\mathbb{O}
$$

é descrita em termos dos parâmetros $\overline{\mathbf{h}}$ e $\overline{\mathbf{o}}$, por sua vez determinados pelas Eqs. B.64 e B.65 para os valores $\bar{Q}_{l}$ definidos na Eq. B.68. Usando as Eqs. B.66 e B.67, na representação genérica, e substituindo $\mathbf{h}^{G}$ e $\mathbf{o}^{G}$, respectivamente, por $\overline{\mathbf{h}}$ e $\overline{\mathbf{o}}$, obtém-se as seguintes relações para a matriz Hamiltoniana $\overline{\mathcal{H}}$ e de overlap $\overline{\mathbf{O}}$ :

$$
\overline{\mathcal{H}}=(\mathbb{1}+\overline{\mathbf{o}} \overline{\mathbf{h}})^{\dagger} \overline{\mathbf{h}}+(\mathbb{1}+\overline{\mathbf{o}} \overline{\mathbf{h}})^{\dagger} E_{\nu}(\mathbb{1}+\overline{\mathbf{o}} \overline{\mathbf{h}})+(\overline{\mathbf{h}})^{\dagger} E_{\nu} \mathbf{p} \overline{\mathbf{h}}
$$


$\mathrm{Ou}$

$$
\overline{\mathbf{O}}=(\mathbb{1}+\overline{\mathbf{o}} \overline{\mathbf{h}})^{\dagger}(\mathbb{1}+\overline{\mathbf{o}} \overline{\mathbf{h}})+(\overline{\mathbf{h}})^{\dagger} \mathbf{p} \overline{\mathbf{h}}
$$

Analogamente aos desenvolvimentos dos formalismos anteriores, é possível também escrever os elementos que compõem as matrizes $\overline{\mathbf{h}}$ e $\overline{\mathbf{o}}$, tomando como ponto de partida a representação genérica. Porém, antes de prosseguirmos com a apresentação definitiva do conjunto de base do LMTO-ASA utilizado para a vertente deste método no espaço real, cabe aqui comentar um pouco sobre a base ortogonal.

\section{B.1.7 Base ortogonal}

Na presente sub-seção será apresentada a base ortogonal, construída com o objetivo de facilitar a solução do problema de autovalores (Eq. B.2), e que é particularmente interessante para os sistemas analisados neste trabalho. Assim, uma sugestão natural é anular o parâmetro $\mathbf{o}^{G}$ na Eq. B.67 $\left(\mathbf{o}^{G}=\mathbb{O}\right)$, o que reduz a matriz de overlap, simplesmente, à unidade $\left(\mathbf{O}^{G}=\mathbb{1}\right)$. Isto porque, como mencionado anteriormente, os elementos da matriz p são pequenos [131], e o termo $\left(\mathbf{h}^{G}\right)^{\dagger} \mathbf{p h} \mathbf{h}^{G}$ na Eq. B.67 pode ser desprezado. Com a finalidade de não carregar a notação, os parâmetros na base ortogonal serão denotados sem índice. Logo, a matriz Hamiltoniana $\mathcal{H}$ pode ser escrita nesta representação como

$$
\mathcal{H}=\mathbf{h}+E_{\nu}
$$

uma vez que, com $\mathbf{o}^{G} \equiv \mathbf{o}=\mathbb{O},\left(\mathbb{1}+\mathbf{o}^{G} \mathbf{h}^{G}\right) \equiv \mathbb{1}$ na Eq. B.66. Portanto, a equação de autovalores será, nesta base

$$
\mathcal{H} \mathbf{u}=E \mathbf{u} \Rightarrow\left(\mathbf{h}+E_{\nu}\right) \mathbf{u}=E \mathbf{u}
$$

e, isolando h na equação acima:

$$
\mathbf{h}=\left(E-E_{\nu}\right) \sim \mathbf{h}^{G},
$$

considerando o caso usual no qual $\mathbf{o}^{G}$ não é rigorosamente zero, mas constituído de elementos suficientemente pequenos. Dessa maneira, escrevendo a Hamiltoniana $\mathcal{H}$ em termos dos parâmetros de potencial na base ortogonal $\left(\mathbf{C}, \boldsymbol{\Delta}^{1 / 2}\right.$ e $\left.\mathbf{Q}\right)$, a seguinte expressão é obtida:

$$
\mathcal{H}=\mathbf{C}+\Delta^{1 / 2} \mathbf{S} \Delta^{1 / 2}
$$


onde os elementos $C_{R l}$ e $\Delta_{R l}^{1 / 2}$ das matrizes $\mathbf{C}$ e $\boldsymbol{\Delta}^{1 / 2}$ determinam, nesta ordem, a posição (em unidades de energia) do centro da banda $R l$, e sua largura. A Eq. B.75 revela a forma confortável da Hamiltoniana nesta base, dividindo o problema em duas partes: uma dependente exclusivamente da espécie atômica investigada através de $\dot{\varphi}, \varphi$, e $E_{\nu}$; e outra, dependente da estrutura. Explicitamente, $C_{R l}$ e $\Delta_{R l}^{1 / 2}$ são escritos como:

$$
C_{R l}=E_{\nu}-\frac{\mathcal{W}\left(k_{R l}, \varphi_{R l}\right)}{\mathcal{W}\left(k_{R l}, \dot{\varphi}_{R l}\right)}
$$

e

$$
\Delta^{1 / 2}=\sqrt{\frac{2}{s}} \mathcal{W}\left(j_{R l}, \varphi_{R l}\right) .
$$

Assim, a nova matriz de estrutura $\mathbf{S}$ ortogonal, partindo da Eq. B.57, é dada por:

$$
\mathbf{S}=\mathbf{S}^{0}\left(\mathbb{1}-\mathbf{Q S}^{0}\right)^{-1}
$$

na qual o parâmetro $\mathbf{Q}$ é obtido pela escolha de $\mathbf{o}^{G} \equiv \mathbb{0}$ na Eq. B.65. Seus elementos assumem os valores:

$$
Q_{R l}=\frac{\mathcal{W}\left(j_{R l}, \dot{\varphi}_{R l}\right)}{\mathcal{W}\left(k_{R l}, \dot{\varphi}_{R l}\right)}
$$

Nas Eqs. B.78 e B.79 acima, nota-se que $\mathbf{S}$, ao contrário de $\mathbf{S}^{0}$ e $\overline{\mathbf{S}}$, apresenta uma dependência com a estrutura do material, pois o parâmetro $\mathbf{Q}$ está sujeito às condições de contorno na esfera muffin-tin. Em outras palavras, com a simplificação dos coeficientes, as informações relativas ao material investigado são transferidas à $\mathbf{Q}$ e $\mathbf{S}$ como funções do potencial. Portanto, a Hamiltoniana da Eq. B.75 não pode ser dividida em uma parte dependente e outra independente do potencial, tal como realizado no caso da representação canônica.

A determinação da função de base $|\chi\rangle^{\infty}$ (via Eq. B.61) consiste em tomar $\mathbf{o}^{G}=\mathbb{0}$ e trocar o coeficiente $\mathbf{h}^{G}$ pela relação obtida na Eq. B.72, resultando em:

$$
|\chi\rangle^{\infty}=|\varphi\rangle+|\dot{\varphi}\rangle\left(\mathcal{H}-E_{\nu}\right)
$$

que pode ser reescrita da seguinte maneira:

$$
|\chi\rangle^{\infty}=|\varphi\rangle+|\dot{\varphi}\rangle\left(E-E_{\nu}\right)
$$

É interessante observar que, no LMTO-ASA, as funções de base na representação ortogonal são dadas por uma expansão em série de Taylor até primeira ordem (linear) em energia de ondas parciais $|\varphi(E, r)\rangle$, ao redor de uma dada energia $E_{\nu}$. 


\section{B.1.8 Representação ortogonal da matriz $\mathcal{H}$ em termos de parâmetros da base TB}

Na seção B.1.5 anterior, a função envelope genérica foi definida segundo o conjunto de Eqs. B.55 e B.56. Por outro lado, com o auxílio dos parâmetros de mistura, nas Seções B.1.6 e B.1.7 desenvolveu-se o formalismo LMTO-ASA em ambas as bases TB e ortogonal. Essas transformações são necessárias e vantajosas para o tratamento de problemas no espaço direto, pois o uso da base ortogonal em conjunto com a base TB possibilitaria, ao mesmo tempo, a simplificação do problema de autovalores, por reduzir a matriz de overlap à unidade, e a utilização do Método de Recorrência (seção B.2). Entretanto, partindo da representação genérica, não há uma escolha do parâmetro $\mathbf{Q}^{G}$ que produza, concomitantemente, uma base localizada e ortogonal - tornando necessária a adoção de determinadas aproximações. Assim, nesta seção, convém apresentar as aproximações que permitem a obtenção de uma Hamiltoniana ortogonal em termos dos parâmetros de potencial na representação TB.

Inicialmente, expressa-se a Hamiltoniana ortogonal $\left(\mathcal{H}=\mathbf{h}+E_{\nu}\right)$ em termos da base generalizada. Isto é alcançado comparando-se os termos em $|\dot{\varphi}\rangle$ nas bases $|\chi\rangle^{\infty}$ (Eq. B.80) e $\left|\chi^{G}\right\rangle^{\infty}$ (Eq. B.61), o que resulta na seguinte relação entre $\mathbf{h}$ e $\mathbf{h}^{G}$ :

$$
\mathbf{h}=\mathbf{h}^{G}\left(\mathbb{1}+\mathbf{o}^{G} \mathbf{h}^{G}\right)^{-1}
$$

Logo, por $\mathbf{h}^{G}$ e $\mathbf{o}^{G}$ caracterizarem-se como coeficientes genéricos, a Eq. B.82 deve ser válida para qualquer base dentro do formalismo LMTO-ASA. Portanto, a Hamiltoniana $\mathcal{H}$ na base ortogonal, em termos da base TB, pode ser escrita como:

$$
\mathcal{H}=\mathbf{h}+E_{\nu}=\overline{\mathbf{h}}(\mathbb{1}+\overline{\mathbf{o}} \overline{\mathbf{h}})^{-1}+E_{\nu}
$$

Uma primeira aproximação é considerar que $(\overline{\mathbf{o}} \overline{\mathbf{h}})$ apresenta elementos suficientemente pequenos, validando a expansão do termo $(\mathbb{1}+\overline{\mathbf{o}} \overline{\mathbf{h}})^{-1}$ em uma série de potências, conhecida como série de Neumann, e reescrever a Hamiltoniana da Eq. B.83 da seguinte maneira:

$$
\mathcal{H}=E_{\nu}+\overline{\mathbf{h}}(\mathbb{1}-\overline{\mathbf{o}} \overline{\mathbf{h}}+\overline{\mathbf{o}} \overline{\mathbf{h}} \overline{\mathbf{h}}+\ldots)
$$

ou

$$
\mathcal{H}=E_{\nu}+\overline{\mathbf{h}}+\overline{\mathbf{h}}(-\overline{\mathbf{o}} \overline{\mathbf{h}}+\overline{\mathbf{o}} \overline{\mathbf{h}} \overline{\mathbf{h}}+\ldots) .
$$

Usando o fato que $\overline{\mathbf{h}}=\left(E-E_{\nu}\right)($ Eq. B.74) e considerando que na maioria dos sistemas de 
interesse uma boa aproximação é atingida considerando-se os termos até primeira ordem em $\left(E-E_{\nu}\right)$, é possível reescrevê-la:

$$
\mathcal{H}^{(1)}=E_{\nu}+\overline{\mathbf{h}}
$$

Neste caso, a vantagem do método aproximativo é que a Hamiltoniana $\mathcal{H}^{(1)}$ fornece resultados monoeletrônicos corretos em energia até a primeira ordem de $\left(E-E_{\nu}\right)$. A inclusão de mais termos apenas aumenta a precisão à medida em que se afasta da energia $E_{\nu}$. Ou seja, usando-se $\mathcal{H}$ na forma determinada pela Eq. B.86 as regiões de validade do LMTO-ASA se restringem àquelas em torno de $E_{\nu}$.

Dentro do formalismo desenvolvido no espaço direto, a Hamiltoniana utilizada é aquela da representação ortogonal escrita em termos dos parâmetros na base TB, em que a Eq. B.86 é modificada para:

$$
\mathcal{H}=\overline{\mathbf{C}}+\bar{\Delta}^{1 / 2} \overline{\mathbf{S}} \bar{\Delta}^{1 / 2}
$$

sendo equivalente construir a matriz $\mathcal{H}$ cujos elementos são:

$$
\mathcal{H}_{R L, R^{\prime} L^{\prime}}=\bar{C}_{R l} \delta_{R, R^{\prime}} \delta_{L, L^{\prime}}+\bar{\Delta}_{R l}^{1 / 2} \bar{S}_{R L, R^{\prime} L^{\prime}} \bar{\Delta}_{R l}^{1 / 2}
$$

na qual os parâmetros de potencial TB são definidos como:

$$
\bar{C}_{R l}=E_{\nu}-\frac{\mathcal{W}\left(\bar{k}_{R l}, \varphi_{R l}\right)}{\mathcal{W}\left(\bar{k}_{R l}, \dot{\bar{\varphi}}_{R l}\right)}
$$

e

$$
\bar{\Delta}_{R l}^{1 / 2}=\sqrt{\frac{2}{s}} \mathcal{W}\left(\bar{j}_{R l}, \varphi_{R l}\right)
$$

Os elementos dos parâmetros de potencial, representados na base ortogonal $\left(C_{R l}, \Delta_{R l}\right.$, e $Q_{R l}$ ) estão diretamente relacionados à solução da equação tipo-Schrödinger em cada esfera centrada na posição $\mathbf{R}$, e são calculados nos códigos LMTO-ASA de espaço k usuais. Estes parâmetros se encontram tabelados na literatura para metais de transição e são o ponto de partida para cálculos parametrizados [131]. No RS-LMTO-ASA os parâmetros de potencial são usados na representação ortogonal, sendo necessário empregar uma expressão que relacione $C_{R l}, \Delta_{R l}$ e $Q_{R l}$ da representação ortogonal com os parâmetros $\bar{C}_{R l}, \bar{\Delta}_{R l}$ e $\bar{Q}_{R l}$ na base TB. Andersen [131] demonstrou que isto é alcançado utilizando a seguinte relação:

$$
\frac{\bar{\Delta}_{R l}^{1 / 2}}{\Delta_{R l}^{1 / 2}}=\left[1-\left(Q_{R l}-\bar{Q}_{R l}\right) \frac{C_{R l}-E_{\nu}}{\Delta_{R l}}\right]=\frac{\bar{C}_{R l}-E_{\nu}}{C_{R l}-E_{\nu}} .
$$


O cálculo da Hamiltoniana $\mathcal{H}^{(1)}$ de primeira ordem na base ortogonal, expressa em termos de parâmetros de potencial da base TB, pode ser separado em duas partes. Uma delas depende do potencial em cada esfera e consiste na obtenção dos parâmetros $\overline{\mathbf{C}}$ e $\overline{\boldsymbol{\Delta}}$ que serão usados na determinação de $\mathcal{H}^{(1)}$ (Eq. B.87). A outra parte depende da estrutura do material, e consiste no cálculo da matriz de estrutura $\overline{\mathbf{S}}$ que, como mencionado, está diretamente relacionada à distância entre os sítios, sendo responsável por estabelecer a conexão entre eles.

\section{B.2 O Método de Recorrência}

Na seção B.1 mostrou-se que, no formalismo LMTO-ASA, a matriz Hamiltoniana pode ser escrita na base ortogonal em termos de parâmetros TB. Nesta representação no espaço direto, $\mathcal{H}$ é uma matriz $9 N \times 9 N(18 N \times 18 N$, caso se leve em consideração o grau de liberdade de spin), onde $N$ simboliza o número de átomos do aglomerado em questão, e 9 o número total de orbitais (um $s$, três $p$ e cinco $d$ ) na base localizada. Devido à dimensão considerável desta matriz (quando $N$ é grande), torna-se inviável encontrar os autovalores da Eq. B.2 e calcular as densidades de estados em semelhança aos métodos tradicionais [131]. Estendido por Haydock [135], o Método de Recorrência apresentado nesta seção é bastante apropriado, pois permite reduzir o problema tridimensional - envolvendo uma enorme quantidade de constituintes (da ordem de $10^{3}$ ) - ao de uma cadeia linear finita que preserva as informações relevantes do sistema. De fato, o algoritmo que apresentaremos nesta seção foi proposto inicialmente por C. Lanczos [270] na década de 1950, mas seu uso tradicional durante muito tempo foi puramente numérico, isto é, como uma técnica exata de diagonalização de matrizes extensas para problemas de muitos corpos envolvendo sistemas pequenos [271]. Mais tarde, Haydock estendeu o método e desenvolveu aplicações para sistemas físicos com vários graus de liberdade - e, por isso, nos referiremos à esse algoritmo como Método de Haydock.

O procedimento de Haydock consiste em efetuar uma mudança de base que permite reescrever a matriz Hamiltoniana na representação tridiagonal (matriz de Jacobi). A partir disso, são facilmente calculados os elementos diagonais da função de Green $G_{00}(E) \equiv\left\langle u_{0}\left|(E \mathbb{1}-\mathcal{H})^{-1}\right| u_{0}\right\rangle$ do sistema. Uma vez escolhido o estado inicial $\left|u_{0}\right\rangle$, definem-se os estados seguintes $\left|u_{1}\right\rangle$ e $\left|u_{2}\right\rangle$ a partir da seguinte relação (Relação de Recorrência):

$$
\mathcal{H}\left|u_{n}\right\rangle=a_{n}\left|u_{n}\right\rangle+b_{n+1}\left|u_{n+1}\right\rangle+b_{n-1}\left|u_{n-1}\right\rangle,
$$

sendo $\mathcal{H}$ a Hamiltoniana e $\left\{a_{n}, b_{n}\right\}$ os coeficientes que descrevem a interação dos estados 
ortonormais $\left|u_{n}\right\rangle$ com $\left|u_{n+1}\right\rangle$ e $\left|u_{n-1}\right\rangle$. Note que esta equação é simétrica devido à componente $\left|u_{n+1}\right\rangle$ de $\mathcal{H}\left|u_{n}\right\rangle$ ser idêntica à $\left|u_{n}\right\rangle$ em $\mathcal{H}\left|u_{n+1}\right\rangle$. Isto é uma consequência direta do fato de a Hamitoniana ser uma matriz hermitiana. Partindo da Eq. B.92, para $n=0$ :

$$
\mathcal{H}\left|u_{0}\right\rangle={ }_{0} a\left|u_{0}\right\rangle+b_{1}\left|u_{1}\right\rangle
$$

Multiplicando a Eq. B.93 pela matriz adjunta $\left\langle u_{0}\right|$, e usando a propriedade de ortonormalidade entre os estados $\left|u_{n}\right\rangle$, tem-se que:

$$
a_{0}=\left\langle u_{0}|\mathcal{H}| u_{0}\right\rangle
$$

Obtido $a$, pode-se, logo, prosseguir para o cálculo do coeficiente $b_{1}$ :

$$
b_{1}\left|u_{1}\right\rangle=\left(\mathcal{H}-a_{0}\right)\left|u_{0}\right\rangle
$$

Novamente, tomando o produto de $b_{1}\left|u_{1}\right\rangle$ por seu conjugado transposto $\left\langle u_{1}\right| b_{1}^{*} \equiv\left\langle u_{1}\right| b_{1}$ :

$$
\begin{aligned}
\left\langle u_{1}\left|b_{1}^{2}\right| u_{1}\right\rangle & =\left\langle u_{0}\left|\left(\mathcal{H}-a_{0}\right)^{\dagger}\left(\mathcal{H}-a_{0}\right)\right| u_{0}\right\rangle \\
b_{1}^{2} & =\left\langle u_{0}\left|(\mathcal{H}-a)^{\dagger}(\mathcal{H}-a)\right| u_{0}\right\rangle .
\end{aligned}
$$

Determinando $b_{1}$, pode-se também calcular o vetor $\left|u_{1}\right\rangle$ da seguinte maneira, baseada na Eq. B.95:

$$
\left|u_{1}\right\rangle=\frac{\left(\mathcal{H}-a_{0}\right)}{b_{1}}\left|u_{0}\right\rangle
$$

Com os valores de $a_{0}$ e $b_{1}$, além do vetor $\left|u_{1}\right\rangle$ determinados, os próximos parâmetros $a_{1}$ e $b_{2}$, e o estado seguinte $\left|u_{2}\right\rangle$ podem ser obtidos por meio de processo análogo. De posse destas quantidades, é, então, possível calcular $a_{2}, b_{3}$ e $\left|u_{3}\right\rangle$, e assim por diante. Portanto, sem perda de generalidade, para qualquer índice $0<n \leq N$ :

$$
\begin{gathered}
a_{n}=\left\langle u_{n}|\mathcal{H}| u_{n}\right\rangle, \\
b_{n+1}^{2}=\left[\left\langleu_{n}\left|\left(\mathcal{H}-a_{n}\right)^{\dagger}-\left\langle u_{n-1}\right| b_{n}^{*}\right]\left[\left(\mathcal{H}-a_{n}\right)\left|u_{n}\right\rangle-b_{n}\left|u_{n-1}\right\rangle\right],\right.\right. \\
\left|u_{n+1}\right\rangle=\frac{\left(\mathcal{H}-a_{n}\right)\left|u_{n}\right\rangle-b_{n}\left|u_{n-1}\right\rangle}{b_{n+1}} .
\end{gathered}
$$


Assim, vê-se que $\mathcal{H}$ é uma matriz tridiagonal na forma:

$$
\mathcal{H}=\left[\begin{array}{ccccc}
a_{0} & b_{1} & 0 & 0 & \ldots \\
b_{1} & a_{1} & b_{2} & 0 & \ldots \\
0 & b_{2} & a_{2} & b_{3} & \ldots \\
0 & 0 & b_{3} & a_{3} & \ldots \\
\vdots & \vdots & \vdots & \vdots & \ddots
\end{array}\right]
$$

A partir da construção da Hamiltoniana nesta forma simples, a densidade local de estados (LDOS: Local $\mathbf{D}$ ensity $\mathbf{O} f \mathbf{S}$ tates) projetada no orbital $\left|u_{0}\right\rangle$ de referência é calculada, pela fórmula de Lloyd [152], por [272]:

$$
n_{0}(E)=-\frac{1}{\pi} \mathcal{I} m\left[G_{00}(E)\right]=-\frac{1}{\pi} \mathcal{I} m\left[\left\langle u_{0}\left|(E \mathbb{1}-\mathcal{H})^{-1}\right| u_{0}\right\rangle\right],
$$

onde $G_{00}(E)$ é o primeiro elemento da diagonal principal da matriz $G_{0}(E)$.

Usando o formato da matriz $\mathcal{H}$ definido na Eq. B.101, a função de Green local correspondente ao estado $\left|u_{0}\right\rangle$, expressa em termos da base ortogonal $\left\{\left|u_{n}\right\rangle\right\}$, é:

$$
\begin{gathered}
\multicolumn{5}{c}{G_{0}(E)=(E \mathbb{1}-\mathcal{H})^{-1}=} \\
{\left[\begin{array}{ccccc}
\left(E-a_{0}\right) & -b_{1} & 0 & 0 & \ldots \\
-b_{1} & \left(E-a_{1}\right) & -b_{2} & 0 & \ldots \\
0 & -b_{2} & \left(E-a_{2}\right) & -b_{3} & \ldots \\
0 & 0 & -b_{3} & \left(E-a_{3}\right) & \ldots \\
\vdots & \vdots & \vdots & \vdots & \ddots
\end{array}\right] .}
\end{gathered}
$$

É conhecido que o elemento de $A_{i j}^{-1}$ da inversa de uma matriz genérica $A=\left(A_{i j}\right)$, por construção, é dada pela razão entre seu cofator ${ }^{1}$ e o determinante da matriz em questão:

$$
A_{i j}^{-1}=\frac{\operatorname{cof}(A)_{i j}}{\operatorname{det}(A)}
$$

Assim, por exemplo, o elemento $A_{11}^{-1}$ da matriz inversa é obtido pela a razão entre o cofator $\operatorname{cof}(A)_{11}$ de $(E \mathbb{1}-\mathcal{H})$ e o seu respectivo determinante. Logo, se $D_{n, n}$ é a determinante da matriz $(E \mathbb{1}-\mathcal{H})$ com as $n$ primeiras linhas e colunas suprimidas, tem-se por extensão

\footnotetext{
${ }^{1} \mathrm{O}$ cofator $\operatorname{cof}(A)_{i j}$ pode ser definido como o determinante da matriz cujas linha $i$ e coluna $j$ suprimidas da matriz $A$ original. A este resultado toma-se o produto por $(-1)^{i+j}$, que estabelece o sinal de $\operatorname{cof}(A)_{i j}$.
} 
que:

$$
G_{00}(E)=\frac{D_{1,1}}{D_{0,0}} \equiv \frac{D_{1,1}}{\operatorname{det}(E \mathbb{1}-\mathcal{H})}
$$

Se a expansão do determinante de uma matriz $A$ quadrada, de dimensão $n \times n$, em termos dos seus cofatores (expansão de Laplace), é expressa por

$$
\operatorname{det}(A)_{n \times n}=\sum_{i=1}^{n}(-1)^{i+1} A_{i 1} D_{i, 1}
$$

então:

$$
\begin{array}{r}
D_{0,0}=(-1)^{1+1}\left(E-a_{0}\right) D_{1,1}+(-1)^{2+1}\left(-b_{1}\right) D_{2,1}= \\
\left(E-a_{0}\right) D_{1,1}+b_{1} D_{2,1}
\end{array}
$$

e $D_{2,1}=(-1)^{1+1}\left(-b_{1}\right) D_{2,2}=-b_{1} D_{2,2}$. Assim, pode-se escrever o seguinte:

$$
D_{0,0}=\left(E-a_{0}\right) D_{1,1}-b_{1}^{2} D_{2,2}
$$

Mas, pelas definições da função de Green (Eqs. B.103 e B.105):

$$
G_{00}(E)=\frac{D_{1,1}}{D_{0,0}}=\frac{1}{\frac{D_{0,0}}{D_{1,1}}}=\frac{1}{\left(E-a_{0}\right)-b_{1}^{2} \frac{D_{2,2}}{D_{1,1}}} .
$$

Por outro lado, é possível expandir $D_{1,1}$ como:

$$
D_{1,1}=\left(E-a_{1}\right)(-1)^{2} D_{2,2}-\left(-b_{2}\right)^{2} D_{3,3}
$$

ou, genericamente:

$$
D_{n, n}=\left(E-a_{n}\right) D_{n+1, n+1}-\left(-b_{n+1}\right)^{2} D_{n+2, n+2} .
$$

Desta forma, tomando a substituição para $n \rightarrow \infty$ da Eq. B.111 na Eq. B.109, verifica-se que a função $G_{00}(E)$ pode ser expressa pela seguinte fração continuada:

$$
G_{0}(E) \equiv \frac{1}{\left(E-a_{0}\right)-\frac{b_{1}^{2}}{\left(E-a_{1}\right)-\frac{b_{2}^{2}}{\left(E-a_{2}\right)-\frac{b_{3}^{2}}{\left(E-a_{3}\right)-\ldots}}}} .
$$

Se a matriz $\mathcal{H}$ for esparsa (tight-binding) é possível truncar $G_{00}(E)$ em um certo ponto, limitado pelo parâmetro $n=L L$. Este procedimento leva a um espectro discreto. Uma 
maneira de contornar este problema é utilizar um terminador para simular os termos desprezados e formar um espectro contínuo. O método empregado neste trabalho e apresentado a seguir foi sugerido por Beer e Pettifor [136], em 1984. Neste caso, a fração continuada infinita é substituída pela expressão:

$$
G_{00}(E) \rightarrow \frac{1}{\left(E-a_{0}\right)-\frac{b_{1}^{2}}{\left(E-a_{1}\right)-\frac{b_{2}^{2}}{\ddots \frac{b_{L L}^{2}}{\left(E-a_{L L-1}\right)-\frac{b_{L L}^{2}}{\left(E-a_{L L}\right)-t(E)}}}}}
$$

Na Eq. B.113, $t(E)$ é chamado de terminador da fração continuada e representa a contribuição dos termos para $n>L L$. Toma-se, então, $a_{n} \equiv a$ e $b_{n} \equiv b$ constantes para neste intervalo e, como a fração original é infinita, é possível escrever:

$$
t(E) \equiv \frac{b^{2}}{E-a-t(E)}
$$

Portanto, para encontrar $t(E)$ basta resolver a equação de segundo grau:

$$
[t(E)]^{2}-(E-a) t(E)+b^{2}=0
$$

cuja solução é

$$
t(E)=\frac{1}{2}[(E-a) \pm \sqrt{(E-a-2 b)(E-a+2 b)}]
$$

Assim, a Eq. B.116, quando substituída na Eq. B.113, gera um espectro contínuo para a LDOS dentro do intervalo $a-2 b<E<a+2 b$.

Este processo é repetido para todos os orbitais centrados no mesmo sítio e, finalmente, somam-se suas respectivas contribuições. Isto gera uma densidade de estados total para este sítio. No caso da presente investigação, a fim de realizar o cálculo da densidade de estados nas esferas localizadas em $\mathbf{R}$ (com momento angular $L$ ), escolhe-se cada uma das funções de base $\left|u_{0}\right\rangle \equiv\left|\chi_{R L}^{0}\right\rangle$ como os estados iniciais. Logo, a densidade total de estados é obtida a partir da soma das LDOS relativas a cada um dos 9 orbitais considerados para o sistema. 


\section{Apêndice $\mathrm{C}$}

\section{Formalismo LKAG perturbado}

As expressões apresentadas na Seção 3.2.6, baseadas no formalismo de LiechtensteinKatsnelson-Antropov-Gubanov (LKAG) [77], são válidas apenas para os casos nos quais não há acoplamento spin-órbita, embora tenhamos considerado o quadro geral de uma configuração não-colinear de spins. Assim, uma ideia para levar em conta os efeitos do acoplamento spin-órbita é adicioná-lo ao formalismo como uma perturbação ao sistema [156-158]; com esse intuito, adotemos como notação o símbolo / para denotar os novos operadores e escalares perturbados. A partir deste ponto, nossa tarefa é tentar encontrar expressões para $\delta \mathbf{p}^{\prime}$ e $\tau^{\prime}$, a fim de inseri-las na Eq. 3.40 visando obter a nova variação da energia $\delta E_{i j}^{\prime}$ nessas condições. É possível demonstrar que a função de Green $\mathbf{G}_{i j}^{\prime}$ é dada por

$$
\mathrm{G}_{i j}^{\prime}=G_{i j}^{0} \mathbb{1}+\xi \sum_{k} G_{i k}^{0} \mathcal{H}_{k k}^{\mathrm{SO}} G_{k j}^{0}+\xi^{2} \sum_{k, l} G_{i k}^{0} \mathcal{H}_{k k}^{\mathrm{SO}} G_{k l}^{0} \mathcal{H}_{l l}^{\mathrm{SO}} G_{l j}^{0}+\cdots
$$

em que $\xi$ representa a constante de acoplamento spin-órbita, e a matriz $\mathcal{H}_{\nu \nu}^{\mathrm{SO}}$ é proporcional aos elementos de $\mathbf{L} \cdot \boldsymbol{\sigma}$, sendo $\mathbf{L}$ o operador de momento angular. Tomando a aproximação linear (de primeira ordem) em $\xi^{1}$, escrevemos então que

$$
\mathbf{G}_{i j}^{\prime} \sim \mathbf{G}_{i j}^{0}+\Delta \mathbf{G}_{i j}
$$

onde $\mathbf{G}_{i j}^{0}$ tem a estrutura de $G_{i j}^{0} \mathbb{1}$, enquanto $\Delta \mathbf{G}_{i j}$ é formado por um produto escalar do

\footnotetext{
${ }^{1}$ É interessante notar que o termo proporcional a $\xi^{2}$ é definido, à parte de outros fatores, como uma multiplicação de duas matrizes de Pauli com índices distintos $\nu$ e $\nu^{\prime}$. Entretanto, não consideraremos as correções de segunda ou maior ordem para a perturbação neste trabalho.
} 
tipo $\xi \boldsymbol{\Gamma}_{i j} \cdot \boldsymbol{\sigma}$. Logo, pela comparação com a Eq. C.1, direto conclui-se que as componentes do vetor $\boldsymbol{\Gamma}_{i j}$ podem ser obtidas segundo a expressão

$$
\Gamma_{i j}^{\mu}=\sum_{k} \mathbf{G}_{i k}^{0} L^{\mu} \mathbf{G}_{k j}^{0}
$$

onde o índice $\mu$ representa as coordenadas espaciais $x, y, z$. Usando o fato que $\Delta \mathbf{G}_{i j}=$ $\xi \boldsymbol{\Gamma}_{i j} \cdot \boldsymbol{\sigma}$, então o single site scattering operator na aproximação de primeira ordem em teoria de perturbação para o acoplamento spin-órbita pode ser obtido pela expansão

$$
\mathbf{t}_{i}^{\prime}=\mathbf{t}_{i}+\xi \mathbf{t}_{i}\left(\boldsymbol{\Gamma}_{i j} \cdot \boldsymbol{\sigma}\right) \mathbf{t}_{i}=\mathbf{t}_{i}\left[1+\xi\left(\boldsymbol{\Gamma}_{i j} \cdot \boldsymbol{\sigma}\right) \mathbf{t}_{i}\right] \Rightarrow\left(\mathbf{t}_{i}^{\prime}\right)^{-1} \sim \mathbf{t}_{i}^{-1}-\xi\left(\boldsymbol{\Gamma}_{i j} \cdot \boldsymbol{\sigma}\right)
$$

ou seja, por definição, e usando a separação de contribuições introduzida na Eq. (omitindo a dependência na energia $\varepsilon$ ), temos que

$$
\mathbf{p}_{i}^{\prime} \sim \mathbf{p}_{i}-\xi\left(\boldsymbol{\Gamma}_{i j} \cdot \boldsymbol{\sigma}\right)=p_{i}^{0}+\left(p_{i} \mathbf{e}_{i}-\xi \boldsymbol{\Gamma}_{i j}\right) \cdot \boldsymbol{\sigma}
$$

Isso implica na variação $\delta \mathbf{p}_{i}^{\prime} \sim p_{i} \delta \mathbf{e}_{i} \cdot \boldsymbol{\sigma}=\delta \mathbf{p}_{i}$. Assim, tendo obtido o valor da matriz $\mathbf{p}$ perturbada em termos da matriz $\mathbf{p}$ não-perturbada, resta encontrarmos uma expressão para $\tau^{\prime}$. Sabemos da TEM que o scattering path operator $\tau_{i j}$ é definido com base nos operadores $\mathbf{t}$ da seguinte forma

$$
\tau_{i j}=\mathbf{t}_{i} \delta_{i j}+\mathbf{t}_{j} \mathbf{G}_{i j}^{0}\left(1-\delta_{i j}\right) \mathbf{t}_{j}+\sum_{k \neq i} \mathbf{t}_{i} \mathbf{G}_{i k}^{0}\left(1-\delta_{i k}\right) \mathbf{t}_{k} \mathbf{G}_{k m}^{0}\left(1-\delta_{k m}\right) \mathbf{t}_{m}+\cdots
$$

o que acarreta diretamente na relação recursiva

$$
\tau_{i j}^{\prime}=\mathbf{t}_{i}^{\prime} \delta_{i j}+\sum_{k \neq i} \mathbf{t}_{i}^{\prime} \mathbf{G}_{i k}^{\prime} \tau_{k j}^{\prime} .
$$

Logo, é possível, pelas relações estabelecidas nas Eqs. C.1, C.4 C.6, e C.7, supor 
que o operador $\tau_{i j}^{\prime}$ é separável em duas principais contribuições, a saber:

$$
\tau_{i j}^{\prime}=\tau_{i j}+\Delta \tau_{i j}
$$

para o qual, após um longo exercício algébrico, encontra-se

$$
\Delta \tau_{i j}=\xi \mathbf{t}_{i}\left(\boldsymbol{\Gamma}_{i j} \cdot \boldsymbol{\sigma}\right) \mathbf{t}_{j} \delta_{i j}+\xi \mathbf{t}_{i}\left(\boldsymbol{\Gamma}_{i i} \cdot \boldsymbol{\sigma}\right) \mathbf{t}_{i} \mathbf{G}_{i j}^{0} \mathbf{t}_{j}+\xi \mathbf{t}_{i} \mathbf{G}_{i j}^{0} \mathbf{t}_{j}\left(\boldsymbol{\Gamma}_{j j} \cdot \boldsymbol{\sigma}\right) \mathbf{t}_{j}+\xi \mathbf{t}_{i}\left(\boldsymbol{\Gamma}_{i j} \cdot \boldsymbol{\sigma}\right) \mathbf{t}_{j}
$$

Além disso, demonstra-se que $\Delta \tau_{i j}$ tem a mesma estrutura que $\tau_{i j}$, isto é,

$$
\Delta \tau_{i j}=\Delta T_{i j}^{0} \mathbb{1}+\Delta \mathbf{T}_{i j} \cdot \boldsymbol{\sigma}
$$

cuja matriz transposta dos elementos possui a propriedade $\left(\Delta T_{i j}^{\alpha}\right)^{T}=-\Delta T_{j i}^{\alpha}$, onde $\alpha$ representa tanto o índice 0 , como as coordenadas espaciais $x, y, z$. Portanto, tal como a diferença de energia $\delta E_{i j}$ havia sido deduzida e apresentada na Eq. 3.40, o cálculo de $\delta E_{i j}^{\prime}$ resume-se em resolver a integral

$$
\begin{array}{r}
\delta E_{i j}^{\prime}=-\frac{1}{\pi} \int_{-\infty}^{\varepsilon_{F}} d \varepsilon \operatorname{Im} \operatorname{Tr}\left(\delta \mathbf{p}_{i}^{\prime} \tau_{i j}^{\prime} \delta \mathbf{p}_{j}^{\prime} \tau_{j i}^{\prime}\right) \sim-\frac{1}{\pi} \int_{-\infty}^{\varepsilon_{F}} d \varepsilon \operatorname{Im} \operatorname{Tr}\left(\delta \mathbf{p}_{i} \tau_{i j}^{\prime} \delta \mathbf{p}_{j} \tau_{j i}^{\prime}\right) \\
=-\frac{1}{\pi} \int_{-\infty}^{\varepsilon_{F}} d \varepsilon \operatorname{Im} \operatorname{Tr}\left[\delta \mathbf{p}_{i}\left(\tau_{i j}+\Delta \tau_{i j}\right) \delta \mathbf{p}_{j}\left(\tau_{j i}+\Delta \tau_{j i}\right)\right]
\end{array}
$$

na qual pretende-se substituir a relação da Eq. C.10, e o fato que $\delta \mathbf{p}_{i}=p_{i} \delta \mathbf{e}_{i} \cdot \boldsymbol{\sigma}$. Portanto, usando as propriedades do traço de matrizes, é simples demonstrar que $\operatorname{Tr}\left(p_{i} \Delta T_{i j}^{\alpha} p_{j} T_{j i}^{\beta}\right)=$ $-\operatorname{Tr}\left(p_{i} T_{i j}^{\beta} p_{j} \Delta T_{j i}^{\alpha}\right)$ e $\operatorname{Tr}\left(p_{i} T_{i j}^{\alpha} p_{j} \Delta T_{j i}^{\beta}\right)=-\operatorname{Tr}\left(p_{i} \Delta T_{i j}^{\beta} p_{j} T_{j i}^{\alpha}\right)(\operatorname{com}\{\alpha, \beta\}=\{0, x, y, z\}), \mathrm{a}$ partir das quais é possível deduzir as expressões finais apresentadas na Seção 3.2.6. 


\section{Apêndice D}

\section{Arquivo de entrada damping.in}

O desenvolvimento de um formalismo para o cálculo do damping por primeiros princípios mostrou que essa grandeza é inversamente dependente do momento magnético total $\left(m_{S}+m_{L}\right)$ do sítio de referência que se quer analisar (veja a Eq. 6.9). Assim, é necessário que haja uma leitura dos valores de $m_{S}$ e $m_{L}$ previamente calculados de forma autoconsistente (por primeiros princípios).

Além disso, como foi detalhado na Seção 6.1, o formalismo apresentado nesta tese é dependente de um broadening $\Lambda$ da função espectral, que é obtido naturalmente do terminador de Beer-Pettifor $\left(\Lambda_{R S}\right)$. Entretanto, para possibilitar a realização de alguns testes ou cálculos com valores de broadening existentes na literatura, deixou-se livre também para que o usuário possa alterar o valor de $\Lambda_{R S}$ e/ou incluir manualmente outro valor para corresponder à parte imaginária da energia.

Os valores de $m_{S}, m_{L}$, e do broadening são expressos no arquivo de input damping . in ${ }^{1}$. Esse arquivo organiza-se da seguinte forma:

\begin{tabular}{|rrr|}
\hline $\begin{array}{r}1.0 \mathrm{~d} 0 \\
0.0 \mathrm{~d} 0\end{array}$ & \\
& 3.0401915 & 0.0973288 \\
& 3.0053599 & 0.0918761 \\
$\ldots$ & & \\
& 2.4733827 & 0.1168890 \\
& 2.4733827 & 0.1168890 \\
$\ldots$ & & \\
\hline
\end{tabular}

\footnotetext{
${ }^{1}$ Se não houver arquivo de entrada damping. in a versão entende que não é necessário realizar cálculo ab-initio de damping.
} 
onde:

- na primeira linha, o 1.0 d0 representa que o usuário está utilizando 100\% do valor de $\Lambda_{R S}$ no cálculo. Caso queira utilizar apenas $90 \%$, por exemplo, a entrada deveria ser 0.9d0 (as porcentagens não são limitadas a 100\%);

- na segunda linha, o 0.0d0 representa que o usuário não está somando nenhum valor de broadening $\Lambda$, em eV, extra no cálculo. Portanto, ao colocar o valor 2.0d0, por exemplo, diz-se ao programa que são somados $2 \mathrm{eV}$ à parte imaginária da energia na função de Green (veja a Eq. 6.22);

- da terceira linha em diante, os valores como 3.04019150 .0973288 representam, nesta ordem, o $m_{S}$ e o $m_{L}$ do $i$-ésimo átomo. Esta parte do arquivo deve ter $N_{b u l k}+N_{\text {rec }}$ linhas, onde $N_{\text {bulk }}$ é o número de sítios do tipo bulk e $N_{\text {rec }}$ o número de átomos considerados no ciclo autoconsistente.

Trata-se, portanto, de um arquivo de entrada simples e que dá liberdade para que o usuário escolha a forma mais adequada do cálculo do damping, permitindo, por exemplo, a elaboração de estudos e testes como o apresentado na Fig. 6.11. 


\section{Apêndice E}

\section{Propriedades eletrônicas do Ir bulk}

Inicialmente, modelamos o bulk de Ir por um cluster contendo $~ 30.000$ átomos (hospedeiro puro). O Ir é um metal de transição $5 d$ que, no estado fundamental, cristaliza-se em uma estrutura cúbica de face centrada $(f c c)$, pertencente ao grupo 196 da tabela internacional de cristalografia. Seu parâmetro de rede experimental é de 3, $84 \AA$ [188], obtido à temperatura ambiente e pressão atmosférica. Esse valor coincide com o encontrado após otimização estrutural utilizando o método de pseudopotenciais do tipo Projector Augmented Wave (PAW) [178, 179] tal como implementado no código PWSCF do pacote Quantum ESPRESSO [180, 181], que resultou em uma variação desprezível de $\sim+0,8 \%$ no parâmetro de rede $\left(a_{\text {otm. }}=3,87 \AA\right)$ à temperatura $T=0 \mathrm{~K}$ e pressão nula. Assim, dada a pequena diferença encontrada, adotamos o valor experimental para o cálculo das propriedades eletrônicas do Ir bulk.

O cálculo do bulk teve como finalidade determinar sua energia de Fermi $\left(E_{F}\right)$ e parâmetros de potencial, necessários para a posterior convergência da superfície. Na estrutura cúbica de face centrada, os vetores primitivos $\mathbf{a}, \mathbf{b}, \mathbf{c}$ são definidos em termos dos versores cartesianos $\hat{\mathbf{x}}, \hat{\mathbf{y}}$ e $\hat{\mathbf{z}}$ como $\mathbf{a}=\frac{a}{2}(\hat{\mathbf{y}}+\hat{\mathbf{z}}), \mathbf{b}=\frac{a}{2}(\hat{\mathbf{x}}+\hat{\mathbf{z}})$, e $\mathbf{c}=\frac{a}{2}(\hat{\mathbf{x}}+\hat{\mathbf{y}})$, sendo $a$ o parâmetro de rede do material. O número de coordenação (isto é, de primeiros vizinhos) de cada átomo de Ir é 12 (com distância $a \frac{\sqrt{2}}{2}=2,72 \AA$ do átomo de referência), seguido por 6 segundos vizinhos (à uma distância $a=3,84 \AA$ ), 24 terceiros vizinhos (localizados a $a \sqrt{\frac{3}{2}}=4,70 \AA$ do sítio de referência), e assim por diante. Uma representação esquemática da célula convencional do Ir é apresentada na Figura E.1. Neste caso, e para todos os demais cálculos apresentados neste capítulo, usamos o parâmetro de corte $L L=22$ para o terminador de Beer-Pettifor. 


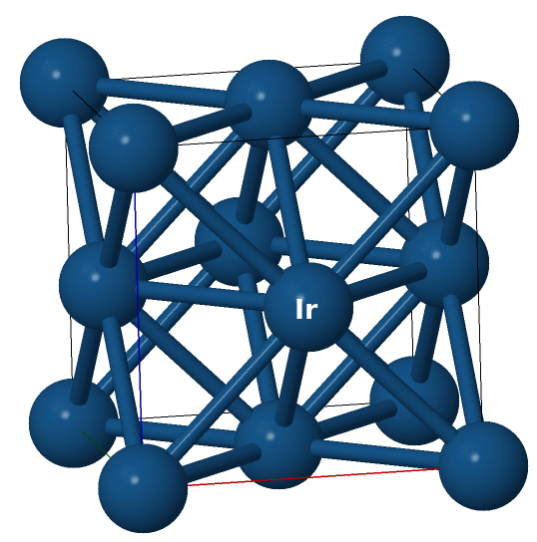

Figura E.1: Célula convencional da estrutura cristalina $(f c c)$ do Ir no estado fundamental.

A Figura E.2, abaixo, mostra a densidade local de estados (LDOS) total e por orbital calculada para o Ir bulk. Nota-se que a contribuição dominante para a densidade de estados no Ir, próximo ao nível de Fermi, é majoritariamente advinda da banda $d$, uma vez que esta concentra a maior ocupação dos estados de valência; por sua vez, as bandas com energias mais baixas são compostas sobretudo por estados $s$ e $p$. Observa-se, ainda, uma banda $d$ mais estreita do que a banda $s$, um comportamento típico e característico de metais de transição [188].

A análise da LDOS por orbital (Fig. E.2(b)) mostra um claro splitting dos orbitais $p$ e $d$ para uma dada direção de spin, por influência do elevado acoplamento spin-órbita do Ir. Esse splitting pode ser mais facilmente verificado quando o Ir bulk, para a mesma estrutura cristalina e parâmetro de rede, é calculado sem levar em conta o termo de spin-órbita na autoconsistência (Fig. E.3). Além disso, a Fig. E.2(b) mostra que todos os orbitais $d\left(d_{x y}, d_{y z}, d_{x z}, d_{x^{2}-y^{2}}\right.$, e $\left.d_{3 z^{2}-r^{2}}\right)$ estão igualmente presentes na região próxima ao nível de Fermi, formando de maneira equivalente, portanto, a superfície de Fermi do sistema. A simetria observada entre as chamadas bandas majoritárias $(\uparrow)$ e minoritárias $(\downarrow)$ de spin é responsável pelo caráter não-magnético do $\operatorname{Ir}\left(m_{S}=m_{L}=0\right)$. Por outro lado, a pequena LDOS no nível de Fermi caracteriza uma baixa susceptibilidade magnética para esse metal, diferentemente do que ocorre com a Pt, por exemplo [193, 273].

Na Figura E.3, nota-se que os orbitais $d$, quando não estão sujeitos à quebra de degenerescência provocada pelo acoplamento spin-órbita, agrupam-se em duas representações irredutíveis: o $e_{g}$, que corresponde aos orbitais $d_{x^{2}-y^{2}}$ e $d_{3 z^{2}-r^{2}}$ (bidegenerado), e o $t_{2 g}$, que compreende os orbitais $d_{x y}, d_{y z}$, e $d_{x z}$ (tridegenerado). Isso se deve ao splitting da banda $d$ causado pelo campo cristalino do material, que, por sua vez, tem origem na organização dos múltiplos íons no cristal fcc. 

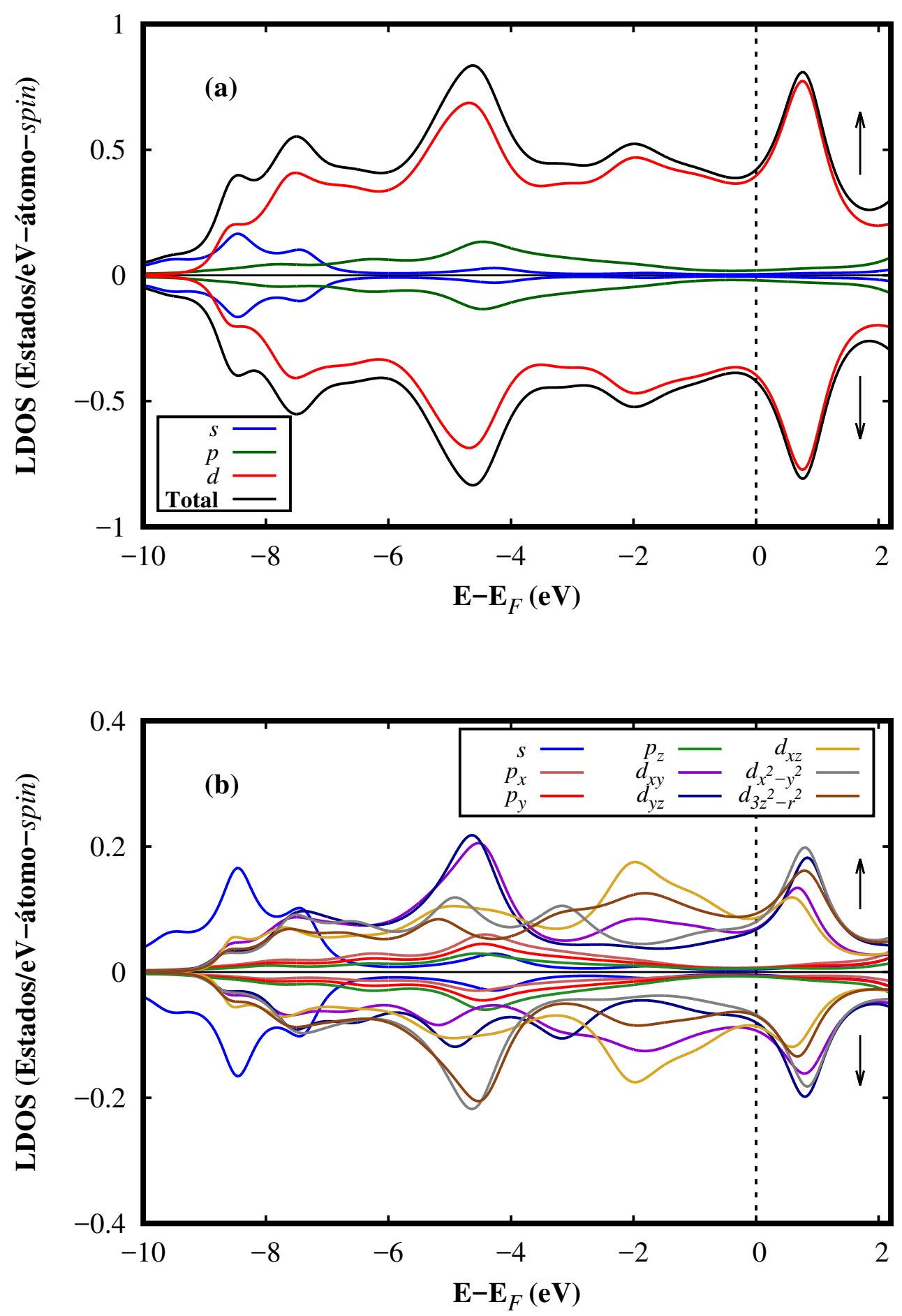

Figura E.2: Densidades de estados locais (LDOS): ( $a$ ) total e para cada estado $s, p$, e $d$; e (b) por orbital, calculadas para o Ir metálico (bulk) na estrutura cristalina de face centrada $(f c c)$. Em ambos considera-se o acoplamento spin-órbita na autoconsistência. As setas $\uparrow$ e $\downarrow$ indicam as ocupações por spin nas bandas majoritária e minoritária, respectivamente. $E_{F}$ é a energia de Fermi do sistema. 


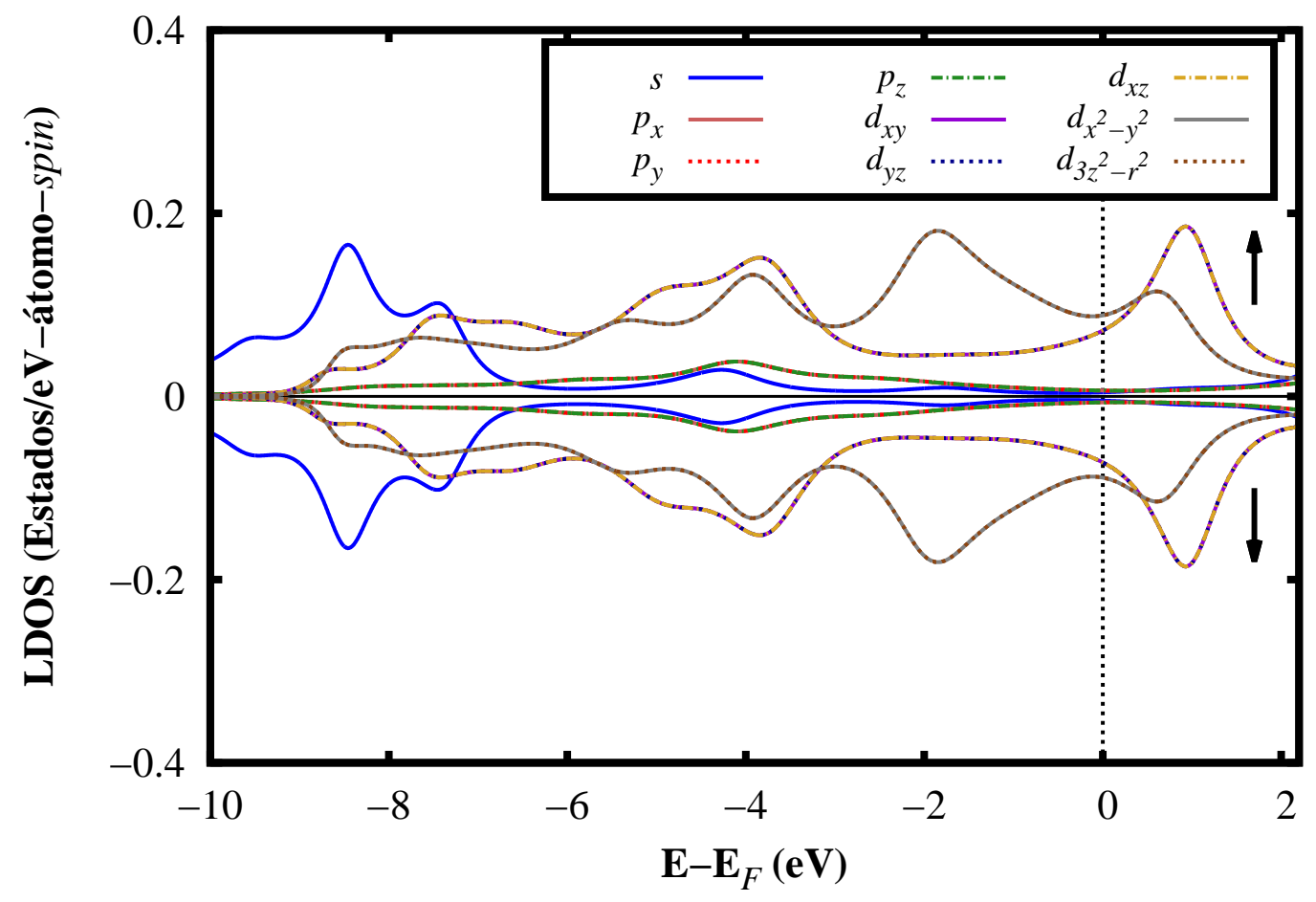

Figura E.3: LDOS por orbital, calculada para o Ir metálico (bulk) na estrutura cristalina de face centrada $(f c c)$, sem considerar o acomplamento spin-órbita na autoconsistência. As setas $\uparrow$ e $\downarrow$ indicam as ocupações por spin nas bandas majoritária e minoritária, respectivamente. $E_{F}$ é a energia de Fermi do sistema. 


\section{Apêndice F}

\section{UppASD com defeitos: implementação}

Embora o método RS-LMTO-ASA tenha sido empregado com sucesso diversas vezes na literatura para o cálculo de propriedades magnéticas de sistemas com quebra de simetria de inversão [142, 193, 274, 275], o pacote UppASD, para a realização de dinâmica de spins na aproximação atomística, é mais adequado para o tratamento de sistemas com condições periódicas de contorno. Em sua concepção original, o UppASD foi pensado para tratar materiais bulk [164], ou, no máximo, superfícies [276]. Entretanto, as operações de simetria são reduzidas à identidade quando consideramos sistemas contendo impurezas. Deve-se, portanto, utilizar alguns truques para realizar cálculos de dinâmica de spins confiáveis em sistemas com defeitos, partindo dos parâmetros obtidos via métodos de primeiros princípios; caso contrário, os arquivos de entrada seriam grandes demais para escrevê-los à mão.

Antes de prosseguir, porém, é importante enfatizar que o objetivo aqui é estudar impurezas embutidas em superfícies magnéticas. Ademais, neste Apêndice serão apresentadas as ideias que levaram aos resultados da Seção 5.4. O código-fonte para executá-las foi implementado na linguagem de programação Python3.

\section{F.1 Repetição de impurezas no espaço}

Dada a vocação do UppASD para o tratamento de sistemas com condições periódicas de contorno, um primeiro passo natural para posteriormente considerar um defeito iso- 
lado em uma superfície é a repetição periódica de impurezas no espaço. Este tipo de configuração apresenta um potencial interesse tecnológico, pois pode ser utilizado para a criação de caminhos e portas lógicas de skyrmions [200, 253] (veja a Fig. 6.12). Consideremos, portanto, uma ilha de Fe na camada de Co da multicamada Pd/Co/Ir(111), esquematicamente representada na Figura F.1. Para que não existam "buracos" no plano de superfície, é axiomático que a unidade de repetição tenha a estrutura de uma das redes de Bravais em duas dimensões. No presente caso, em se tratando de uma superfície $f c c(111)$, escolheu-se uma unidade de repetição hexagonal.

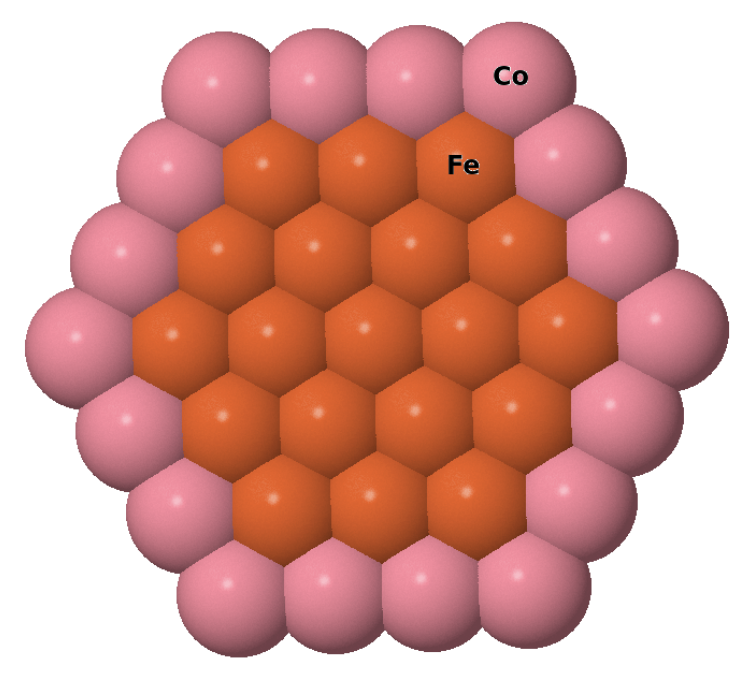

Figura F.1: Representação esquemática de uma ilha, de simetria hexagonal, contendo um defeito de Fe (19 átomos) localizado na camada de Co da multicamada Pd/Co/Ir(111). Esferas rosas e laranjas indicam os átomos de Co e Fe, respectivamente.

A fim de se obter bons resultados das simulações de dinâmica de spins, deve-se definir corretamente todos acoplamentos magnéticos $\left(\mathcal{J}_{i j}, \mathbf{D}_{i j}\right.$, e interações de ordem superior $^{1}$ ) entre os sítios no espaço-real. Como o RS-LMTO-ASA consegue calcular apenas os parâmetros $\mathcal{J}_{i j}$ e $\mathcal{D}_{i j}$ entre os sítios não-equivalentes considerados, tem-se ao final do processo as interações internas - no caso da Fig. F.1, entre os pares de átomos Fe-Fe, CoCo, e Fe-Co. Entretanto, visando formar um plano de superfície completo, essas unidades de repetição (ilhas) devem ser conectadas entre si. A Figura F.2 mostra um diagrama de conexões possíveis e distintas $J 1$, J2 e $J 3$ entre átomos da borda de uma ilha, com simetria quadrada (com objetivo didático). Supõe-se, então, que a conexão inter-unidades (i.e., a interação entre sítios $i$ e $j$ de ilhas distintas) tenha a mesma magnitude e direção ${ }^{2}$ de uma interação interna à unidade de repetição, e que compartilhe das mesmas espécies atômicas. Assim, por exemplo, assume-se que uma interação $\mathcal{J}_{\text {Co-Co }}$ entre duas ilhas dis-

\footnotetext{
${ }^{1}$ Ainda não implementadas.

${ }^{2}$ No caso do parâmetro $\mathbf{D}_{i j}$.
} 
tintas tem a mesma magnitude que uma interação $\mathcal{J}_{\text {Co-Co }}$ interna à ilha e com mesma distância entre os átomos de Co. Com isso, é possível conectar as ilhas e formar um plano de superfície coerente, contendo defeitos replicados pelo espaço.

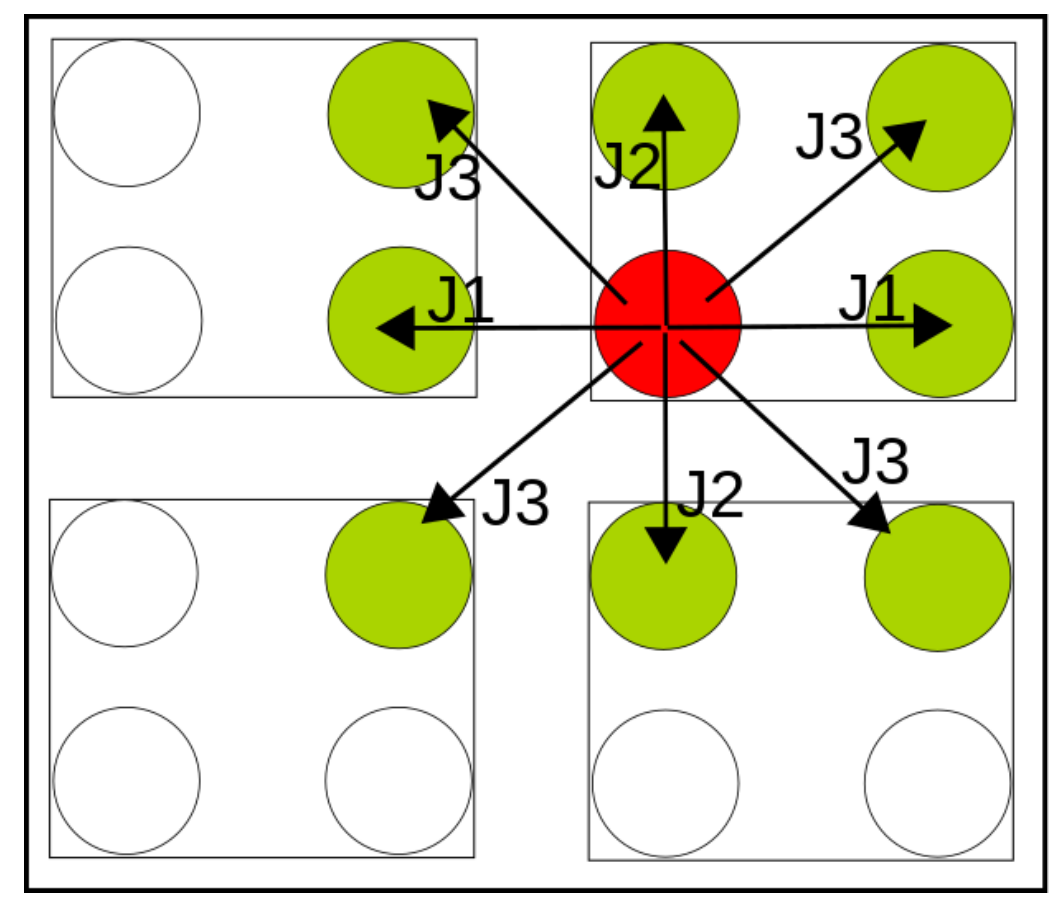

Figura F.2: Diagrama de conexões possíveis e não-equivalentes na simetria quadrada, de magnitudes $J 1, J 2$ e $J 3$, entre um sítio localizado na borda de uma ilha e sítios vizinhos localizados na própria ilha ou em ilhas distintas.

\section{F.2 Impureza isolada}

O próximo passo, naturalmente, é isolar a impureza na superfície. Assim como no caso da Seção F.1, defeitos isolados também apresentam um potencial interesse tecnológico, haja vista que podem servir como centros de pinning ou de repulsão de skyrmions, e, portanto, garantir o controle do seu movimento [277]. A Fig. 5.20, no texto principal, representa bem a configuração de interesse aqui.

Nessa situação, é razoável que, longe do defeito, as propriedades magnéticas dos átomos do substrato tendam àqueles da superfície limpa. Portanto, para estes podese utilizar as interações magnéticas previamente calculadas para o sistema puro. Entretanto, próximo à impureza (região cinza na Fig. 5.20), os acoplamentos magnéticos obtidos para o cluster não-equivalente devem ser considerados. Assim, a transição entre a região próxima ao defeito e a superfície limpa depende do tipo de cluster considerado nos cálculos ab-initio: (i) ou o cluster tem átomos de superfície suficientes para que ocorra 
essa transição (caso da Fig. 5.20), e a conexão com a superfície limpa é feita com os próprios parâmetros $\mathcal{J}_{i j}$ e $\mathbf{D}_{i j}$ encontrados via primeiros princípios; (ii) ou a conexão com a superfície limpa é feita a partir da definição de um raio máximo externo ao cluster (fora da região cinza na Fig. 5.20) em que parâmetros diferentes daqueles do sistema puro devem ser considerados - caso da Fig. F.1, já que claramente não há átomos de Co suficientes na borda para fazer a transição do defeito de Fe (esferas em laranja) com a superfície limpa de Co na multicamada $\mathrm{Pd} / \mathrm{Co} / \mathrm{Ir}(111)$. Na situação (ii), os parâmetros $\mathcal{J}_{i j}$ e $\mathbf{D}_{i j}$ fora do cluster (até o raio máximo) são procurados dentro daqueles obtidos via cálculos ab-initio para o cluster. Escolhe-se, então, aqueles que compartilham as mesmas espécies atômicas e a mesma direção da interação de interesse. 